UNITED STATES

DEPARTMENT OF THE INTERIOR

GEOLOGICAL SURVEY

SEDIMENT TRANSPORT BY IRRIGATION RETURN FLOWS IN FOUR SMALL DRAINS WITHIN THE DID-I 8 DRAINAGE OF THE SULPHUR CREEK BASIN, YAKIMA COUNTY, WASHINGTON, A PRIL I 979 TO OCTOBER I 98I

By Phillip R. Boucher

U.S. GEOLOGICAL SURVEY

Water-Resources Investigations Report 83-4167

Prepared in cooperation with the

STATE OF WASHINGTON DEPARTMENT OF ECOLOGY 
UNITED STATES DEPARTMENT OF THE INTERIOR

JAMES G. WATT, Secretary

GEOLOGICAL SURVEY

Dallas L. Peck, Director

For additional information write to:

District Chief

U.S. Geological Survey

1201 Pacific Avenue - Suite 600

Tacoma, Washington 98402-4384
Copies of this report can be purchased from:

Open-File Services Section Western Distribution Branch U.S. Geological Survey Box 25425, Federal Center Lakewood, Colorado 80225 (Telephone: (303) 234-5888) 


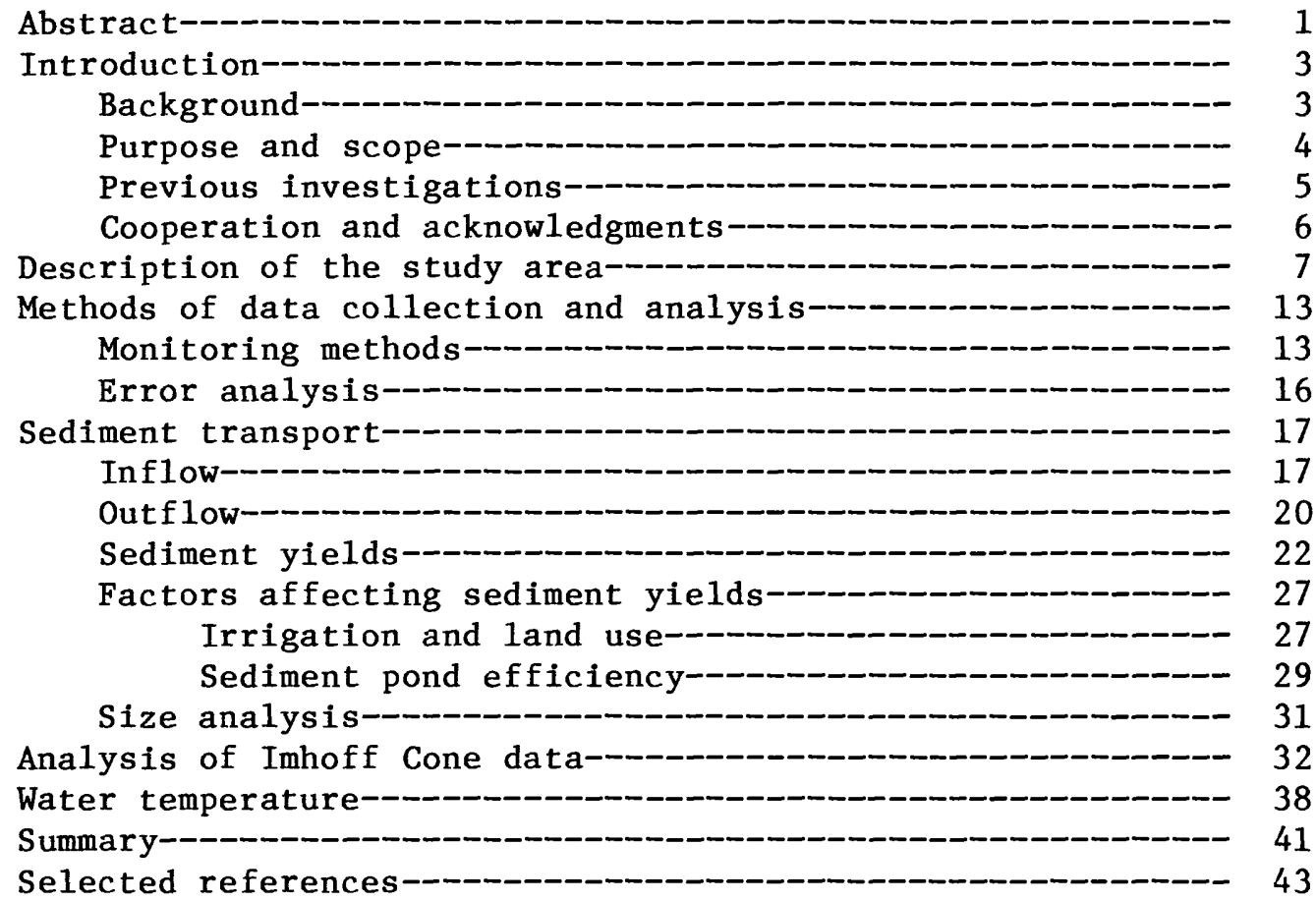

\section{ILLUSTRATIONS}

FIGURE 1. Map showing data-collection sites in DID-18 (Sulphur Creek basin)---

2. Hydrograph of monthly sediment discharge
Drains $61.0,60.7,59.6$, and 59.4 , near Sunnyside, Wash., for the period April 1979 to October 1981--

3. Sediment mass balances and least significant differences of Drains $61.0,60.7,59.6$, and 59.4 for the $1979,1980,1981$ irrigation seasons

4. Trap efficiency of sediment pond on Drain 60.7, on the basis of data obtained at sites 19-22---

5-8. Relation of sediment concentration to Imhoff Cone settleable solids during the period April 1979 to October 1981 at:

5. Site 1 (Drain 61.0 near Sunnyside)----- 34

6. Site 2 (Drain 60.7 near Sunnyside)-_-_- 35

7. Site 3 (Drain 59.6 below Drain 60.2, near Sunnyside)--_-_-_-_-_-_ 36

8. Site 4 (Drain 59.4 near Sunnyside)----- 37 
TABLE 1. Data-collection sites in DID-18-

2. Long-term average monthly precipitation and temperatures, and total precipitation and average monthly temperature at Sunnyside, Wash., during the period Apri1 1979-October 1981-

3. Crops grown and drainage area data for Drains $61.0,60.7$, 59.6 , and 59.4 , in the DID-18 drainage near Sunnyside, Wash., for the period 1979-81

4. Water outflows and inflows in four subbasins of the DID-18 Drain-

5. Sediment data for four subbasins of the DID-18 Drain-----

6. Sediment yields for four drains in the DID-18 Drain------

7. Median and mean water temperatures at five sites in the DID-18 basin of the Sulphur Creek basin for the 1979, 1980 , and 1981 irrigation seasons by harmonic analysis--

8a-d. Daily water discharge, suspended-sediment concentration, and suspended-sediment discharge for:

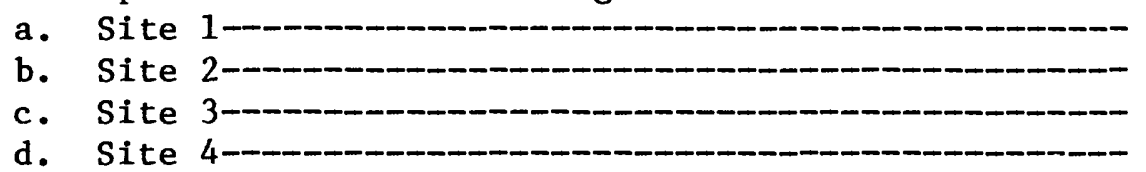

9a-d. Particle size analysis of sediment for:

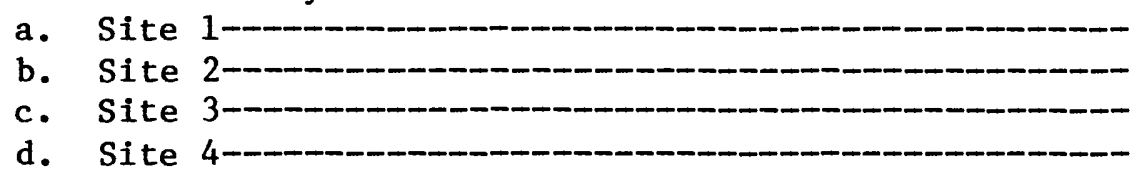

10a-d. Data from sediment diel studies and Imhoff Cone readings for:

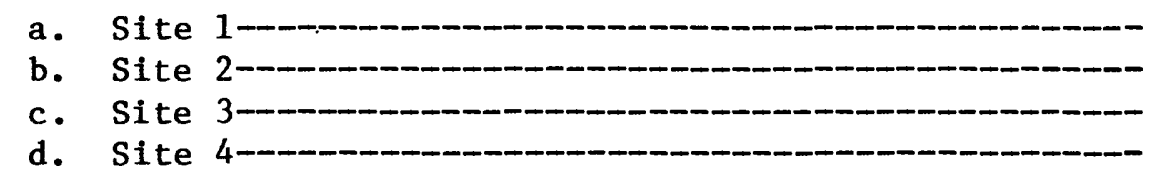

1la-d. Once-daily water temperatures for:

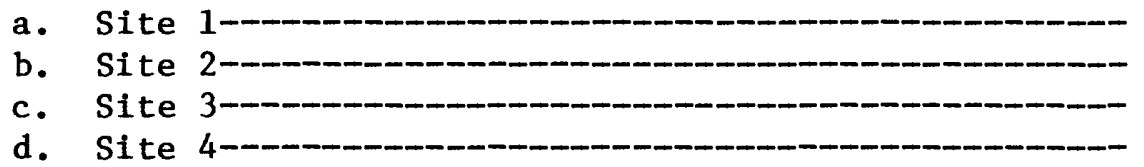

12. Analyses of miscellaneous suspended-sediment samples for sites in Drains $61.0,60.7,59.6$, and 59.4 near Sunnyside, Wash.-

13. Analysis of suspended-sediment samples taken at the
diversion on Roza Canal at mile 59.9 (site 7 ) near Sunnyside, Wash.

14. Miscellaneous sediment data for diversions (sites $5,6,8$, $9,10)$ from Roza Canal flowing into Drains $61.0,60.7$, 59.6 , and 59.4 near Sunnyside, Wash. 


\section{Mu1tip1y}

inches (in.)-

feet ( $f t)$

miles (mi)-_-___

square miles $\left(\mathrm{mi}^{2}\right)$ -

acres-_......

acre-feet (acre-ft)-_-

cubic feet per second ( $\mathrm{ft} 3 / \mathrm{s}) \ldots$

tons, short $(2,0001 \mathrm{~b})$

degree Fahrenheit
$\underline{B y}$

25.4
2.540
0.0254
0.3048
1.609
2.590
047.
233.
0.001233
0.02832

28.32

0.9072

0.264

To obtain

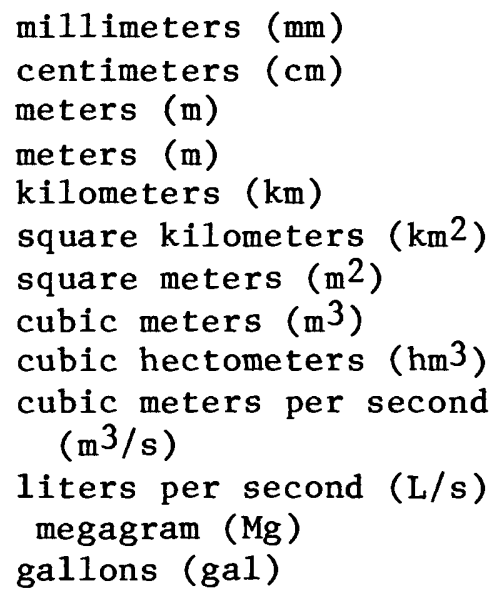

National Geodetic Vertical Datum of 1929 (NGVD of 1929): A geodetic datum derived from a general adjustment of the first-order level nets of both the United States and Canada, formerly called mean sea level. NGVD of 1929 is referred to as sea level in this report. 


\title{
SEDIMENT TRANSPORT BY IRRIGATION RETURN FLOWS IN \\ FOUR SMALL DRAINS WITHIN THE DID-18 DRAINAGE \\ OF THE SULPHUR CREEK BASIN, YAKIMA COUNTY, WASHINGTON, APRIL 1979 TO OCTOBER 1981
}

By Phillip R. Boucher

\begin{abstract}
Suspended sediment, water discharges, and water temperatures were monitored in four small drains in the DID-18 subbasin tributary to Sulphur Creek and to the Yakima River. The purpose was to provide a basis for evaluating the effectiveness of Best Management Practices in reducing sediment discharge from irrigated areas.

Sediment inflow for the 1979, 1980, and 1981 irrigation seasons was 298 tons, 119 tons, and 88 tons, respectively. The average sediment concentration of inflow water was $99 \mathrm{mg} / \mathrm{L}$ (milligrams per liter) in $1979,32 \mathrm{mg} / \mathrm{L}$ in 1980 , and $24 \mathrm{mg} / \mathrm{L}$ in 1981.

Sediment outflow minus inflow for the 1979, 1980, and 1981 irrigation seasons was 362 tons, 370 tons, 142 tons, and -19 tons in Drains 59.4, 59.6, 60.7, and 61.0, respectively; discharge-weighted mean sediment concentrations averaged $2,460 \mathrm{mg} / \mathrm{L}, 440 \mathrm{mg} / \mathrm{L}, 290 \mathrm{mg} / \mathrm{L}$, and $12 \mathrm{mg} / \mathrm{L}$, respectively. The sediment yield for the same drains for the 1979 to 1981 irrigation seasons averaged 1.5 tons per acre, 1.0 ton per acre, 0.6 ton per acre, and -0.16 ton per acre, respectively. The sediment yield from the four drains combined during the three irrigation seasons was 0.89 ton per acre. Drain 61.0 yields less sediment than it receives in irrigation water and acts as a diluter of sediment concentrations.

Sediment discharges could not be correlated with changes in Best Management Practices because Imhoff Cone readings taken in the interior of the basins showed no statistical differences between irrigation seasons. However, Drain 61.0, which acted as a sink for sediment, contained more sprinkler-irrigated land and had a smaller proportion of row crops than the other three drains, even though soils and slopes were similar. Sediment yield usually related best to acres of row crops.
\end{abstract}

The only major storm (February $16-21,1980$ ) produced 11 to 51 percent of the sediment discharged during the study.

A sediment pond in Drain 60.7 had an average trap efficiency of 70 percent for the three irrigation seasons. 
The regression relations between Imhoff Cone readings and suspended-sediment concentration for the outflow sites on Drains 60.7, 59.6, and 59.4 had corresponding correlation coefficients of $0.90,0.94$, and 0.90 , respectively, and standard errors of $1,070,2,410$, and $2,460 \mathrm{mg} / \mathrm{L}$, respectively. Use of the Imhoff Cone/suspended-sediment relationship to estimate sediment discharge generally gives poor results.

A comparison of median water temperatures at the Roza Canal diversion and at the outflow sites indicated that water in Drains 61.0 and 59.6 was about $1.5^{\circ} \mathrm{C}$ cooler, in Drain 60.7 about $0.5^{\circ} \mathrm{C}$ cooler, and in Drain 59.4 about $1.5^{\circ} \mathrm{C}$ warmer, than water from the canal. 


\section{INTRODUCTION}

\section{Background}

In 1972, Congress passed Public Law 92-500 (sec. 28), the Federal Water Pollution Control Act Amendments of 1972. The act set water-quality goals and established provisions for controlling or eliminating water pollution, wherever attainable, by 1985. The Federal Clean Water Act (Public Law 95-273) of 1977 amended the 1972 Act by declaring irrigation return flows to have a non-point source, and therefore not subject to the requirements of a discharge permit. Congress also prescribed goals and deadlines and the responsibilities of various government agencies. The U.S. Environmental Protection Agency (EPA) was designated as the Federal agency responsible for administering this law, but has reserved for the States the primary responsibility of administering the law. It is the States' responsibility to develop local solutions to local problems. Each State is required to submit a "Management Plan" as required in section 208, Public Law 95-217 to EPA for approval.

The Washington State Department of Ecology (WDOE) has prepared their "Irrigated Agriculture Water Quality Management Plan," which involves the concept of a performance standard to identify "problem farmers" or problem areas. Once a performance standard has been established, a quick and inexpensive method is needed to monitor on-farm effects of remedies for water quality, particularly changes in suspended-sediment concentration.

The standards would be based subjectively on what the 208 Water Quality County Committees set for irrigated agriculture. The committees then would decide the acceptable level of sediment concentration in irrigation return flows.

Traditional methods of rigorous sampling in the stream and of quality laboratory analysis are the most accurate measure of sediment yield. However, traditional means of sediment-data collection, analysis, and reporting involved too much time and cost to be acceptable under the proposed monitoring and enforcement (or advisory) situation. Because of these restrictions, the WDOE is using the Imhoff Cone to collect suspended-sediment data. These sediment data are required for setting the initial performance standard and for evaluating the effectiveness of Best Management Practices (BMP) in reducing suspended-sediment load.

During the same time frame, the Soil Conservation Service (SCS) and EPA selected seven "Model Implementation Plan" (MIP) projects to demonstrate the effectiveness of institutional and administrative implementation of BMP. The MIP projects were funded by the EPA. One MIP project was selected for parts of the Sulphur Creek and Granger Drain basins; it was the only irrigated area chosen out of the seven MIP projects, and the only one chosen in the western United States. 
Before setting the Imhoff Cone Standard, the 208 Water Quality County Committees requested that the WDOE collect data on individual farms throughout several irrigated areas in eastern Washington. Technical advice was provided by the WDOE multi-agency Irrigated Agriculture Technical Advisory Committee for Water Quality. Beginning with the meeting on October 1, 1974, the committee has addressed itself specifically to irrigated agriculture in the Yakima River basin. WDOE decided to test the sampling procedures using Imhoff Cone samples on a more detailed basin. Four small drainages in the DID-18 drainage of the Sulphur Creek basin were chosen for monitoring the results of implemented BMP's.

\section{Purpose and Scope}

The MIP monitoring study described in this report was coordinated by the WDOE Technical Advisory Committee on Irrigated Agriculture, which had coordinated some previous studies in the area. The MIP study included monitoring of irrigation return flows from individual farms by the WDOE. The U.S. Geological Survey's Water Resources Division, in cooperation with WDOE, was responsible for collecting water samples at inflow and outflow points and collecting streamflow data in order to evaluate sediment transport in four small subbasins in the Sulphur Creek basin for the period April 1979 to October 1981. The purpose of this study was to determine (1) the sediment mass balance, or sediment yields, and error analysis; (2) the probable factors affecting the difference in sediment yields; and (3) the relation between Imhoff Cone and standard suspended-sediment concentration at the four outflow points. This report describes the results of the study. 


\section{Previous Investigations}

Sulphur Creek basin, including the DID-18 Drain and part of the Roza Canal drainage, was studied by Boucher and Fretwell (1981) during the 1976 irrigation and 1976-77 nonirrigation seasons. The project was discontinued because of two major deficiencies: (1) farmer participation was only morerately successful; and (2) the necessary sampling network was too complex and expensive because of trans-basin flows.

During the 1974 irrigation season $\mathrm{CH}_{2} \mathrm{M}$ Hill (1975), a private consulting firm, conducted a study of irrigation return flows in the Yakima River that also included the Sulphur Creek basin.

Washington State University (WSU) conducted a program to monitor the quality of irrigation return flows at specific farm sites as part of the Sulphur Creek pilot project. The WSU program, under the direction of Dr. Larry King, began with the 1976 irrigation season. The data are on file at WSU's Department of Agricultural Engineering in Pullman, Wash. (written commun., 1982). Dr. King is studying the relationship of settleable solids obtained by the Imhoff Cone to suspended-sediment concentrations collected by standard methods; he is also studying soil types and their relationship to Imhoff Cone measurements.

Nelson (1979) studied suspended-sediment transport in irrigation return flows from several drains, including the Sulphur Creek Wasteway, in the Yakima River basin during the 1975 and 1976 irrigation seasons.

The U.S. Bureau of Reclamation (USBR) has also collected water-quality data in the Yakima River basin for many years, including data from various drains such as the Sulphur Creek Wasteway. These USBR data are on file at their project office in Yakima, Wash.

The soils in the study area have been described by the Soil Conservation Service in "Soil Surveys of Yakima County" (U.S. Soil Conservation Service, 1970). 


\section{Cooperation and Acknowledgments}

The project was done in cooperation with the WDOE whose personnel sampled irrigation return flows from fields and from monitoring sites with the Imhoff Cone, took standard calibration samples at the outflow monitoring sites, removed samples from automatic samplers and composited them into daily samples, and took Imhoff Cone samples at the monitoring sites. They also generally sampled water once daily at one inflow site, and provided assistance with the diel (24-hour period) studies.

Cliff Eckhart, MIP Project Manager, provided valuable assistance in obtaining various data related to BMP and in collecting data during the 1980-81 nonirrigation season.

The sediment concentration analyses were done by the U.S. Bureau of Reclamation in Euphrata, Wash., under the direction of Allan Hathrop.

Hank Vancik, manager of the Roza Irrigation District, provided space for a field laboratory and provided the inflow-water discharge data.

Thanks is given to Raymond E. Larson of Arnell Farms, Inc., and Cliff and Dale Van Belle of the Van Belle farm for permission to install automatic samplers and gaging stations on their property. 


\section{DESCRIPTION OF THE STUDY AREA}

The study area is located east of Wasteway No. 5, north of Van Belle Road, and west of Bethany Road (fig. 1). The sampling frequency and locations are listed in table 1 and locations are shown in figure 1 . Data were collected primarily from four small drains $(61.0,60.7,59.6$, and 59.4) located in the DID-18, which lies above the Sunnyside Canal in the Sulphur Creek drainage. A description of the Sulphur Creek basin is found in Boucher and Fretwell (1982) and $\mathrm{CH}_{2} \mathrm{M}$ Hill (1975).

The study area is divided by the Roza Canal into the nonirrigated portion, north of the canal, and the irrigated portion, south of the canal. Two of the study drains, 60.7 and 61.0, extend north of the canal. The nonirrigated drainage areas (north) and irrigated drainage areas (south) are as follows:

\begin{tabular}{cccc}
\hline Drain & $\begin{array}{c}\text { North of } \\
\text { Roza Canal } \\
(\text { nonirrigated) } \\
\left(\mathrm{mi}^{2}\right)\end{array}$ & $\begin{array}{c}\text { South of } \\
\text { Roza Canal } \\
\text { (irrigated) } \\
\left(\mathrm{mi}^{2}\right)\end{array}$ & $\begin{array}{c}\text { South of Roza } \\
\text { Canal and north } \\
\text { of SLI Road } \\
\text { (irrigated) } \\
\left(\mathrm{mi}^{2}\right)\end{array}$ \\
\hline 61.0 & 2.98 & & 0.12 \\
60.7 & .51 & 0.24 & .14 \\
59.6 & 0 & .42 & .45 \\
59.4 & 0 & .69 & .20 \\
\hline
\end{tabular}

The irrigated drainage in the study area south of Roza Canal is divided by the east-west-trending SLI Road, which crosses all four drains about midway in their north-south course and acts as a barrier. Flows north and south of SLI Road are connected by eight culverts under the road. The drainage areas for these sections are shown in the table above.

Streams in the study area south of the Roza Canal are perennial; prior to development of irrigation they were ephemeral. Even now, the extreme upper reaches of all drains in the irrigated portion and the nonirrigated upper reaches of Drains 61.0 and 60.7 are ephemeral. Seepage from the canals, laterals, and irrigation of farmed lands has augmented the irrigation return flows. The present drain courses reflect historical changes to allow for better use of the land. The drainages in some of the upper reaches have very flat banks even where they are being cultivated. Places in the lower reaches are deeply incised, but have fairly stable banks because of bushes and other plants growing along them. Aquatic growth is heavy in the main channels that remain undisturbed. Most channels are narrow, only 4-5 feet wide. 


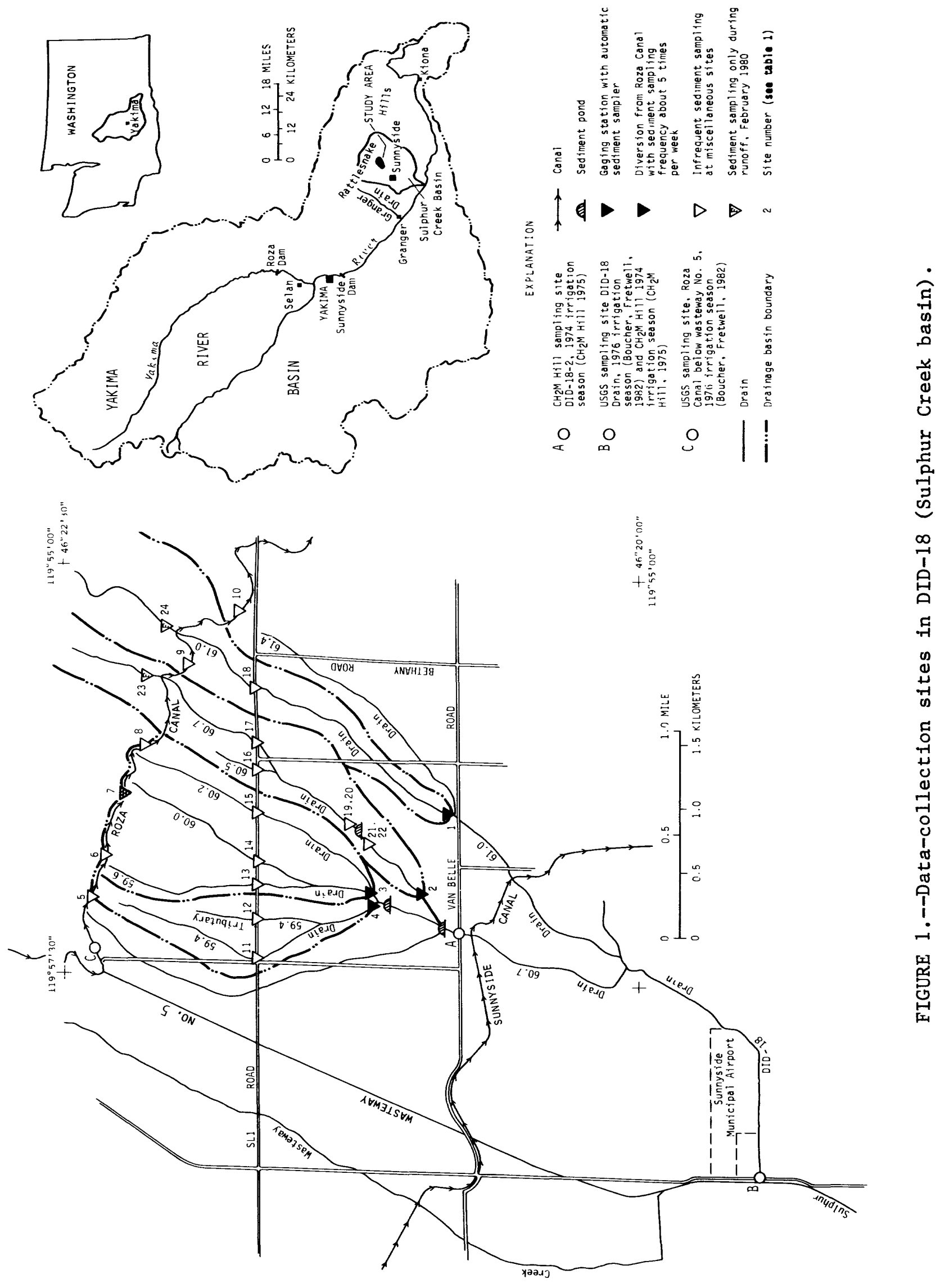




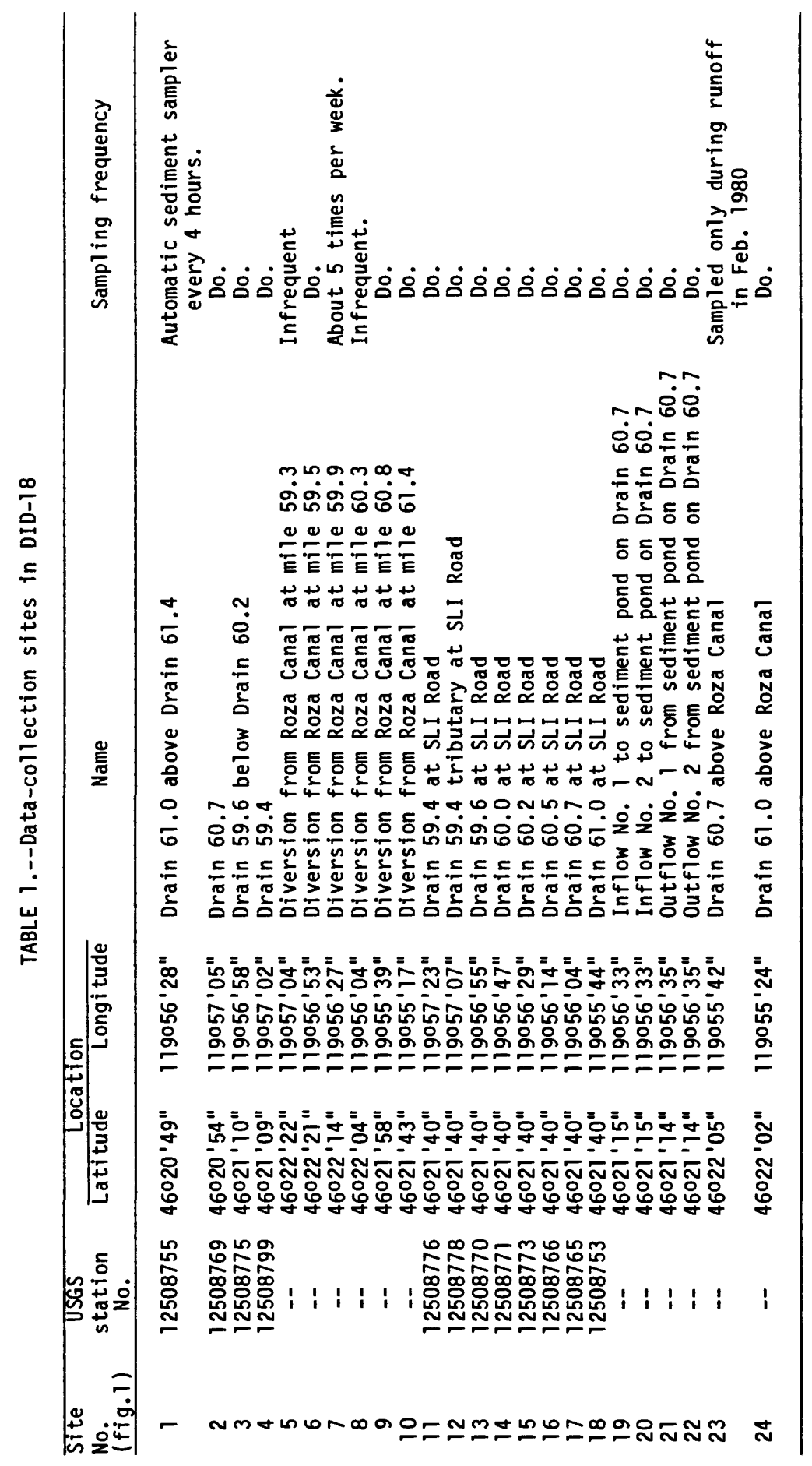


Slopes in all four drain basins range from 2 to 4 percent with an average of 3 percent. Slopes are steeper north of Roza Canal than those below the canal for Drains 60.7 and 62.0. The topography south of Roza Canal is similar in the four basins, with slightly less slope for Drain 59.4 .

The topographic relief of Drains 59.4 and 59.6 is about 240 feet, with 170 feet of relief north of SLI Road. The relief of Drains 60.7 and 61.0 below Roza Canal is about 225 and 240 feet, with 90 and 75 feet of relief between SLI Road and Roza Canal. For Drain 60.7 and 61.0 in the nonirrigated portion above Roza Canal the relief is about 470 and 1,360 feet. The upper reaches of Drain 61.0 reach an altitude of 2,460 feet above sea level. Altitudes at the lower end of the drains range from 870 to 855 feet. The altitude along the Roza Canal is about 1,110 feet.

The primary soils in the four basins are Burke loam and Sagemoor loam (very fine sandy loams) north of SLI Road and Sagemoor loam south of SLI Road (U.S. Soil Conservation Service, 1970). Minor soil types include the Esquatzel and Ritzville series. Because of the large percentage of very fine sand and silt and the moderate to steep slopes, erosion in the four drains is moderate to high. Drain 61.0 had slightly greater potential for erosion than the other three drains on the basis of soil types and slopes, and Drain 59.4 had slightly less potential. Drains 59.6 and 60.7 were about equal.

The climate is arid. Annual rainfall averages 6.8 inches at Sunnyside and ranges up to 15 inches in the Rattlesnake Hills to the northeast. Climatic data are from the U.S. National Oceanic and Atmospheric Administration (1979, 1980, and 1981). Most precipitation falls in the form of rain, but in the winter snow is common. The long-ter $m$ precipitation and temperature averages and precipitation and monthly temperature averages during the study period for the weather station at Sunnyside are listed in table 2. Precipitation was about 116 percent of nor mal, and temperatures averaged about $10 \mathrm{~F}\left(0.6^{\circ} \mathrm{C}\right)$ warmer than the long-term average.

Land use in the study area is irrigated agricultural. The percentages of crops grown in each subbasin are listed in table 3. Areas used for asparagus, beans, corn, mint, and small grains are tilled each year. The area in these crops was about 19 percent for Drain 61.0, 43 percent for Drain 60.7, 61 percent for Drain 59.6, and 89 percent for Drain 59.4. About 22 percent of Drain 61.0, 13 percent for Drain 60.7, 16 percent of Drain 59.6, and 7 percent of Drain 59.4 was in fallow. 


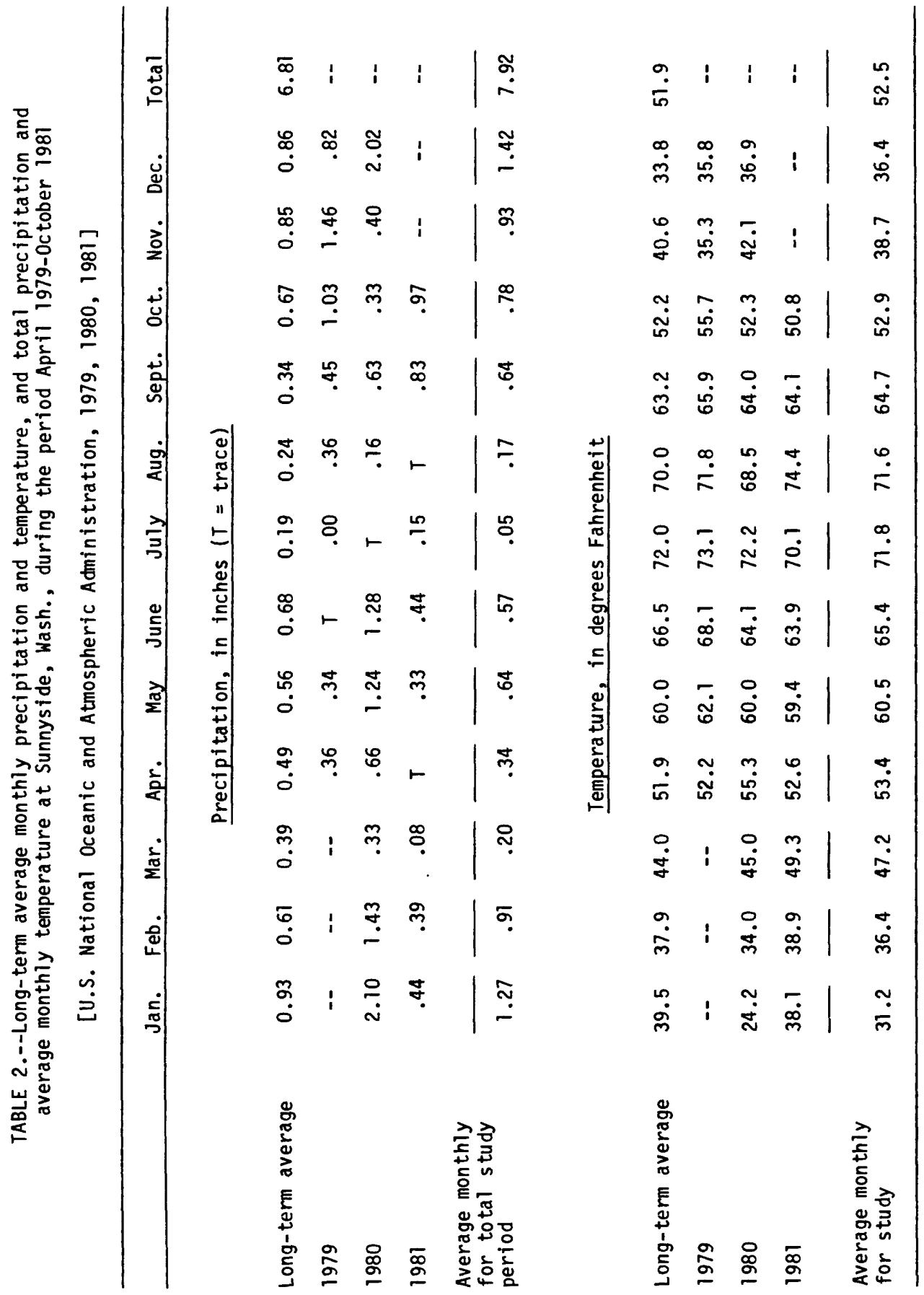


TABLE 3.--Crops grown and drainage area data for Drains 61.0,60.7, 59.6, and 59.4, in the DID 18 Drainage near Sunnyside, Wash., for the period 1979-81

\begin{tabular}{|c|c|c|c|c|c|c|c|c|c|c|c|c|}
\hline \multirow{3}{*}{ Crop grown } & \multicolumn{12}{|c|}{ Percentage of crops grown in given drains, 1979-81 } \\
\hline & \multicolumn{3}{|c|}{ Drain 59.4} & \multicolumn{3}{|c|}{ Drain 59.6} & \multicolumn{3}{|c|}{ Drain 60.7} & \multicolumn{3}{|c|}{ Drain 61.0} \\
\hline & 1979 & 1980 & 1981 & 1979 & 1980 & 1981 & 1979 & 1980 & 1981 & 1979 & 1980 & 1981 \\
\hline Alfalfa & 4.9 & 4.9 & ns & 4.8 & 10.2 & 8.1 & 1.3 & 0.6 & 0.6 & 21.3 & 21.3 & 21.3 \\
\hline Asparagus & 4.1 & 5.0 & 3.3 & 3.3 & 3.3 & .6 & 6.7 & 6.7 & 6.7 & ns & ns & ns \\
\hline Beans & 14.9 & 10.5 & 22.0 & 11.6 & 14.5 & 15.0 & 3.5 & 3.5 & ns & ns & ns & ns \\
\hline Corn & 23.8 & 37.1 & 8.2 & 5.6 & 17.8 & 8.9 & 24.0 & 9.6 & ns & 17.2 & 1.8 & ns \\
\hline Fallow & 6.6 & 6.6 & 6.6 & 15.8 & 15.7 & 15.7 & 13.4 & 12.6 & 12.6 & 22.1 & 22.1 & 22.1 \\
\hline Grapes & ns & ns & ns & 8.9 & 8.9 & 13.2 & 21.3 & 21.3 & 24.1 & 25.0 & 25.0 & 21.6 \\
\hline Mint & 37.1 & ns & 5.2 & 22.1 & 2.4 & 1.0 & ns & ns & 10.3 & ns & ns & 5.3 \\
\hline Orchard & ns & ns & 3.5 & ns & 3.1 & 5.9 & 11.7 & 11.7 & 11.7 & 5.5 & 5.5 & 8.9 \\
\hline Pasture & ns & ns & ns & 1.5 & 2.3 & 1.5 & 2.5 & 2.5 & 2.5 & 7.1 & 7.1 & 7.1 \\
\hline Sma1l grain & 8.6 & 35.9 & 51.2 & $\underline{26.4}$ & 21.8 & 30.1 & 15.6 & 31.5 & 31.5 & 1.8 & 17.2 & 13.7 \\
\hline Total & 100 & 100 & 100 & 100 & 100 & 100 & 100 & 100 & 100 & 100 & 100 & 100 \\
\hline
\end{tabular}

Drainage area data (acres)

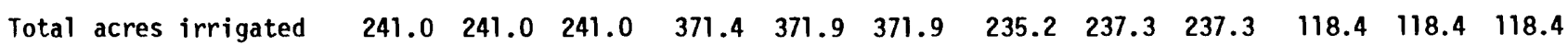

Noncropped

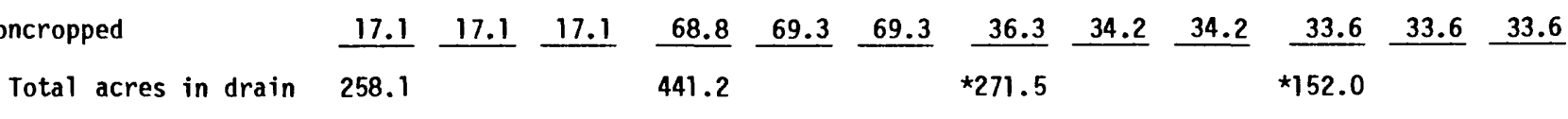

*Excluding dry land above Roza Canal crossing.

ns - not significant 


\section{METHODS OF DATA COLLECTION AND ANALYSIS}

\section{Monitoring Methods}

At the outflow points (sites 1, 2, 3, and 4; fig. 1, table 1) on each drain the Geological Survey established stream-gaging stations with artificial plywood controls and automatic water-level recorders to monitor the stage continuously. The procedure and methods of stream gaging and record computation are described in various Geological Survey reports and memoranda. The most common methods used in this study are outlined by Carter and Davidian (1968) and Buchanan and Somer (1969). Measurements of water flow into the project area were obtained from records provided by the Roza Irrigation District.

Automatic sediment samplers were installed at the outflow points from each drain (sites 1, 2, 3 and 4). The automatic sampler used was the Manning Model S4050 sampler, manufactured by the Manning Corp. ${ }^{\mathrm{a}}$ The samplers were programmed to sample on a 4-hour frequency. The six samples collected each day were then composited by equal volume. Collection of equal volumes was accomplished by adjusting the fill-sensor level in the automatic-sampler collection chamber. The orifice of the sampler was placed in a turbulent area below the gaging flume to dampen the effects of stream velocity and provide mixing. Several times throughout the irrigation season, calibration samples were taken at the artificial controIs. The calibration samples were collected by using a US series DH-48 suspended-sediment sampler, and were used to correct those obtained by the automatic sampler. Because the nozzle of the DH-48 sampler reached all the way to the bottom of the artificial control, the samples represent the total sediment concentration and discharge. Manual sediment sampling technique--by EWI method--and the methods used by the U.S. Bureau of Reclamation Sediment Laboratory to determine the sediment concentration and particle size are described by Guy (1969) and Guy and Norman (1970).

To monitor sediment discharge entering the study area from the Roza Canal, a sampling station was established at the diversion located at mile 59.9 (site 7). Suspended-sediment samples were obtained about five times a week (table 13, end of report). To adjust the sediment concentrations at site 7 to reflect the concentrations at the several points of diversion from the Roza Canal, 20 periodic sediment-concentration samples were collected at sites $5,6,8,9$, and 10 (see fig. 1), and these data were used to adjust the values obtained at site 7 (table 14, end of report). The adjustment factor averaged 0.97 for the 20 samples collected.

aThe use of the brand name in this report is for identification purposes only and does not constitute endorsement by the U.S. Geological Survey. 
Because water-discharge data are also necessary to obtain the inflow sediment discharge, diversion records kept by the Roza Irrigation District were analyzed (tables $8 \mathrm{a}-\mathrm{d}$ ). Each diversion at the canal may supply water to more than one drain; therefore, the flow data had to be broken into segments to reflect water flow into each separate drain, and to determine what water flowed from the study area. To keep the calculations to a minimum, monthly summaries of water flow were multiplied by monthly mean adjusted sediment concentration for the diversion at site 7 .

During the nonirrigation periods, when WDOE observers were not available, the automatic samplers were shut down except during periods when runoff from rain or snowmelt was suspected. The samplers were susceptible to freezing because the shelters were not heated. Except for significant runoff periods, samples were taken about once monthly. Fortunately, only one significant period of runoff occurred during the nonirrigation period, and that was for the partially sampled period February 16 to $21,1980$.

Periodically, samples (table 12, end of report) were obtained at the culverts (sites 11-18) on SLI Road where the drains cross, using a DH-48 sampler. These samples were used to estimate sediment discharge north of SLI Road.

When sediment samples were obtained by hand, water temperatures were obtained. Tables 12,13, and 14 list these data for the miscellaneous sites and diversion points on the canal. The temperatures for outflow points on the drains are listed as once-daily temperature in tables lla to $11 \mathrm{~d}$ (end of report).

Sediment samples were collected above and below a sediment pond located on Drain 60.7 (sites 19-22) about half a mile above the gaging station at site 20 to estimate the trap efficiency of the pond.

Runoff north of the Roza Canal only occurred during a storm during February 1980, and was sampled at two sites (23 and 24). These samples, though sparse, were used to estimate the storm contribution of sediment above Roza Canal to Drains 60.7 and 61.0 .

Water-temperature data (tables 11a-d), which were obtained when the sampling sites were visited, were analyzed by harmonic analysis. The method was developed by Ward (1963) and refined by Collings (1969) and Steele (1974).

Irrigation seasons and nonirrigation seasons were deter mined by an analysis of the flow records for Roza Canal kept by the Roza Irrigation District. The time when water was being discharged from the canal was considered the irrigation season and the time when no water was being discharged was considered the nonirrigation season. The periods of irrigation and nonirrigation are listed as follows:

1979 irrigation season 1979-80 nonirrigation season 1980 irrigation season 1980-81 nonirrigation season 1981 irrigation season
Apr. 1 to Nov. 6

Nov. 6, 1979, to Apr. 4, 1980

Apr. 5 to Oct. 22

Oct. 23, 1980, to Mar. 27, 1981

Mar. 27, 1981, to Oct. 25, 1981 
As stated on page 3, the Washington State Department of Ecology, with the aid and consultation of the Technical Advisory Committee on Irrigation, the Geological Survey, and Washington State University, has adopted the lmhoff Cone technique to monitor the sediment from irrigation fields and return flow in irrigation drains. The method has been used for years in sewage treatment plants to determine the settleable solids present, where the normal time allowed for the material to settle is 45 minutes. The time selected for monitoring irrigation water by the lmhoff Cone was 15 minutes.

The lmhoff Cone used by WDOE is plastic, cone-shaped, $40.8 \mathrm{~cm}$ high, $9.3 \mathrm{~cm}$ in diameter at the top, and holds 1 liter of liquid. The technique is to collect a depth-integrated sample of l liter in volume, pour it into the cone, and let the water-sediment mixture settle for 15 minutes. The cone is graduated in milliliters, and the amount of settleable material per 1-liter volume is read by holding the cone in an upright position and reading through the graduated transparent plastic. The results are reported as settleable solids in milliliters per liter per 15 minutes.

The use of lmhoff Cones to measure water quality in irrigation return flows was felt to have the following advantages:

1. The cones are inexpensive, and no laboratory analysis is required.

2. The procedure is simple and can be easily taught to farmers and laypersons. Farmers faced with meeting a performance standard can easily determine where they stand.

3. The procedure is fast. Samples can be measured within 15 minutes of collection, and no storage or transporation is required.

4. The parameter measured (for example, settleable solids) is easily understood and is assumed to be related to soil loss from the farm. 


\section{Error Analysis}

During several 24-hour periods (diel studies) for each irrigation season, the outflow monitor stations on the drains (sites 1, 2, 3, and 4) and the daily-inflow station (site 7) on Roza Canal were sampled on a bi-hourly and hourly basis (tables $10 \mathrm{a}-\mathrm{d}$, end of report), and analyzed discretely. The samples were used to estimate the daily variation error at the monitor stations at sites $1,2,3$, and 4 introduced by compositing the automatic samples by equal volume, and the daily variation error caused by sampling site 7 only once per day.

The estimated error analysis for the daily values was arrived at by calculating the daily variability in the sediment discharge and combining that with other sources of uncertainty--such as sampling error, laboratory error, and water discharge error-using principles of propagation of uncertainty (Baird, 1962) into a single estimated confidence limit for the data. Confidence limits were discussed by Boucher and Fretwell (1982).

The estimated confidence limits of sediment yields and net outflows from a basin were obtained using standard statistical methods for determining the uncertainty of sums and differences. The concept of "Least Significant Difference" is described by Arkin and Colton (1970).

Standard statistical methods of analysis of co-variance and regression techniques are described in various statistical textbooks. The methods used for this report are described by Riggs (1968). 


\section{SEDIMENT TRANSPORT}

\section{Inflow}

During the 1979 irrigation season, water in Roza Canal was shut off from September 8 to October 17, and turned back on again October 18 to November 6 . The surface-water inflow to the study area during the 1979 irrigation season was about 80 percent of the average for the 1980 and 1981 irrigation seasons (table 4). A small but unknown ground-water pumpage affects inflow to the area.

The sediment inflow for the 1979 irrigation season was 298 tons, compared with only 119 tons for the 1980 and 88 tons for the 1981 irrigation seasons (table 5). The discharge-weighted-mean sediment concentration for inflowing water for the 1979 irrigation season was $88-120 \mathrm{mg} / \mathrm{L}$, about three times that for the 1980 irrigation season (31-33 mg/L), and about four times that for the 1981 irrigation season $(23-24 \mathrm{mg} / \mathrm{L})$. The discharge-weighted mean sediment concentration, $\overline{\mathrm{C}}$, is calculated by using the equation

$$
\overline{\mathrm{C}}=\frac{\text { seasonal sediment discharge }}{\text { seasonal water discharge } \times 0.0027} \text {, }
$$

where $\mathbf{0 . 0 0 2 7}$ is a factor to convert to tons per day.

Data from the two sampling sites (23 and 24, fig. 1) above Roza Canal were used to calculate water-sediment inflow to Drains 60.7 and 61.0 from the nonirrigated areas above the Roza Canal. The two sites had flow only during the stor $m$ runoff, February 16-21, 1980. The inflow was estimated to be 60 tons; 57 tons was contributed to Drain 61.0 (site 24) and 3 tons to Drain 60.7 (site 23). There is no inflow from nonirrigated lands into Drains 59.6 and 59.4, because all the drainage of these two drains lies south of Roza Canal. Inflow from the nonirrigated area during the February 1980 storm into Drain 61.0 was about 3.5 times more than moved past the outlet monitoring station during the study. The inflow from the nonirrigated area to Drain 60.7 during the February 1980 storm period was estimated to be about 0.4 percent of the sediment discharged past the outlet (site 2) during the study. The sediment inflows to the four drains, including that for the February 1980 storm, are listed in the following table.

\begin{tabular}{lllll} 
& \multicolumn{3}{c}{ Drain } \\
$\begin{array}{l}\text { Sediment inflow for the study } \\
\text { (adapted from table 5), in tons }\end{array}$ & $\underline{61.0}$ & $\underline{60.7}$ & $\underline{59.6}$ & $\underline{59.4}$ \\
$\begin{array}{l}\text { Discharge-weighted mean sediment concen- } \\
\text { tration, in milligrams per liter }\end{array}$ & 120 & 139 & 181 & 125 \\
$\begin{array}{l}\text { Estimated sediment inflow, in tons, for } \\
\quad \text { February 1980 storm }\end{array}$ & 90 & 49 & 45 & 55 \\
\end{tabular}


TABLE 4.--Water outflows and inflows in four subbasins of the DID-18 Drain

\begin{tabular}{|c|c|c|c|c|c|c|c|}
\hline \multirow{3}{*}{$\begin{array}{l}\text { Site } \\
\text { No. }\end{array}$} & \multirow{3}{*}{$\begin{array}{l}\text { Selected site or } \\
\text { inflow to drain }\end{array}$} & \multicolumn{6}{|c|}{ Irrigation season } \\
\hline & & \multicolumn{2}{|c|}{1979} & \multicolumn{2}{|c|}{1980} & \multicolumn{2}{|c|}{1981} \\
\hline & & $\mathrm{ft}^{3} / \mathrm{s}-$ days & acre-feet & $\mathrm{ft}^{3} / \mathrm{s}-\mathrm{days}$ & acre-feet & $f t^{3} / s-$ days & acre-feet \\
\hline 1 & Drain 61.0 outflow & 83.03 & 165 & 55.68 & 110 & 81.42 & 161 \\
\hline 2 & Drain 60.7 outflow & 211.02 & 418 & 236.88 & 470 & 282.96 & 561 \\
\hline 3 & Drain 59.6 outflow & 314.7 & 624 & 419.4 & 832 & 361.98 & 718 \\
\hline \multirow[t]{5}{*}{4} & Drain 59.4 outflow & 60.43 & 120 & 47.71 & 95 & 67.29 & 133 \\
\hline & Drain 61.0 inflow & 130 & 270 & 170 & 330 & 190 & 380 \\
\hline & Drain 60.7 inflow & 320 & 630 & 370 & 740 & 410 & 820 \\
\hline & Drain 59.6 inflow & 450 & 890 & 590 & 1,170 & 500 & 1,000 \\
\hline & Drain 59.4 inflow & 260 & 510 & 300 & 590 & 320 & 650 \\
\hline \multirow{3}{*}{$\begin{array}{l}\text { Site } \\
\text { No. }\end{array}$} & \multirow{3}{*}{$\begin{array}{l}\text { Selected site or } \\
\text { inflow to Drain }\end{array}$} & \multicolumn{4}{|c|}{ Nonirrigation season } & & \\
\hline & & \multicolumn{2}{|c|}{$1979-80$} & \multicolumn{2}{|c|}{$1980-81$} & & \\
\hline & & $\mathrm{ft}^{3} / \mathrm{s}-$ days & acre-feet & $\mathrm{ft}^{3} / \mathrm{s}-$ days & acre-feet & & \\
\hline 1 & Drain 61.0 outflow & $\star 12.81$ & 26 & 12.73 & 25 & & \\
\hline 2 & Drain 60.7 outflow & $\star 161.84$ & 319 & 107.91 & 214 & & \\
\hline 3 & Drain 59.6 outflow & $\star 229.67$ & 456 & 209.34 & 415 & & \\
\hline \multirow[t]{5}{*}{4} & Drain 59.4 outflow & $\star 17.28$ & 34 & 7.83 & 16 & & \\
\hline & Drain 61.0 inflow & $\star \star 5$ & $\star \star 10$ & 0 & 0 & & \\
\hline & Drain 60.7 inflow & $\star \star 1$ & $\star \star 2$ & 0 & 0 & & \\
\hline & Drain 59.6 inflow & 0 & 0 & 0 & 0 & & \\
\hline & Drain 59.4 inflow & 0 & 0 & 0 & 0 & & \\
\hline
\end{tabular}

*Includes nonirrigation period September 8 to 0ctober 17 .

**This is estimated inflow from the dryland portion above Roza Canal. The canal did not flow during the nonirrigation periods. 


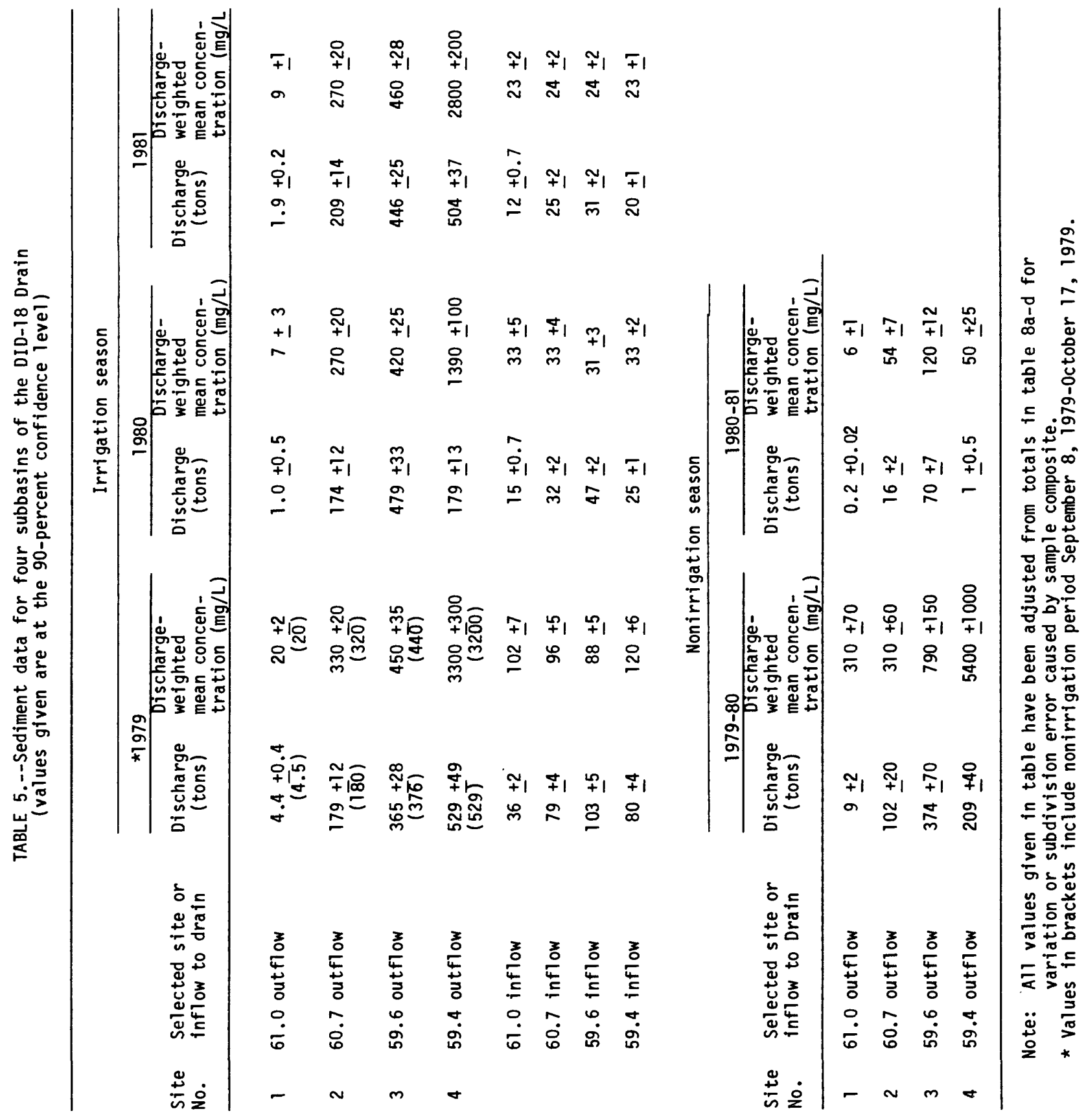


Boucher and Fretwell (1982) reported a discharge-weighted mean sediment concentration of $55 \mathrm{mg} / \mathrm{L}$ in the Roza Canal at DID-18 (site C) during the 1976 irrigation season. The average concentrations of suspended sediment in the inflow water from Roza Canal were 99, 32, and $24 \mathrm{mg} / \mathrm{L}$ during the 1979, 1980, and 1981 irrigation seasons.

Sediment concentrations increased in some streams in the Yakima River basin and other parts of east-central Washington due to the ash fall from the eruption of Mount St. Helens on May 18, 1980. Although samples at the diversion on Roza Canal at mile 59.9 (site 7) were not taken from May 17-21, sediment concentrations increased from $35 \mathrm{mg} / \mathrm{L}$ on May 16 to 69 and $83 \mathrm{mg} / \mathrm{L}$ on May 22 and 23 , respectively. The increase may have been due to ash in the canal water.

\section{Outflow}

Water outflow during the study was greatest for Drain 59.6 (table 4), which drains more irrigated area than any of the other three sites (table 3). Water outflow was least for Drain 59.4, except during the 1979-80 nonirrigation season when Drain 59.4 discharged 34 acre- $\mathrm{ft}$ and Drain 61.0, 26 acre-ft. Base flows tended to be somewhat greater during the 1979-80 nonirrigation season than during the 1980-81 nonirrigation season, even though the nonirrigation season of 1979-80 was shorter than that of 1980-81. Figure 2 illustrates the monthly distribution of sediment discharge from the four drains during the study.

During the study, 16.6 tons of sediment was transported past site 1 on Drain 61.0, of which 8.5 tons was transported during the runoff of February 16-21, 1980. In other words, 51 percent of the 3-year sediment discharge of Drain 61.0 was transported during this one storm. The following table lists the sediment discharge during the study and during the February 16-21, 1980, runoff and the discharge-weighted mean sediment concentration:

\begin{tabular}{|c|c|c|c|c|}
\hline & & Drain & & \\
\hline & 61.0 & 60.7 & $\underline{59.6}$ & 59.4 \\
\hline $\begin{array}{l}\text { Outflow sediment discharge, in tons, } \\
\text { during the study }\end{array}$ & 16.6 & 681 & 1,750 & 1,420 \\
\hline $\begin{array}{l}\text { February } 1980 \text { storm sediment discharge, } \\
\text { in tons (percentage of the sediment } \\
\text { transported during the study in } \\
\text { parentheses) }\end{array}$ & $\begin{array}{r}8.5 \\
(51)\end{array}$ & $\begin{array}{l}73.2 \\
(11)\end{array}$ & $\begin{array}{l}290 \\
(17)\end{array}$ & $\begin{array}{l}205 \\
(14)\end{array}$ \\
\hline $\begin{array}{l}\text { Discharge-weighted mean sediment } \\
\text { concentration during the study, } \\
\text { in milligrams per liter }\end{array}$ & 25 & 252 & 422 & 2,620 \\
\hline $\begin{array}{l}\text { February } 1980 \text { storm discharge-weighted } \\
\text { mean sediment concentration, } \\
\text { in milligrams per liter }\end{array}$ & 580 & 3,200 & 9,490 & 9,650 \\
\hline
\end{tabular}




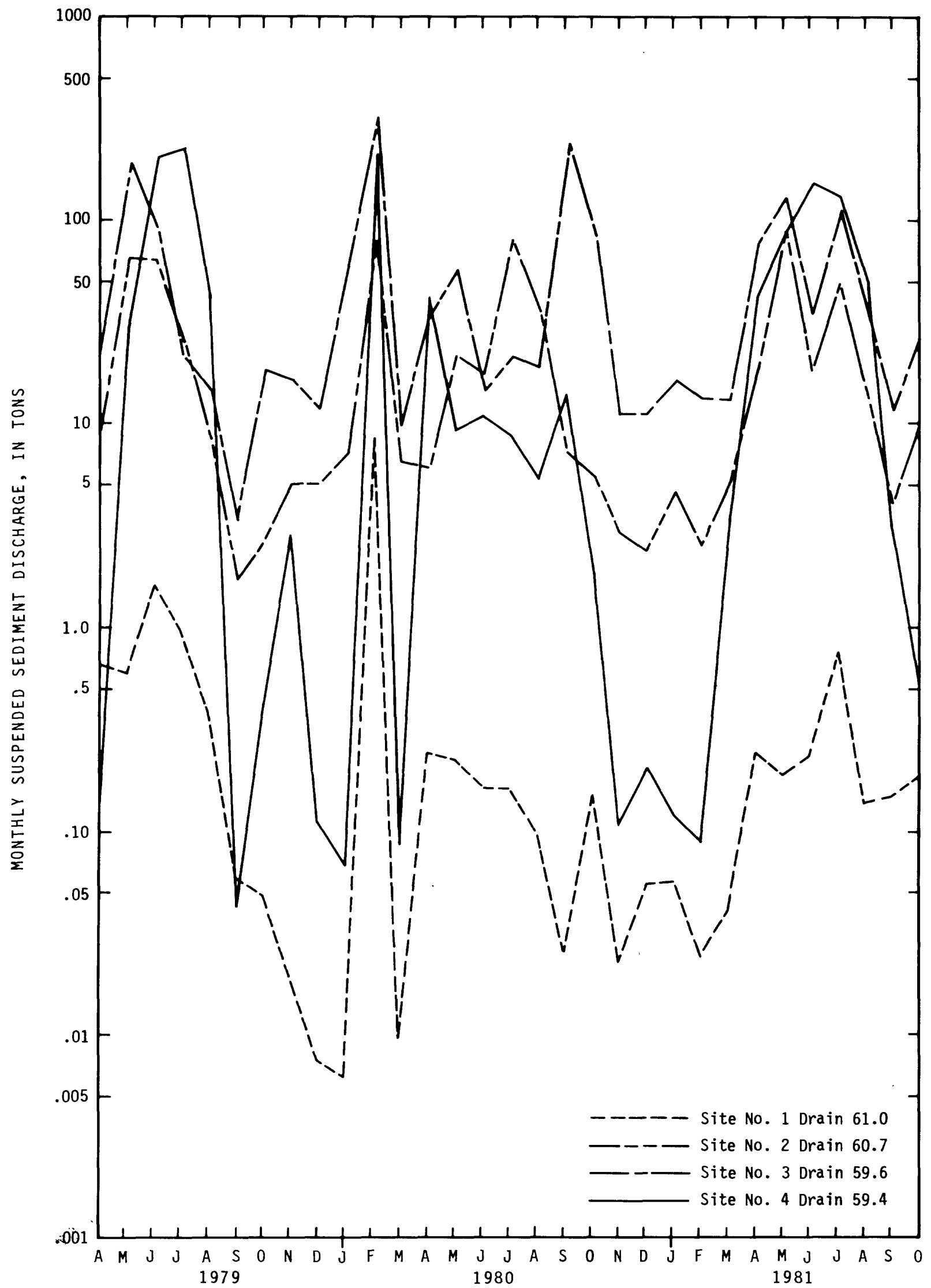

FIGURE 2.--Monthly sediment discharge for Drains $61.0,60.7,59.6$, and 59.4, near Sunnyside, Wash., for the period April 1979 to October 1981. 
The importance of sampling storms during the nonirrigation season is illustrated in the above table. A large variation in sediment transport occurs in the four drains during any one storm. A large percentage of the annual sediment discharge may be transported during a single storm, as it was for Drain 61.0 in 1980 .

For Drain 59.4, discharge-weighted mean sediment concentrations for the three irrigation seasons varied by $1,910 \mathrm{mg} / \mathrm{L}$. For the other three drains, discharge-weighted mean concentrations varied only $13 \mathrm{mg} / \mathrm{L}, 60 \mathrm{mg} / \mathrm{L}$, and 40 $\mathrm{mg} / \mathrm{L}$ for Drains 61.0, 60.7, and 59.6, respectively. This indicates that sediment erosion in Drain 59.4 was more variable from year to year than in the other three drains.

Discharge-weighted mean sediment concentration during the study at the sampling site on Drain 61.0 was 105 times less than in Drain 59.4, 17 times less than in Drain 59.6, and 10 times less than in Drain 60.7. During the 1979-80 nonirrigation season, the discharge-weighted mean concentration in Drain 61.0 was still equal to or less than that in the other three drains, even though much more water flowed into this drain from above Roza Canal during the nonirrigation period.

\section{Sediment Yields}

To determine the sediment yield, a mass balance of the sediment loads was first determined from the equation

$$
\mathrm{S}=\mathrm{L}_{\text {out }}-\mathrm{L}_{\mathrm{in}}
$$

where $\mathrm{S}$ is the change in sediment storage in the basin, $\mathrm{L}_{\text {out }}$ is the sediment flowing out of a basin, and $L_{i n}$ is the sediment flowing into a basin. Sediment yield is then defined as $S$ divided by drainage area. In a report about water quality of the Bear Creek Basin, Jackson County, Oregon, Wittenberg and McKenzie (1980) stated, "The irrigation method and crop type generally will determine whether a farm plot acts as a concentrator or diluter of constituents in water or as a source or sink. Explanations of these terms are given below:

\section{CONCENTRATION (weight/volume)}

Outflow greater than inflow = Concentrator

Outflow less than inflow $=$ Diluter

$$
\text { LOAD (weight/time) }
$$

Outflow greater than inflow = Source

Outflow less than inflow $=\underline{\text { Sink." }}$ 
From the above definitions, when $\mathrm{S}$ is positive the basin becomes a source for sediment, and when $S$ is negative the basin becomes a sink for sediment. Figure 3 shows the change in sediment storage, including the uncertainty, for the four drains and table 6 lists the sediment yields.

Drain 61.0 was a sink for sediment for all periods except the 1980-81 nonirrigation season. An estimated 104 tons of sediment was deposited in the 61.0 basin. The natural erosion potential for Drain 61.0 is as great as or greater than for the other drains on the basis of soil types and slopes. Therefore, factors such as cropping, water management, and depositional processes are assumed responsible for Drain 61.0 acting as a sink for sediment. Even during the runoff period of February 16-21, Drain 61.0 acted as a sink for sediment below Roza Canal, in that an estimated 57 tons was transported into the basin at Roza Canal crossing and only 9 tons was transported out of the basin. From this, about 48 tons was deposited along the drainageway, to be available for transport at a later time. During the 1980-81 nonirrigation season, Drain 61.0 acted (barely) as a source of sediment, since $\mathbf{0 . 2}$ ton was transported out of the basin; yet this is to be expected, because no surface inflow (other than minor precipitation) occurred during this period.

Comparing the outflow discharge-weighted mean sediment concentrations from the three irrigation seasons shows that outflow sediment concentrations in Drain 61.0 were 3 to 5 times less than the inflow sediment concentrations. Drain 61.0 acts as a diluter. In the other three drains, outflow sediment concentrations were 3 to 120 times greater than inflow concentrations; these drains act as concentrators.

Figure 3 shows the change in sediment storage as obtained by the mass balance equation. If the range in the least-significant difference overlaps, then there is not a statistically significant difference in the change in sediment storage from one year to the next. For example, the least-significant difference overlaps for Drain 59.6 for the 1980 and 1981 season; therefore, the difference there is not statistically significant.

There is a increasing trend in sediment coming out of storage in Drain 60.7, the loss has increased each irrigation season (101, 142, and 184 tons for the 1979 , 1980, and 1981 irrigation seasons, respectively). For Drain 59.6, the 1980 and 1981 irrigation seasons were not significantly different, but both years had about 65 percent greater yield than the 1979 irrigation season, differing by an average of 424 versus 273 tons coming out of storage.

The change in sediment storage from Drain 59.4 was not significantly different in the 1979 and 1981 irrigation season, averaging 466 tons. However, change in storage in the 1980 irrigation season, 154 tons, was significantly less (by about 3 times). 


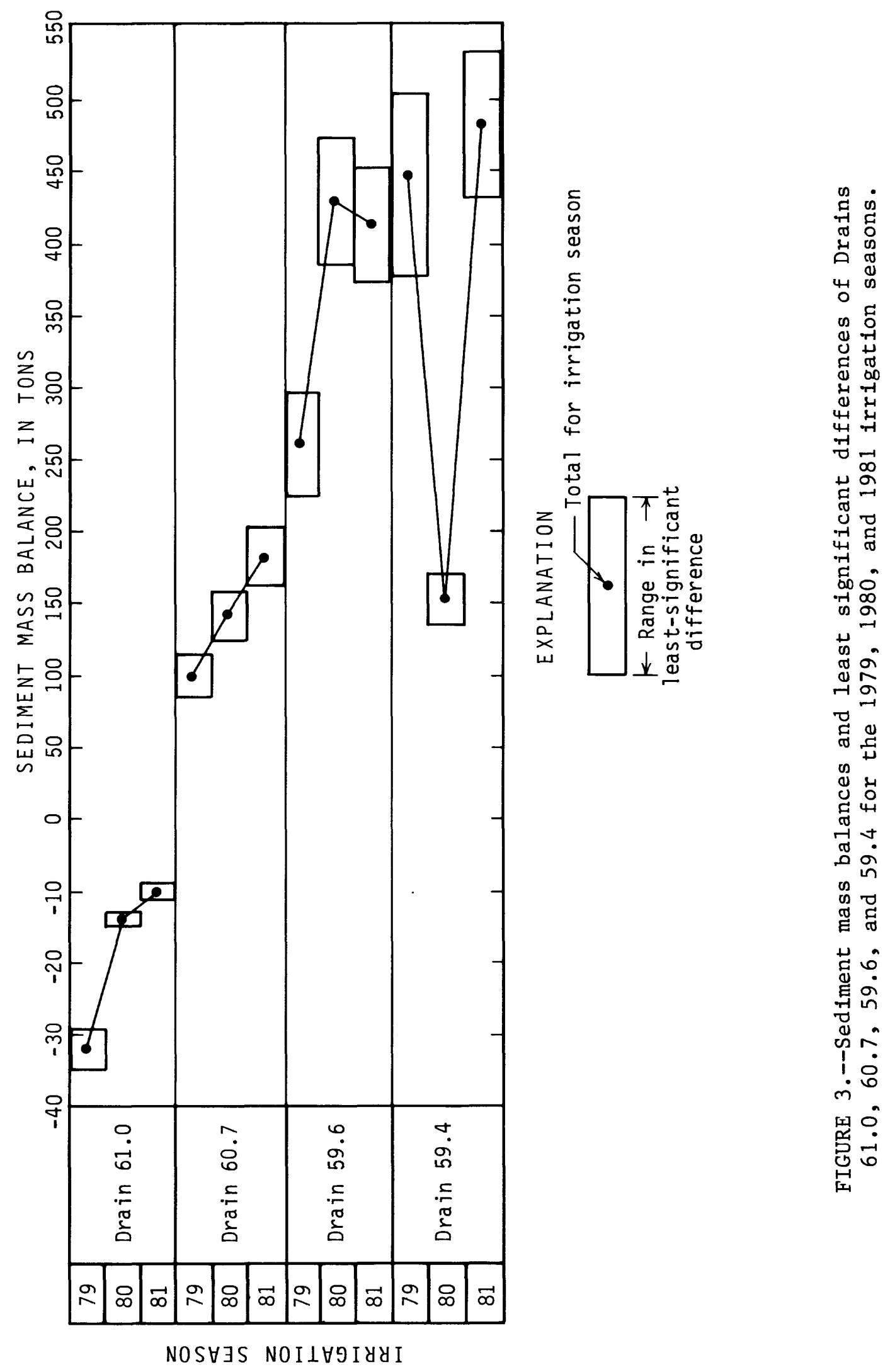


TABLE 6.--Sediment yields for four drains in the DID-18 Drain

\begin{tabular}{|c|c|c|c|c|c|}
\hline Subbasin & $\begin{array}{c}1979 \\
\text { irrigation } \\
\text { season } \\
\text { tonsa } \\
\text { tons/acreb }\end{array}$ & $\begin{array}{c}1979-80 \\
\text { nonirrigation } \\
\text { season } \\
\text { tonsa } \\
\text { tons/acreb }\end{array}$ & $\begin{array}{c}1980 \\
\text { irrigation } \\
\text { season } \\
\text { tonsa } \\
\text { tons/acreb }\end{array}$ & $\begin{array}{c}1980-81 \\
\text { nonirrigation } \\
\text { season } \\
\text { tonsa } \\
\text { tons/acreb }\end{array}$ & $\begin{array}{c}1981 \\
\text { irrigation } \\
\text { season } \\
\text { tonsa } \\
\text { tons/acreb }\end{array}$ \\
\hline Drain 61.0 & $\frac{-32}{-0.27}$ & $\frac{c_{-48}}{-0.41}$ & $\frac{-14}{-0.12}$ & $\frac{0.2}{0.002}$ & $\frac{-10}{-0.09}$ \\
\hline Drain 60.7 & $\frac{101}{0.43}$ & $\begin{array}{l}c 99 \\
0.43\end{array}$ & $\frac{142}{.60}$ & $\frac{16}{0.07}$ & $\frac{184}{0.78}$ \\
\hline Drain 59.6 & $\frac{273}{0.74}$ & $\frac{374}{1.0}$ & $\frac{432}{1.2}$ & $\frac{70}{0.19}$ & $\frac{415}{1.1}$ \\
\hline Drain 59.4 & $\frac{449}{1.9}$ & $\frac{209}{0.87}$ & $\frac{154}{0.64}$ & $\frac{1}{0.004}$ & $\frac{484}{2.0}$ \\
\hline $\begin{array}{l}\text { Total for } \\
\text { four basins }\end{array}$ & $\frac{791}{.82}$ & $\frac{634}{.66}$ & $\frac{714}{.74}$ & $\frac{87}{.09}$ & $\frac{1073}{1.1}$ \\
\hline
\end{tabular}

asediment outflow minus inflow.

byield is equal to outflow minus inflow divided by irrigated acres.

CNet for irrigated portion. 
Sediment yield for the DID-18 Drain during the 1976 irrigation season (Boucher and Fretwell, 1982) was about 7.1 tons per acre. This is about 3.5 times greater than the yield for Drain 59.4, which was 2.0 tons per acre (table 6) during the 1981 irrigation season, the greatest yield determined for any of the drains during the 1979-81 period. Because DID-18 Drain was not sampled during the 1979-80 irrigation season, there were no data to determine if the yield from the total DID-18 drainage would be lower than that reported during the 1976 irrigation season. However, most of the difference in sediment discharge between the 1976 and 1981 irrigation seasons is due to the outflows of sediment from DID 18 in the Sunnyside Canal during 1976. The sediment yields in tons per acre (Boucher and Fretwell, 1982) for the 1976 irrigation season for the six drains in the Sulphur Creek basin were as follows:

$\begin{array}{ll}\text { DID-3 and Washout Drain } & 1.9 \\ \text { DID-1 8 Drain } & 7.1 \\ \text { Black Canyon Creek } & 2.7 \\ \text { DID-9 Drain } & 0.5 \\ \text { Sulphur Creek Wasteway (entire basin) } & 1.9\end{array}$

Comparison of this table with the study-drain yields (shown as denomininators in table 6) showed that sediment yields were generally greater in the Sulphur Creek basin during the 1976 irrigation season than those reported for the 1979-81 irrigation season in Drains 60.7, 59.6, and 59.4. Yields in Drain 61.0, which acts as a sink for sediment, were considerably less.

The sediment concentration in DID-18 Drain at the outflow point (site B, fig. 1) was $1,200 \mathrm{mg} / \mathrm{L}$ for the 1976 irrigation season (Boucher and Fretwell, 1982) and 589 $\mathrm{mg} / \mathrm{L}$ for the 1974 irrigation season $\left(\mathrm{CH}_{2} \mathrm{M}\right.$ Hill, 1975), or a discharge of 11,000 tons for 1976 and 5,430 tons for 1974. This indicates that sediment discharge can vary greatly between irrigation seasons, depending on amount of water applied (including precipitation), type of crops grown, slope of the land, and management practices.

A study of the periodic data (table 12) collected from sites 11 to 18 (fig. 1), where the drainages are cut into by SLI Road, indicates that sediment yields in the area north of SLI Road ranged from 50 to 200 percent of sediment yields for the entire drainage basins above the outflow sites. However, there is a wide variance in the sediment discharge, and good estimates could not be made. 
The eruption of Mount St. Helens on May 18, 1980, created a large ash fall in portions of eastern Washington. The effect was noticeable in samples taken at a project in the Royal Slope area in DW272A Drain near Royal City, about 43 miles to the northeast of the study area, where the ashfall was about 20 millimeters (Sarna-Wojcicki and others, 1981). Sediment concentration increased from $294 \mathrm{mg} / \mathrm{L}$ to $8,020 \mathrm{mg} / \mathrm{L}$ in Drain DW272A near Royal Camp, and from 179 to $5,980 \mathrm{mg} / \mathrm{L}$ for DW272Al, a tributary drain near Royal Camp (Boucher and O'Neil, written commun., 1982). The ashfall in the DID-18 basin study area was about 1 millimeter in depth, and the effect on sediment discharge from the ashfall was minimal. The 4 -hour samples collected by the automatic samplers were analyzed separately; only Drain 61.0 indicated any discernible increase (an increase of $17 \mathrm{mg} / \mathrm{L}$ in 4 hours) in sediment concentration (see table $10 \mathrm{a}$ at the end of report). Most diel samples taken on Drain 61.0 do not show this large an increase in the 2-hour and 1-hour samples, so it is possible that the increase could be attributed to ashfall. Detection of sediment increases caused by ashfall were not discernible in the other drains because of the masking effects of irrigation return flow.

\section{$\underline{\text { Factors Affecting Sediment Yields }}$}

\section{Irrigation and Land Use}

The amount of sediment transported by surface irrigation for certain types of crops is affected by many variables, such as soil types, land slopes, irrigation management practices, and types of irrigation (surface versus sprinkler). A detailed study of the methods used to irrigate the various crops was beyond the scope of this project. However, WDOE has studied in detail the irrigation methods and Best Management Practices (BMP) in the four drains. According to Joan Thomas (WDOE, written commun., 1982) there were no statistical differences between the Imhoff Cone readings (to be discussed later) for the three irrigation seasons. Therefore, by agreement with the WDOE, the Geological Survey did not make a detailed analysis of the relation of BMP to sediment transport in the four basins, the third objective of the study.

Although no conclusion was made about the impact of BMP on sediment transport, it is noted that basins with the greatest percentage of row crops (asparagus, beans, corn, mint, and small grains) have the greatest sediment yields. A study of the types of crops grown indicates that sediment discharge tends, as a rule, to increase when crops types conducive to erosion are grown. Drains 59.4, 59.6, and 60.7 have a higher proportion of row crops, which are conducive to erosion, than does Drain 61.0. Boucher and Fretwell (1982) noted that sediment yields relate best to land slopes and, to some degree, to land in orchards. However, land slopes in the drains of this study are somewhat similar and the area in orchards is fairly small (table 3 ). 
Management practices in Drain 61.0 were implemented prior to this study and appear to be different. Over 50 percent of Drain 61.0 area is either in fallow, alfalfa, or pasture. Wittenberg and McKenzie (1980) found that pastures in the Bear Creek Basin, Oregon, tended to trap moving sediment and thus act as sinks for sediment. Irrigation of alfalfa results in much the same sediment-trapping characteristics as irrigation of pasture.

One other reason why Drain 61.0 acts as a sink for sediment may be that after sediment is transported from the farm field it is deposited along the stream channel to be transported out of the basin at a later time. Data obtained during the highest runoff period in February 1980 indicated that more sediment was transported into the irrigated portion of the basin below Roza Canal than moved out (an estimated 48 tons, see pages 19 and 20). This material was probably deposited along the stream channel. Thus, the sediment delivery ratio (sediment eroded from farm fields divided by sediment transported past a given point) in Drain 61.0 is much lower than in the other drains.

Data supplied by the Soil Conservation Service (written commun., 1982) indicates a higher proportion (at least 60 percent) of sprinkler irrigation in Drain 61.0 than in the other three drains. Sprinkler irrigation is generally regarded as least likely to cause erosion. The use of sprinklers in combination with good management practices (resulting in less direct runoff) may be one more reason for the drain to act as a sink for sediment. However, the way sprinklers are managed is very important. Above SLI Road on Drain 59.4 there is a pivot sprinkler that covers about 68 acres, or about 50 percent of the area. Much of the erosion above SLI Road in Drain 59.4 came from the sprinkler-irrigated area. If not managed properly, wastewater from sprinkler systems may cause as much or more erosion than surface irrigation.

Data supplied by Cliff Eckhardt, MIP Project Manager (written commun., 1982), indicates very little change in BMP throughout the three irrigation seasons. A small increase in the use of sprinklers was introduced in the Drain 61.0 basin (about 25 acres), but other than this, very little change was made in sprinkler systems during the 1979 and 1981 irrigation seasons. Some sprinkling, mostly in Drain 60.7, was accomplished by pumping water directly out of the drains rather than using Roza Canal water, but the total amount of irrigated acreage, both surface and sprinkling, probably remained about the same during the 3-year study. 


\section{Sediment Pond Efficiency}

Sediment ponds, recognized as one of the most successful ways to reduce sediment inflow to receiving waterways, collect runoff from farmed fields and drains. If a pond is designed properly, the sands and larger silt particles tend to settle out, leaving only the finer sediment particles to be transported downstream. The ratio of the sediment outflow (discharge) from the ponds divided by the sediment inflow, times 100, is defined as the "trap efficiency," in percent.

There are several small sediment ponds in the study area, mostly above SLI Road in basins 59.6 and 60.7. Clean-out data on sediment ponds during the study period are not available. However, one large pond was sampled periodically at sites 19-22 (fig. 1) about 1/2 mile above the sampling station on Drain 60.7 and below SLI Road. The trap efficiency of this sediment pond is plotted in figure 4, and the sediment data are listed in table 12. The sediment concentrations of inflow sediment concentrations include some estimates to account for more than one source of water. Some water from site 20 tends to dilute the water entering the pond from site 19. Water flowing from site 20 came from an underground drain, which at times probably has some irrigation return flow fed directly into it. This effect is estimated for times when site 20 was not sampled.

The estimated average pond efficiency was 70 percent for the three irrigation seasons. The greatest efficiency during the irrigation season is about June to August (fig. 4). Trap efficiency tends to drop when sediment concentrations are lowest. However, the efficiency during the February 1980 storm was estimated to be 15 percent, a time when sediment concentrations were high. Most of the decrease in efficiency may be due to a decrease in retention time, caused by an increase in water discharge and silt and clay, and no storage. The pond was full of sediment by the end of the 1979 irrigation season. (The pond was cleaned prior to the 1980 irrigation season.)

The monthly hydrographs for all four drains (fig. 2) are similar; the hydrograph for Drain 60.7 does not reflect any major differences attributable to sediment pond efficiency. The trap efficiency may not be great enough to affect the overall sediment distribution, and sediment contribution coming in below the pond may also tend to mask out the impact of this sediment pond. If the pond were not there, then the sediment discharge of Drain 60.7 at site 2 would probably be greater, but by how much is not known. 


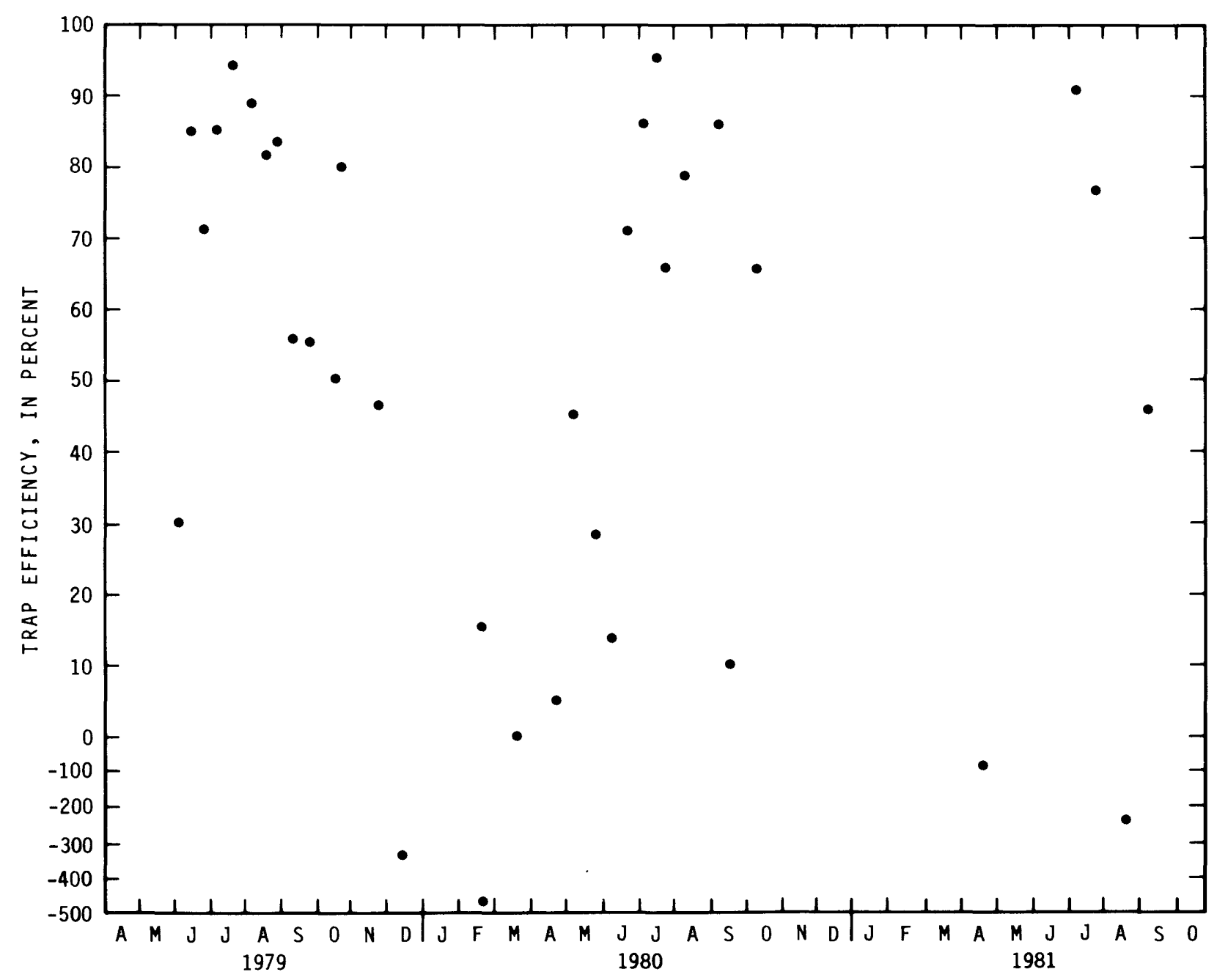

FIGURE 4.--Trap efficiency of sediment pond on Drain 60.7 , on the basis of data obtained at sites 19-22. 


\section{Size Analysis}

Twenty-eight sediment samples were collected in the four study drains for analysis of particle size - one from Drain 61.0, on February 20, 1980; four for Drain 60.7, from February 1980 to June 1981; eight for Drain 59.6, from June 1979 to July 1981; and 15 for Drain 59.4, from June 1979 to July 1981 (tables 9a-d; end of report). Samples from Drains 59.6 and 59.4 were about 75 percent silt, 20 percent clay, and 5 percent sand. Four samples from Drain 60.7 and one from Drain 61.0 were 50 percent clay, 45 percent silt, and 5 percent sand. Generally, Drains 59.6 and 59.4 had a preponderance of silt.

The sample from Drain 61.0, collected on February 20, 1980, had a sediment concentration of $1,000 \mathrm{mg} / \mathrm{L}$, an instantaneous sediment discharge of 6.2 tons/day. It was taken during the period of heaviest sediment transport in the drain. Because the sediment discharge from Drain 61.0 during the study was only 16.6 tons, the one sample is fairly representative of the particle size during the study. This sample, 50 percent clay, contrasts with samples taken in the other three drains for the same runoff period: 25, 16, and 41 percent clay in Drains 59.4, 59.6 , and 60.7 , respectively.

The particle size of the fluvial sediments reflects the loam and very fine sandy loam soils. Sand does not appear in large amounts in the soils, and therefore is not available in large amounts for transport by water. 


\section{ANALYSIS OF IMHOFF CONE DATA}

One purpose of this report is to discuss the relation between Imhoff Cone measurements and suspended sediment at the four daily-sampling sites (sites 1-4). Washington State University, Department of Agricultural Engineering, under the direction of Dr. Larry King, has taken the lead in relating Imhoff Cone measurements to suspended sediment, and those results to soil type. Preliminary data analyzed by Dr. King (written commun., 1982) indicate that the finer the soil particles, the poorer the correlation between Imhoff Cone (settleable solids) and suspended sediment (total solids). For example, Dr. King found that the correlation coefficient ranged from 0.97 for a sandy loam soil to 0.3 for a clay loam soil. Perhaps a longer settling time would result in a better correlation coefficient for clay-type soils.

There are problems in obtaining Imhoff Cone readings because of the subjective method used to determine the top level of settleable solids. Experience has shown that the solids settle out in the cone so the top layer is not always level, or normal with the cone axis. The sloping surface causes subjective interpretation that becomes increasingly significant with smaller total amounts of settleable solids. More accurate readings are obtained with larger amounts of settleable material.

Imhoff Cone analyses of samples from the four drains are listed in table 10 at the end of this report. Few samples were collected from Drain 61.0 because of the low sediment concentrations at this site. Also many Imhoff Cone samples were collected at all sites when no visible amount of settleable solids was observed, and these data are listed in table 10 as less than $0.1 \mathrm{ml} / \mathrm{L}$. When the amount of settleable solids was below 0.2 or $0.01 \mathrm{ml} / \mathrm{L}$, the readings were removed from data analysis because of the reason stated above.

Figures 5-8 show the plots of suspended sediment versus Imhoff Cone readings for each of the drain sites. No regression analysis was obtained for Drain 61.0 for the reasons stated above, but the data exceeding $0.01 \mathrm{ml} / \mathrm{L}$ are plotted in figure 5 .

The coefficient of determination, $r^{2}$ (correlation coefficient squared), is a relative measure of the accuracy of fit of the equation to the data, and is also a measure of the relative amount that the independent variable explains the regression population. The $\mathrm{r}^{2}$ value for Drains 60.7 and 59.4 was 0.81 , and for Drain 59.6 was 0.88 .

The standard error of the dependent variable (sediment concentration), another measure of the fit of the equation to the data, was quite high; for example, $1,070 \mathrm{mg} / \mathrm{L}$ for Drain $60.7,2,410 \mathrm{mg} / \mathrm{L}$ for Drain 59.6 , and $2,460 \mathrm{mg} / \mathrm{L}$ for Drain 59.4. The closest dashed line on figures 5-8 is the 95-percent confidence band about the mean of the regression on the dependent variable, suspended-sediment concentration. The outer dashed lines are the 95-percent confidence bands of the individual value predictions. 
The Imhoff Cone method of determining sediment transport and sediment yield for the sites studied could result in a large error. If the method were used for comparison of sediment transport between drainages, mean sediment concentrations would have to differ by several fold before a statistical difference could be established.

An analysis of covariance for the regressions for Drain $60.7,59.6$, and 59.4 indicated no statistical difference between regressions at the 95-percent confidence level for Drains 59.6 and 60.7 and Drains 59.4 and 60.7, but showed a difference between the regressions for Drains 59.6 and 59.4. 


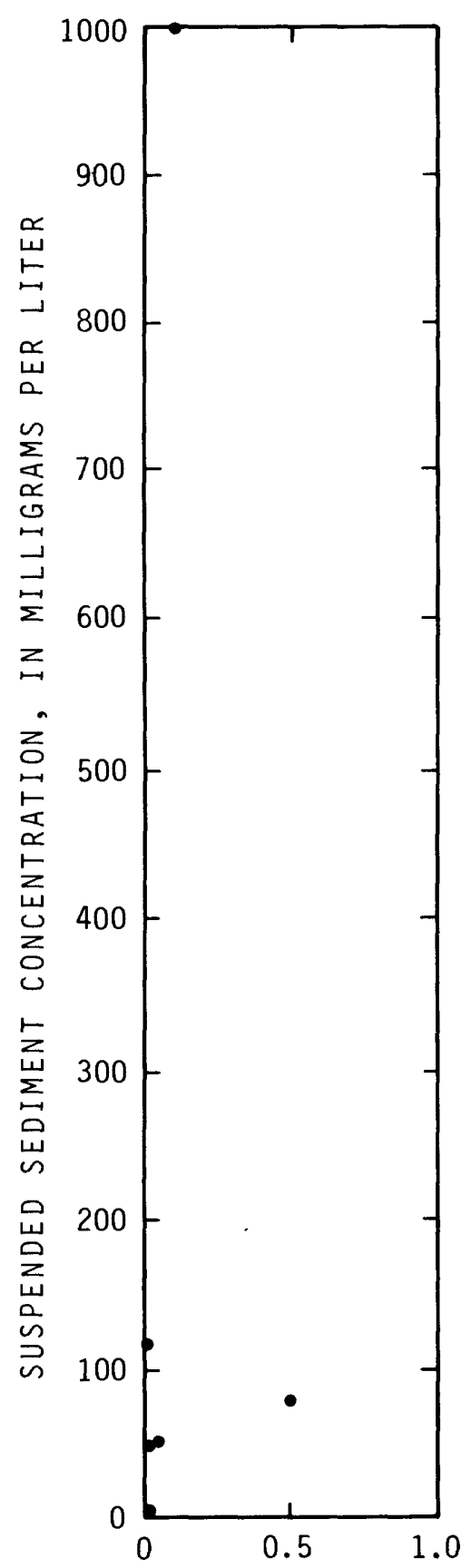

IMHOFF CONE SETTLEABLE SOLIDS PER 15 MINUTES, IN MILLILITERS PER LITER

FIGURE 5.--Relation of sediment concentration to Imhoff Cone settleable solids during the period April 1979 to October 1981 at site 1 (Drain 61.0, near Sunnyside). 


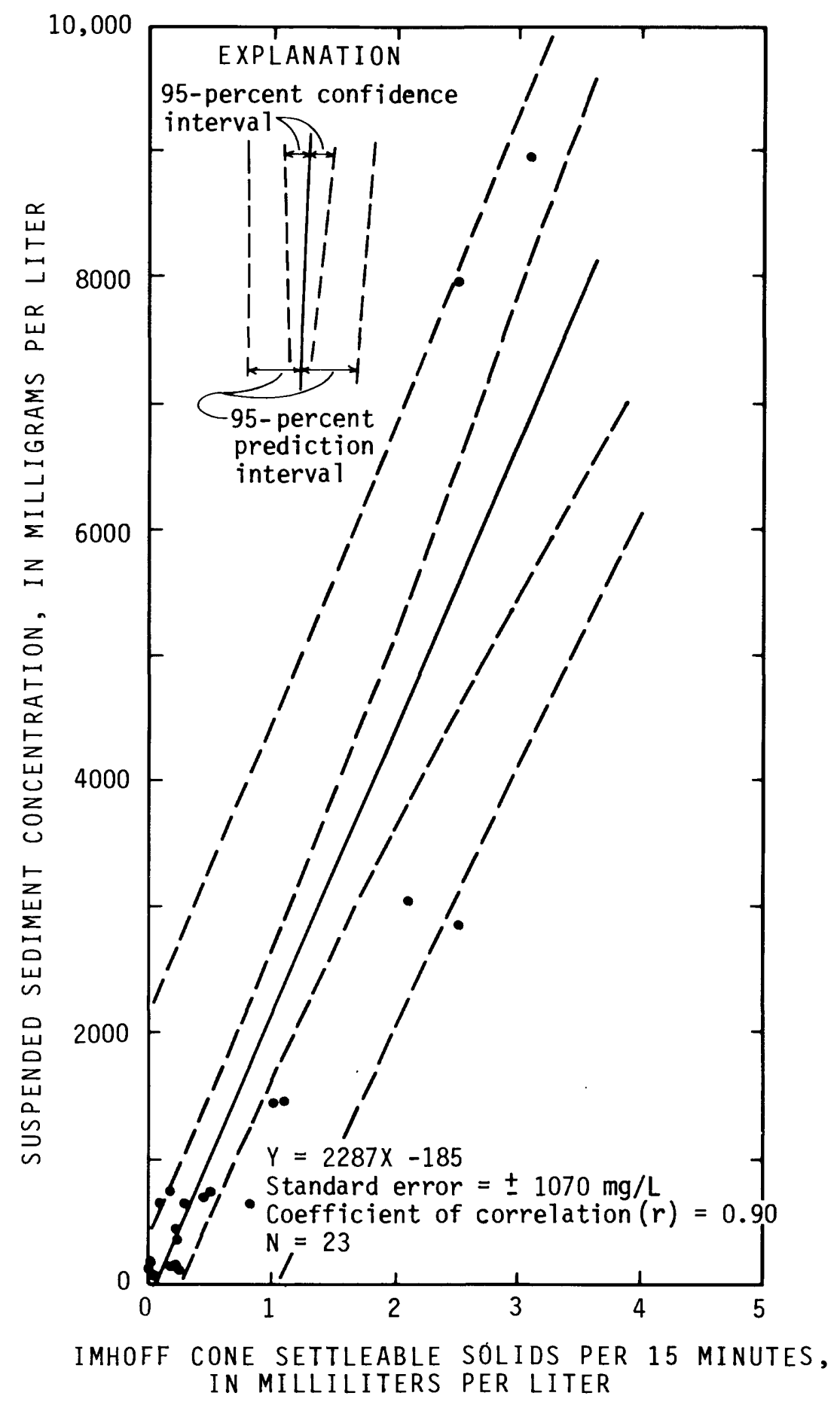

FIGURE 6.--Relation of sediment concentration to Imhoff Cone settleable solids during the period April 1979 to October 1981 at site 2 (Drain 60.7, near Sunnyside). 


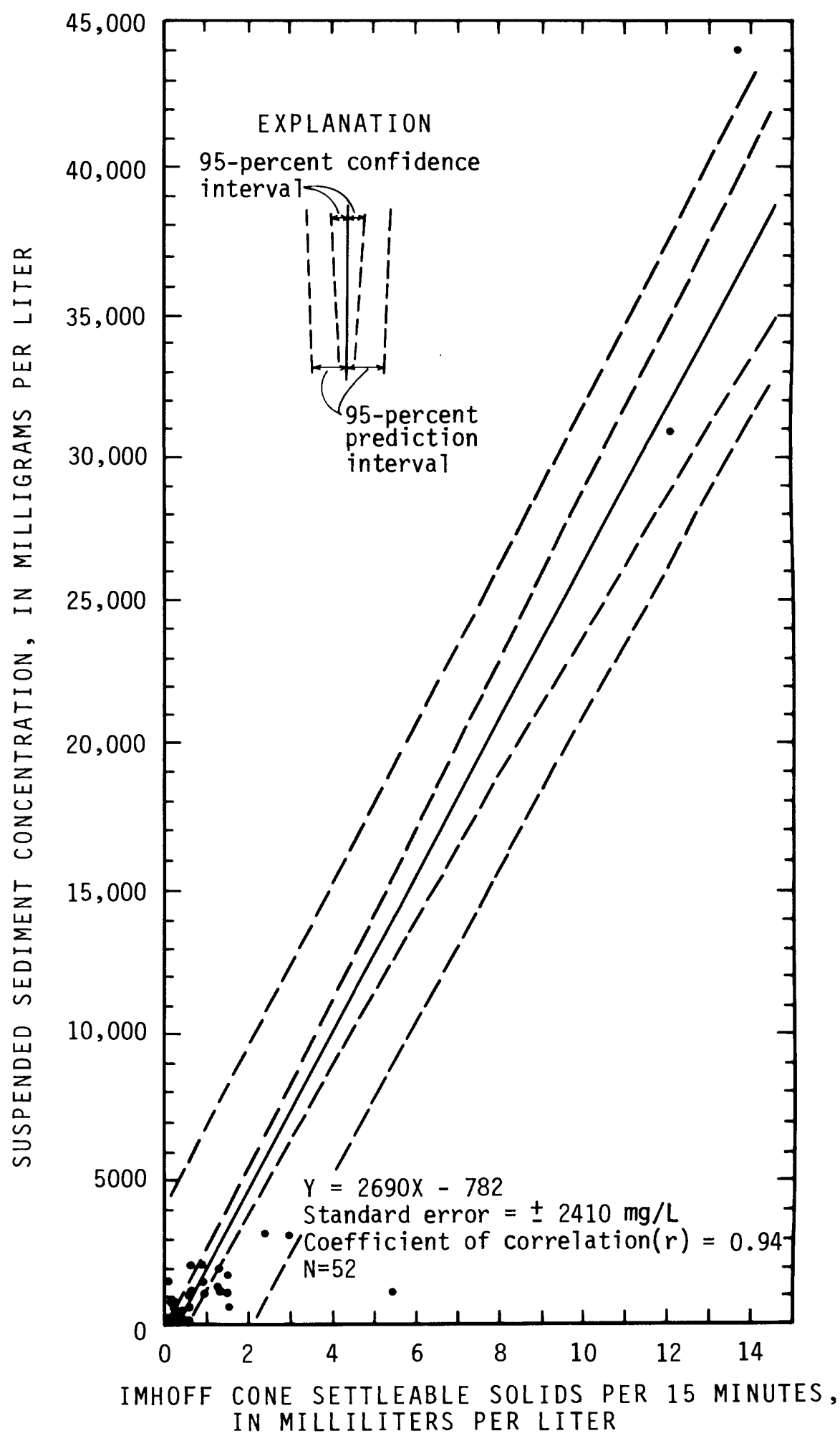

FIGURE 7.--Relation of sediment concentration to Imhoff Cone settleable solids during the period April 1979 to October 1981 at site 3 (Drain 59.6 below Drain 60.2, near Sunnyside). 


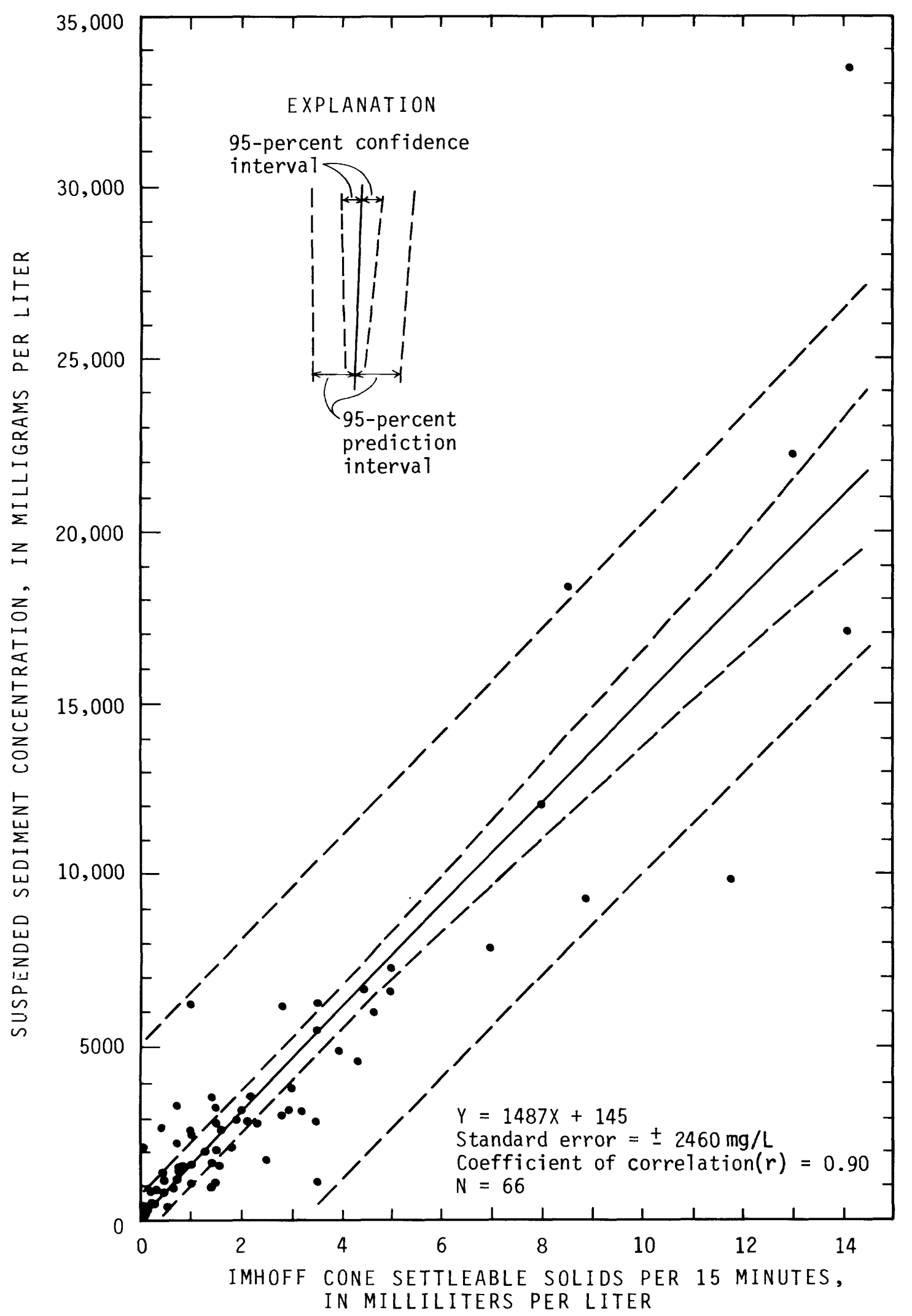

FIGURE 8.--Relation of sediment concentration to Imhoff Cone settleable solids during the period April 1979 to October 1981 at site 4 (Drain 59.4, near Sunnyside). 


\section{WATER TEMPERATURES}

Water temperatures were measured periodically in the drains and at the inflow daily station (site 7) to determine general temporal distributions and to provide an indication of the temperature differences between delivered water and the irrigation flows in the drains. Water temperatures were taken much less frequently during the nonirrigation periods than during the irrigation periods. Therefore, although the nonirrigation periodic data were used to help analyze the irrigation period by harmonic analysis, no median temperatures were computed for the nonirrigation season. Water temperatures were generally taken between 10:00 a.m. and 2:00 p.m. at all sites.

Water temperatures taken at the diversion on Roza Canal at mile 59.9 (site 7) were used to define temperatures for inflowing water. The 1979 irrigation season was divided into two periods, April 1 to September 7 and October 18 to November 6 , because there was no flow in the canal from September 8 to October 17 . No water temperatures were taken from October 18 to November 6, hence, seasonal temperatures for the 1979 irrigation season at site 7 can only be compared for April through August.

According to table 7, which summarizes the monthly mean temperatures generated by harmonic analysis, water temperatures at the diversion point on Roza Canal were warmer than those at the drain sites, with the exception of Drain 59.4, where the canal delivery water was cooler. In comparison with the canal diversion water, temperatures in Drain 61.0 and 59.6 averaged about $1.5^{\circ} \mathrm{C}$ cooler, Drain 60.7 about $0.5^{\circ} \mathrm{C}$ cooler, and Drain 59.4 about $1.5^{\circ} \mathrm{C}$ warmer.

Drain 61.0 had less flow than Drain 60.7 and Drain 59.6, yet water was about $1^{\circ} \mathrm{C}$ cooler than in Drain 60.7 and about $0.1^{\circ} \mathrm{C}$ cooler than in Drain 59.6. Drain 61.0 contains less sediment than the other drains, indicating that it probably receives less runoff from fields in comparison with "input" from ground water. Since one would expect ground-water seepage to be cooler than runoff from fields, this may account for the cooler temperature in Drain 61.0. Drain 59.4 is much warmer than the other drains because it receives runoff from fields in greater proportion to flows from ground water.

Water temperatures in the drains are generally warmer in June and July than the other months. Water temperatures at the 59.9 Roza Canal diversion are warmest during July and August. 
TABLE 7.--Median of mean water temperatures at five sites in the DID-18 basin of the Sulphur Creek basin for the 1979, 1980, and 1981 irrigation seasons, by harmonic analysis (confidence interval is at the 90 -percent level)

\begin{tabular}{|c|c|c|c|c|c|c|c|c|c|c|}
\hline \multirow{2}{*}{ Site } & \multirow{2}{*}{ Location } & \multirow{2}{*}{$\begin{array}{l}\text { Irriga- } \\
\text { tion } \\
\text { season }\end{array}$} & \multicolumn{7}{|c|}{ Median monthly temperature $(O C)$} & \multirow{2}{*}{$\begin{array}{l}\text { Mean irriga- } \\
\text { tion season } \\
\text { temperature } \\
(\mathrm{OC})\end{array}$} \\
\hline & & & Apr. & May & June & July & Aug. & Sept. & oct. & \\
\hline 1 & $\begin{array}{l}\text { Drain } 61.0 \text { below } \\
\text { drain } 61.4\end{array}$ & $\begin{array}{l}1979 \\
1980 \\
1981\end{array}$ & $\begin{array}{l}12.6 \\
13.6 \\
12.2\end{array}$ & $\begin{array}{l}15.2 \\
14.9 \\
14.5\end{array}$ & $\begin{array}{l}16.8 \\
15.5 \\
16.1\end{array}$ & $\begin{array}{l}17.0 \\
15.2 \\
16.7\end{array}$ & $\begin{array}{l}15.7 \\
14.2 \\
16.0\end{array}$ & $\begin{array}{l}13.3 \\
12.7 \\
14.2\end{array}$ & $\begin{array}{l}10.5 \\
11.1 \\
11.9\end{array}$ & $\begin{array}{l}14.3+0.5 \\
14.0 \mp .3 \\
14.6 \pm .5\end{array}$ \\
\hline 2 & Drain 60.7 & $\begin{array}{l}1979 \\
1980 \\
1981\end{array}$ & $\begin{array}{l}14.3 \\
14.3 \\
13.4\end{array}$ & $\begin{array}{l}15.7 \\
15.6 \\
15.3\end{array}$ & $\begin{array}{l}16.7 \\
16.3 \\
16.7\end{array}$ & $\begin{array}{l}17.0 \\
16.3 \\
17.1\end{array}$ & $\begin{array}{l}16.6 \\
15.6 \\
16.5\end{array}$ & $\begin{array}{l}15.5 \\
14.4 \\
15.1\end{array}$ & $\begin{array}{l}14.0 \\
13.0 \\
13.1\end{array}$ & $\begin{array}{l}15.6 \pm .3 \\
15.2 \pm .2 \\
15.4 \pm .4\end{array}$ \\
\hline 3 & $\begin{array}{l}\text { Drain } 59.6 \text { below } \\
\text { Drain } 60.0\end{array}$ & $\begin{array}{l}1979 \\
1980 \\
1981\end{array}$ & $\begin{array}{l}13.2 \\
13.6 \\
13.2\end{array}$ & $\begin{array}{l}15.5 \\
14.9 \\
15.0\end{array}$ & $\begin{array}{l}16.9 \\
15.6 \\
16.1\end{array}$ & $\begin{array}{l}17.0 \\
15.3 \\
16.2\end{array}$ & $\begin{array}{l}15.8 \\
14.3 \\
15.2\end{array}$ & $\begin{array}{l}13.6 \\
12.8 \\
13.5\end{array}$ & $\begin{array}{l}11.0 \\
11.1 \\
11.4\end{array}$ & $\begin{array}{l}14.6 \pm .4 \\
14.1 \pm .3 \\
14.5 \pm .3\end{array}$ \\
\hline 4 & $\begin{array}{l}\text { Drain } 59.4 \text { above } \\
\text { Drain } 59.6\end{array}$ & $\begin{array}{l}1979 \\
1980 \\
1981\end{array}$ & $\begin{array}{l}16.1 \\
15.8 \\
15.2\end{array}$ & $\begin{array}{l}18.8 \\
17.5 \\
18.5\end{array}$ & $\begin{array}{l}20.1 \\
18.1 \\
20.5\end{array}$ & $\begin{array}{l}19.6 \\
17.4 \\
20.7\end{array}$ & $\begin{array}{l}17.5 \\
15.6 \\
18.9\end{array}$ & $\begin{array}{l}14.4 \\
13.1 \\
15.7\end{array}$ & $\begin{array}{l}11.1 \\
10.7 \\
11.9\end{array}$ & $\begin{array}{l}16.6 \pm .6 \\
15.8 \mp .5 \\
17.6 \pm .5\end{array}$ \\
\hline 7 & $\begin{array}{c}\text { Roza Canal at } \\
\text { mile } 59.9\end{array}$ & $\begin{array}{l}1979 \\
1980 \\
1981\end{array}$ & $\begin{array}{l}12.4 \\
11.1 \\
10.1\end{array}$ & $\begin{array}{l}14.7 \\
14.3 \\
14.2\end{array}$ & $\begin{array}{l}17.4 \\
17.0 \\
17.4\end{array}$ & $\begin{array}{l}19.8 \\
18.3 \\
18.9\end{array}$ & $\begin{array}{l}21.2 \\
17.9 \\
18.2\end{array}$ & $\begin{array}{c}\star \\
15.8 \\
15.6\end{array}$ & $\begin{array}{c}\star \\
13.2 \\
12.6\end{array}$ & $\begin{array}{r}* \star 17.2 \pm .3 \\
15.6 \pm .2 \\
15.4 \pm .3\end{array}$ \\
\hline
\end{tabular}

* No flow September 8 to October 17.

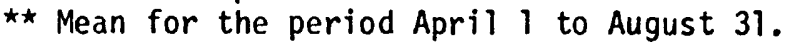


A site sampled by Boucher and Fretwell (1982) during the 1976 irrigation season (site C, Roza Canal below Wasteway No. 5, fig. 1) had water temperatures much cooler than those observed during the present study at the diversion at 59.9 ( $1 / 2$ mile downstream of the 1976 site). The median water temperature at the site for the 1976 irrigation season was $14.4^{\circ} \mathrm{C}$; the median temperatures for the 1980 and 1981 irrigation seasons, at the diversion at mile 59.9 , were $15.6^{\circ} \mathrm{C}$ and $15.4^{\circ} \mathrm{C}$, respectively. One explanation for this is that in 1976 temperatures were measured at midstream, while in 1980 and 1981 at diversion 59.9 they were taken next to the canal bank, where slower velocities and shallower depths result in warmer water.

The median water temperatures at Roza Canal below Wasteway No. 5 (site C), for the 1976 irrigation season were as follows:

\begin{tabular}{ccccccccc} 
& \multicolumn{8}{c}{1976 irrigation season } \\
& Apr. & May & June & July & Aug. & Sept. & Oct. & Mean \\
Temp oC & 9.0 & 12.7 & 15.5 & 17.4 & 17.5 & 15.8 & 12.8 & 14.4
\end{tabular}




\section{SUMMARY}

Sediment discharges from the four study basins could not be correlated with changes in Best Management Practices for two reasons: (1) the Washington State Department of Ecology found no statistical difference in the Imhoff Cone readings and comparison of irrigation methods and Best Management Practices for the three irrigation seasons, and (2) changes in Best Management Practices were not implemented on a large enough scale to be detected at the main drain sampling stations.

The potential for erosion in the four basins is similar, but Drain 59.4 (site 4) had the highest average sediment yield for the three irrigation seasons--1.5 tons/acre, as compared to 1.0 ton/acre for Drain 59.6, 0.6 ton/acre for Drain 60.7, and -0.16 ton/acre for Drain 61.0. As the percentage of row crops increased, the sediment yields tended to become greater, but a cause-effect relationship has not been established. Discharge-weighted mean suspended-sediment concentration during the three irrigation seasons was also much higher in Drain 59.4, averaging $2,460 \mathrm{mg} / \mathrm{L}$, as compared with $440 \mathrm{mg} / \mathrm{L}$ for Drain $59.6,290 \mathrm{mg} / \mathrm{L}$ for Drain 60.7 , and $12 \mathrm{mg} / \mathrm{L}$ for Drain 61.0 .

Sediment yield increased significantly for Drain 60.7 from the 1979 irrigation season to the 1981 irrigation season. For Drain 59.6, the 1980 and 1981 sediment yields were significantly greater than the 1979 sediment yield. For Drain 59.4, the 1979 and 1981 sediment yields were not significantly different, but yields for both years were significantly greater than for 1980 , averaging 2.0 tons/acre compared with 0.64 ton/acre for 1980 .

Drain 61.0 acts as a sink for sediment, even though slopes there are similar to or even slightly greater (2-3 percent) than in the other drains. A difference in management practices in Drain 61.0 is assumed to be the cause. Drain 61.0 also had a high proportion of sprinklers. Even during the February 1980 storm, Drain 61.0 acted as a sink for sediment: 48 tons, or 3.5 times more sediment, flowed in from the portion of the drainage north of Roza Canal than was transported out of the basin at the monitoring point. This indicates deposition along the drainage way that could be available for transport at a later time.

The one storm, February 16-20, 1980, contributed 51 percent of the sediment discharge from Drain 61.0, 11 percent from Drain 60.7, 17 percent from Drain 59.6, and 14 percent from Drain 59.4. This illustrates the importance of sampling during storms and during the nonirrigation season to obtain the annual sediment discharge. A sediment pond half a mile above the sampling site for Drain 60.7 probably had some effect in reducing the sediment transport from this basin, since the pond trapped some sediment. The trap efficiency averaged about $\mathbf{7 0}$ percent in runoff periods during the three irrigation seasons. The pond was only about 15 percent efficient during the one storm runoff period in February 1980. The effect of other sediment ponds in the project area was not studied. 
There was little or no impact from ashfall from the eruption of Mount St. Helens on May 18, 1980. Only assumed minor increases in sediment concentrations occurred at the inflow monitoring point and the outflow point on Drain 61.0.

Size analysis of sediments shows a preponderance of silts and clays indicative of the silt-loam soils in the basin.

Comparison of Imhoff Cone data with suspended-sediment data resulted in correlation coefficients for Drains 60.7, 59.6, and 59.4 (sites 2, 3, and 4) of 0.90, 0.94 , and 0.90 , respectively. However, the standard error in sediment concentration for these drains was $1,070,2,410$, and $2,460 \mathrm{mg} / \mathrm{L}$, respectively. An analysis of covariance indicates that at the 95-percent confidence level there was no statistical difference in the regressions between Drains 59.6 and 60.7 and between Drains 59.4 and 60.7, but there was a difference between Drains 59.6 and 59.4.

The use of Imhoff Cone suspended-sediment concentration relation to determine sediment yields and discharges that are then compared between years would result in large error, since the prediction limits are very large. Imhoff Cone suspended-sediment concentration relation becomes less accurate for sediments having a high clay content. No Imhoff Cone relation was developed for Drain 61.0 because of low sediment concentrations; only a few readings were large enough to be reported.

Median irrigation season water temperatures determined by harmonic analysis in Drains 61.0 (site 1) and 59.6 (site 3 ) were about $1.5^{\circ} \mathrm{C}$ cooler than in the diversion water from Roza Canal at mile 59.9 (site 7). Drain 60.7 (site 2) median water temperatures were about $0.5^{\circ} \mathrm{C}$ cooler, but in Drain 59.4 (site 4) they were about $1.5^{\circ} \mathrm{C}$ warmer. The warmer water in Drain 59.4 was attributed to the lower base flows and higher irrigation runoff. The average median water temperatures in the drains for the 1979, 1980, and 1981 irrigation seasons for the drains were $14.3^{\circ} \mathrm{C}$ for Drain $61.0,15.4^{\circ} \mathrm{C}$ for Drain $60.7,14.4^{\circ} \mathrm{C}$ for Drain 59.6, and $16.6^{\circ} \mathrm{C}$ for Drain 59.4. 


\section{SELECTED REFERENCES}

Arkin, H., and Colton R. R., 1970, Statistical methods, College outline series: New York, Barnes and Noble, Inc., p. 153.

Baird, D. C., 1962, Experimentations: An introduction to measurement theory and experiment design: New Jersy, Prentice Ha11, Inc., 198 p.

Boucher, P. R. and Fretwe11, M.0., 1982, Irrigation-water quality in the Sulphur Creek basin, Yakima and Benton Counties, Washington April 1976 through March 1977: U.S. Geological Survey Open-File Report $81-1008,68 \mathrm{p}$.

Buchanan, T. J., and Somers, W. P., 1969, Discharge measurements at gaging stations: U.S. Geological Survey Techniques of Water Resources Investigations, Book 3, Chapter A8, 65 p.

Carter, R. W., and Davidian, Jacob, 1968, Genera1 procedures for gaging streams: U.S. Geological Survey Techniques of Water Resources Investigations, Book 3, Chapter A6, $13 \mathrm{p}$.

$\mathrm{CH}_{2} \mathrm{M} \mathrm{Hill,} \mathrm{1975,} \mathrm{Characterization} \mathrm{of} \mathrm{present} \mathrm{water-quality} \mathrm{conditions}$ in the Yakima basin: Washington Department of Ecology Technical Bulletin $10,47 p$.

----1977, A status report on water-quality investigations, Yakima River basin, Washington: Bellevue, Washington, $\mathrm{CH}_{2} \mathrm{M} \mathrm{Hill}, 111 \mathrm{p}$.

Collings, M. R., 1969, Temperature analysis of a stream: U.S. Geological Survey Professional Paper 650-B, p. B174-B179.

Guy, H. P., 1969, Laboratory theory and methods for sediment analysis: U.S. Geological Survey Techniques of Water-Resources Investigations, Book 5, Chapter $\mathrm{C} 1,58 \mathrm{p}$.

Guy, H. P., and Norman, V. W., 1970, Field methods for measurements of fluvial sediment: U.S. Geological Survey Techniques of Water-Resources Investigations, Book 3, Chapter C2, 59 p.

Nelson, L.M., 1979, Sediment transport by irrigation return flows in the Lower Yakima River basin, Washington, 1975 and 1976 irrigation seasons: U.S. Geological Survey Open-File Report 78-946, 76 p.

Riggs, H. C., 1968, Some statistical tools in hydrology: U.S. Geological Survey Techniques of Water-Resources Investigations, Book 4, Chapter A1, 39 p. 
Sarna-Wojcicki, A.M., Shipley, Susan, Waitt, R.B., Jr., Dzurisin, Danie1 and Wood, S. H., 1981, Areal distribution, thickness, mass, volume, and grain size of air-fall ash from the six major eruptions of 1980: in Lipman, P. W., and Mullineaux, D. R., eds., The 1980 Eruptions of Mount St. Helens, Washington: U.S. Geological Survey Professional Paper 1250, 844 p.

Steele, T. D., 1974, Harmonic analysis of stream temperature: U.S. Geological Survey Computer Contribution, December 1973, 246 p.: available only from the U.S. Department of Commerce, National Technical Information Service, Springfield, Virginia 22151, as report $\mathrm{PB}--23--016 / \mathrm{AS}$.

U.S. Geological Survey, 1979, 1980, 1981, Water resources data for Washington, part 2, 1980, 1981, 1982 .

U.S. National 0ceanic and Atmospheric Administration (formerly U.S. Weather Bureau), 1979, 1980, 1981 Climatological data, Washington, Annual Summary 1979, 1980, 1981: vol. 83, 84, 85, no. 13.

U.S. Soil Conservation Service, General soil map, Yakima County Area, March 1970.

Ward, J. C., 1963, Annual variation of stream water temperature: American Society of Civil Engineers Journal, vol. 89, no. SA6, p. 1-16.

Wittenberg, L.A., and McKenzie, S.W., 1980, Water quality of Bear Creek basin, Jackson County, Oregon: U.S. Geological Survey Open-File Report 80-158, 118 p. 


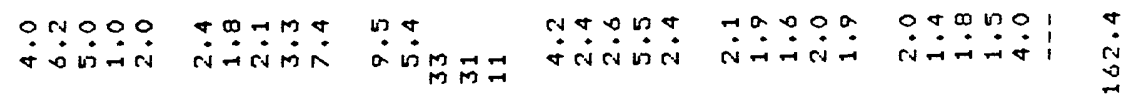

๙ิ

㑕责

岁

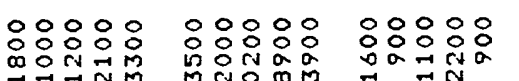

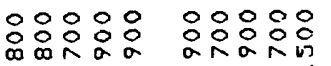

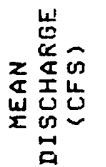

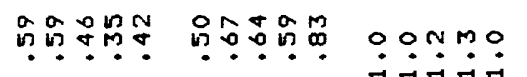

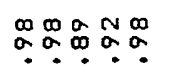

$\operatorname{Lin} 2$

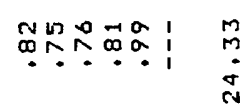

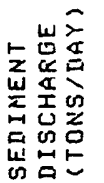

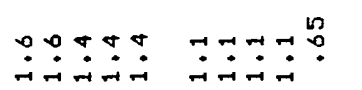

mam

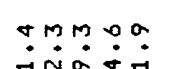

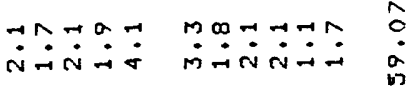

命

究总

<

$\therefore: \div: 0$

$\therefore: \therefore: \circ$

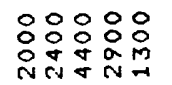

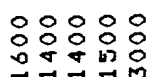

으유:

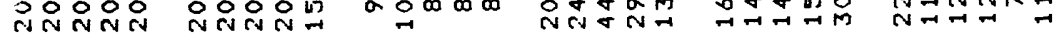

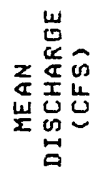

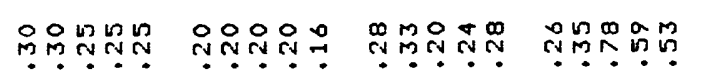

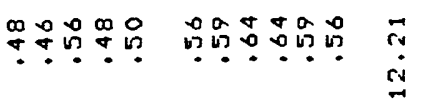

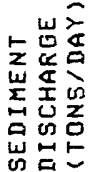

$0.95 ?$

ก:⿱一⿻上丨)

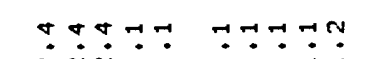

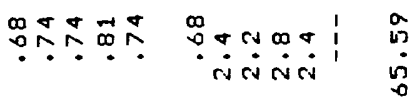

$\stackrel{9}{\stackrel{1}{\sigma}}$

热总

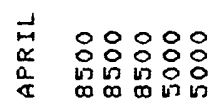

部品品品

염융유

$\therefore: \therefore::$

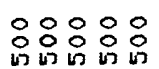

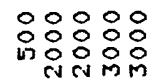

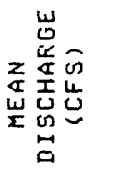

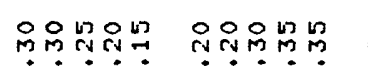

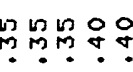

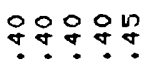

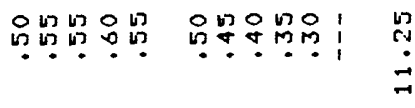

衣

tamen

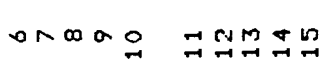

노ำㅇำ

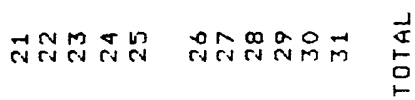




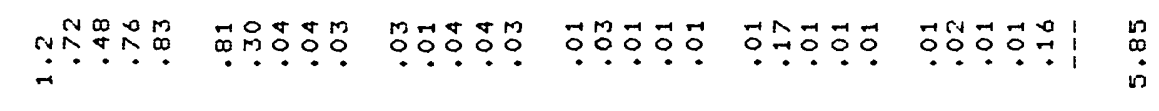
$\stackrel{\circ}{\check{\sigma}}$

象

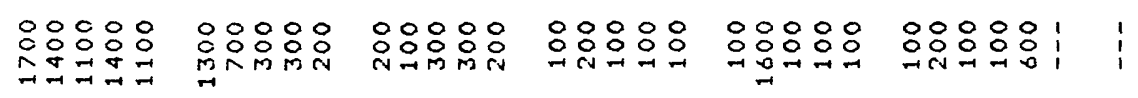

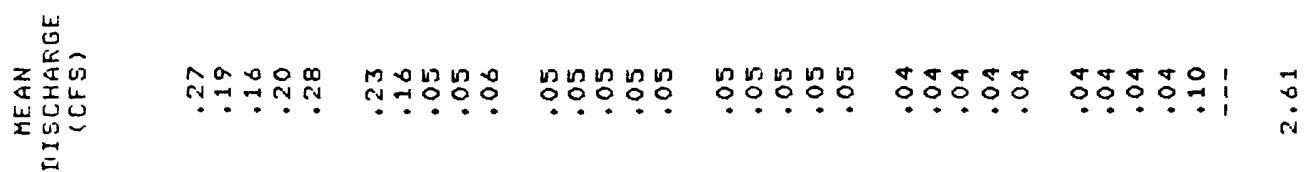

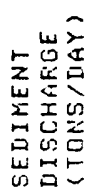

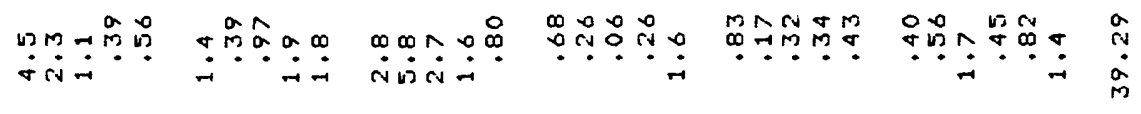

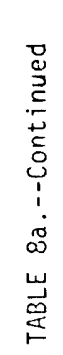

$\stackrel{\text { on }}{\frac{1}{m}}$

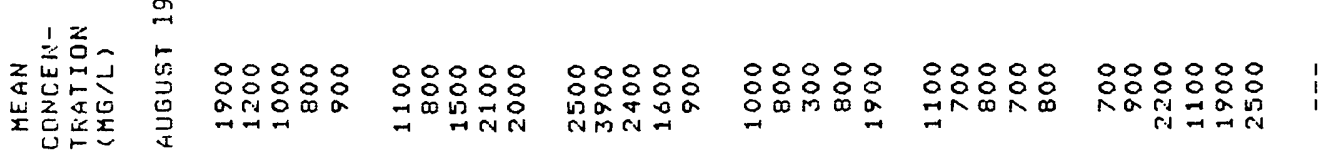

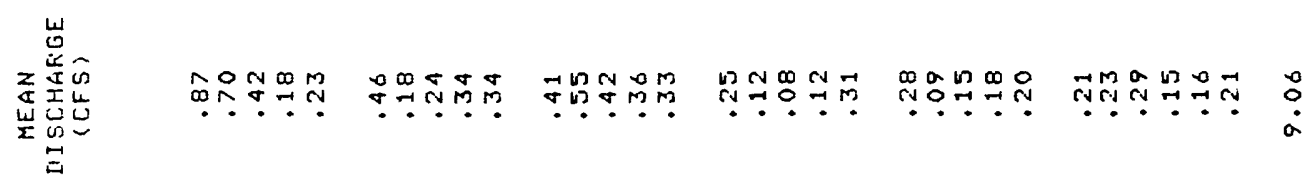

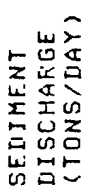

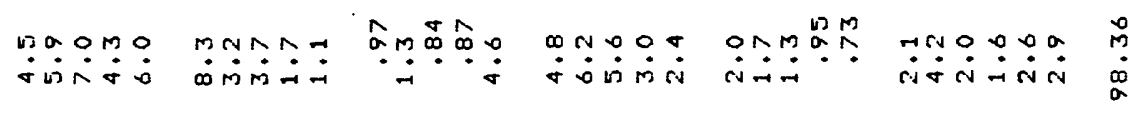

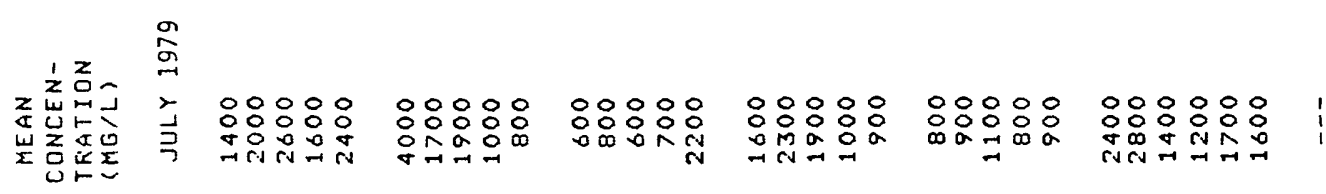

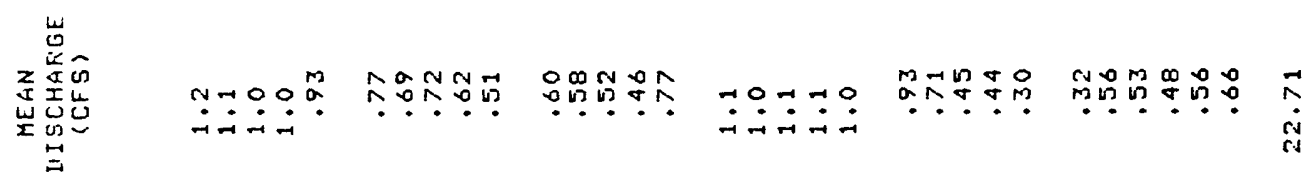

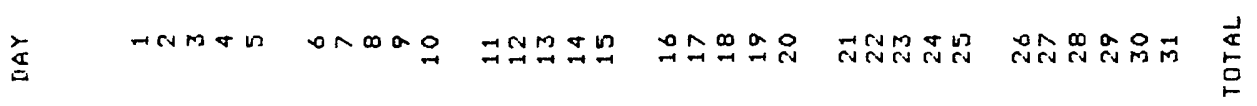




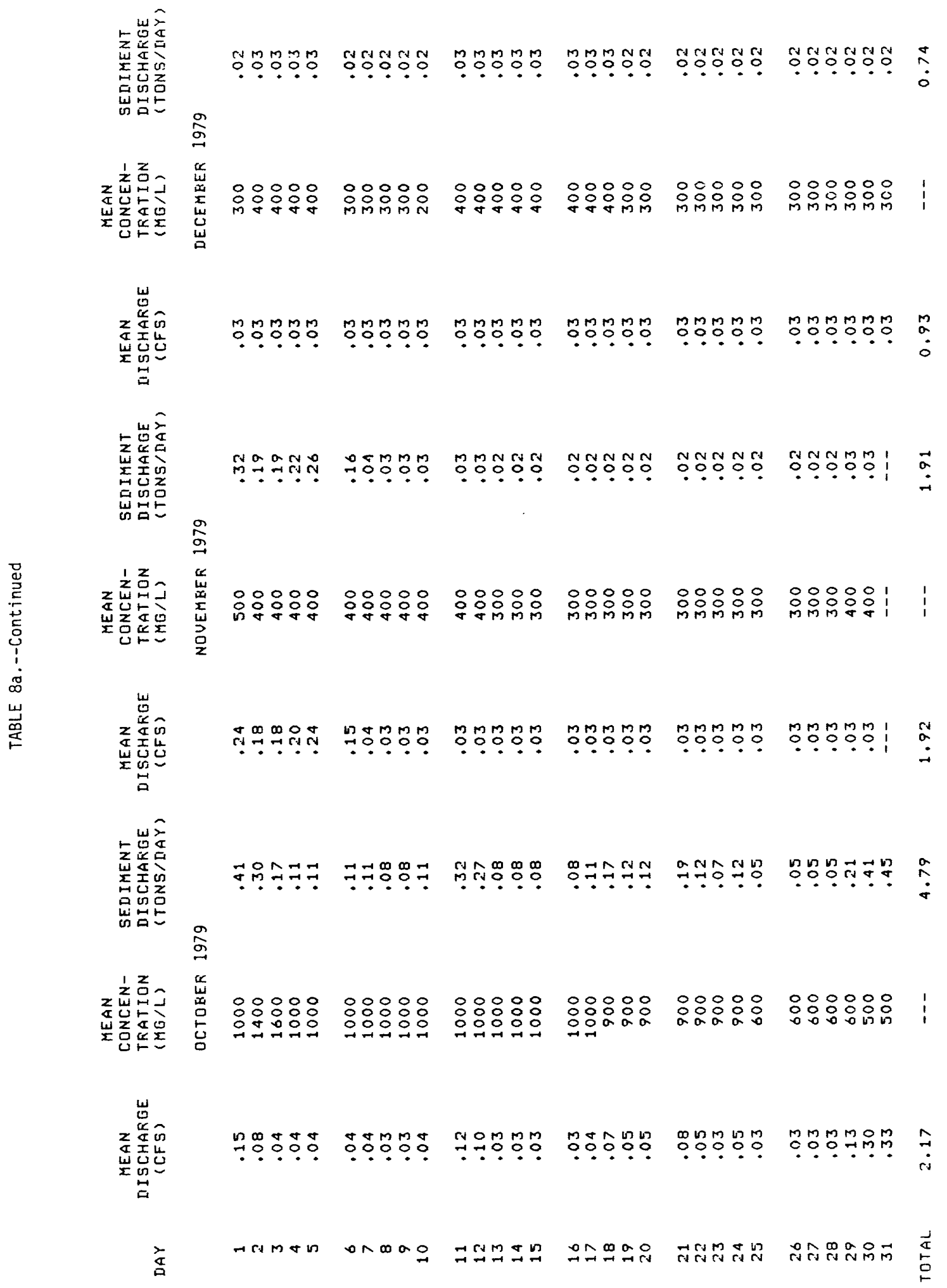




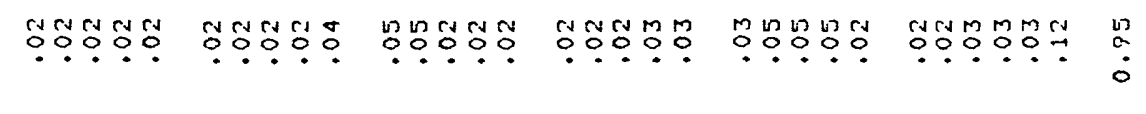

$\stackrel{\infty}{9}$

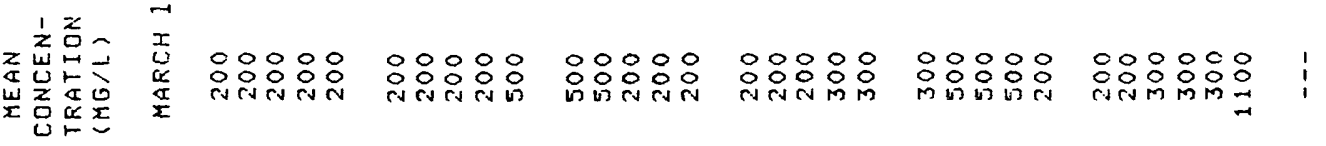

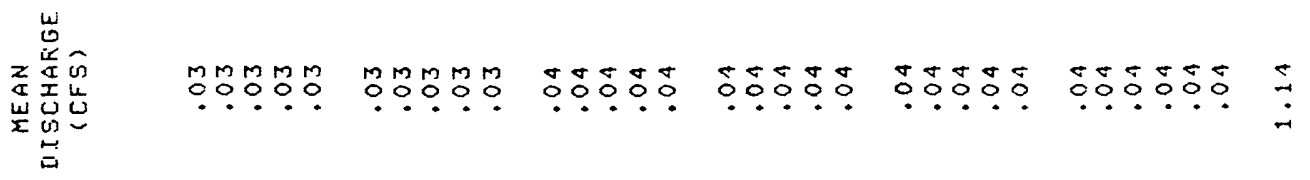

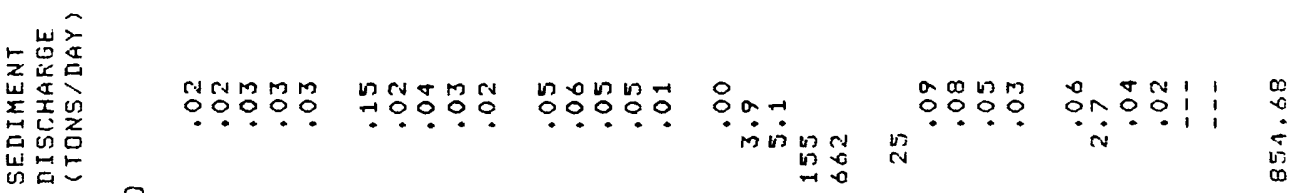

:

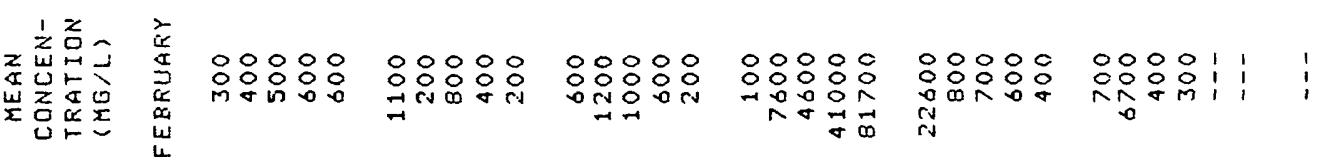

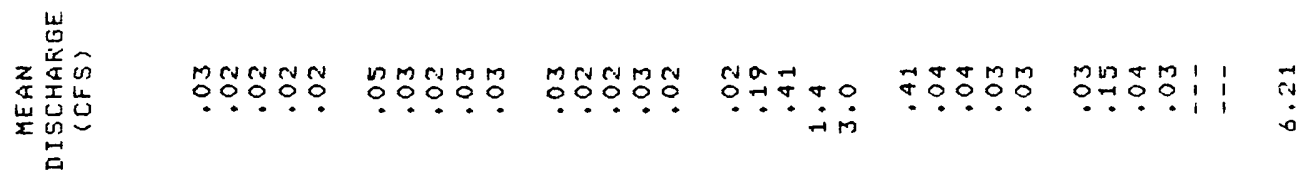

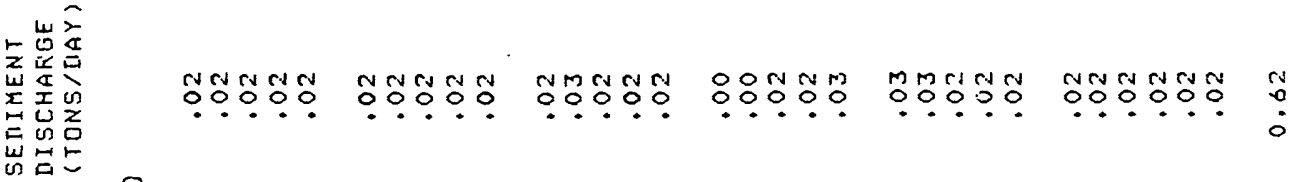

$\stackrel{9}{\infty}$

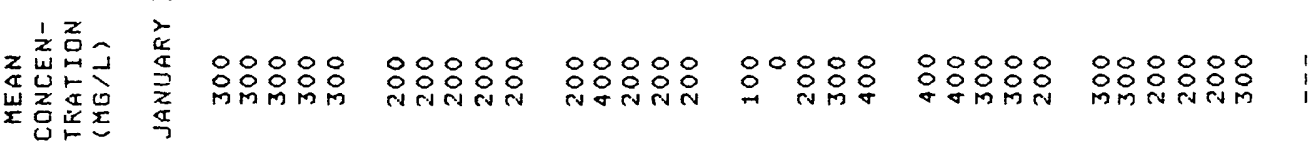

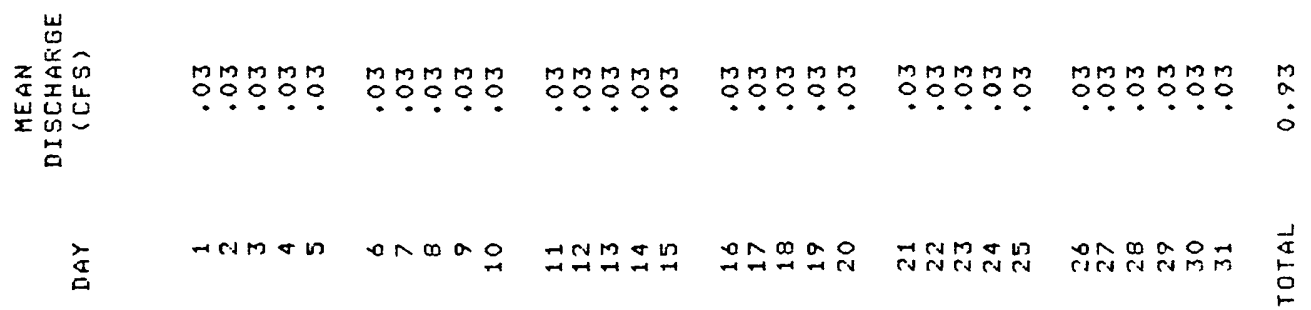




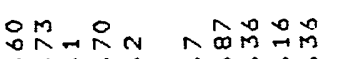

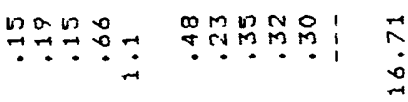

:

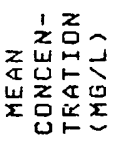

$\stackrel{8}{\stackrel{8}{-}}$ 
点总畜

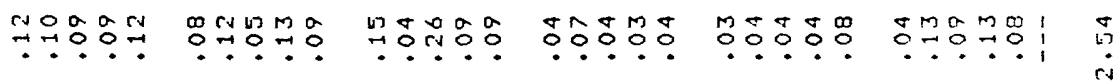

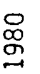

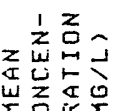

焉

연유

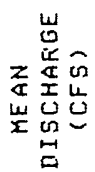

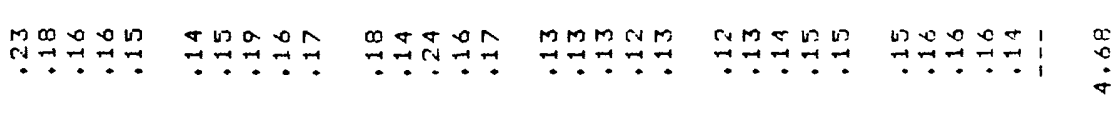

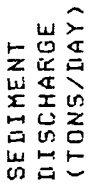

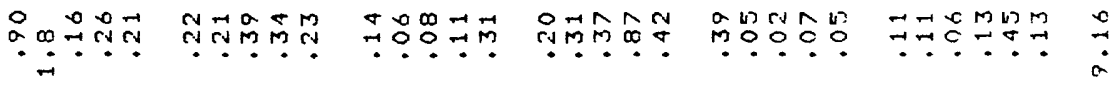

心

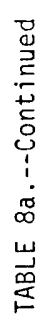

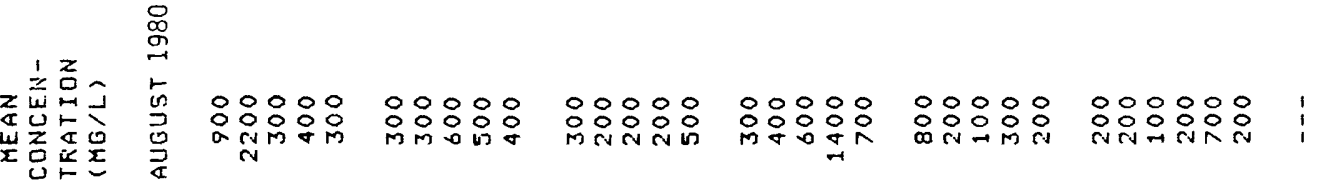

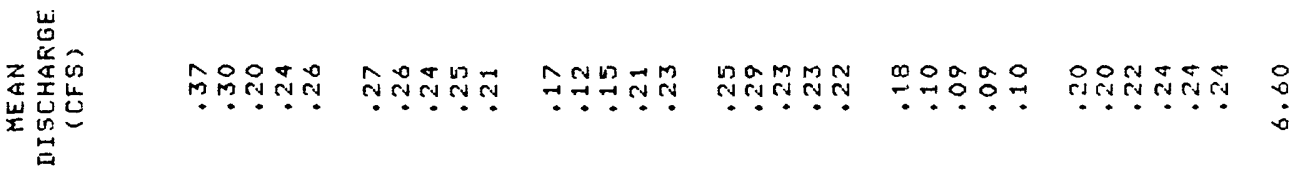

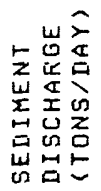

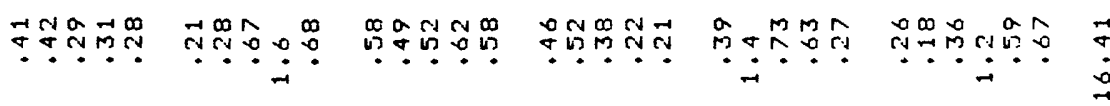

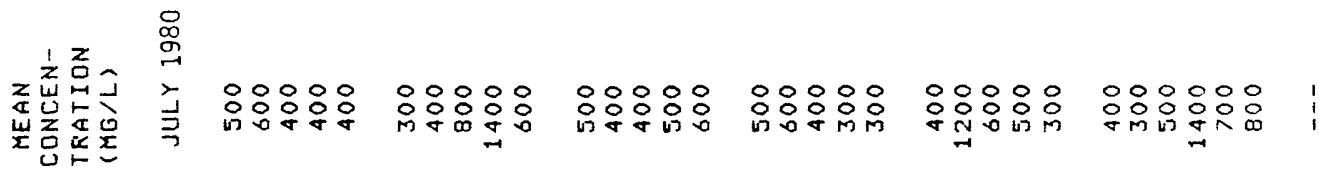

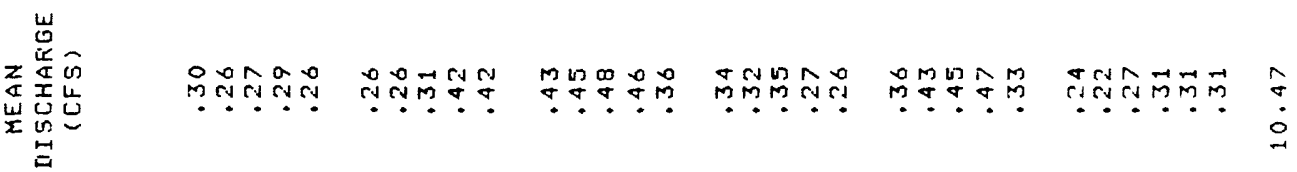

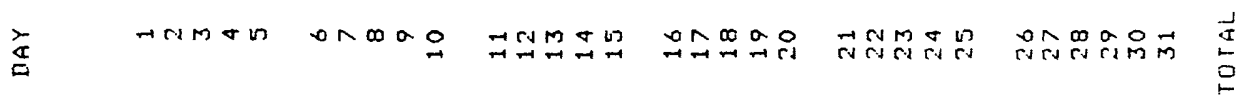




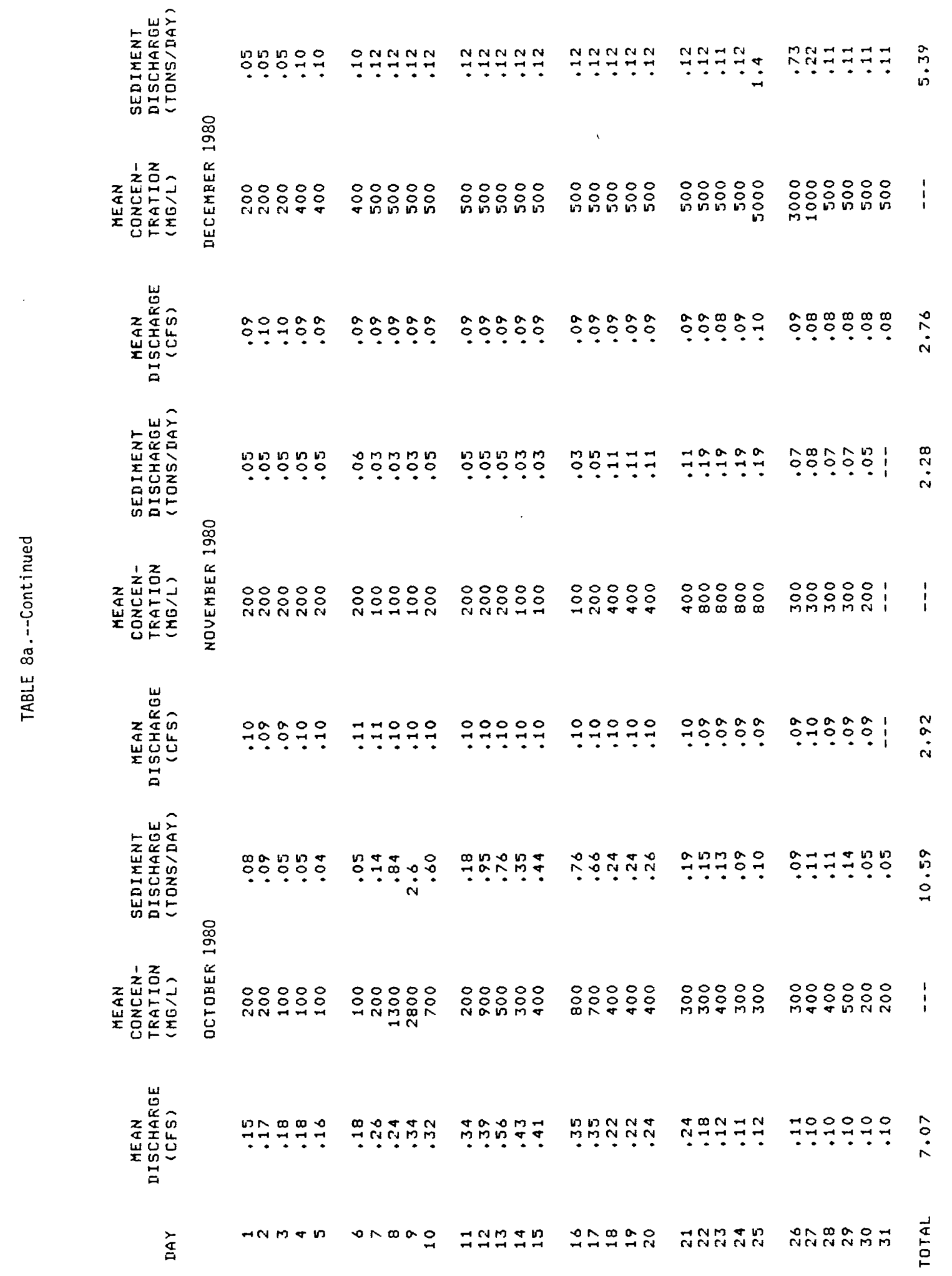




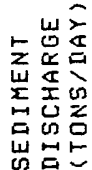

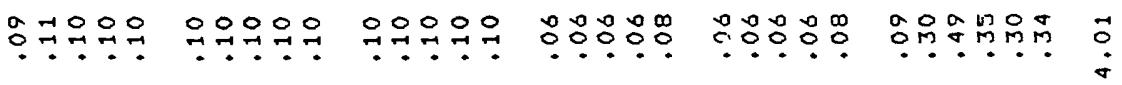

$\stackrel{\vec{g}}{\stackrel{్}{\sigma}}$

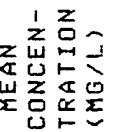

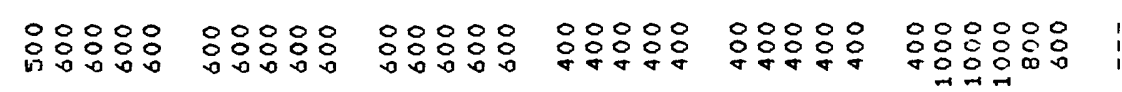

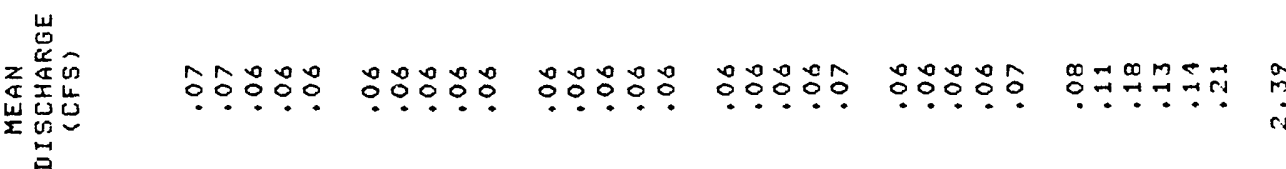

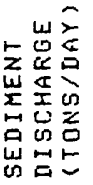

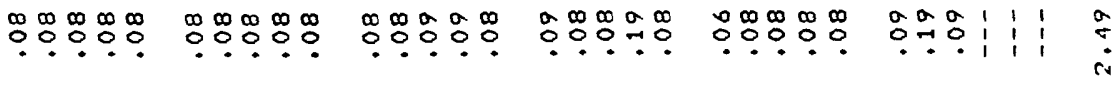

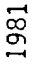

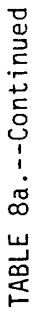

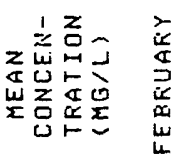

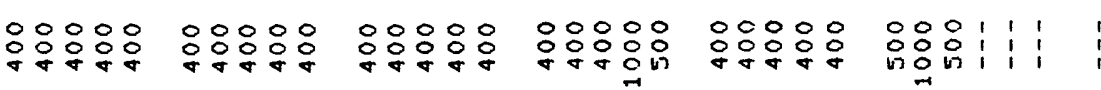

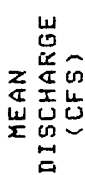

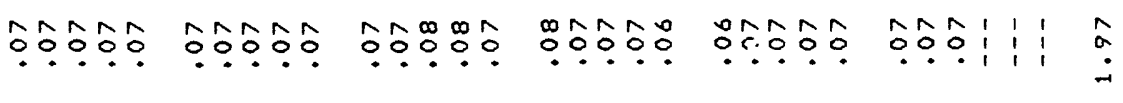

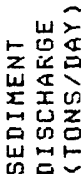

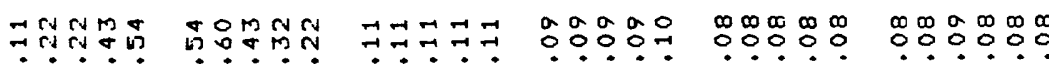

$\vec{\infty}$

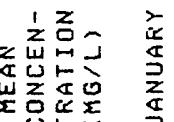

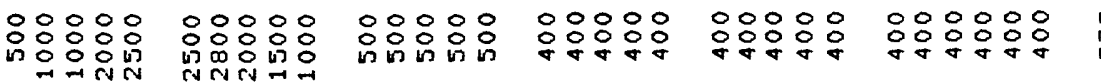

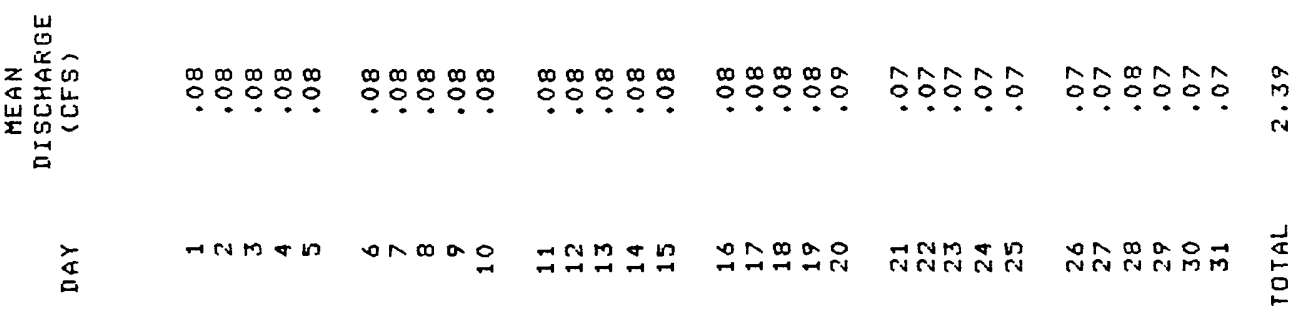




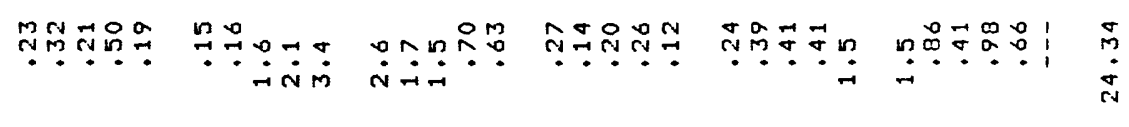

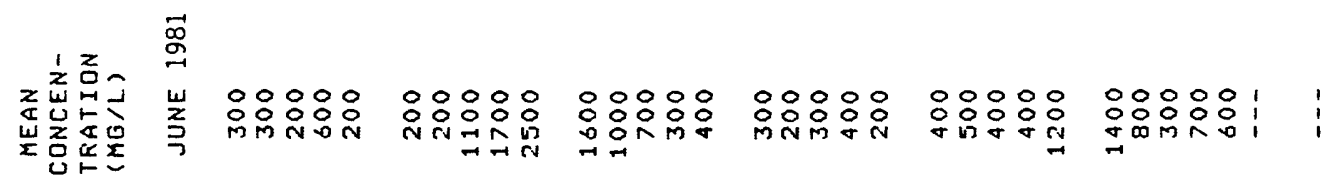

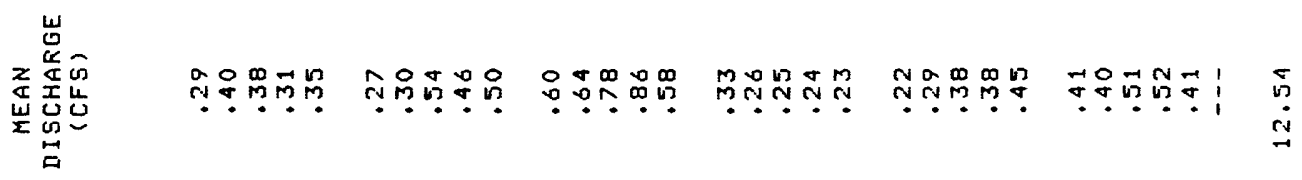

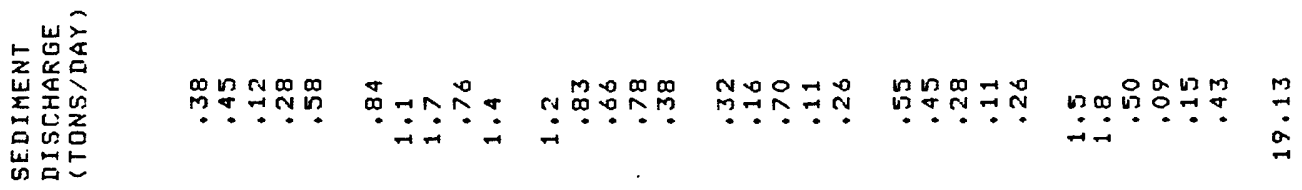

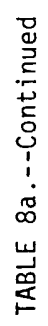

$\vec{\circ}$

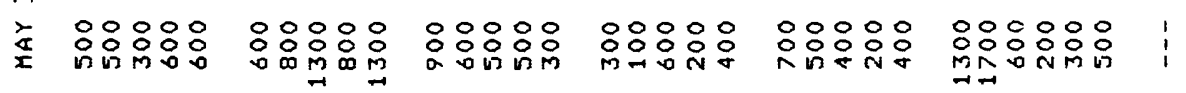

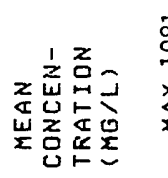

崖

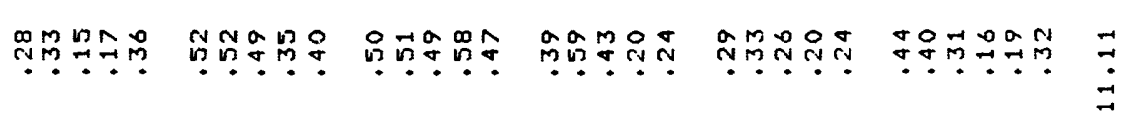

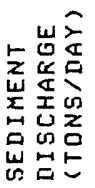

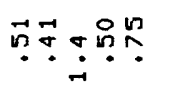

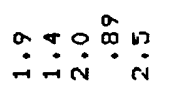

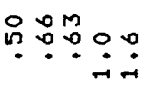

$\underset{\text { ind }}{2}$

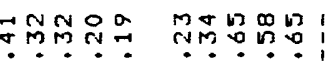

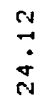

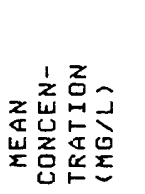

$\stackrel{\overrightarrow{0}}{\stackrel{7}{7}}$

응요

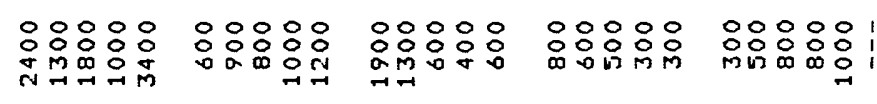

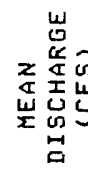

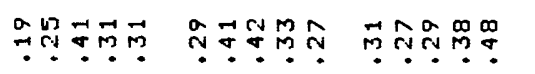

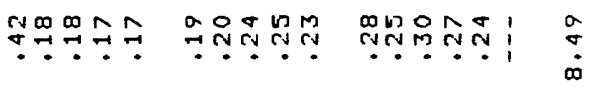

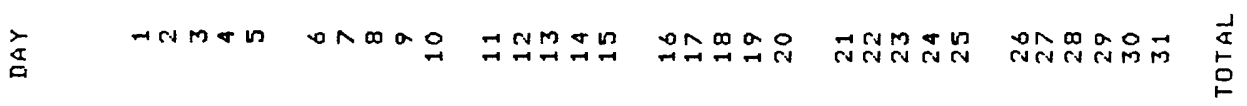




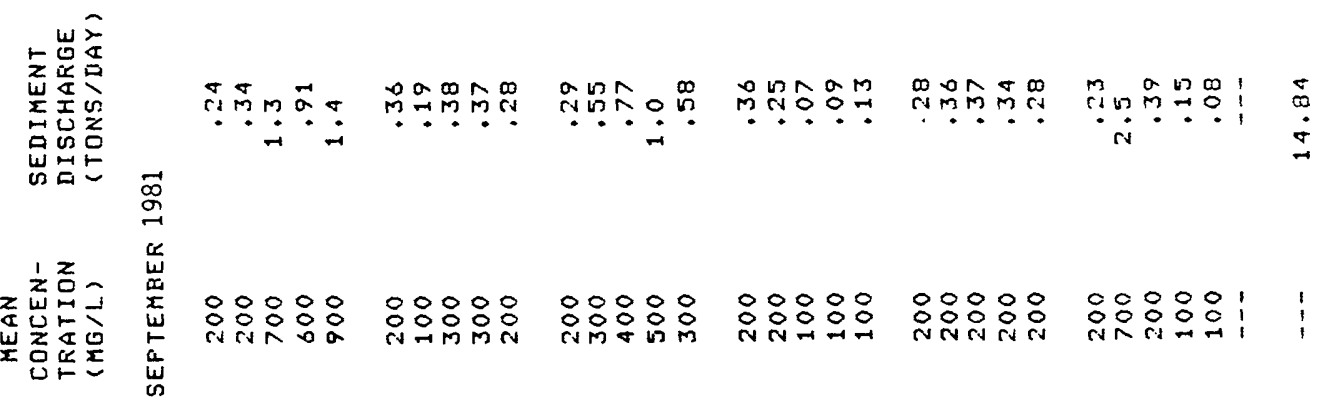

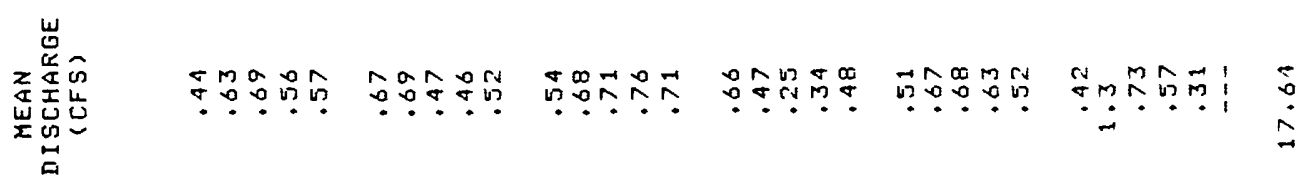

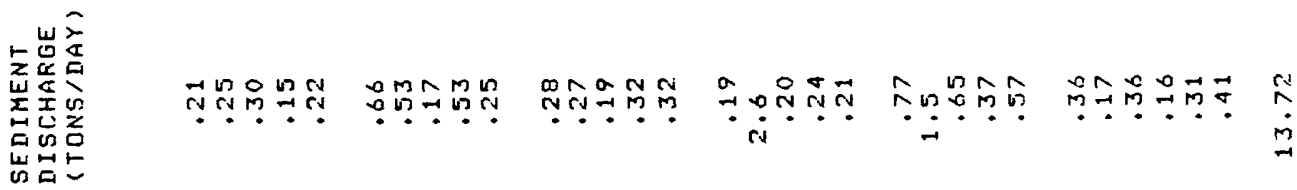

ळ్

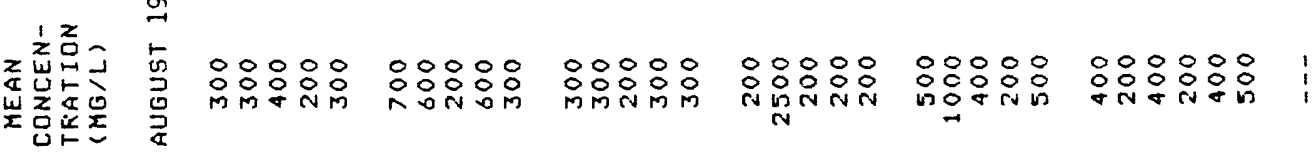

崖

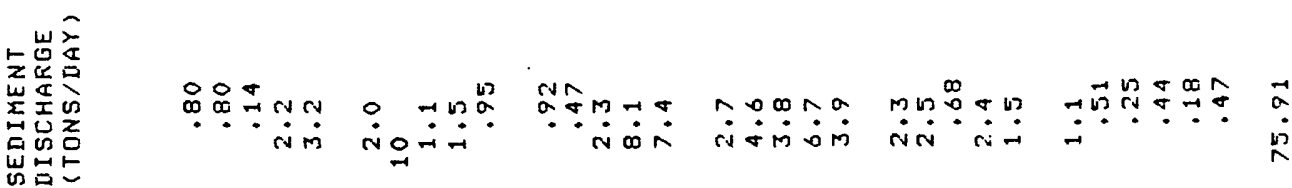

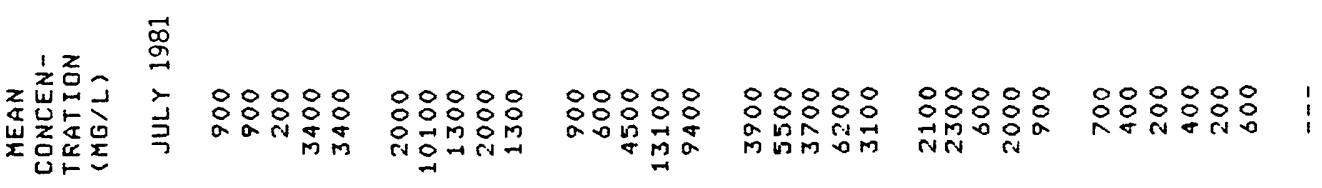

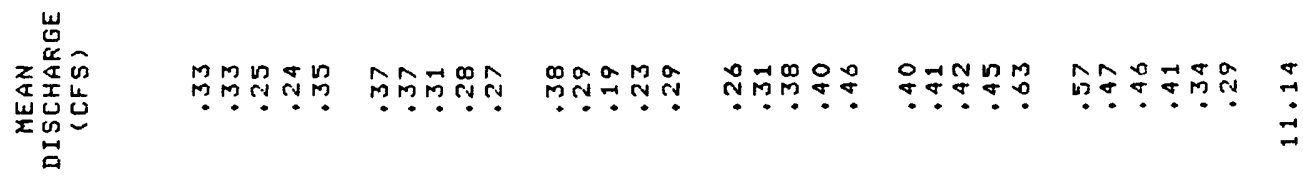

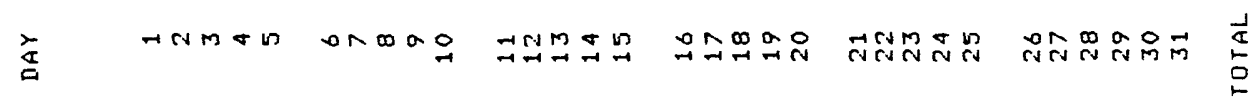




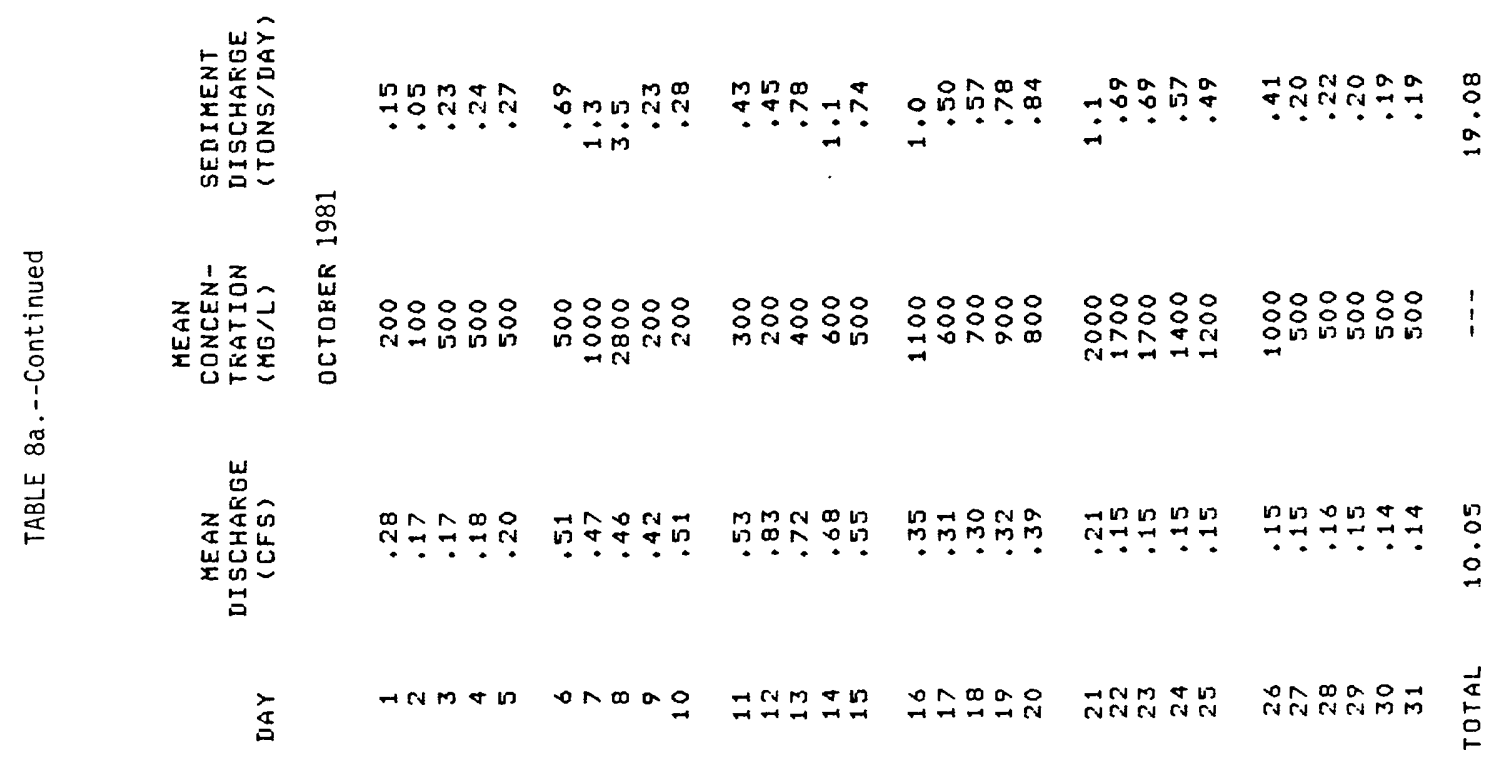




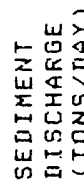

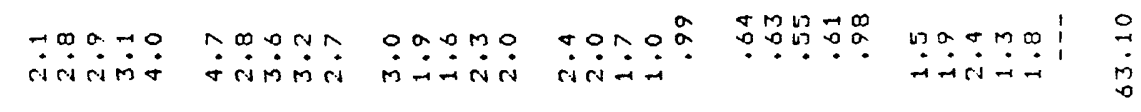

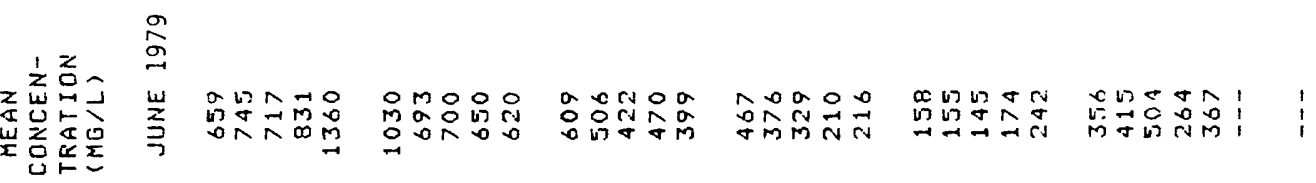

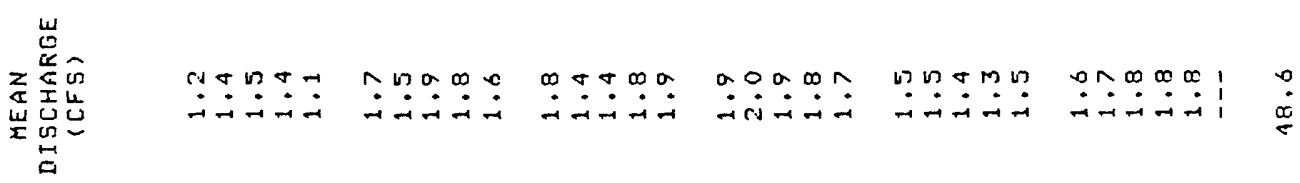

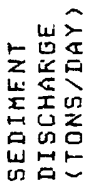

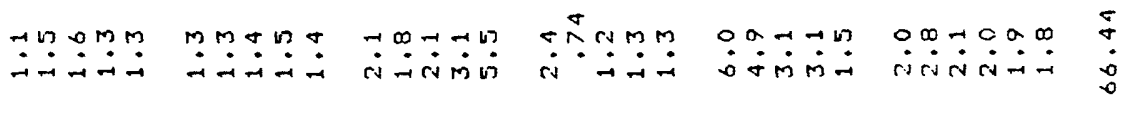

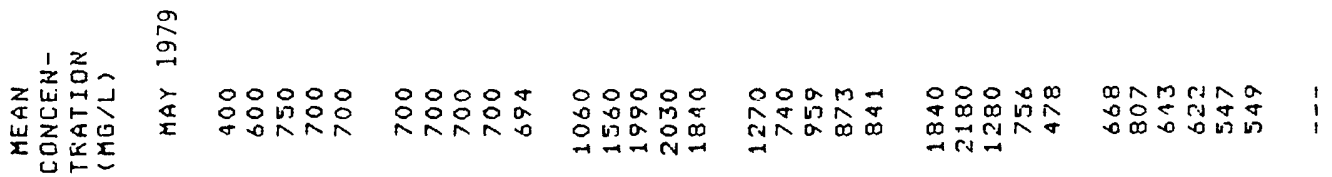

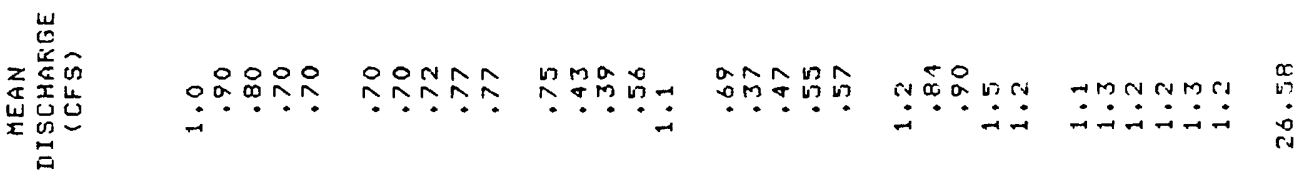

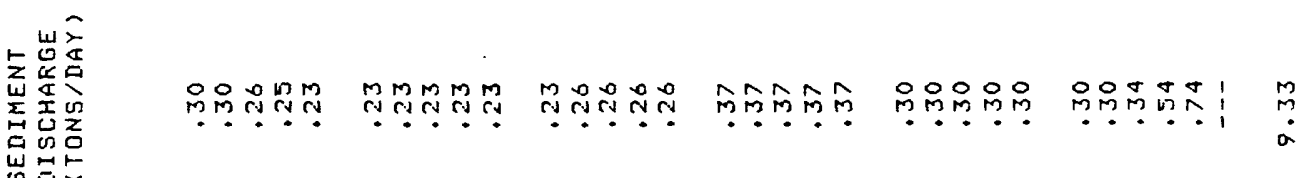

政

$\stackrel{9}{\frac{9}{2}}$

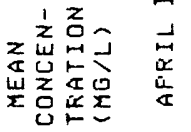

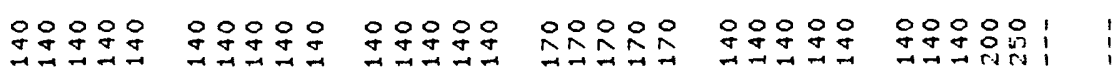

屎

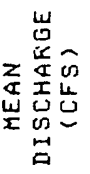

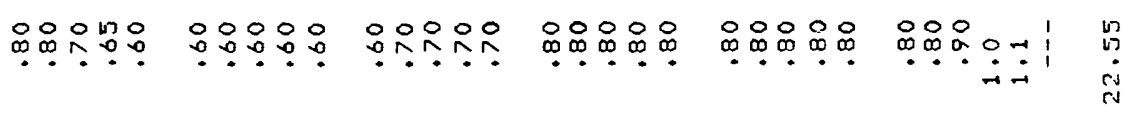

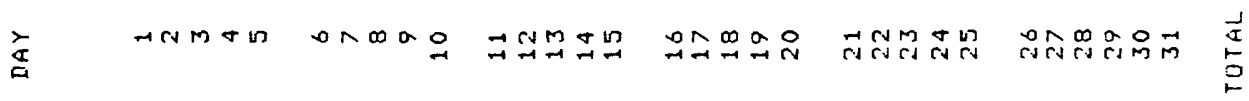




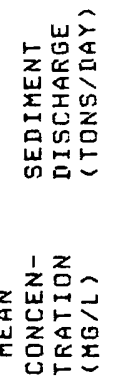

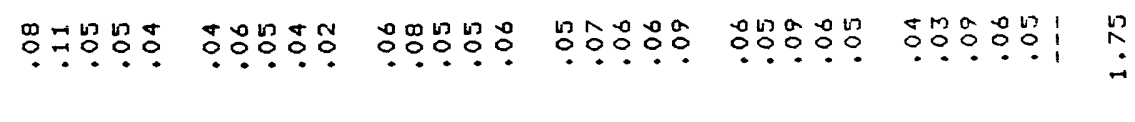

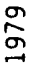

䍃 岕

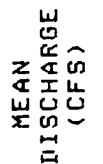

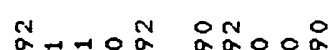

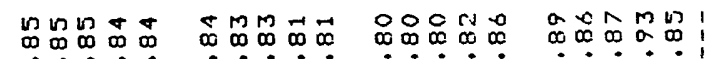

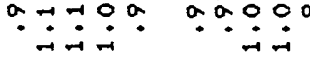

…

$\cdots \cdots$

$\stackrel{2}{i}$

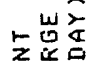

岸茎的

空品品

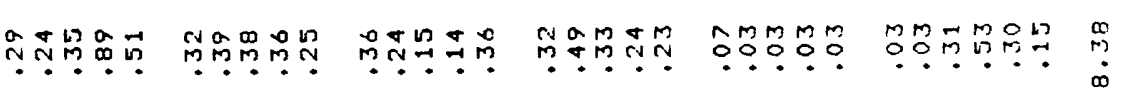

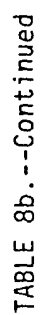

$\stackrel{\circ}{\stackrel{9}{\sigma}}$

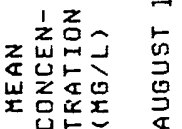

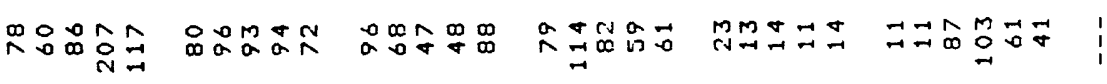

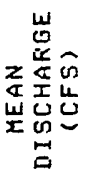

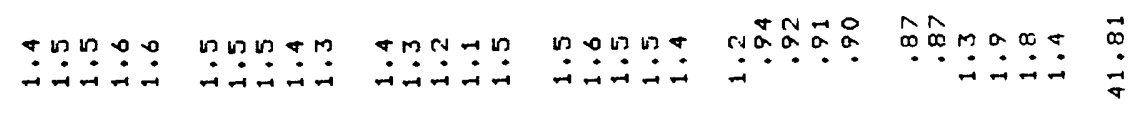

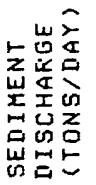

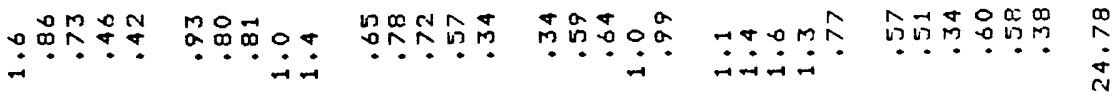

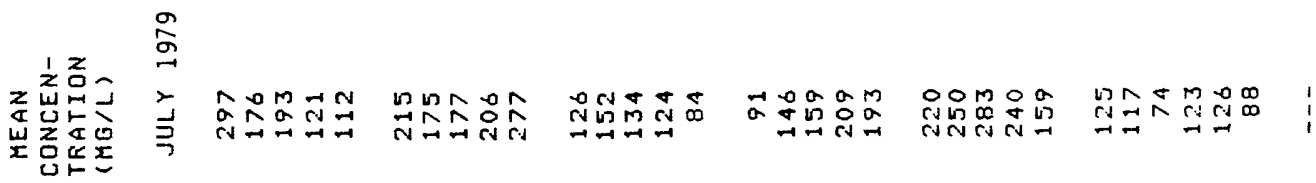

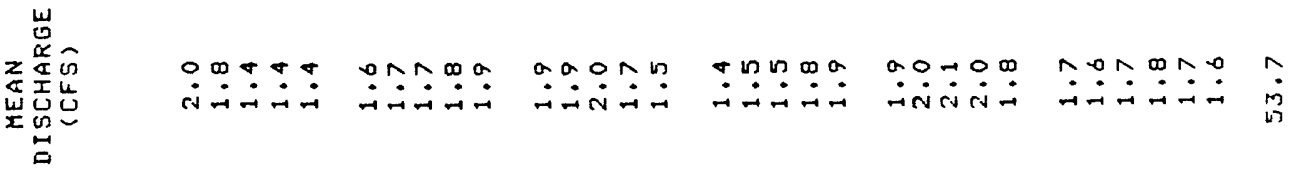

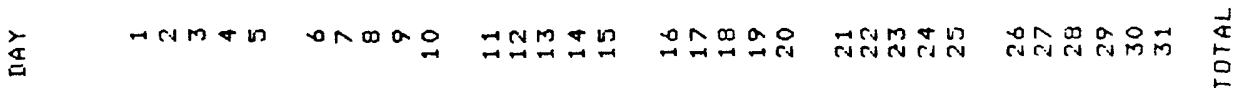




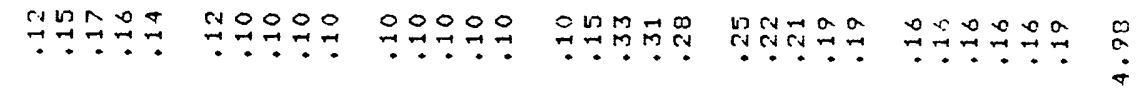

$\stackrel{\circ}{\stackrel{9}{g}}$

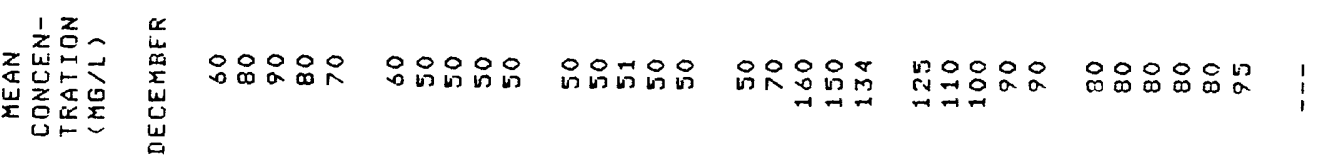

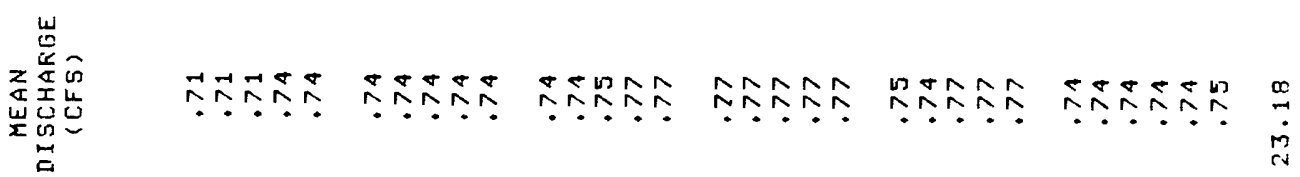

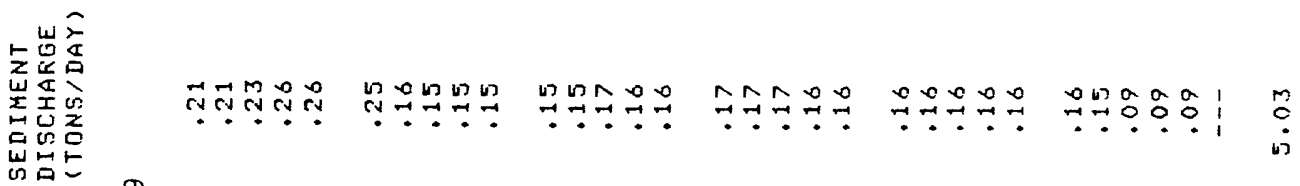

$\stackrel{\text { o. }}{\operatorname{s}}$

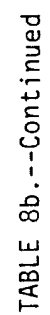

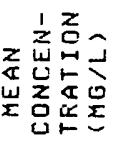

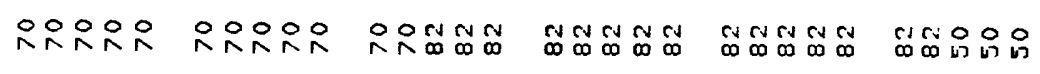

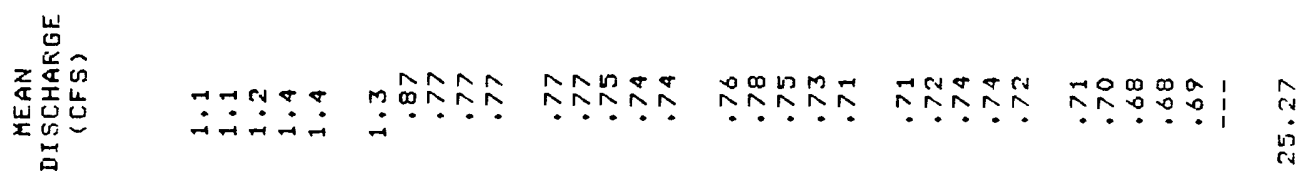

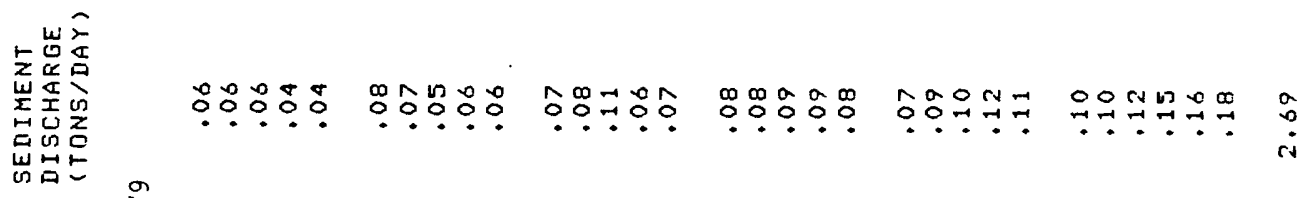

$\stackrel{\circ}{\stackrel{\sigma}{\sigma}}$

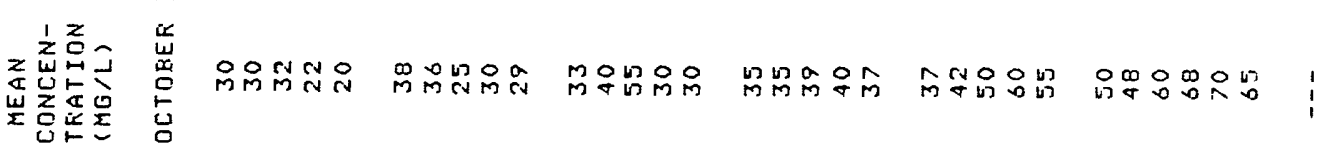

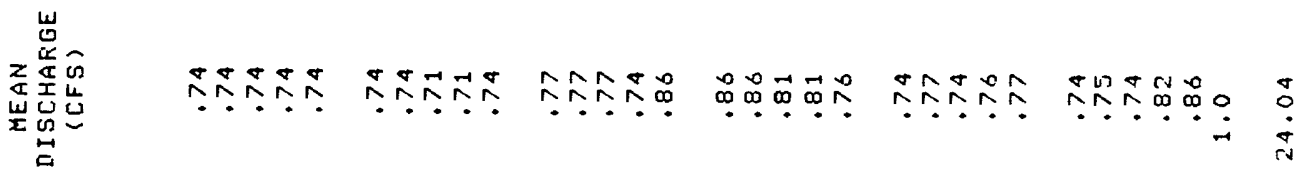

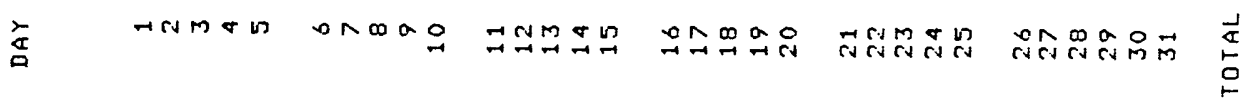




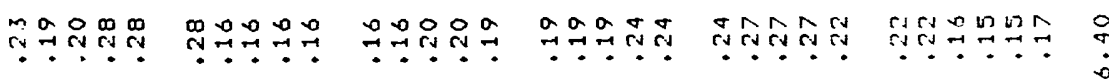

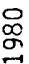

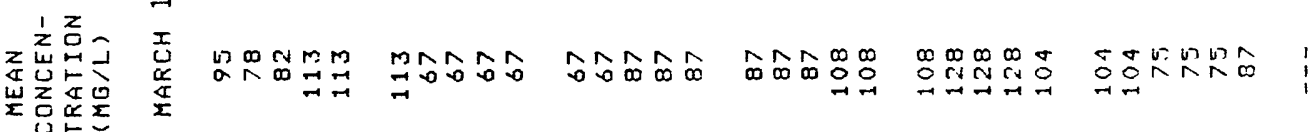

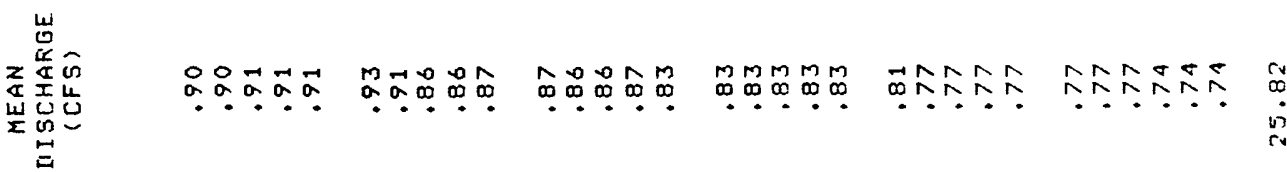

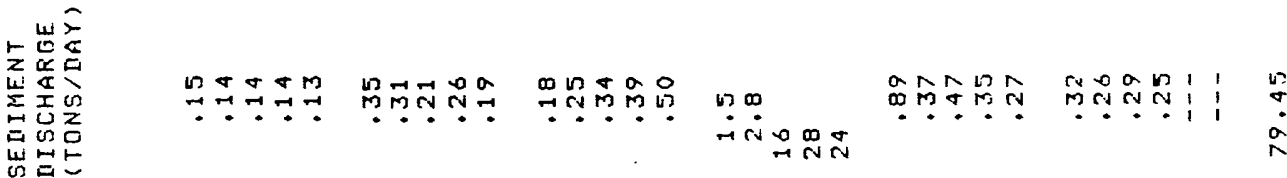

$\stackrel{\circ}{\circ}$

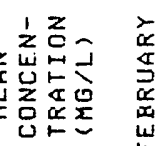

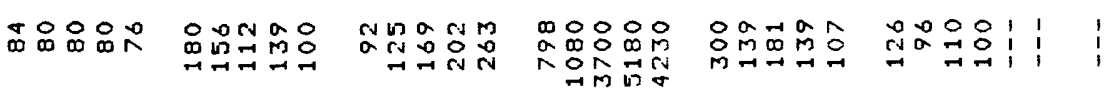

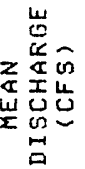

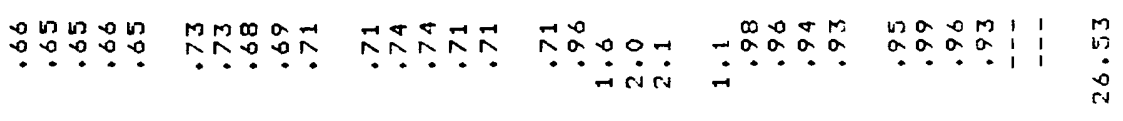

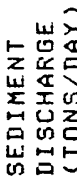

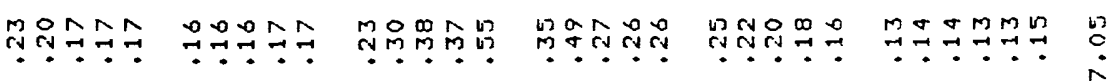

$\stackrel{\circ}{\stackrel{\circ}{\hookrightarrow}}$

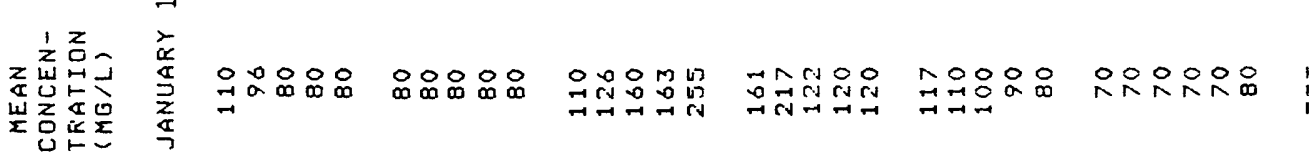

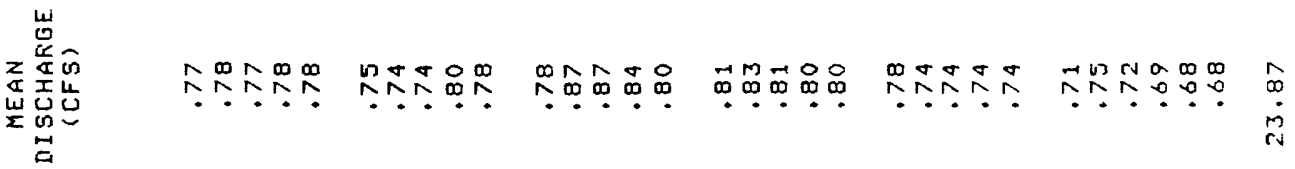

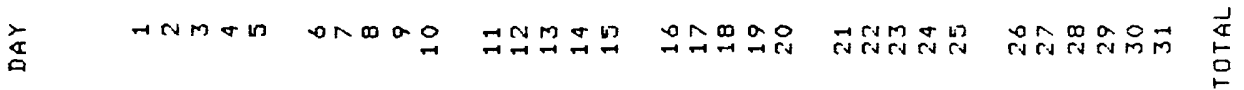




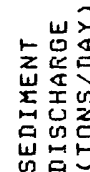

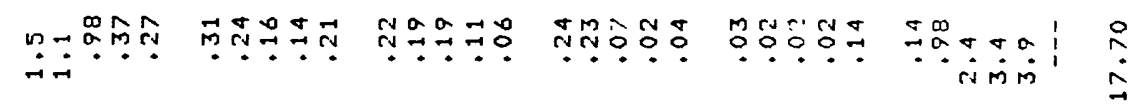

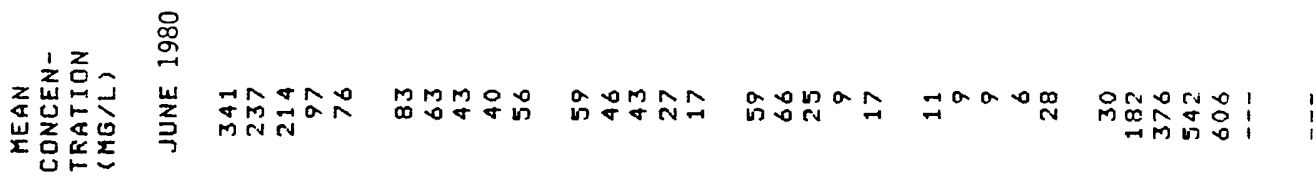

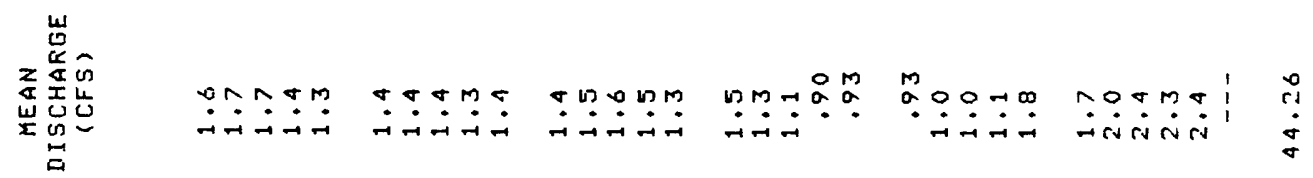

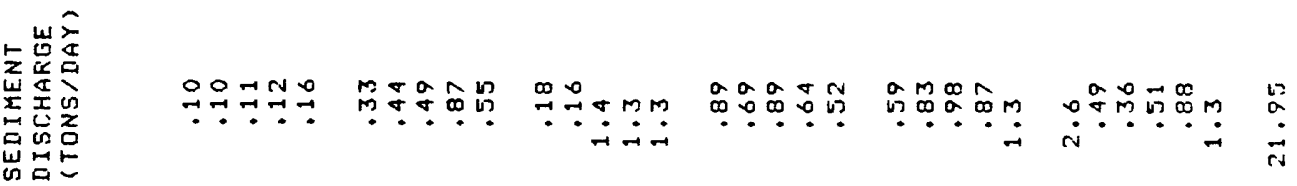

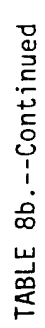

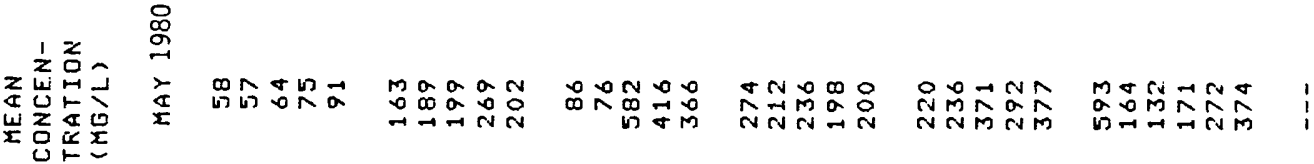

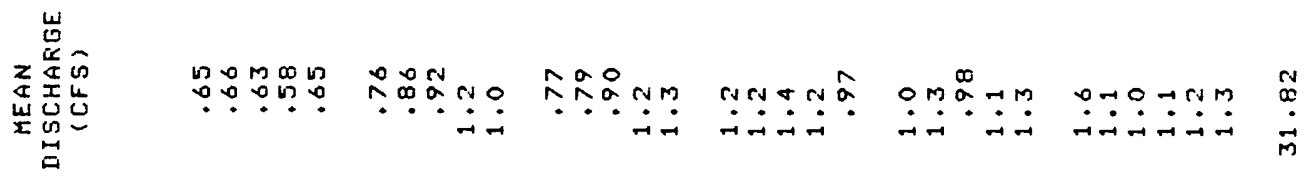

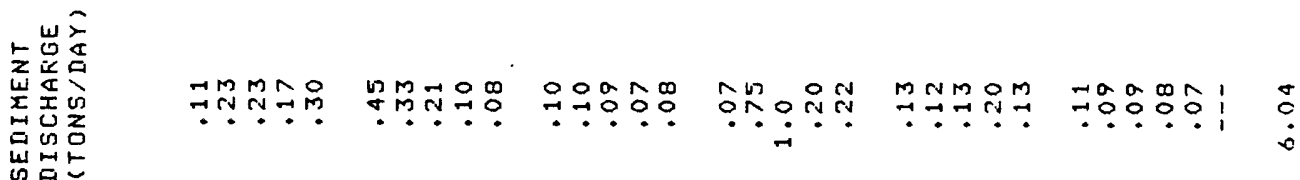

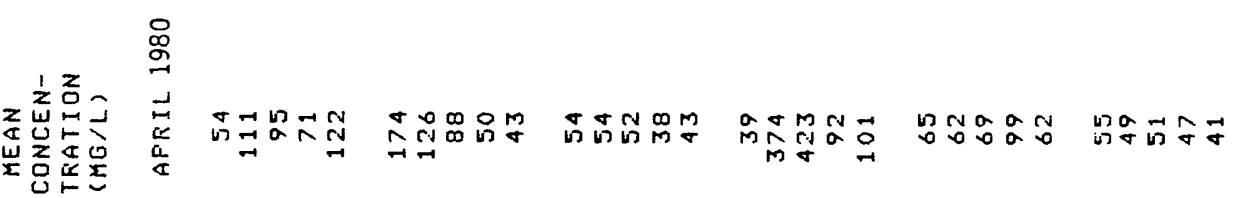

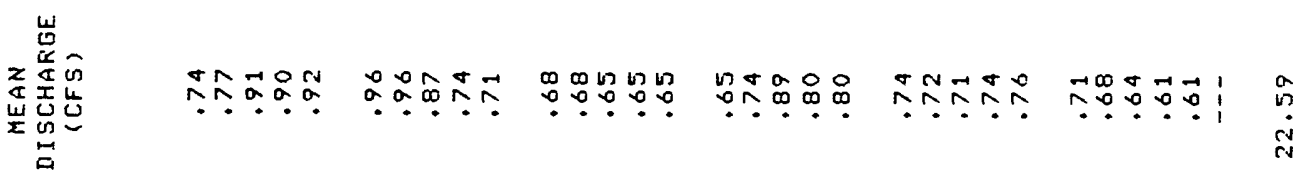

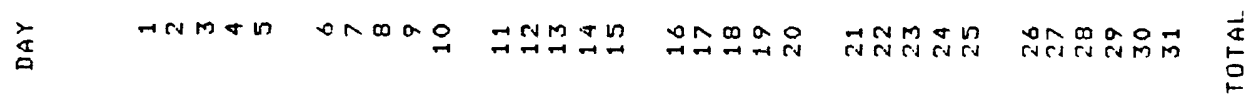




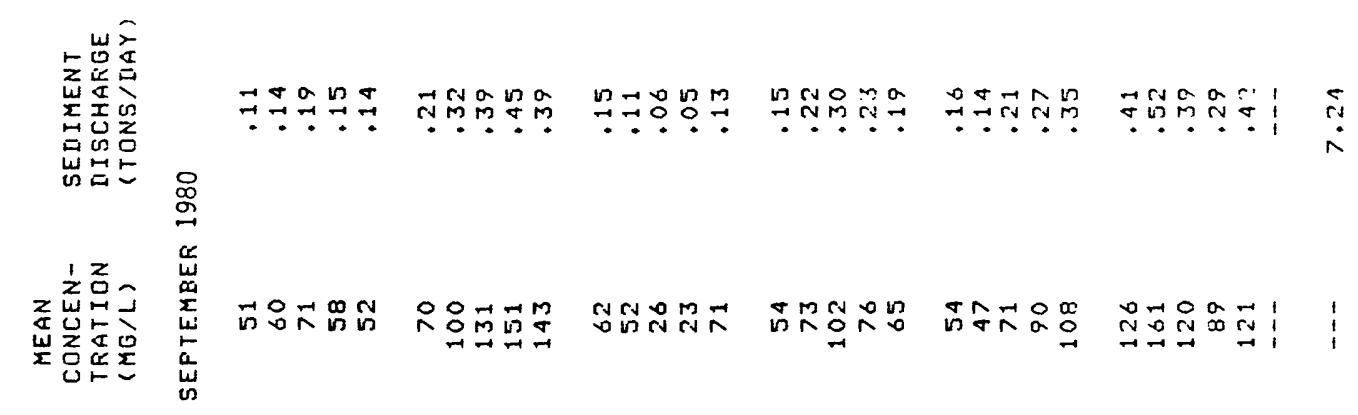

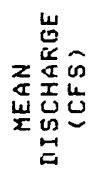

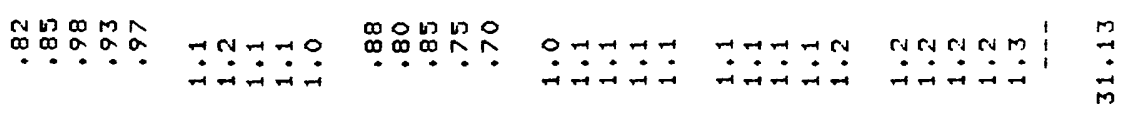

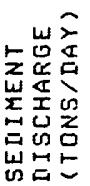

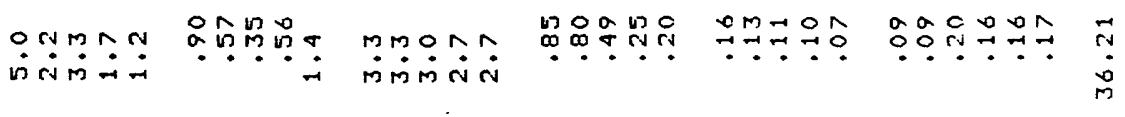

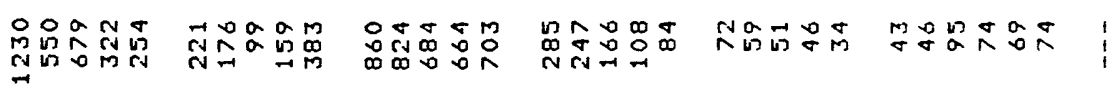
z空过

总

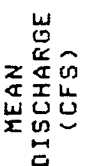

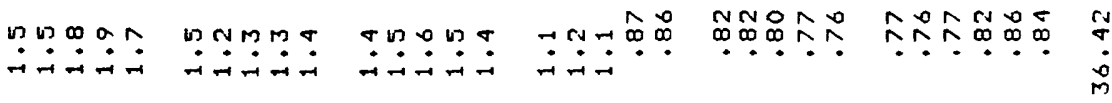
崖崖

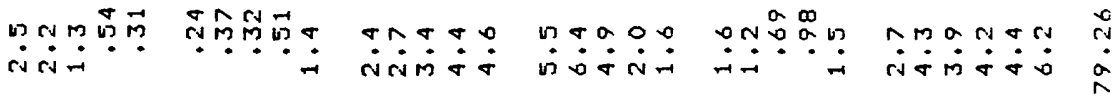

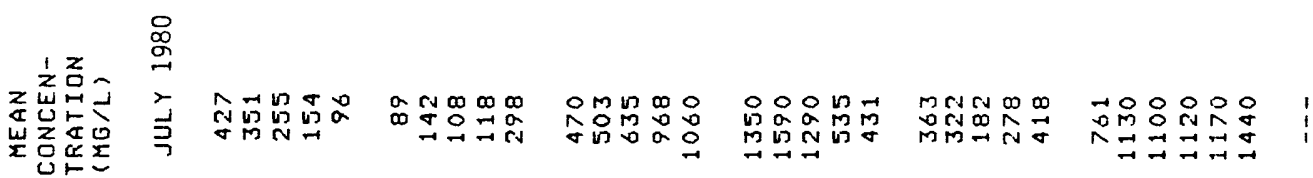

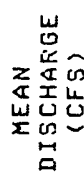

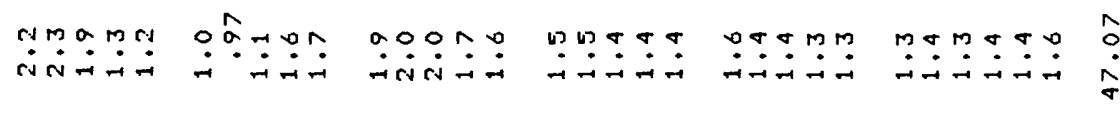

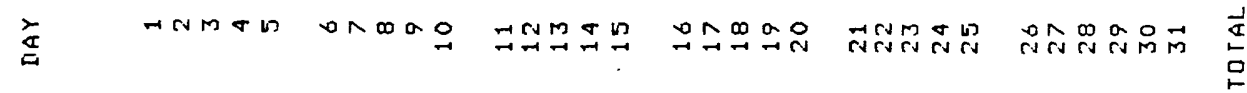




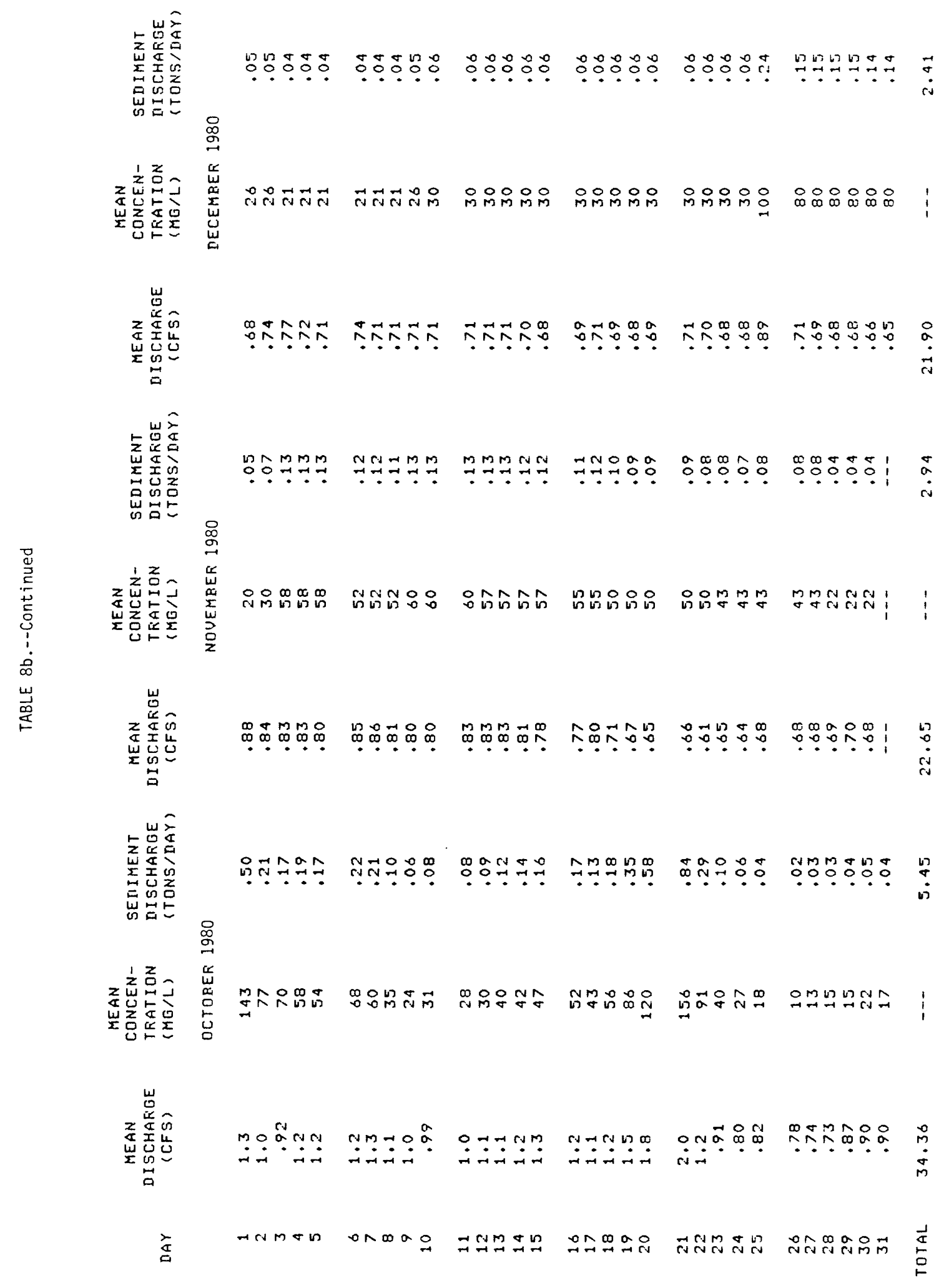




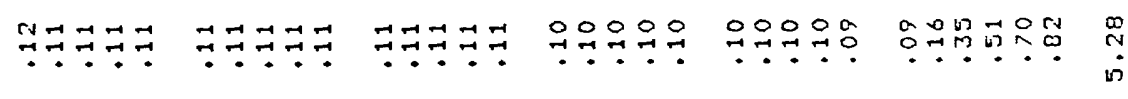

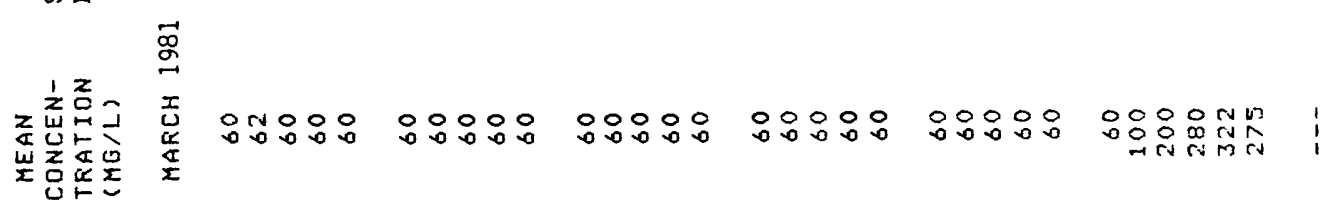

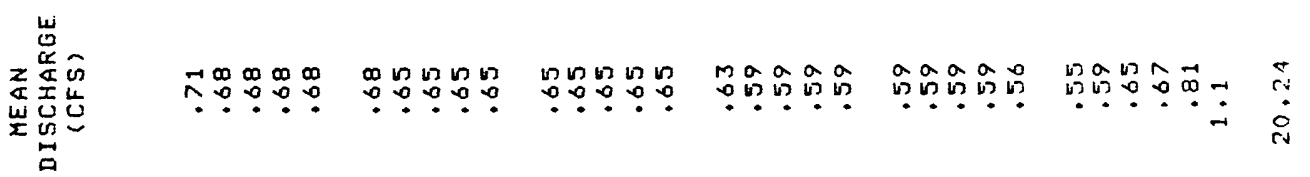

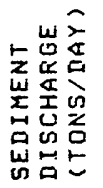

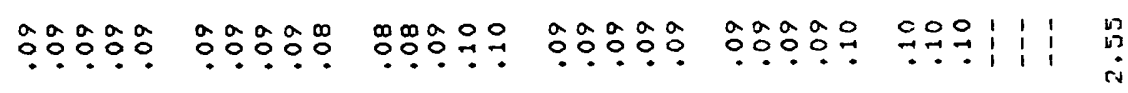
品

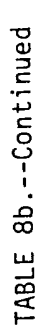

z出恕

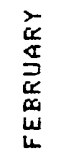

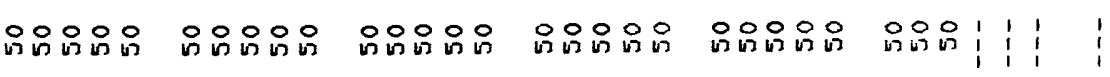

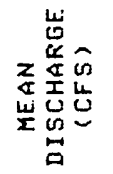

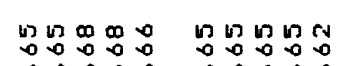

$\sin m=4$

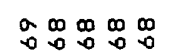

$\infty \infty \infty \infty$

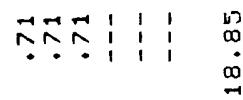

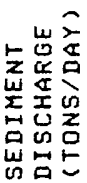

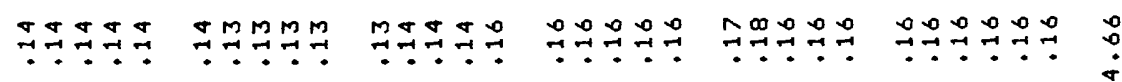

$\stackrel{\vec{\rho}}{\stackrel{\sigma}{\sigma}}$

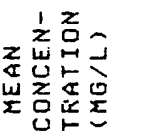

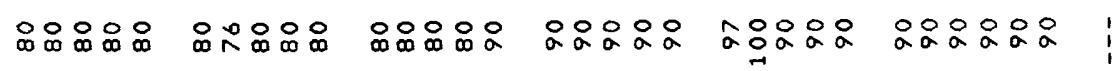

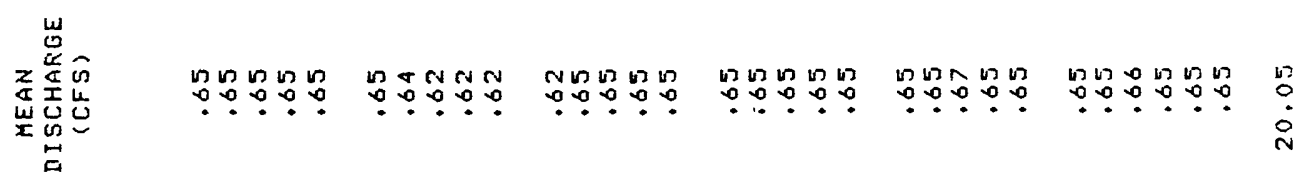

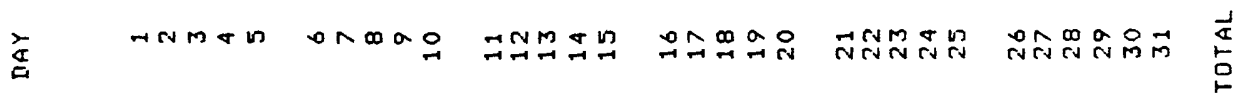




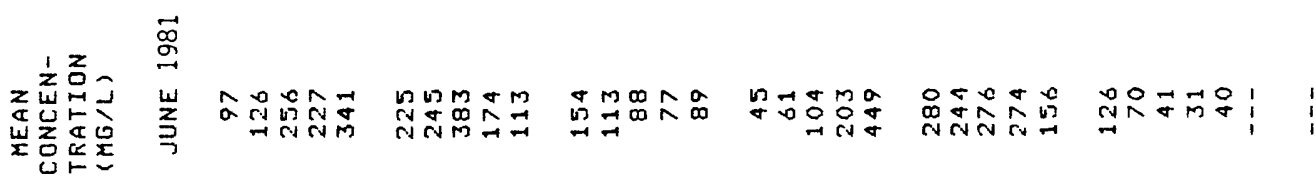

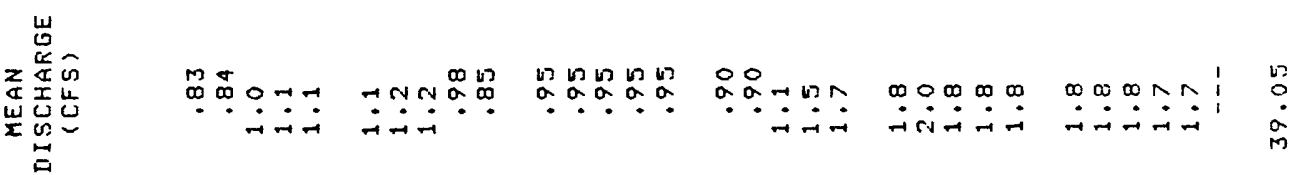

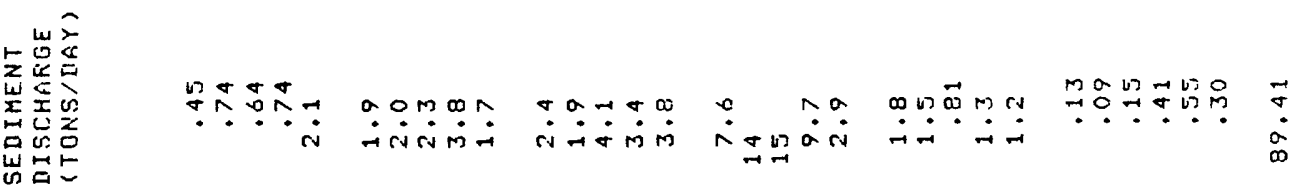

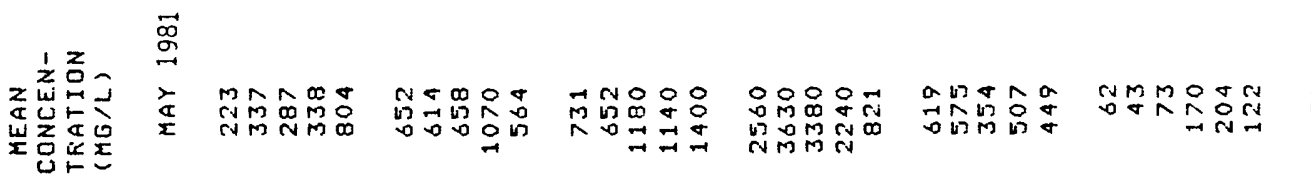

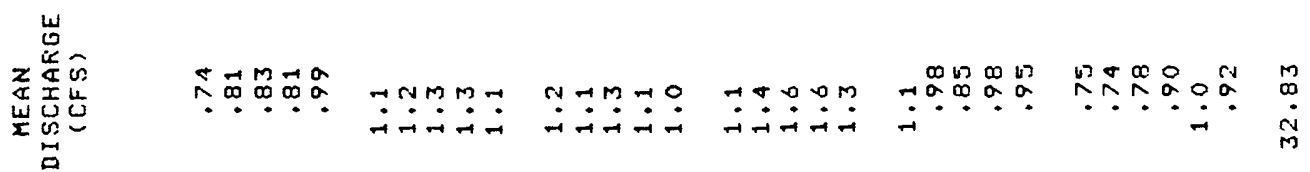

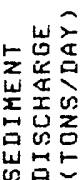

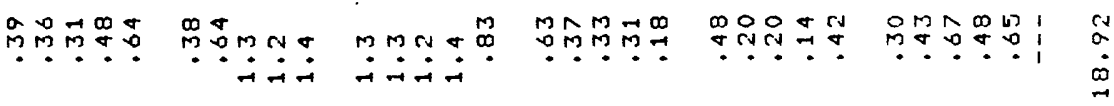

$\stackrel{\overrightarrow{0}}{\rightarrow}$

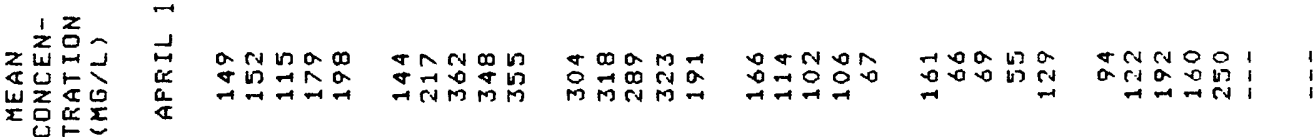

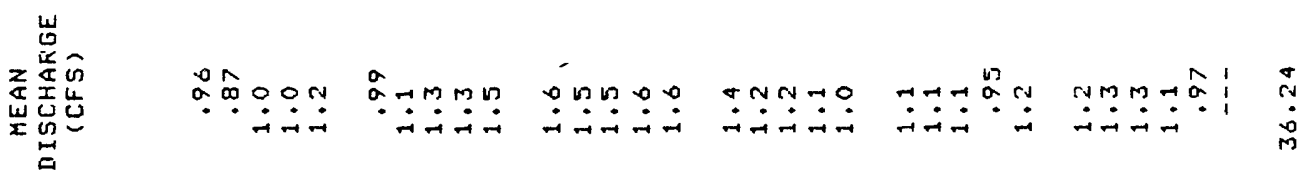

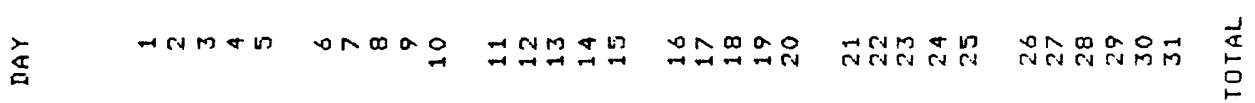




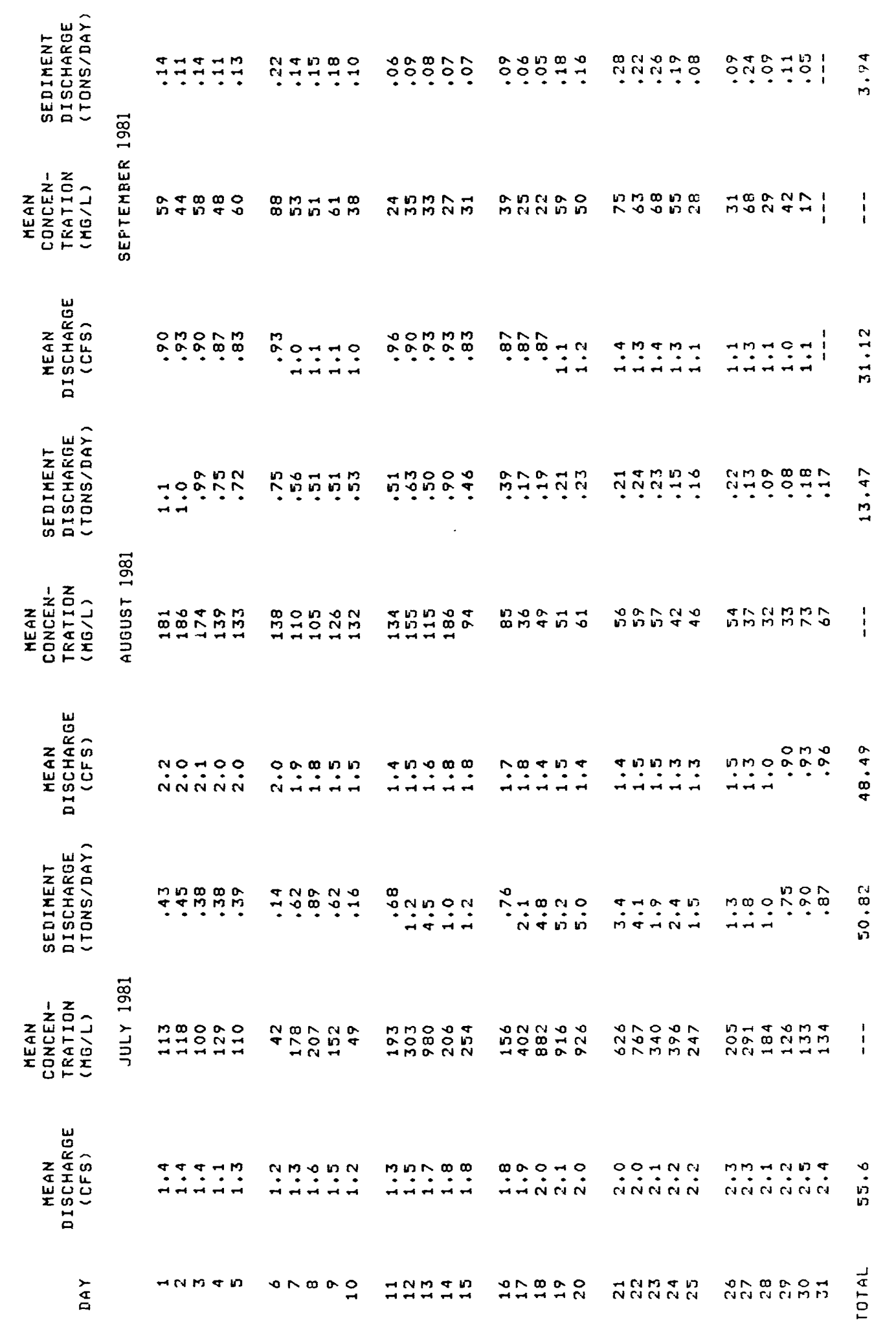




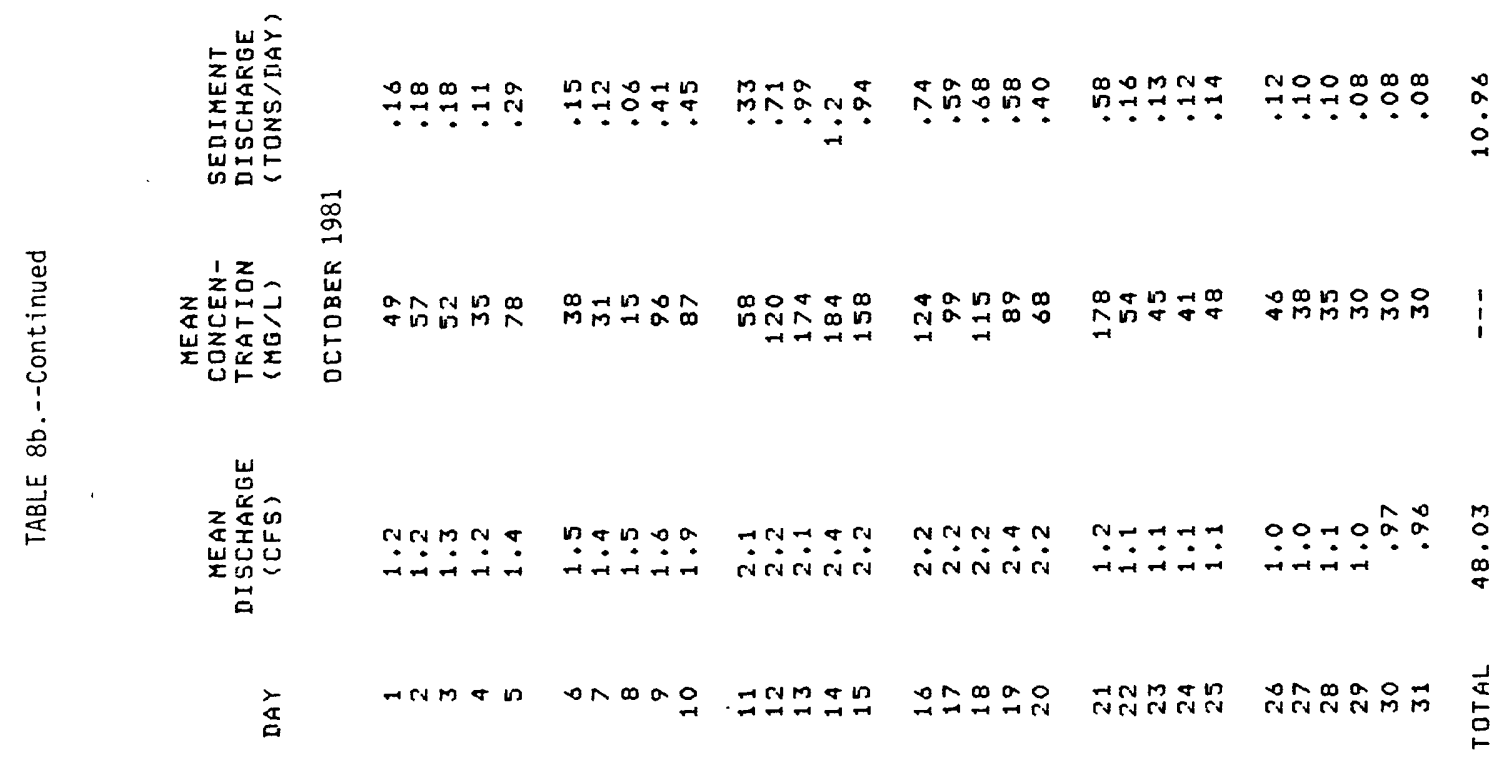


$\stackrel{9}{\stackrel{9}{9}}$

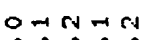

$\tan \cos 0$

Tतy

antoos

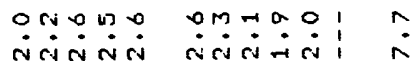

崖怘念

ininisin

arinis

aneñ

anninat

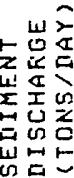

ang $\sin x$

aroog

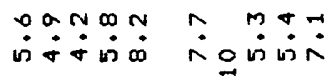

脑的的

लिंकिष

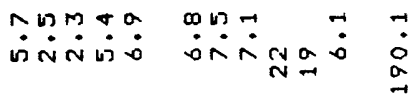

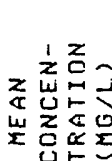

$\stackrel{9}{\stackrel{2}{9}}$

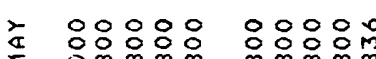

我品管

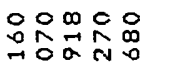

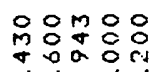

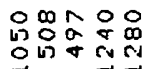

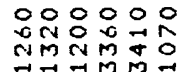

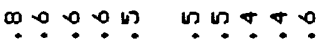

mกñ⿻ otror

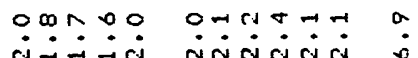

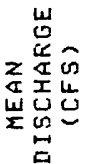

निन्म

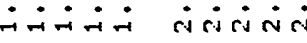

तiनiनi

annisira

岩文

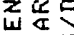

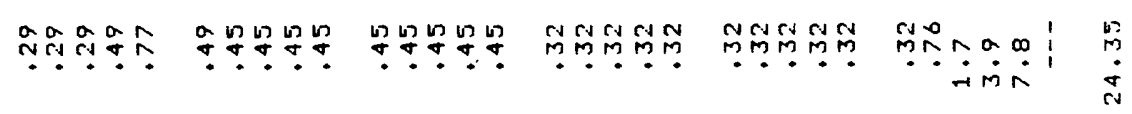

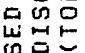

$\frac{1}{2}$

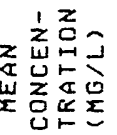

웅응요문

웅영영영

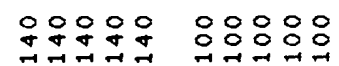

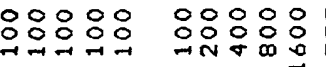

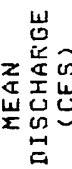

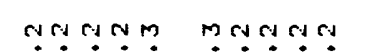

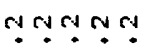

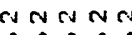

yed

$\stackrel{\circ}{\infty}$

$\stackrel{2}{a}$

$\operatorname{tanan}$

anoo의 $\Rightarrow$ am

an

$\cos 2 \cos 20$

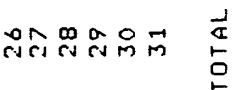




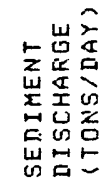

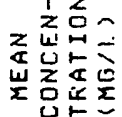

a

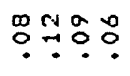

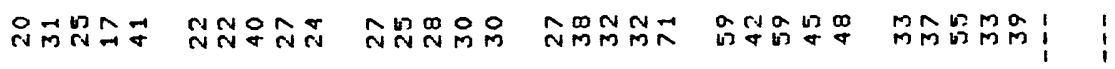

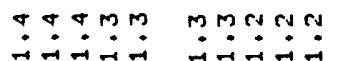

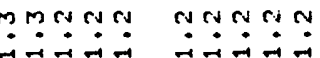

$\sin : \sin$

$\because \because \because \div$

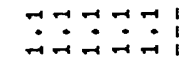

$\dot{0}$

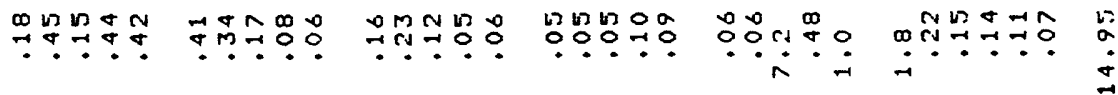

के

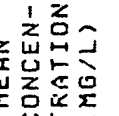

$\stackrel{5}{\frac{5}{2}}$

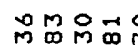

ดิธกตำ

किष्ष

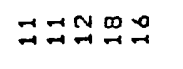

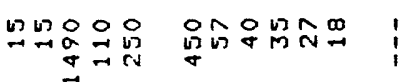

aogors

irifiri

Metion

$\because a ? ? ?$

$\because \cong 000$

$\because \because 00$

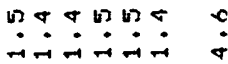

幽它

岁宅

必造

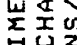

包恕各

ํำํำษ

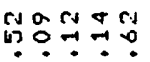

ษ

วับุ์?

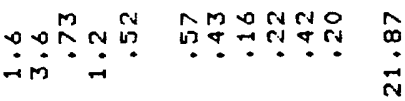

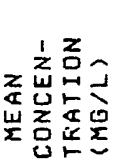

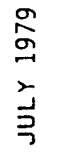

a d

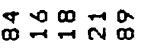

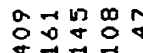

$\vec{m} \stackrel{\sigma}{0} \underset{\alpha}{N}$

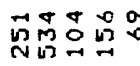

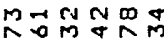

崖

mrsmma arisin

$\operatorname{mos} 200$

manoo

$0090 m$

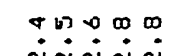

a oa a o

nanisi ricicina

numin

Niñí

nNंi

㹸

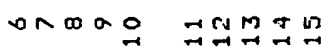

$\stackrel{0 \rightarrow a}{a}$

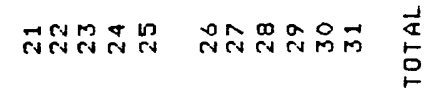




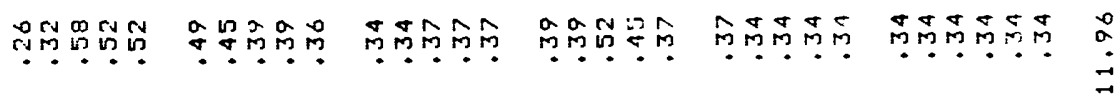

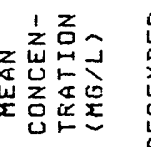

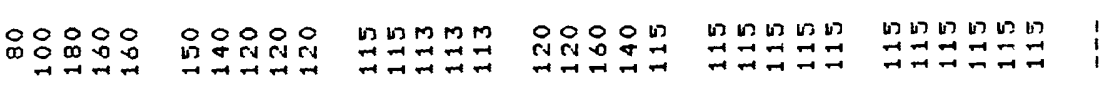

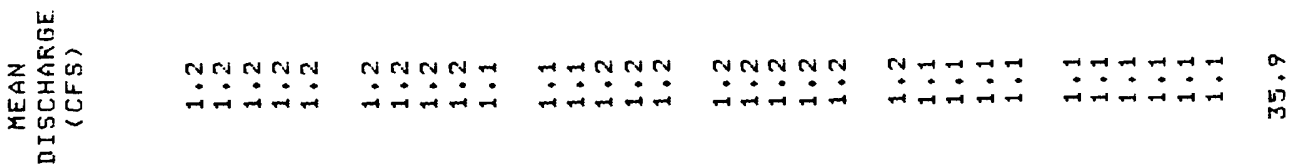

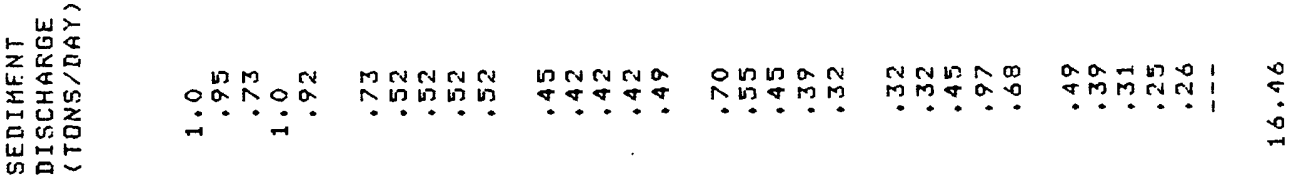

$\stackrel{9}{9}$

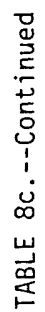

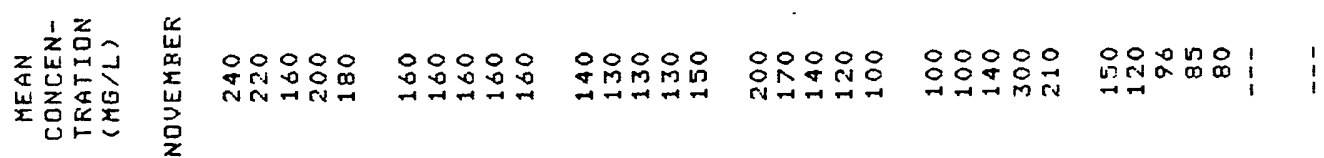

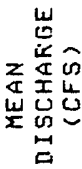

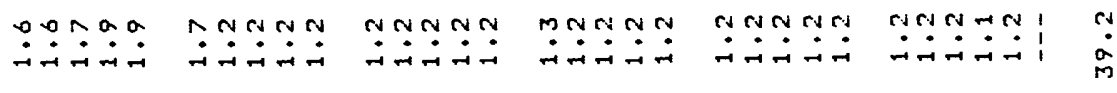

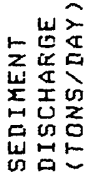

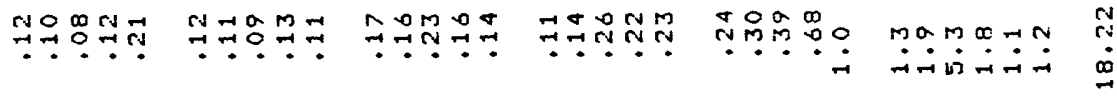

$\stackrel{9}{\stackrel{9}{9}}$

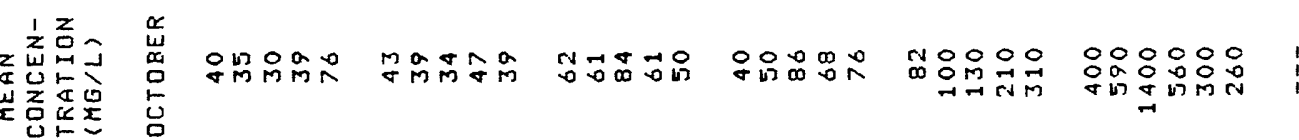

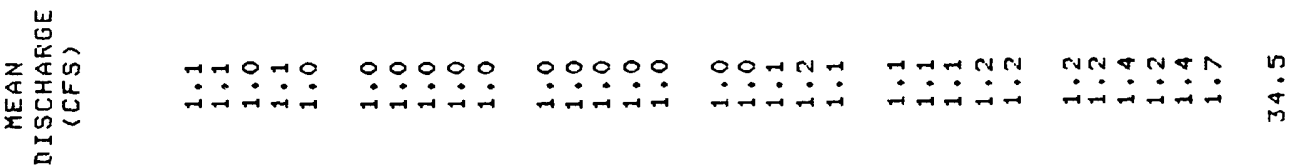

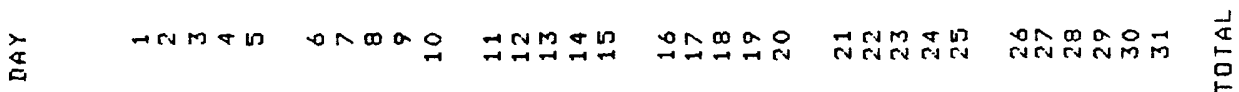




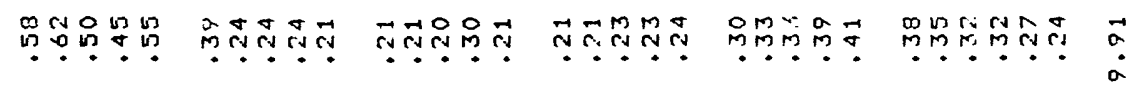

$\underset{\substack{0 \\ \hdashline}}{9}$

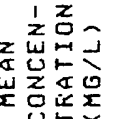

$\frac{I}{\frac{\pi}{L}}$

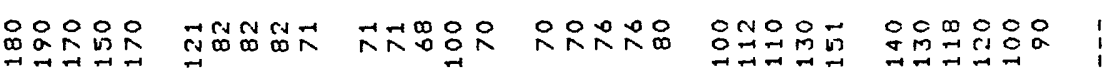

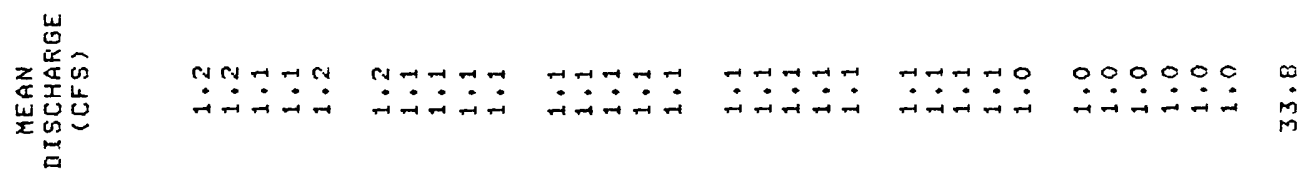

点岕亲

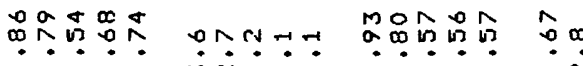

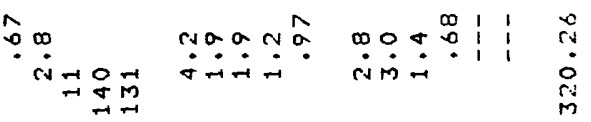

$\stackrel{\circ}{\stackrel{\circ}{9}}$

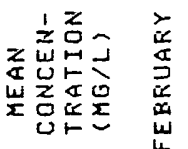

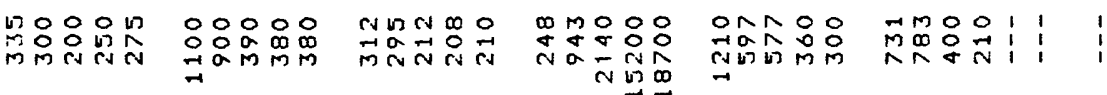

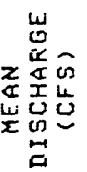

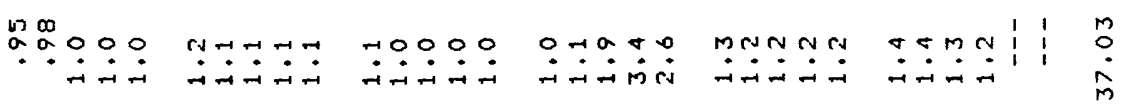

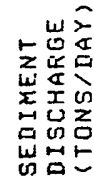

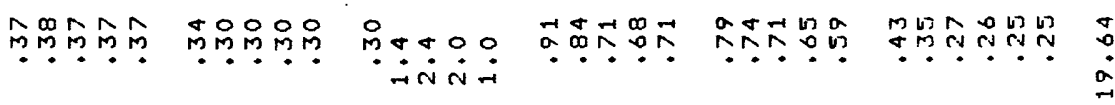

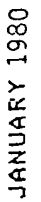

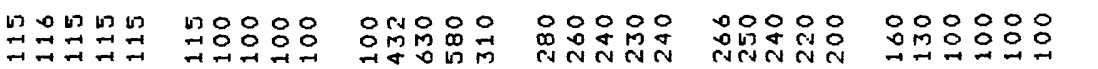

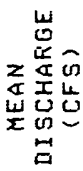

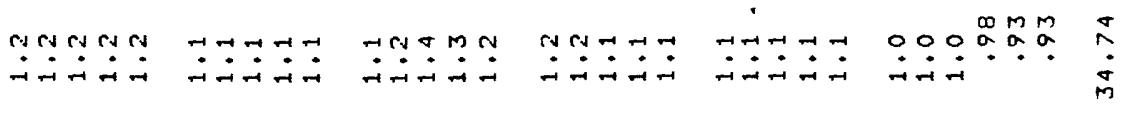

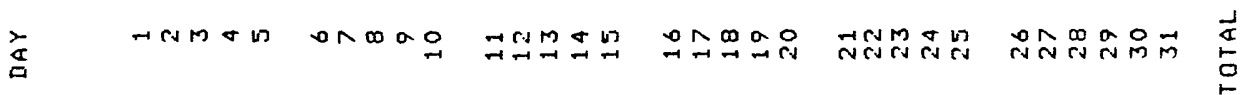




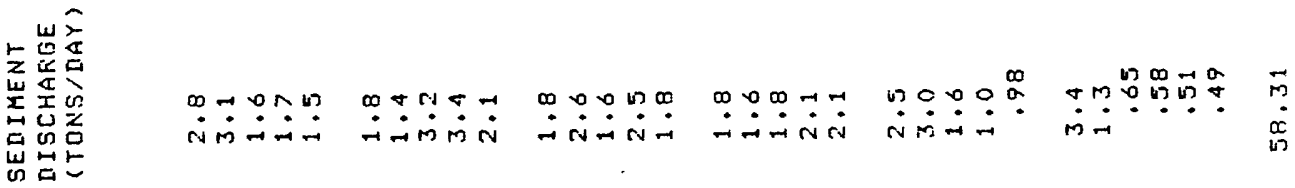

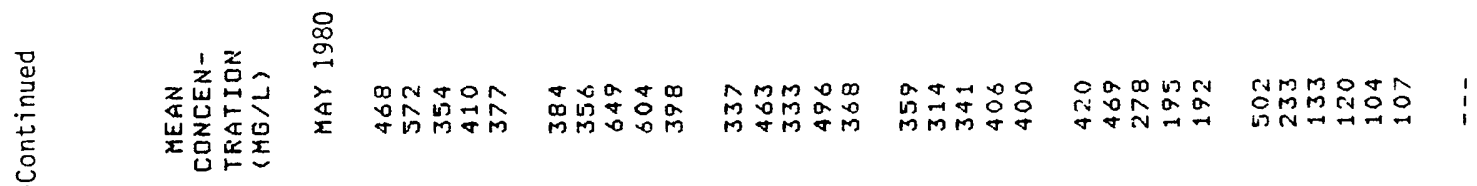

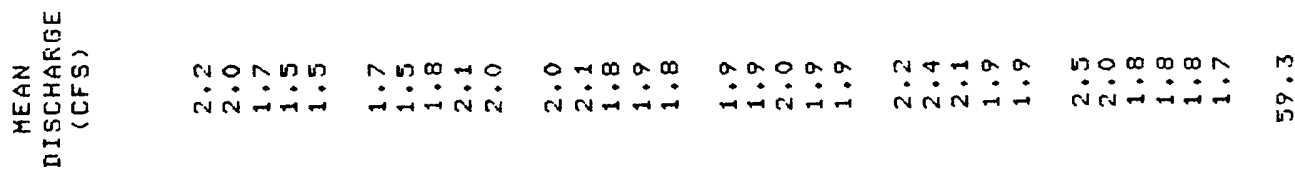

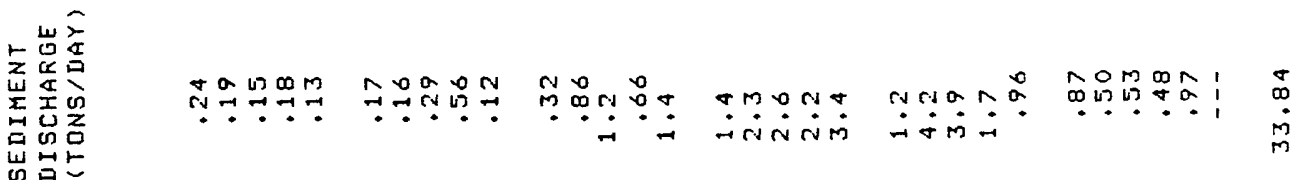

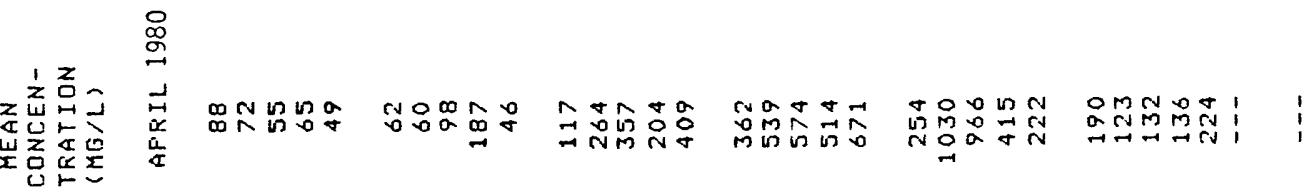

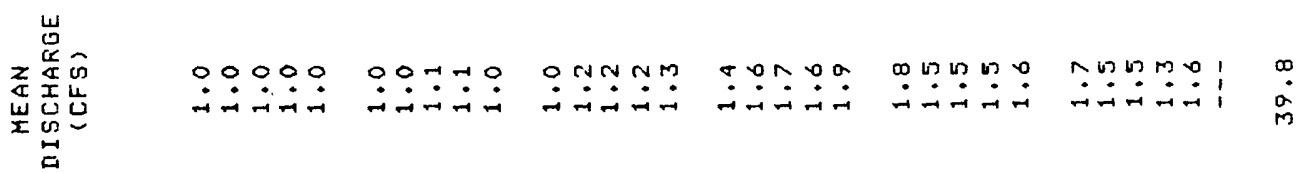

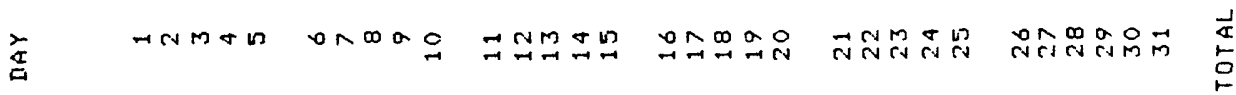




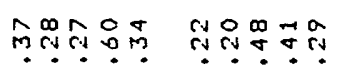

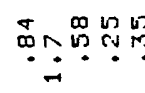

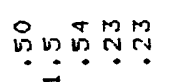

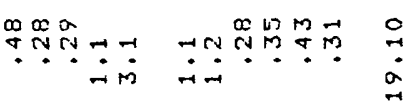

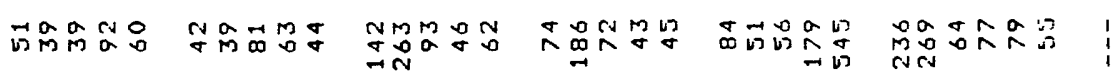

㟧

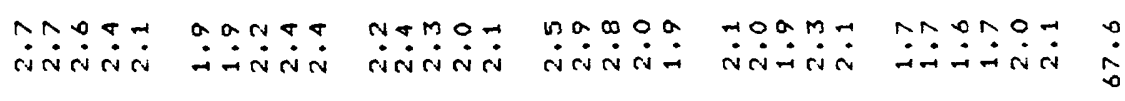

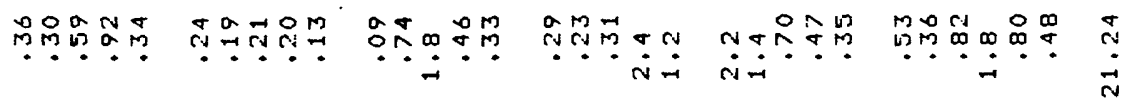

$\stackrel{\circ}{\circ}$ 空䂞

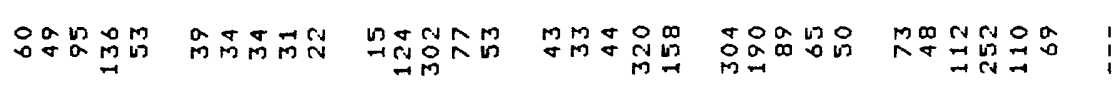

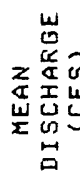
chmmera mamora cannam $\ln 0000$

nnano nonnaod

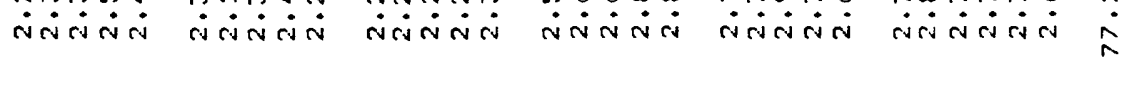

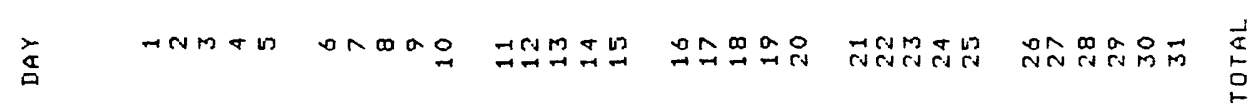




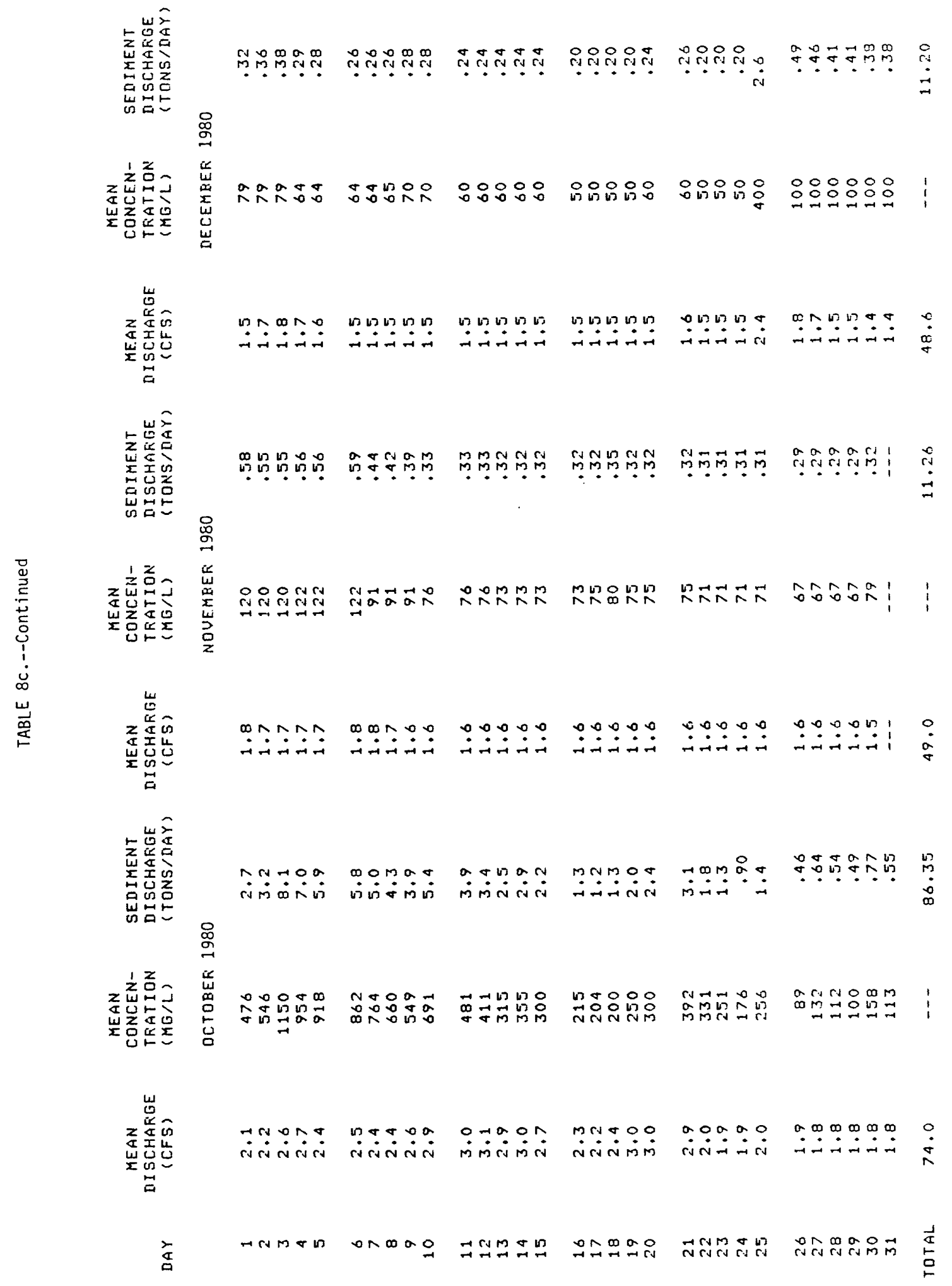




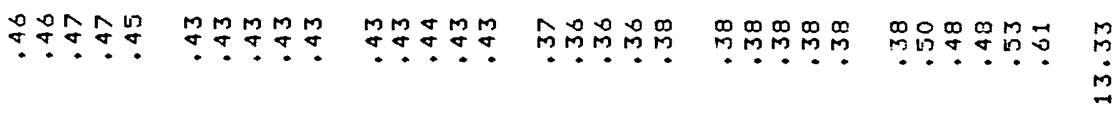

$\vec{\infty}$

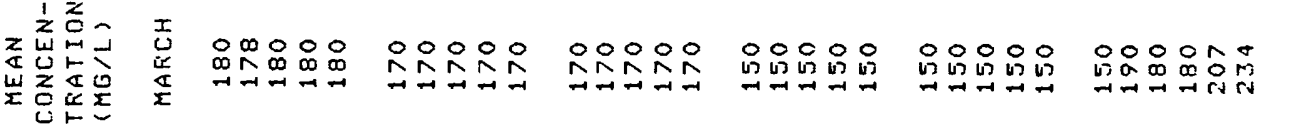

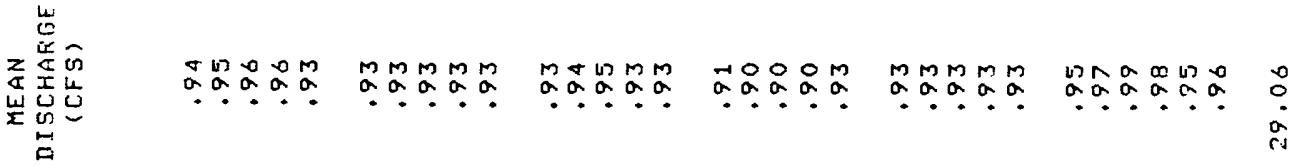

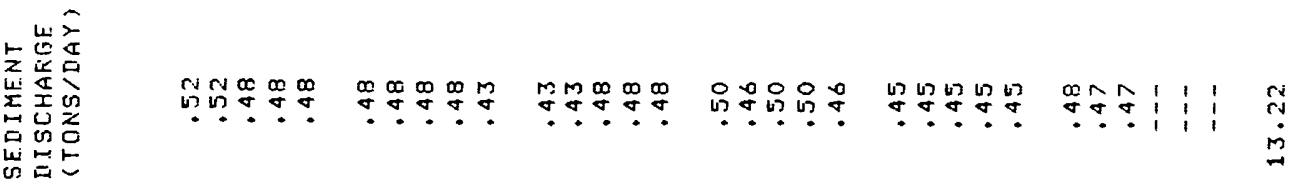

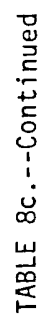

$\stackrel{\vec{\infty}}{\stackrel{-}{\rightarrow}}$

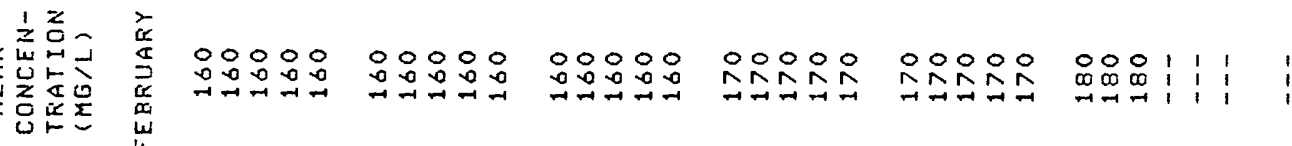

㟶

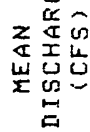

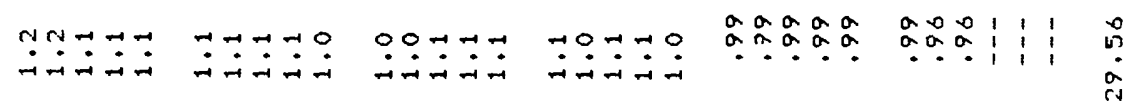

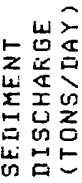

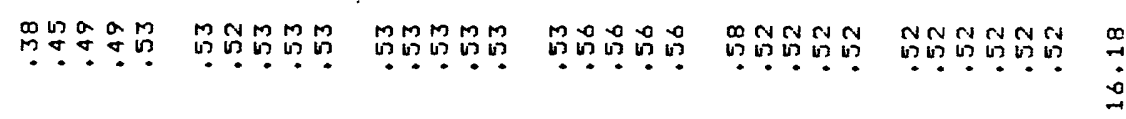

$\overrightarrow{\mathrm{o}}$

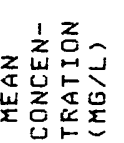

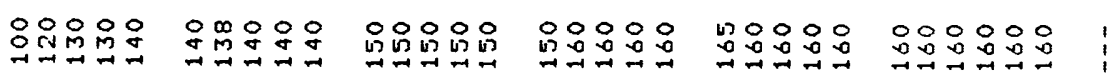

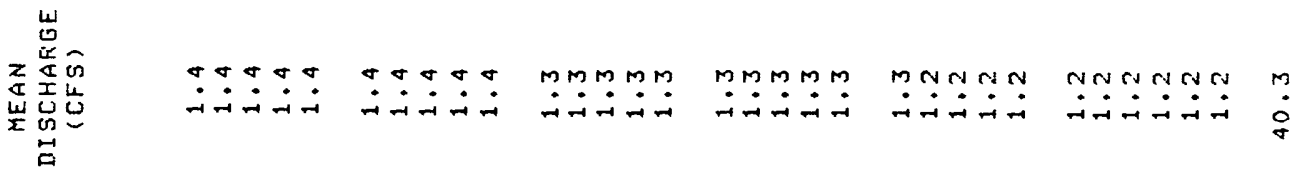

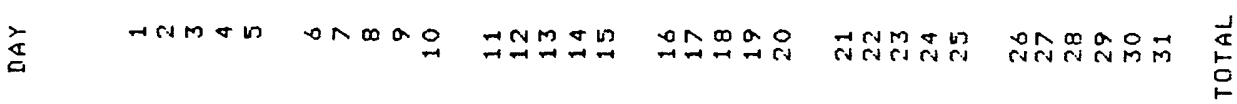




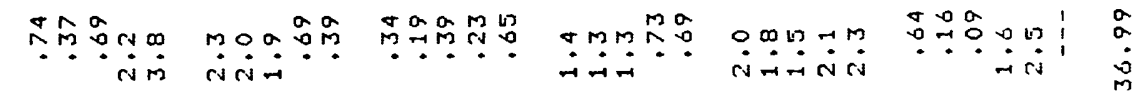

$\vec{D}$

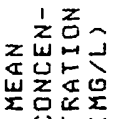

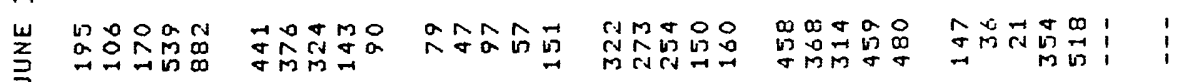

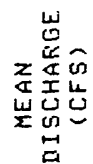

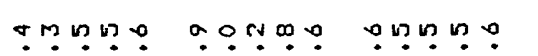

an $\infty$

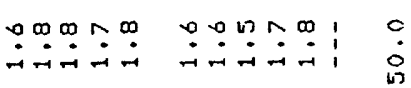

เ宅宅

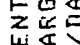

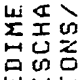

品必我

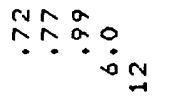

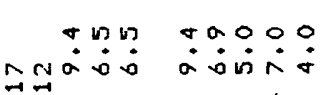

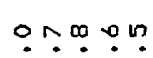

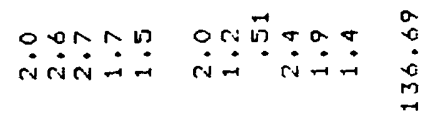

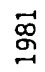

文范

I

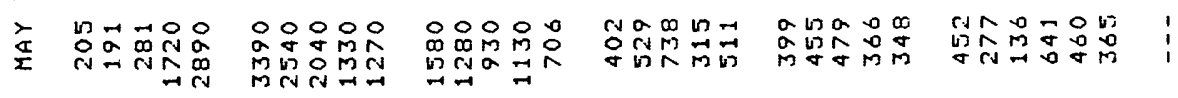

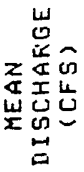

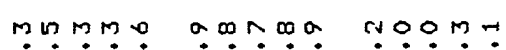

कaaal anபே்-

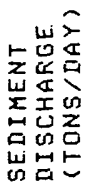

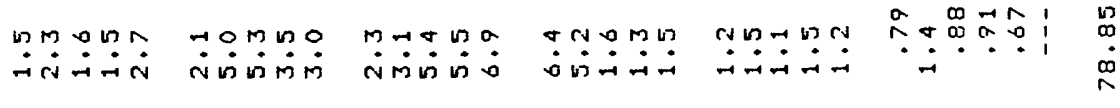

$\vec{\infty}$

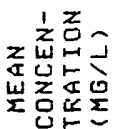

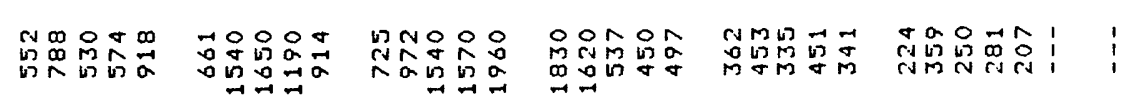

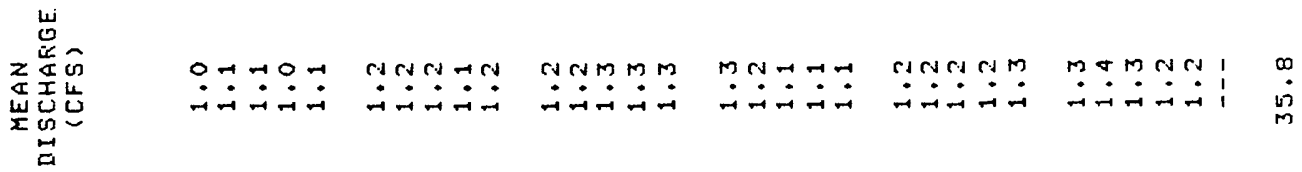

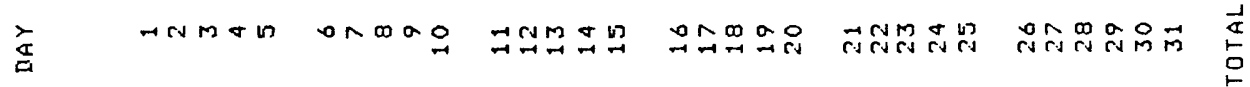




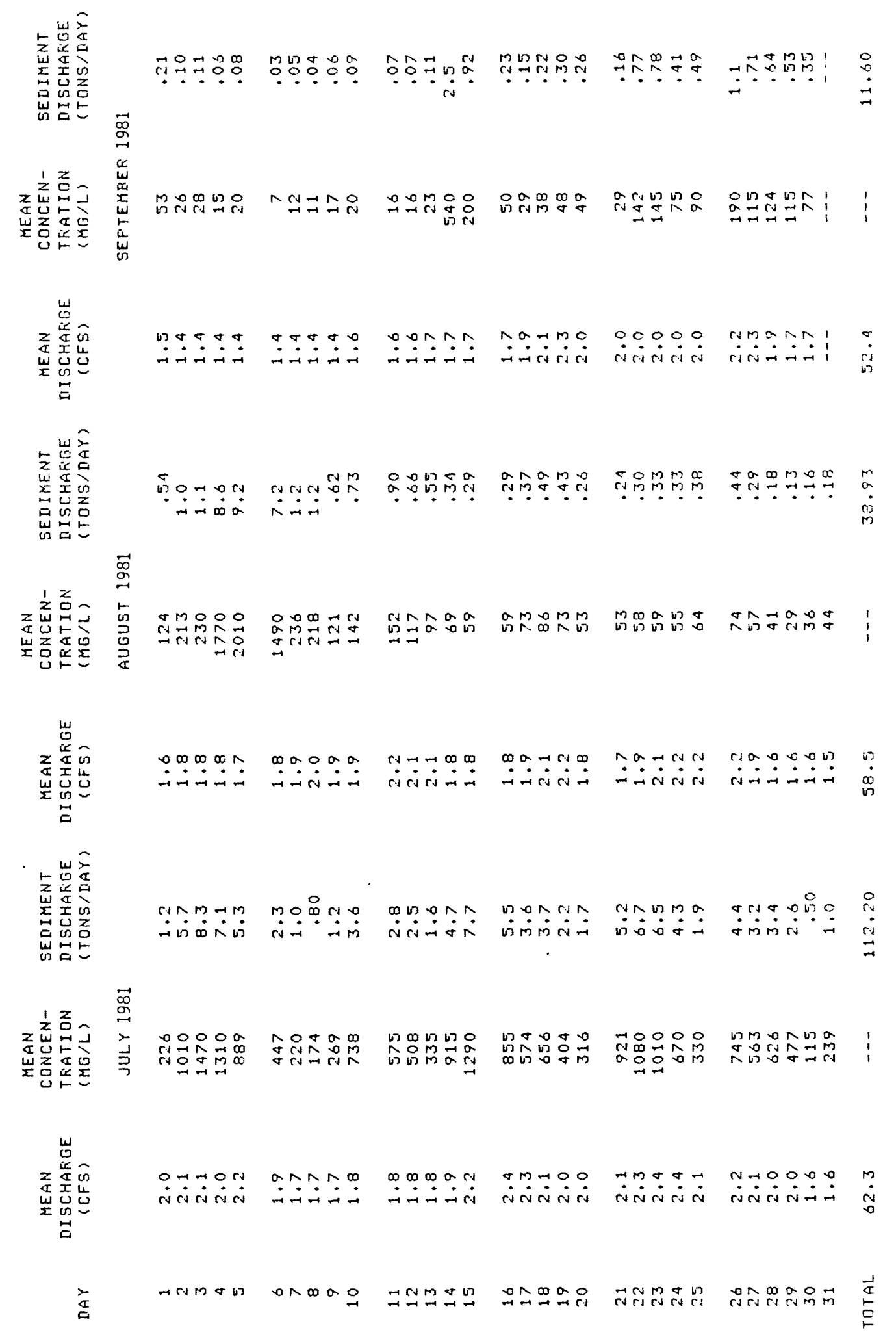




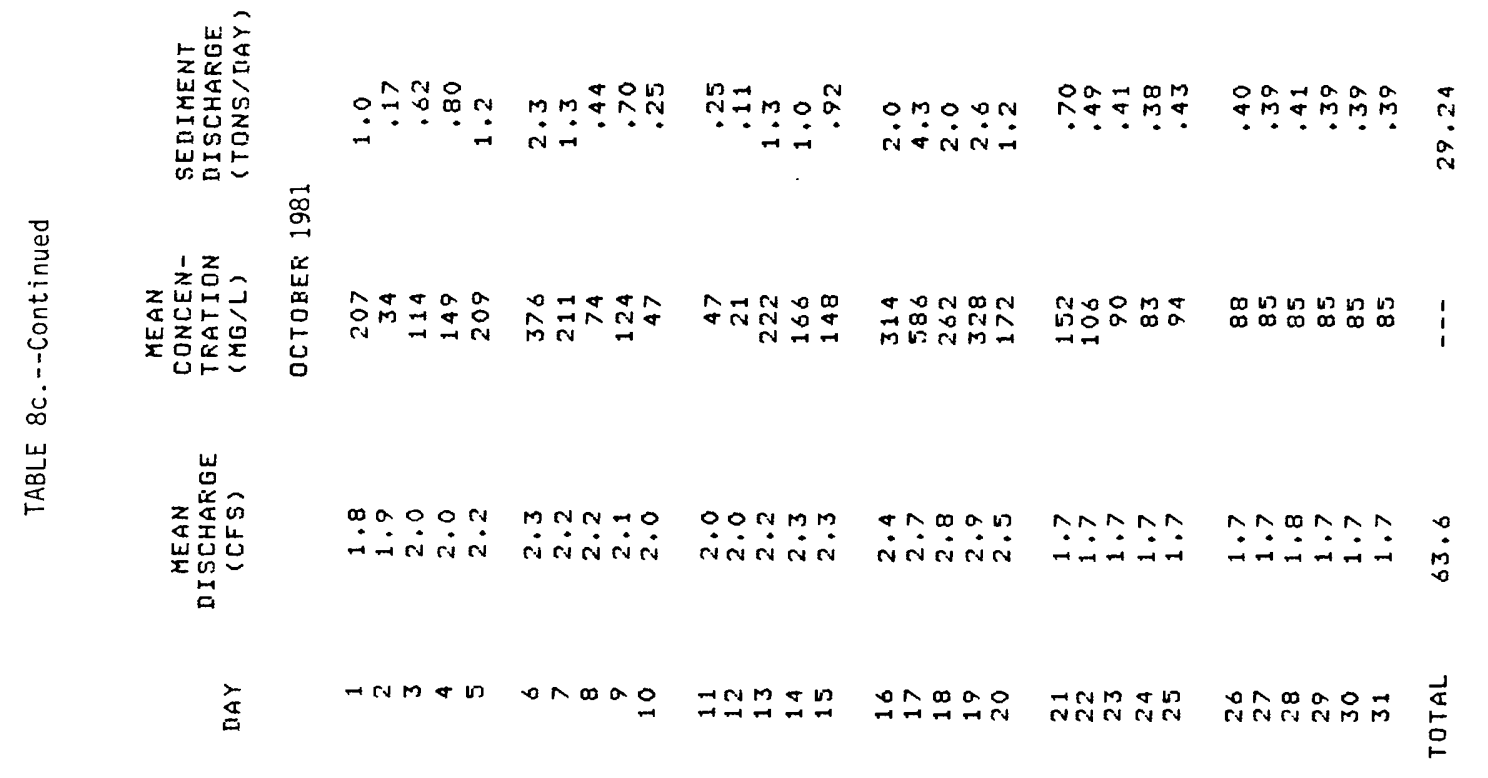




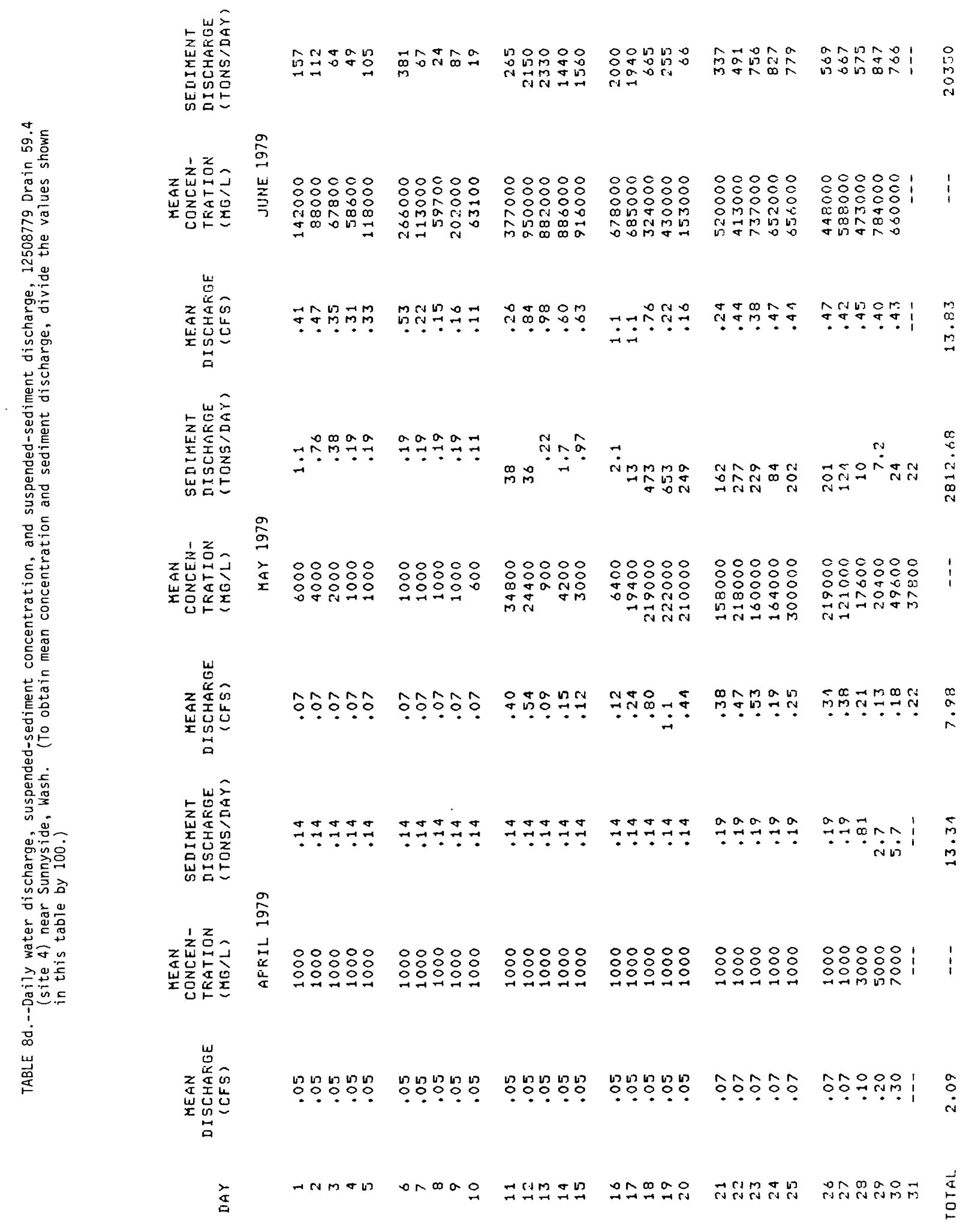




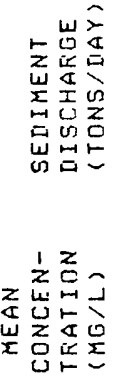

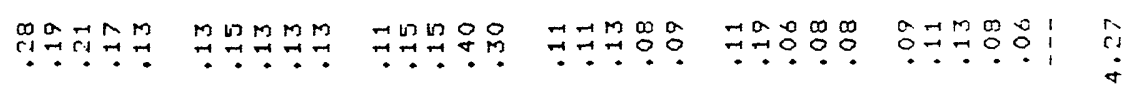

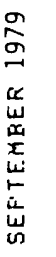

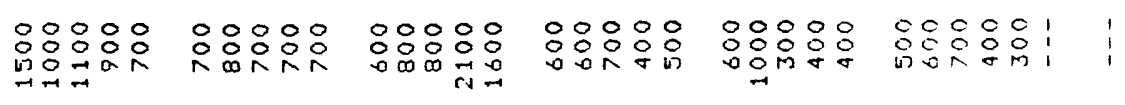

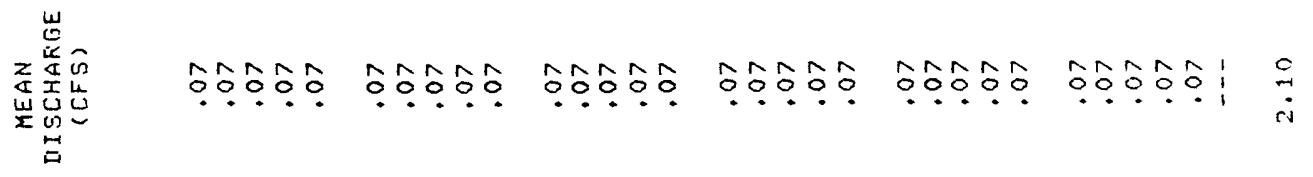

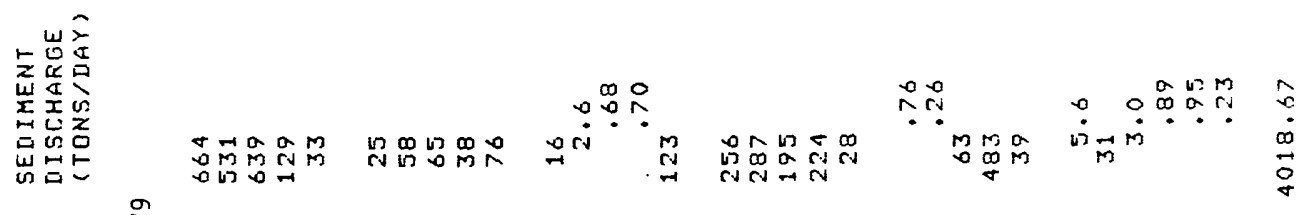

à

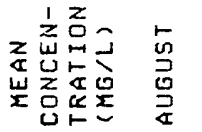

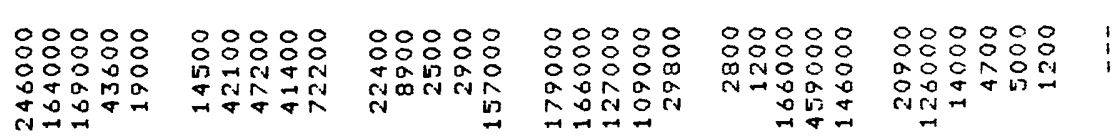

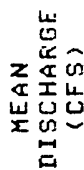

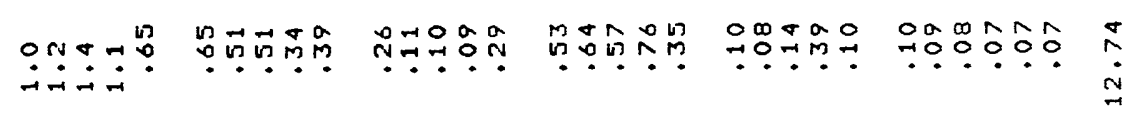

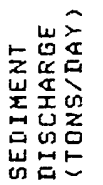

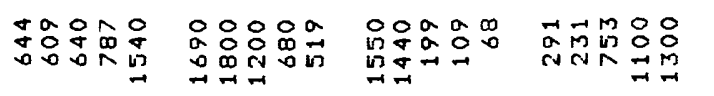

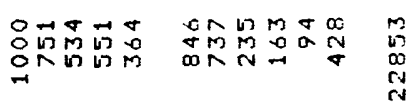

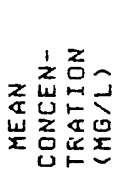

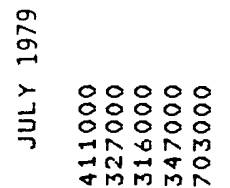

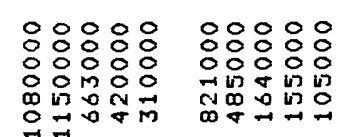

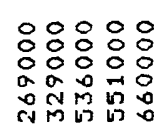

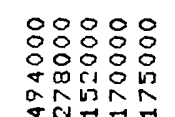

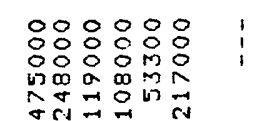

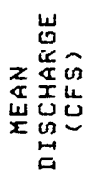

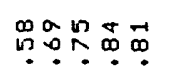

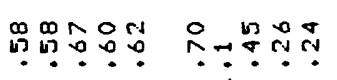

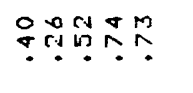

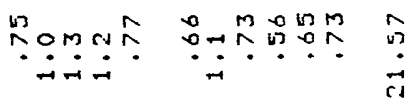

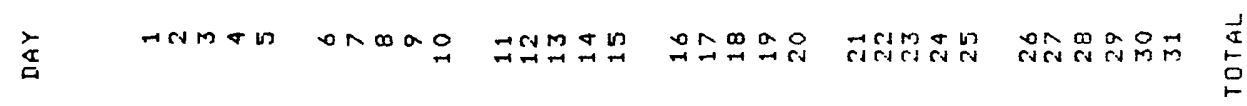




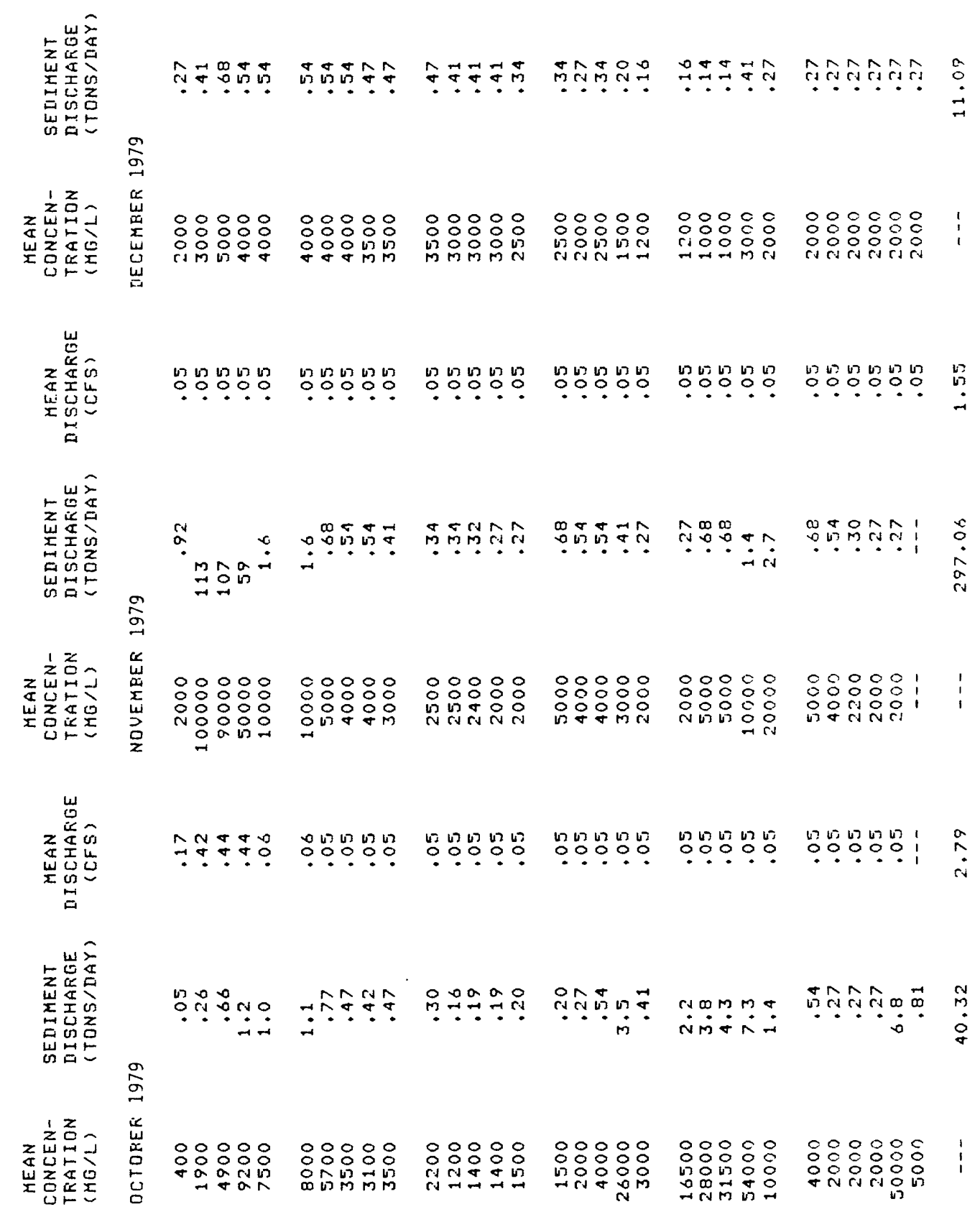

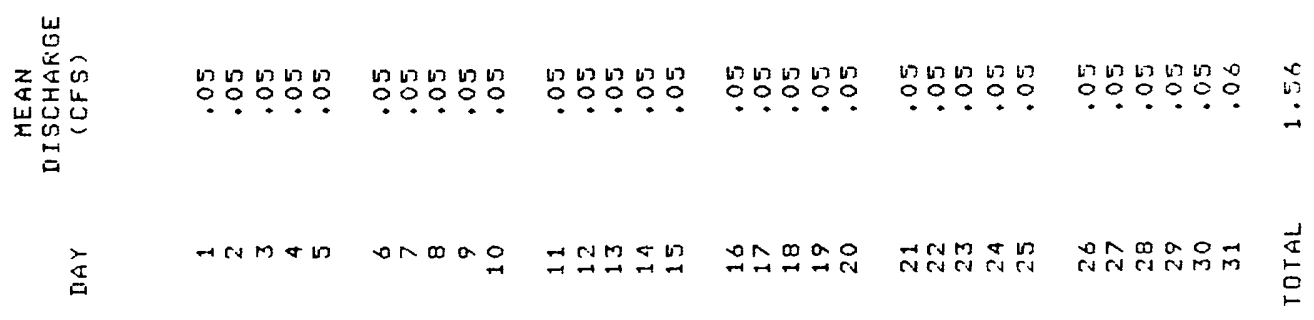




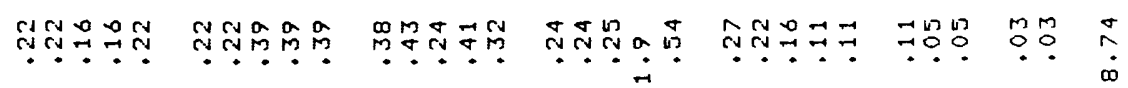

$\stackrel{\circ}{\stackrel{8}{g}}$

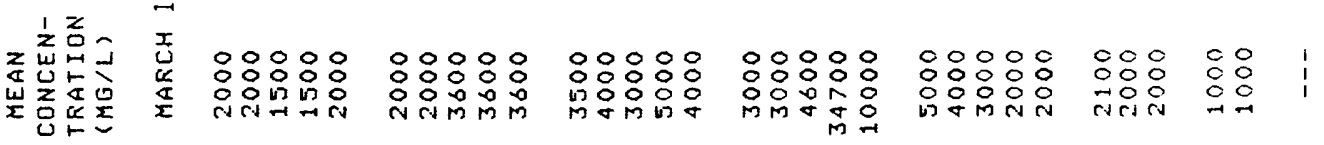

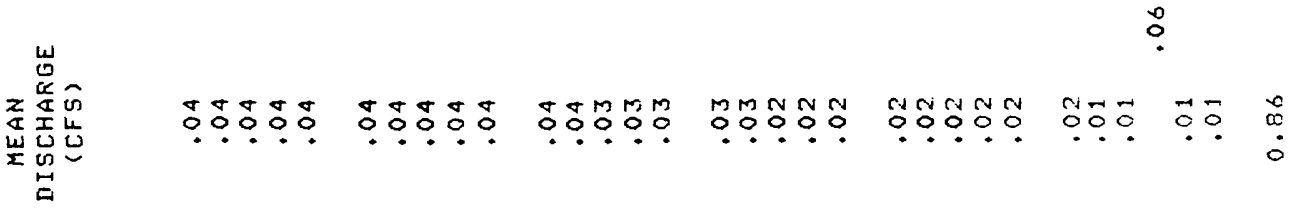

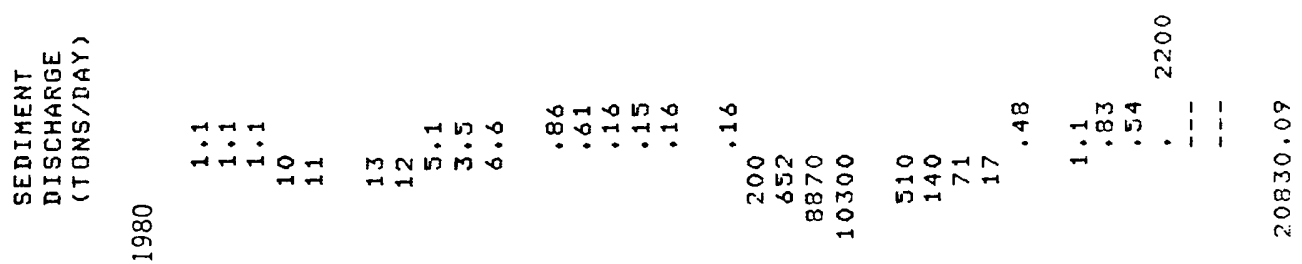

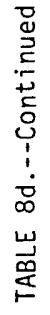

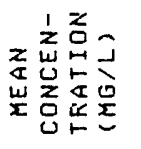

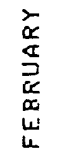

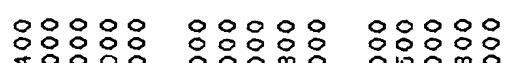

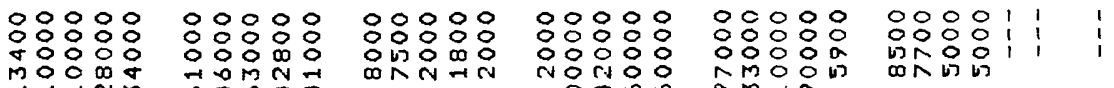

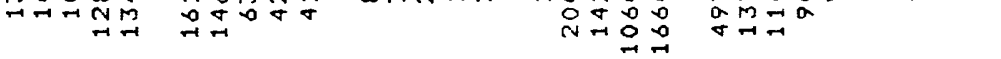

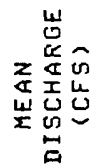

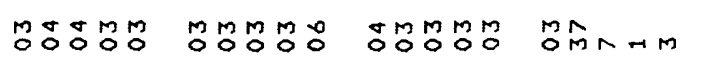

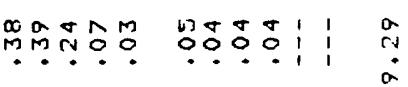

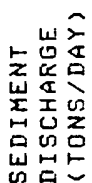

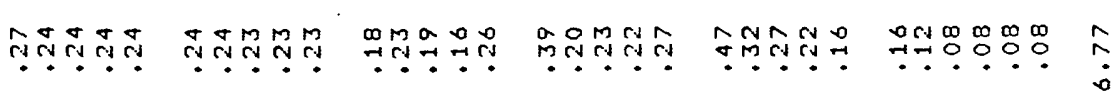

足

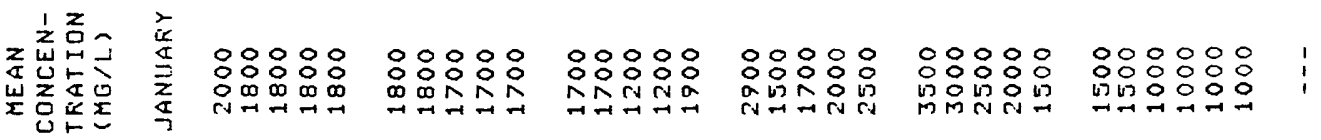

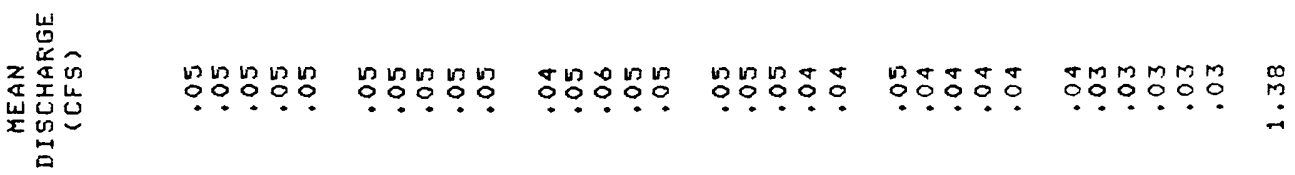

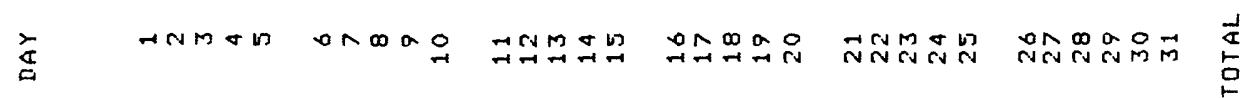




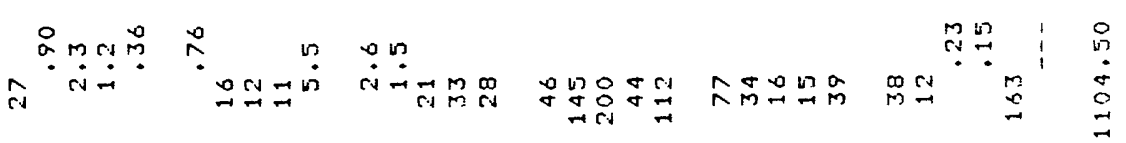

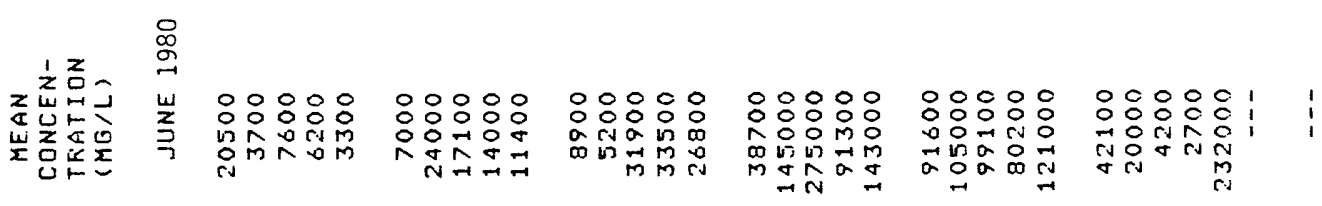

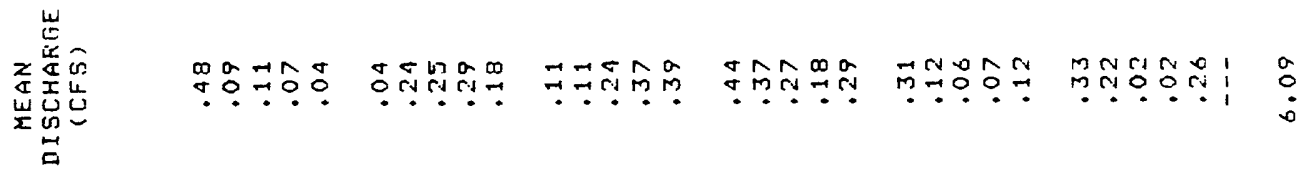

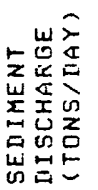

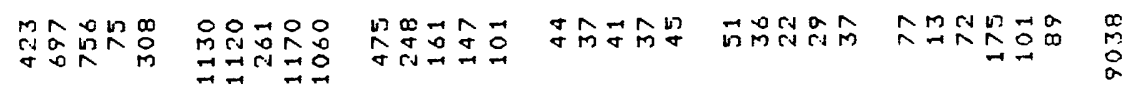

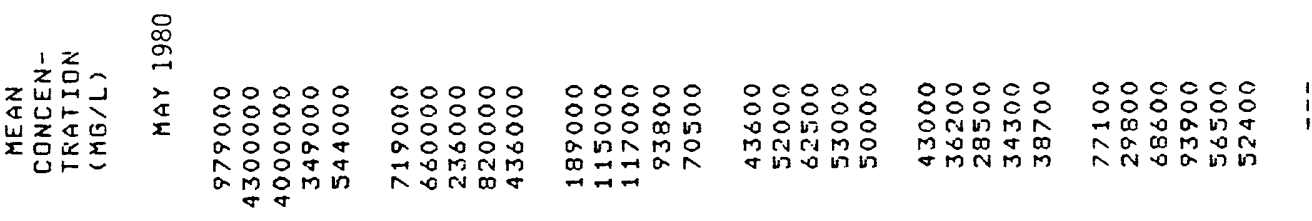
崖

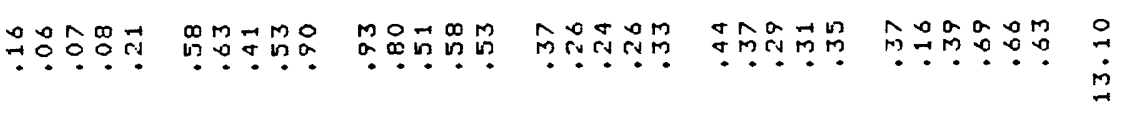

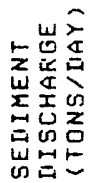

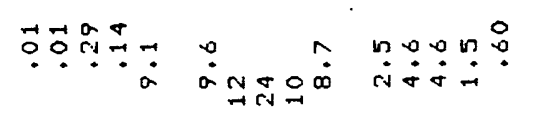

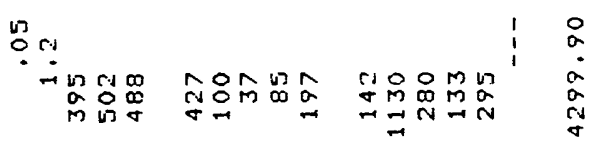
$\stackrel{\infty}{\circ}$

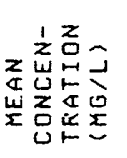

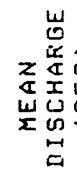

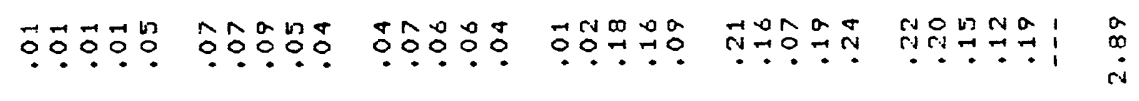

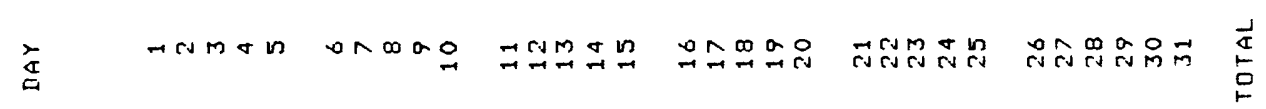




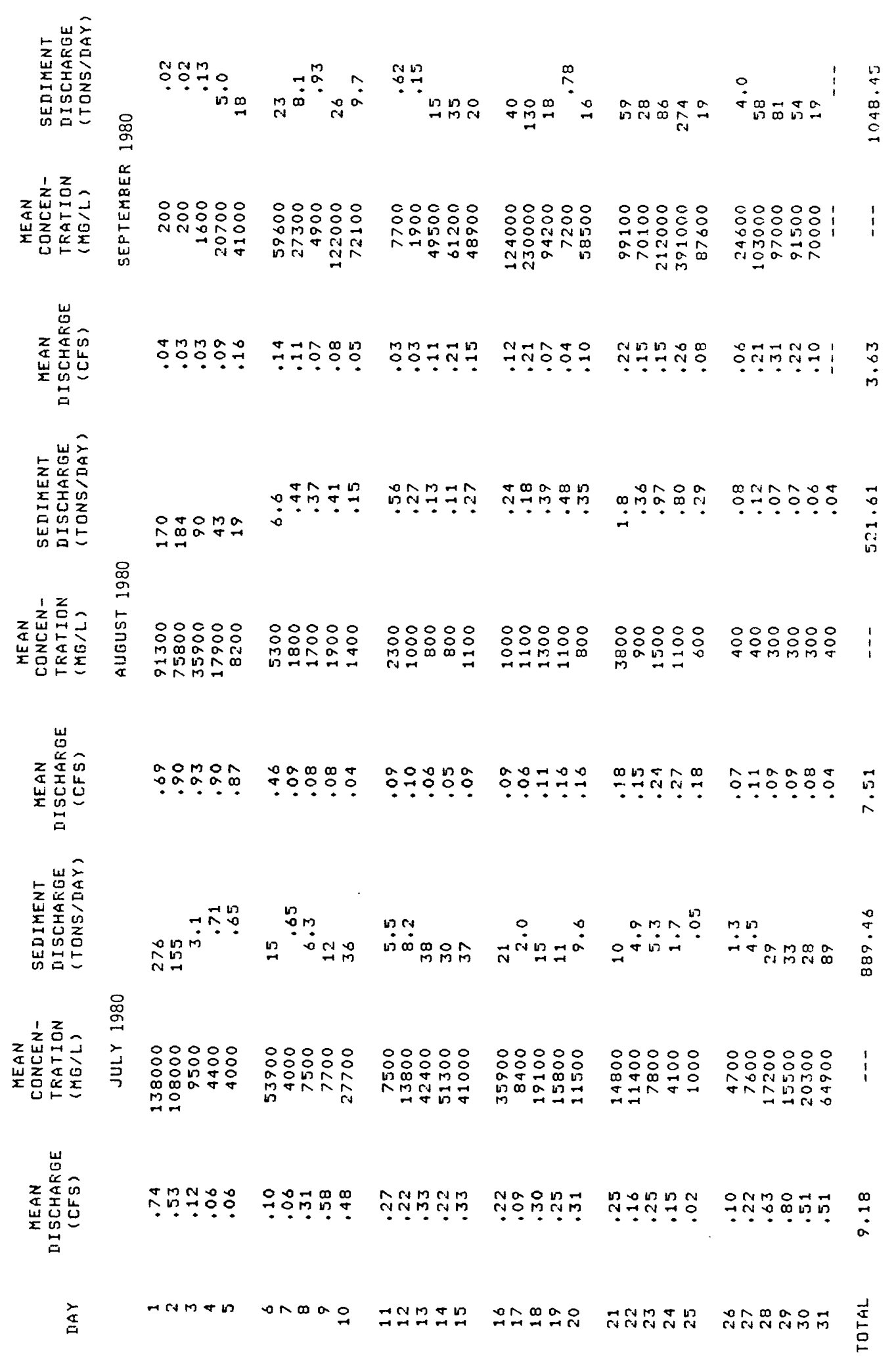




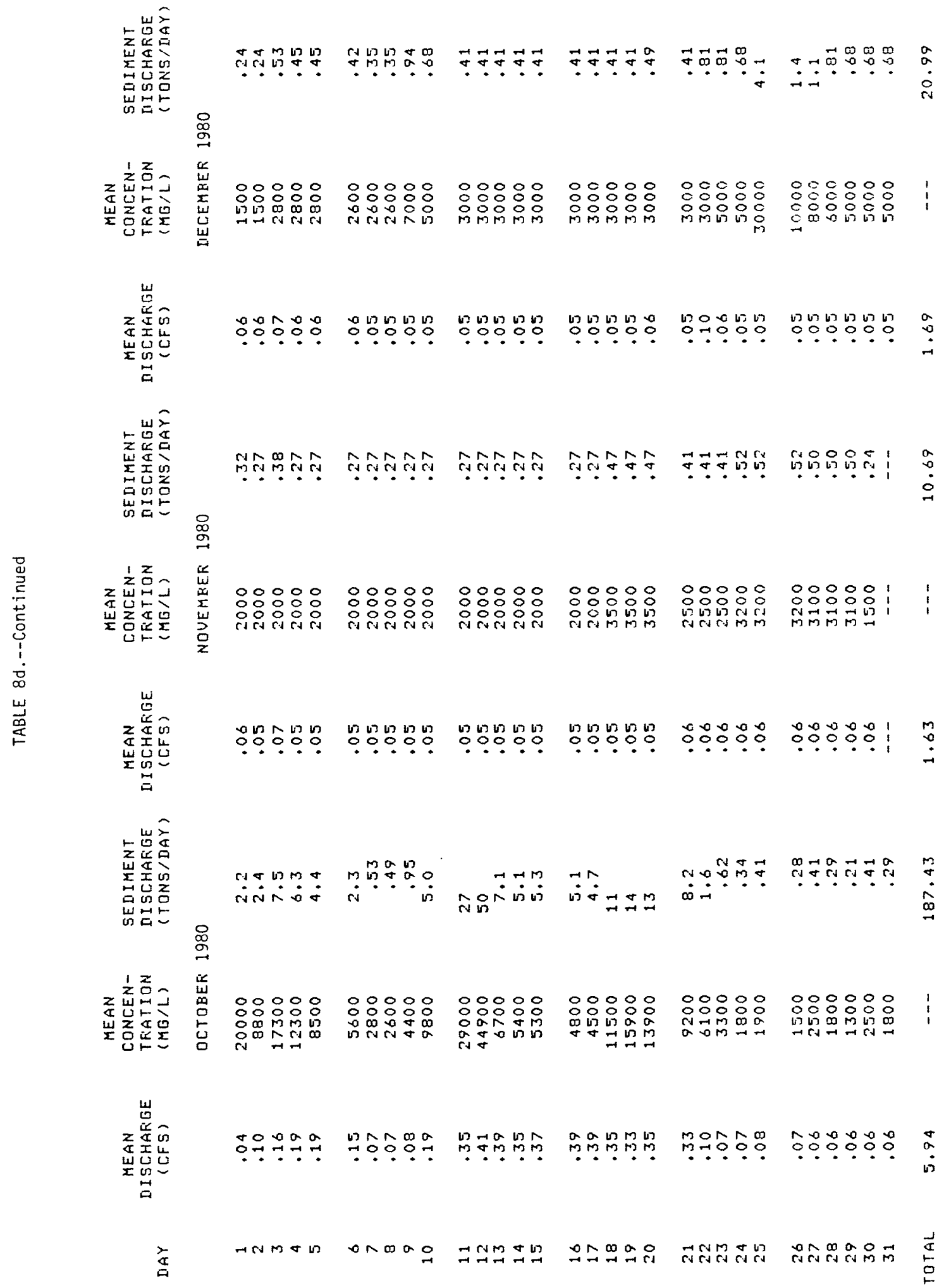




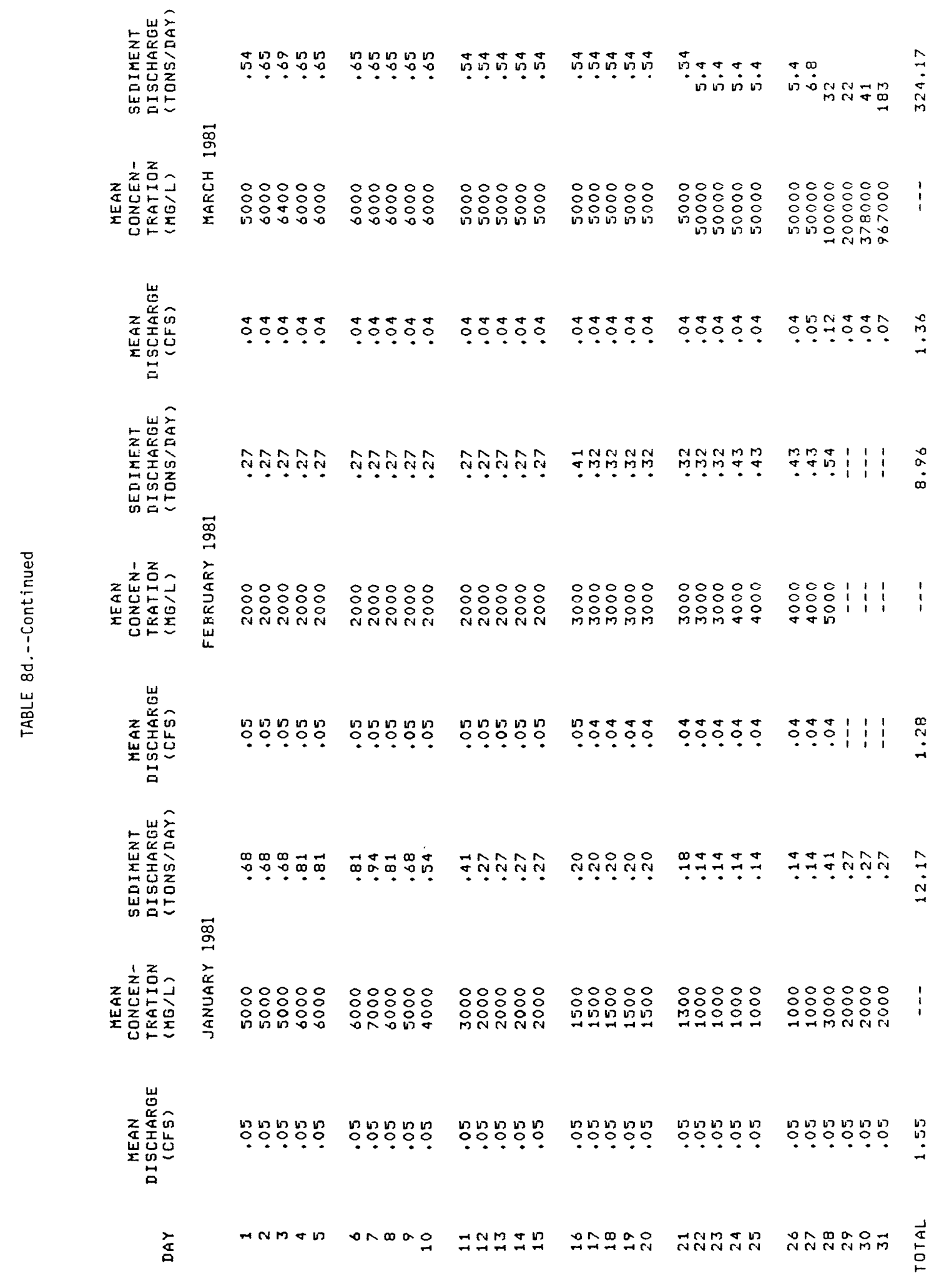




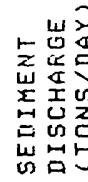

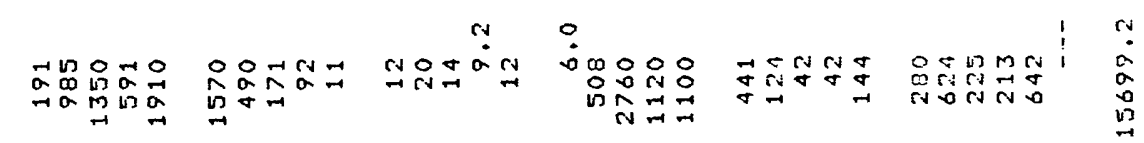

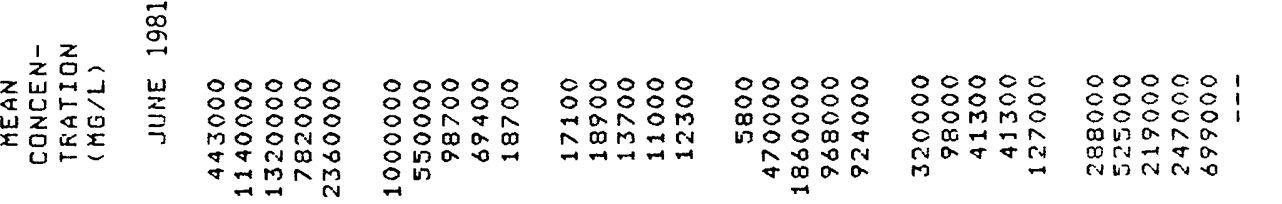

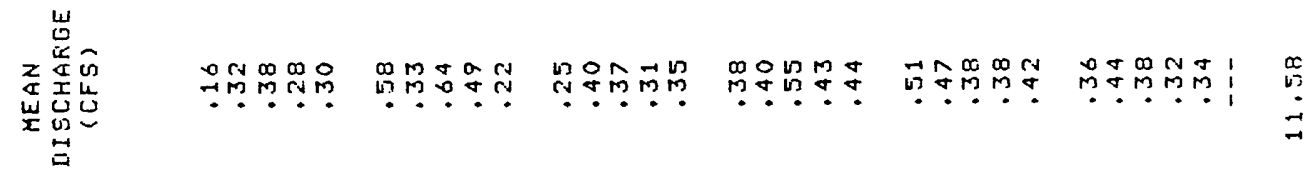

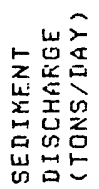

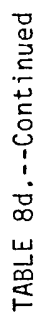

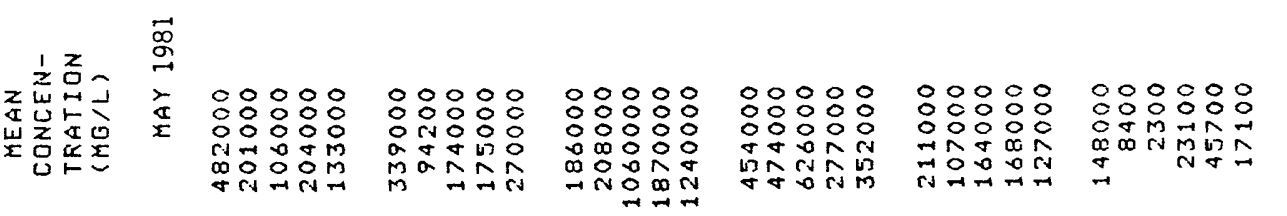

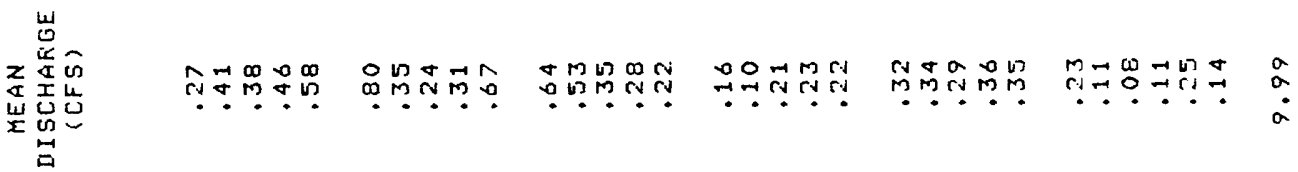

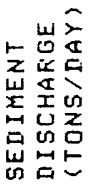

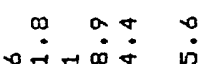

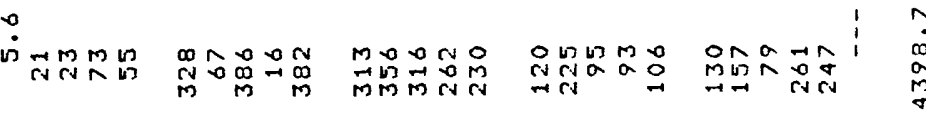

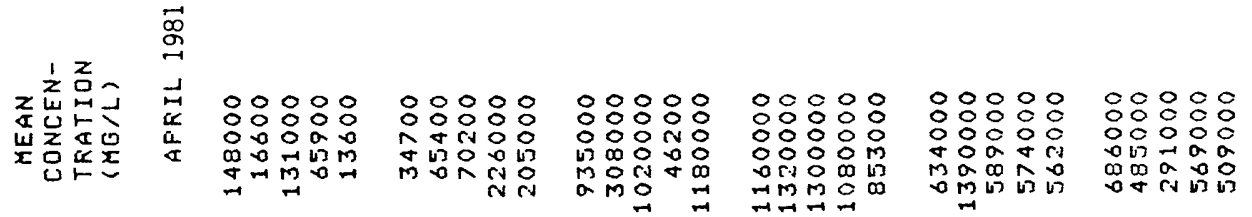

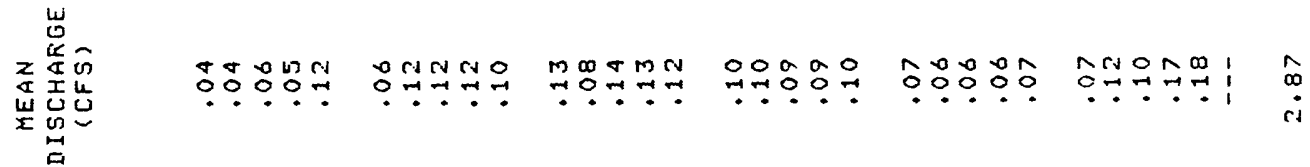

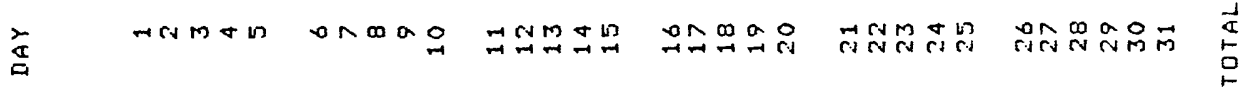




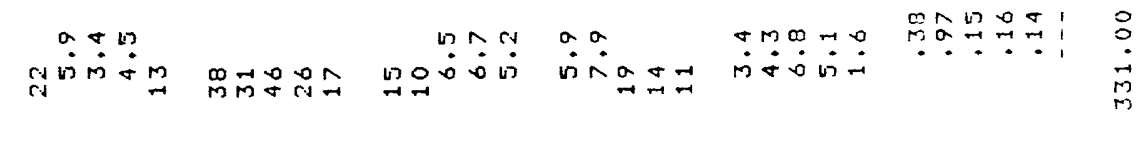

\section{$00: 0 \circ$}

\section{$\therefore \circ 000$}

응ㅇㅇㅇㅇ

$\therefore: 0: 0$

$\therefore: 0: 0$

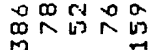

minn

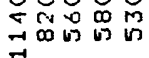

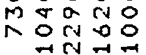

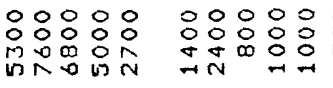

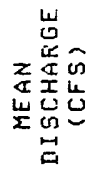

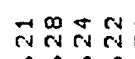

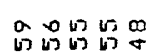

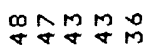

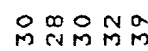

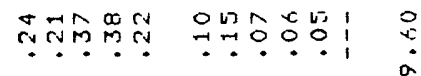

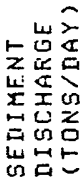

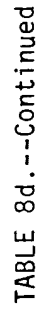

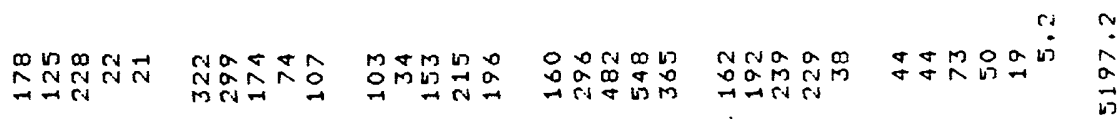

总

恙总

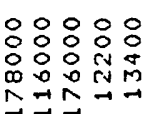

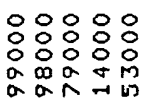

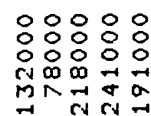

유요

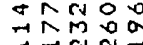

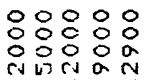

$\therefore: \therefore:: \circ$

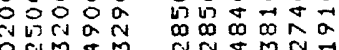

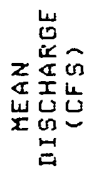

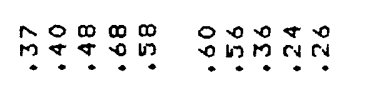

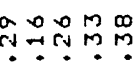

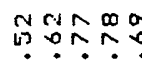

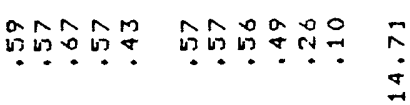

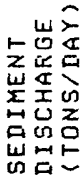

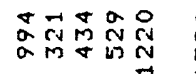

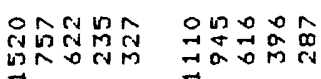

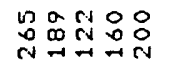

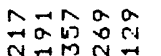

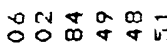

谙

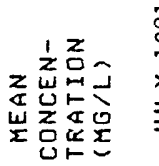

兽

خ

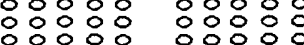

0

$\therefore: \therefore:$

m.

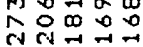

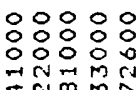

$\therefore: \therefore: ㅇ$

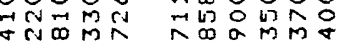

\section{$\bar{q}$}

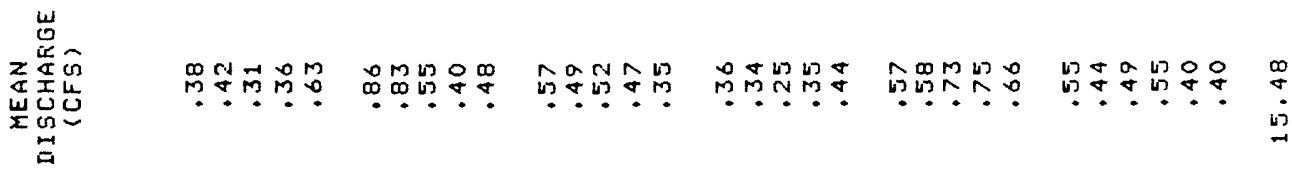

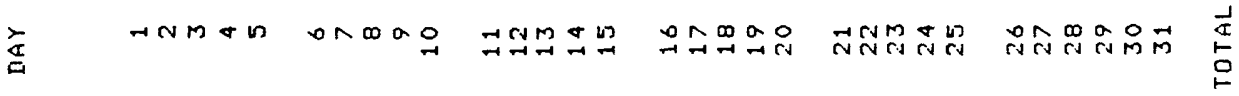




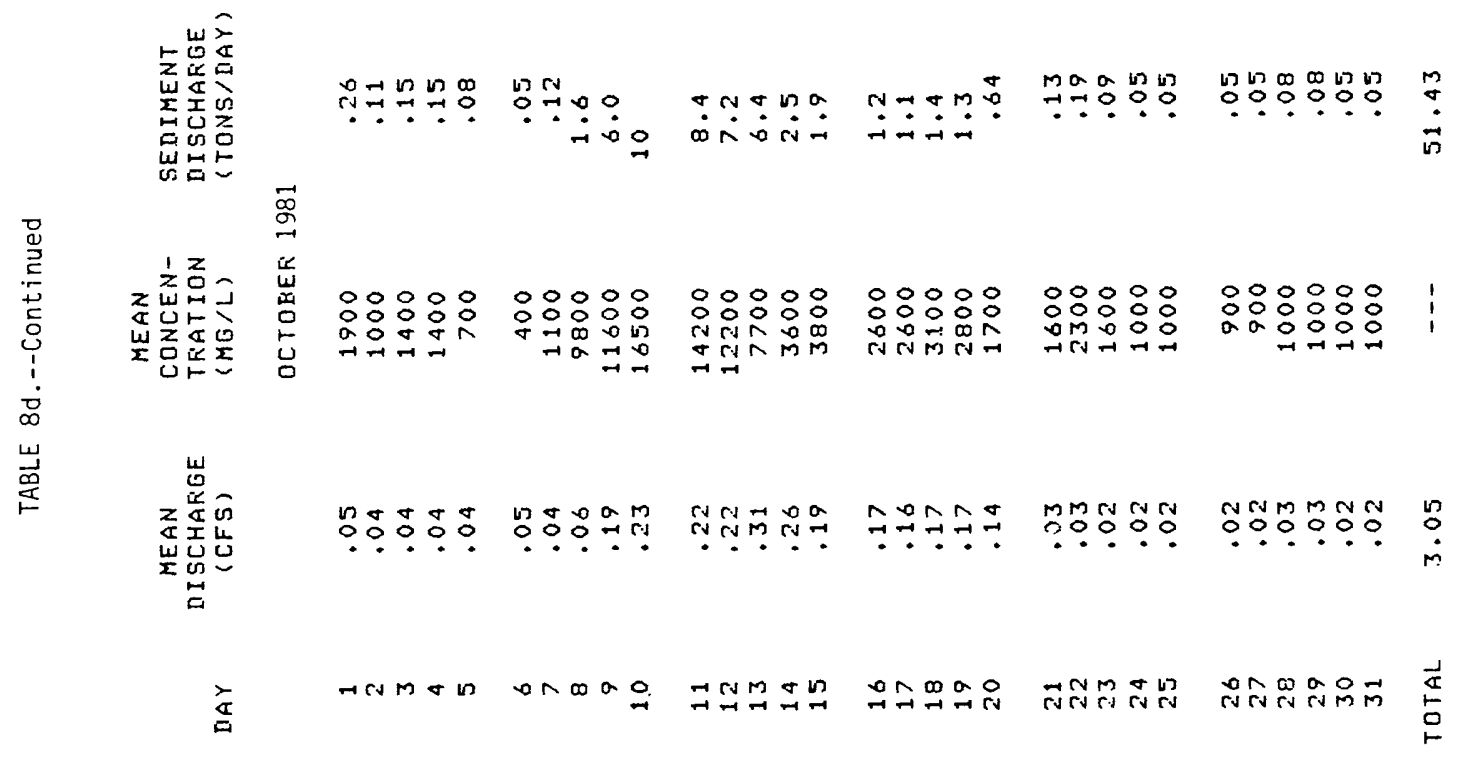


TABLE 9a.--Particle-size analysis of sediment for 12508775 Drain 61.0 (site 1) above Drain 61.4 near Sunnyside, Wash.

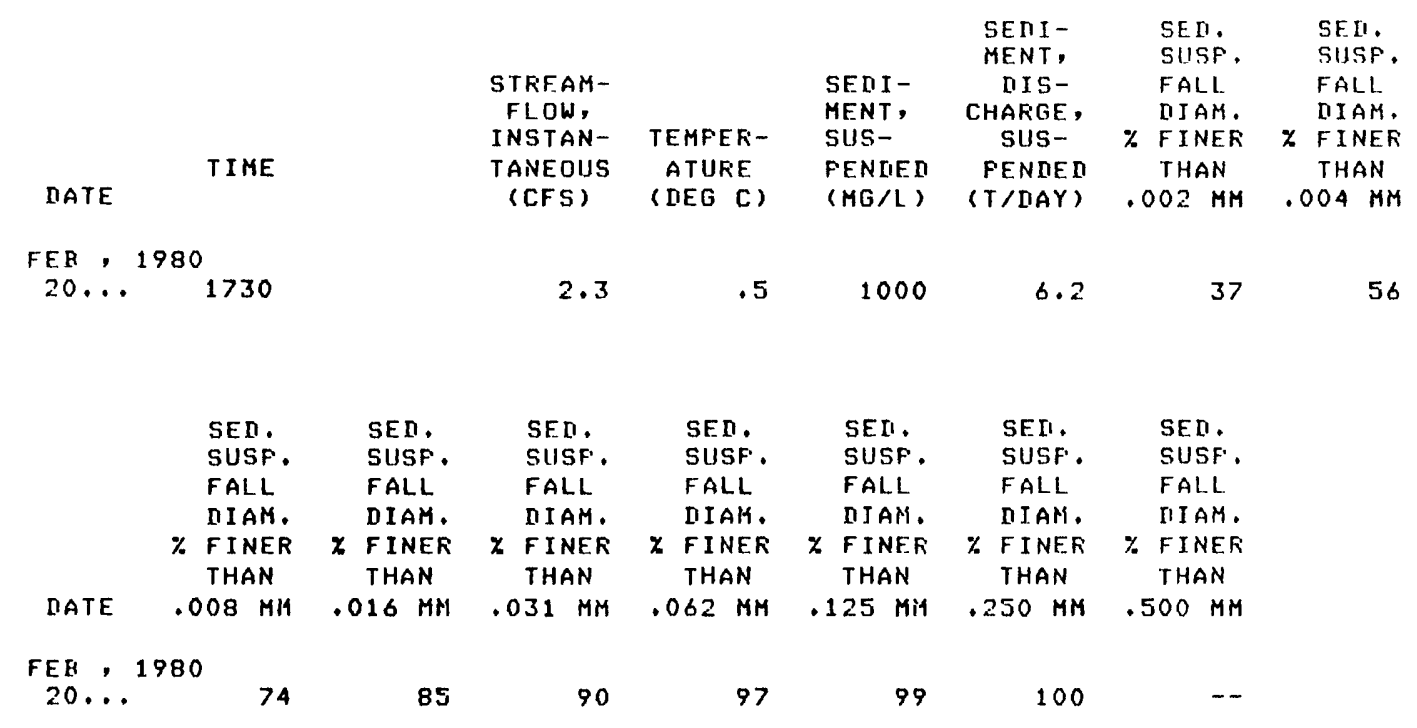

TABLE 9b.--Particle-size analysis of sediment for 12508769 Drain 60.7 (site 2) near Sunnyside, Wash.

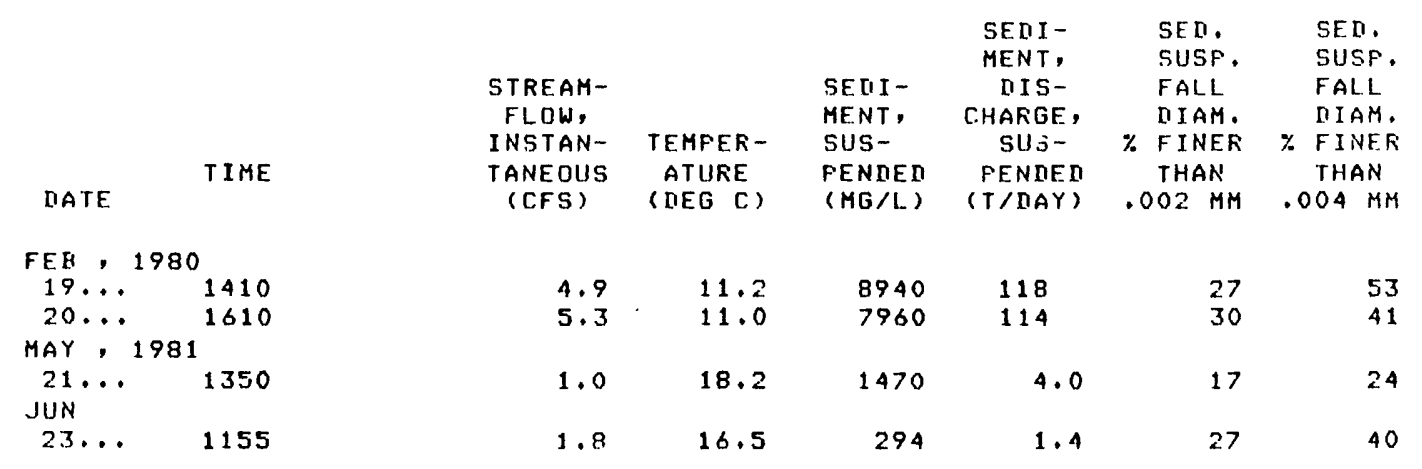

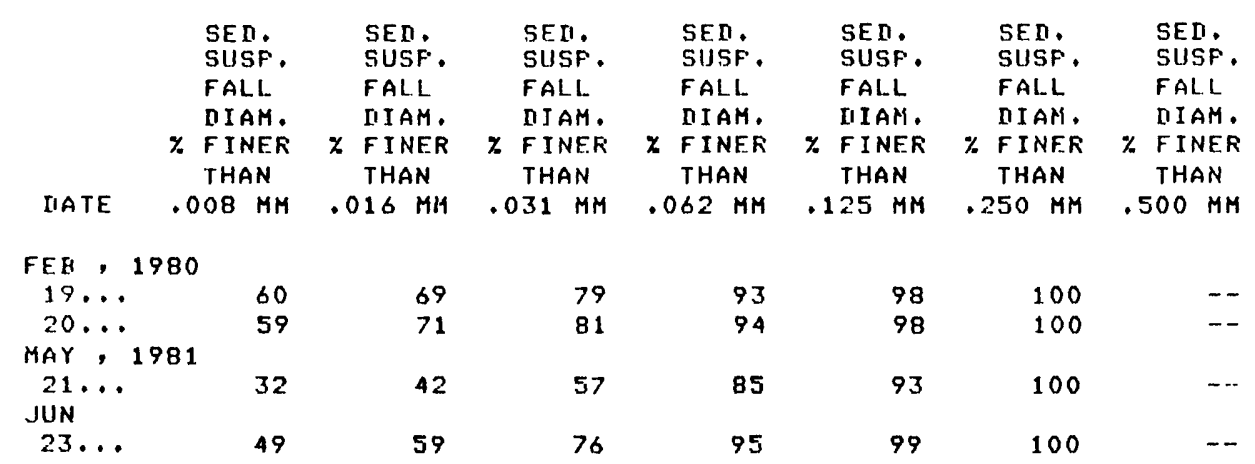


TABLE 9c.--Particle-size analysis of sediment for 12508775 Drain 59.6 (site 3) below Drain 60.2 near Sunnyside, Wash.

$\begin{array}{lr}\text { DATE } & \text { TIME } \\ \text { JUN , 1979 } \\ 01 \ldots & 1145 \\ 25 \ldots & 1150 \\ \text { FEB , } 1980 \\ 19 \ldots & 1325 \\ 20 \ldots & 1310 \\ \text { SEP } & \\ 04 \ldots & 1415 \\ 17 \ldots & 1055 \\ \text { JUN, } 1981 \\ 23 \ldots & 1420 \\ \text { JUL } & \\ 10 \ldots & 1110\end{array}$

$10 \ldots 1110$

\begin{tabular}{|c|c|c|c|c|c|}
\hline & & & $\begin{array}{l}\text { SEIII- } \\
\text { MENT, }\end{array}$ & $\begin{array}{l}\text { SED. } \\
\text { SUSP. }\end{array}$ & $\begin{array}{l}\text { SED. } \\
\text { SUSF. }\end{array}$ \\
\hline $\begin{array}{l}\text { STREAM- } \\
\text { FLOW, } \\
\text { INSTAN- }\end{array}$ & TEMPER - & $\begin{array}{l}\text { SEIII- } \\
\text { MENT, } \\
\text { SUS- }\end{array}$ & $\begin{array}{l}\text { IIIS- } \\
\text { CHARGE, } \\
\text { SUS- }\end{array}$ & $\begin{array}{l}\text { FALL } \\
\text { IIIAN. } \\
\% \text { FINER }\end{array}$ & $\begin{aligned} & \text { FALL } \\
& \text { IIIAM. } \\
\% & \text { FINEF }\end{aligned}$ \\
\hline $\begin{array}{l}\text { TANEOUS } \\
\text { (CFS) }\end{array}$ & $\begin{array}{l}\text { ATURE } \\
\text { (DEG C) }\end{array}$ & $\begin{array}{l}\text { FENDEI } \\
\text { (MG/L) }\end{array}$ & $\begin{array}{l}\text { FENIIED } \\
(T / \text { IIYY) }\end{array}$ & $\begin{array}{c}\text { THAN } \\
.002 \mathrm{MM}\end{array}$ & $\begin{array}{l}\text { THAN } \\
.004 \mathrm{MM}\end{array}$ \\
\hline
\end{tabular}

$\begin{array}{rrrcrr}2.1 & 17.8 & 638 & 3.6 & 6 & 11 \\ 2.6 & 18.4 & 330 & 2.3 & 16 & 24 \\ 10 & 1.2 & 30800 & 832 & 13 & 23 \\ 11 & 4.0 & 44000 & 1310 & 3 & 10 \\ 1.9 & 15.0 & 135 & .69 & 20 & 21 \\ 2.1 & 15.0 & 666 & 3.8 & 9 & 12 \\ 1.7 & 16.7 & 322 & 1.5 & 26 & 32 \\ 1.8 & 20.1 & 844 & 4.1 & 24 & 36\end{array}$

SER. SER. SER, SEI. SEII. SED. SEI.

SUSP. SUSF, SUSF, SUSF, SUSP. SUSF, SUSF.

FALL FALL FALL FALL FALL FALL FALL

DIAM. DIAM. IIIAM. DIAM. IIIAM. IIIAM. IIIAM.

\% FINER \% FINER \% FINER \% FINER \% FINER \% FINER \% FINER

THAN THAN THAN THAN THAN THAN THAN

DATE .008 MM .016 MIH .031 MM .062 MM .125 MIH .250 MM .500 MM

JUN, 1979

\begin{tabular}{|c|c|c|c|c|c|c|c|}
\hline $01 \ldots$ & 16 & 25 & $4 \mathrm{E}$ & 75 & 97 & 100 & -- \\
\hline $25 \ldots$ & 38 & 50 & 62 & 82 & 96 & 99 & 100 \\
\hline $\mathrm{FEB}, 1980$ & & & & & & & \\
\hline $19 \ldots$ & 30 & 47 & 69 & 90 & 99 & 100 & -- \\
\hline$\underset{S E F}{20}$ & 24 & 46 & 61 & 94 & 99 & 100 & -- \\
\hline $04 \ldots$ & 24 & 30 & 35 & -- & -- & -- & -- \\
\hline $17 \ldots$ & 20 & 47 & 56 & 95 & 98 & 100 & -- \\
\hline JUN, 1981 & & & & & & & \\
\hline$\underset{\text { JUL }}{23} \cdots$ & 46 & 61 & 75 & 95 & 100 & -- & -- \\
\hline $10 \ldots$ & 59 & 76 & 93 & 99 & 100 & $-\infty$ & - \\
\hline
\end{tabular}


TABLE 9d.--Particle-size analysis of sediment for 12508779 Drain 59.4 (site 4) near Sunnyside, Wash.

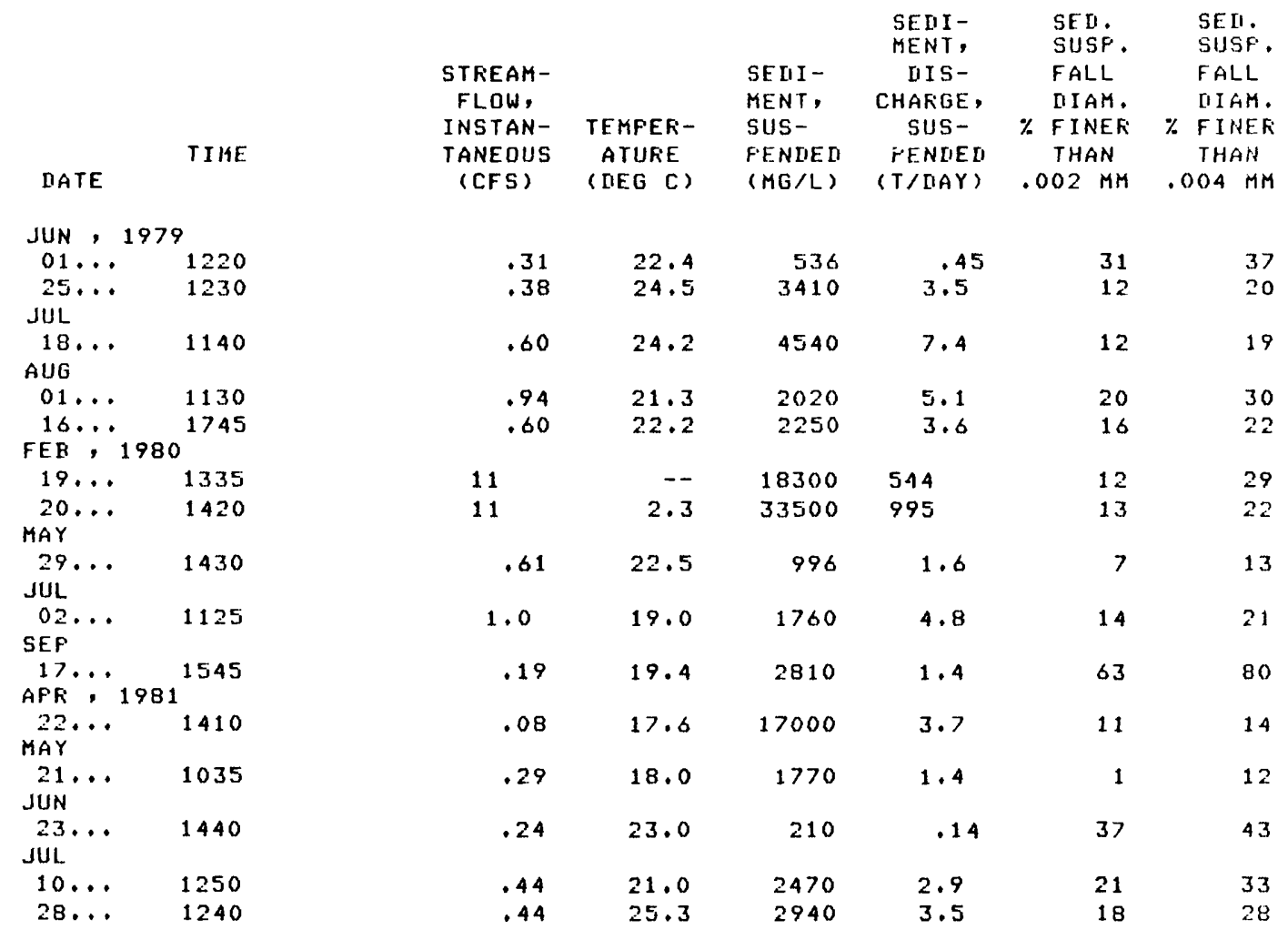

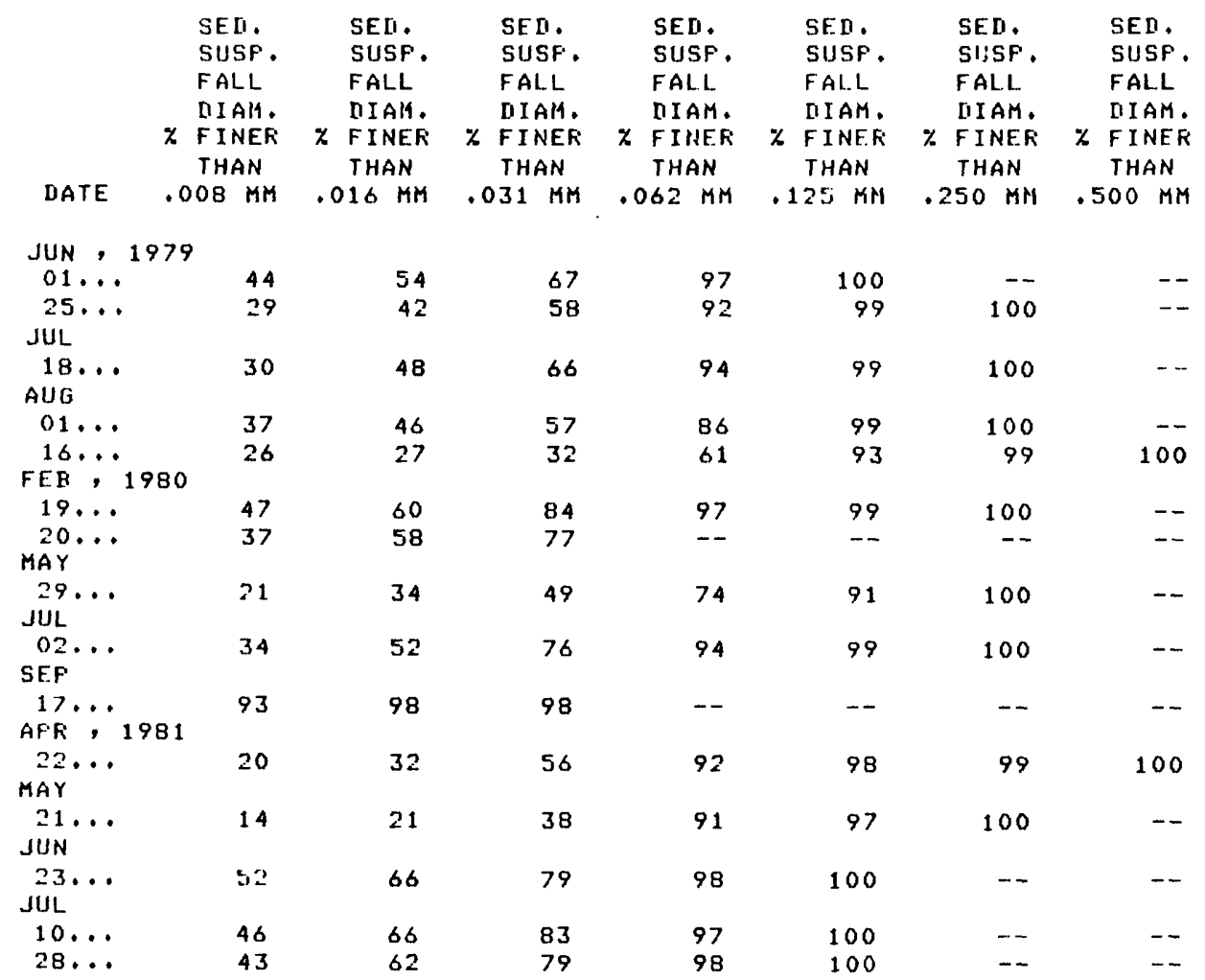




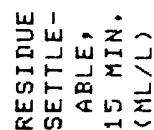

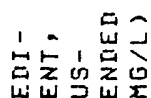

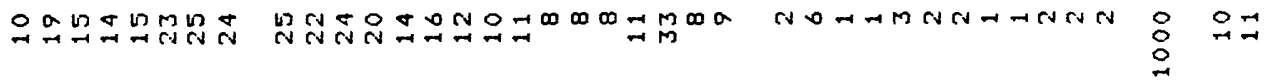

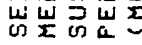

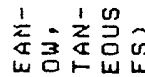

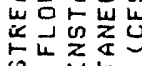

$\begin{array}{llllllll}1 & 1 & 1 & 1 & 1 & 1 & 1 & 1 \\ 1 & 1 & 1 & 1 & 1 & 1\end{array}$

$$
\begin{array}{lllllllllllllll}
1 & 1 & 1 & 1 & 1 & 1 & 1 & 1 & 1 & 1 & 1 & 1 & 1 & 1 & 1
\end{array}
$$$$
\begin{array}{llllllllllll}
1 & 1 & 1 & 1 & 1 & 1 & 1 & 1 & 1 & 1 & 1 & 1
\end{array}
$$$$
\text { 요 } \quad 1
$$

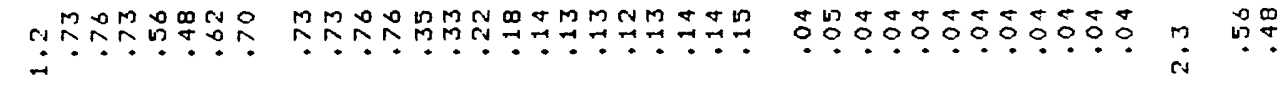

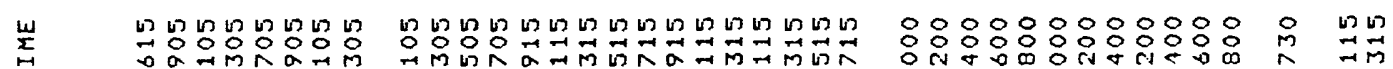

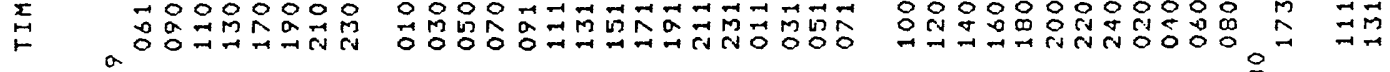
$a$

$\begin{array}{lllllll}1 & 1 & 1 & 1 & 1 & 1 & 1 \\ 1 & 1 & 1 & 1 & 1 & 1\end{array}$ $\begin{array}{lllllllllllllll}1 & 1 & 1 & 1 & 1 & 1 & 1 & 1 & 1 & 1 & 1 & 1 & 1 & 1 & 1\end{array}$ $\begin{array}{lllllllllllllll}1 & 1 & 1 & 1 & 1 & 1 & 1 & 1 & 1 & 1 & 1 & 1 & 0 & 1 & 1\end{array}$

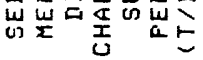

\section{崖它的家}

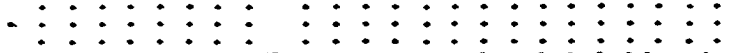

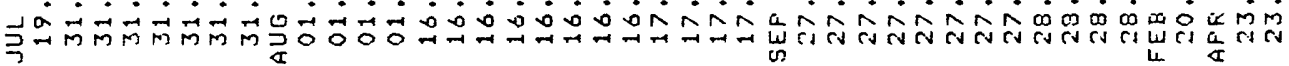

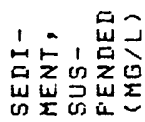

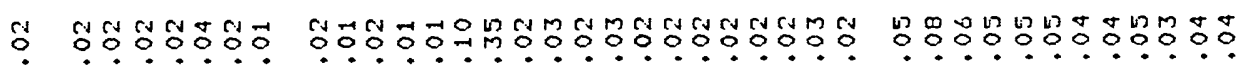

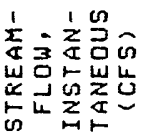

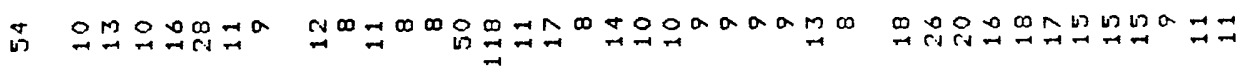

1 1 1 1 1001

:

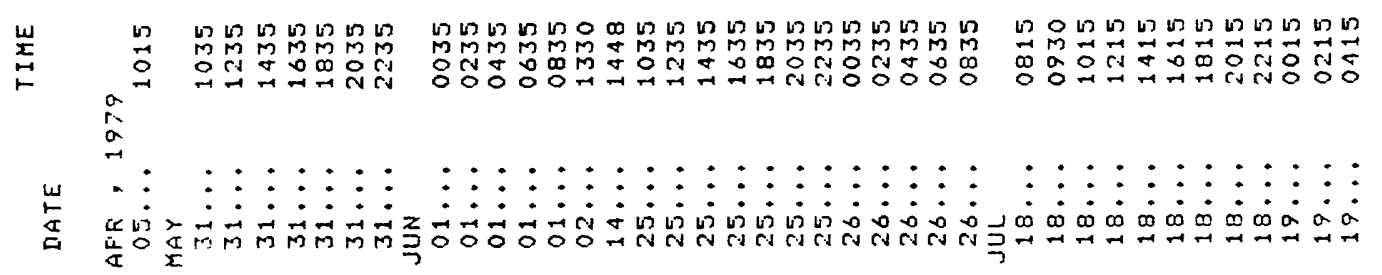




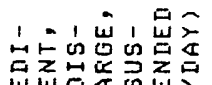

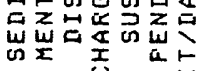

岂㟧。

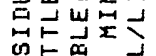

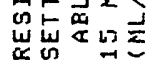

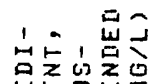

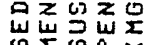

交文文合的 w正占岕

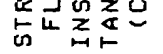

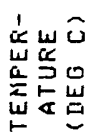

$\underset{5}{\stackrel{w}{x}}$

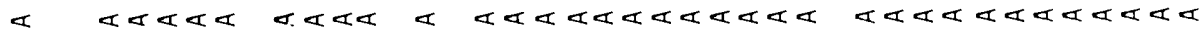

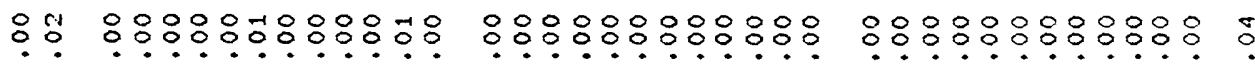

m

어요

mathoorroomom

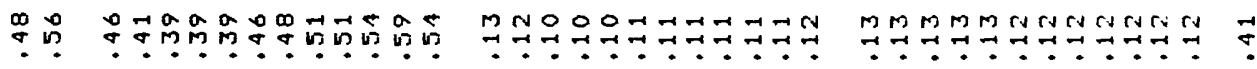

$\begin{array}{llllllllllllll}1 & 1 & 1 & 1 & 1 & 1 & 1 & 1 & 1 & 1 & 1 & 1 & 1\end{array}$

$\begin{array}{lllllllllll}1 & 1 & 1 & 1 & 1 & 1 & 1 & 1 & 1 & 1 & 1\end{array}$

$\begin{array}{lllllllllllll}1 & 1 & 1 & 1 & 1 & 1 & 1 & 1 & 1 & 1 & 1 & 1 & 1\end{array}$

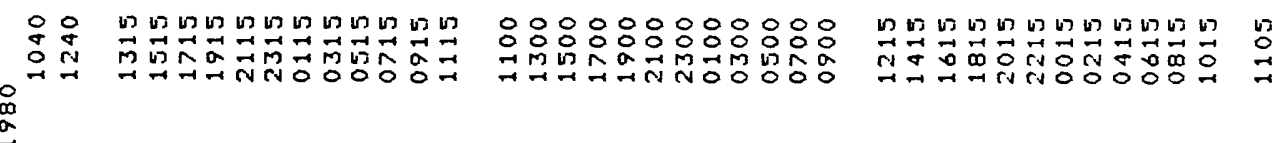

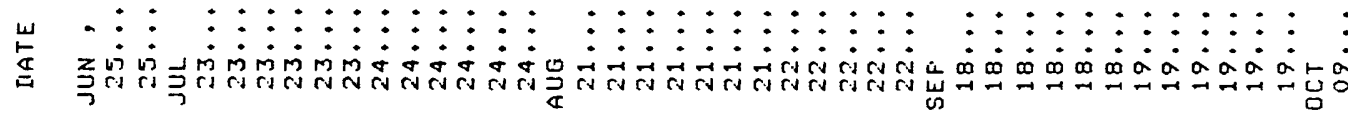

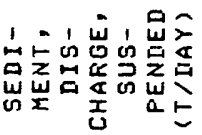

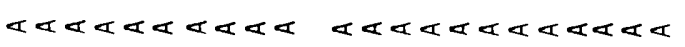

$\ll<\ll<$

$\varangle<$

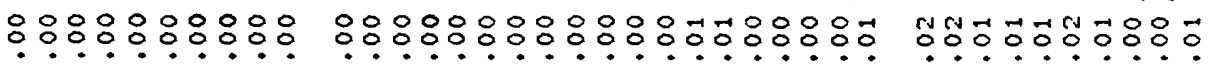

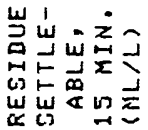

$\begin{array}{lllllllllllllllllllllllllllll}1 & 1 & 1 & 1 & 1 & 1 & 1 & 1 & 1 & 1 & 0 & 1 & 1 & 1 & 1 & 1 & 1 & 1 & 1 & 1 & 1 & 1 & 1 & 1 & 1 & 1 & 1 & 1 & 1\end{array}$

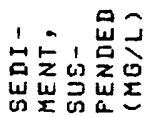

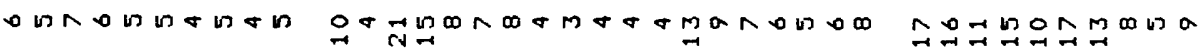

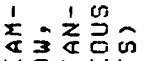

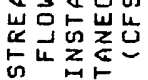

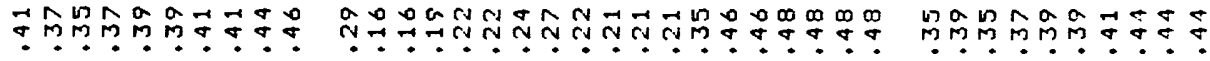

它

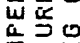

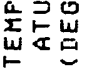

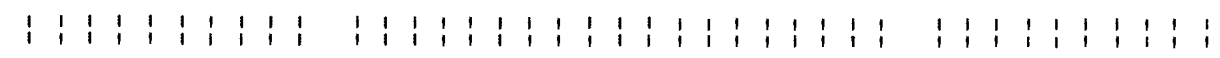

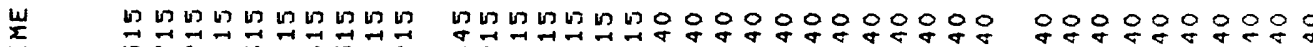

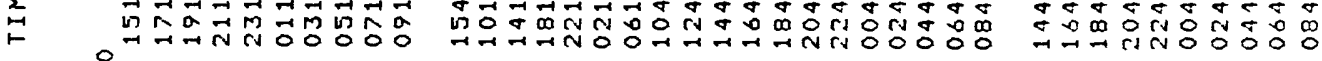
$\underset{\substack{\infty \\-1}}{\infty}$

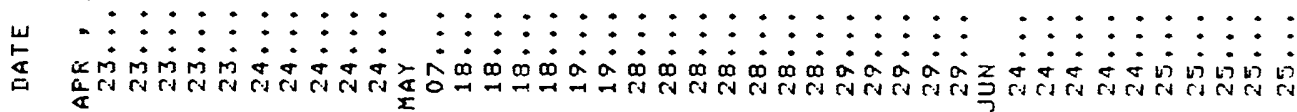


$\alpha<<<<<\alpha<\alpha<<<<<<$

$\alpha<\alpha<<<<<<<<<<<<<<$

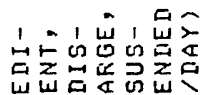

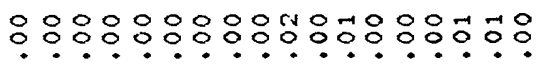

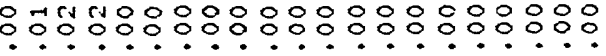

wi

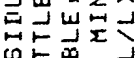

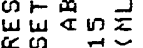

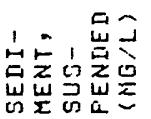

$\sum_{\frac{1}{3}}=\frac{1}{2} \frac{\omega}{\partial} \hat{0}$

w。

G

I

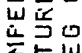

崖点岂

$\stackrel{w}{\leftarrow}$

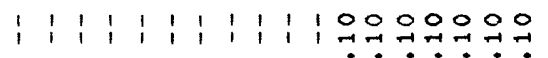 \\ $\dot{\forall} \dot{\forall} \dot{\forall} \dot{v}$}

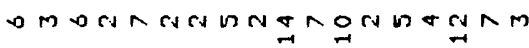

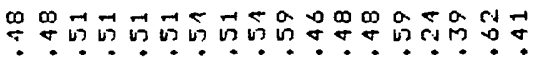

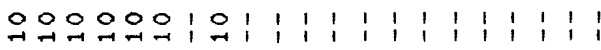

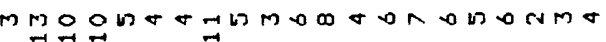
$\forall \dot{\forall} \dot{\forall} \dot{\forall}$

måำ

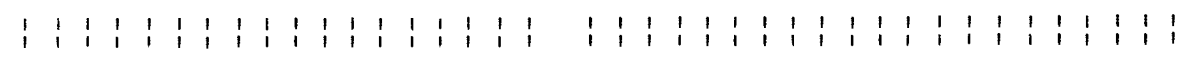

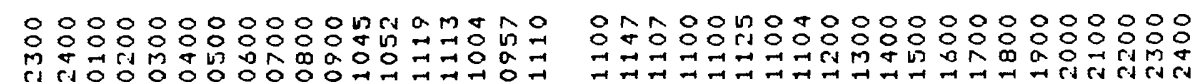
$\overrightarrow{0}$

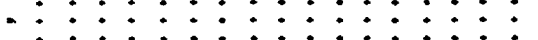

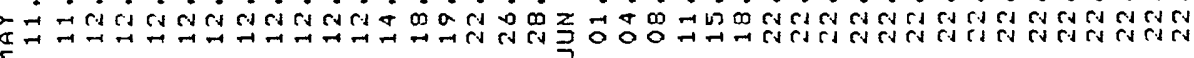

$x<x<<<<<<<<$

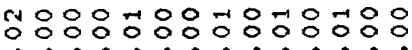

எ. $\varangle<\quad<<<<$ $\therefore 0: 0 \div 0: 0: 0$ $<\quad<\quad<\ll<\pi \quad<\ll<\alpha$ $\because 00000: 00: 00: 000$

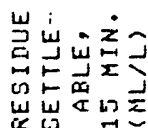

元, 岂今

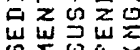

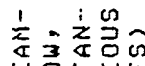

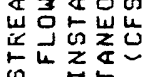

$\dot{1} w \hat{0}$

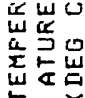

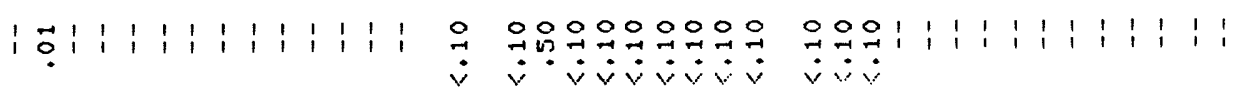

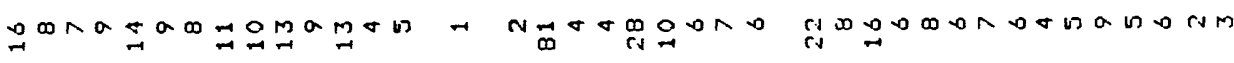

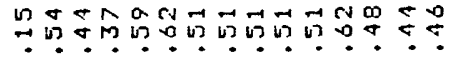

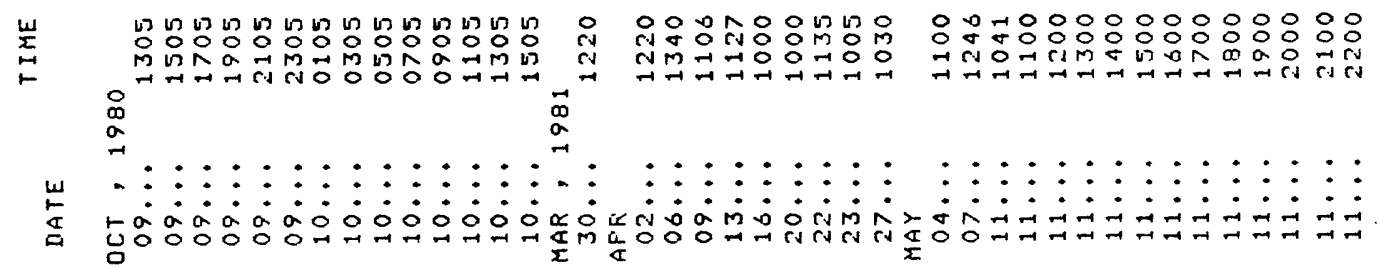




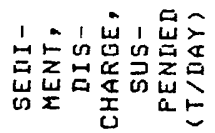

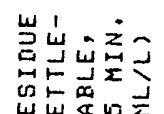

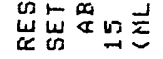

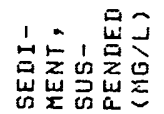

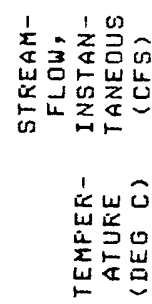

$0 ㅇ ㅇ ㅇ ㅇ$

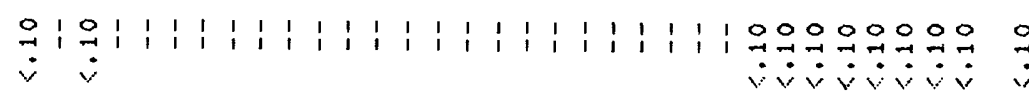

$\mathbb{M}_{\substack{0 \\ \hdashline} \rightarrow \infty}^{\infty}$

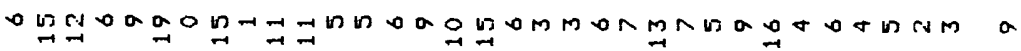

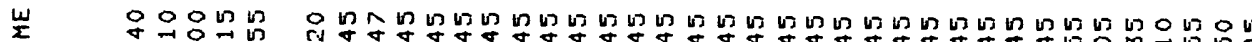

点

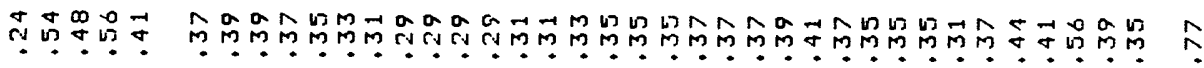
$\begin{array}{llll}1 & 1 & 1 & 1 \\ 1 & 1 & 1 & 1\end{array}$ i 1 $\exists=$

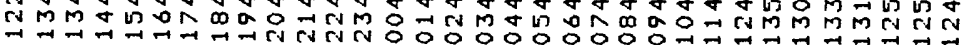
a

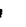

$: \dot{0}:: 0$

J

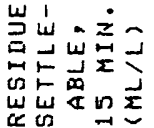

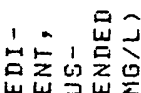

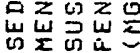

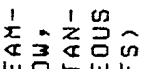

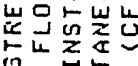

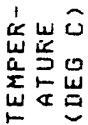

$<<<<<<<<<<$

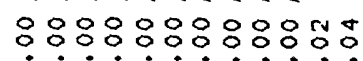

$\varangle$

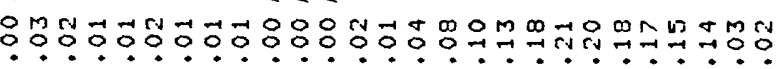

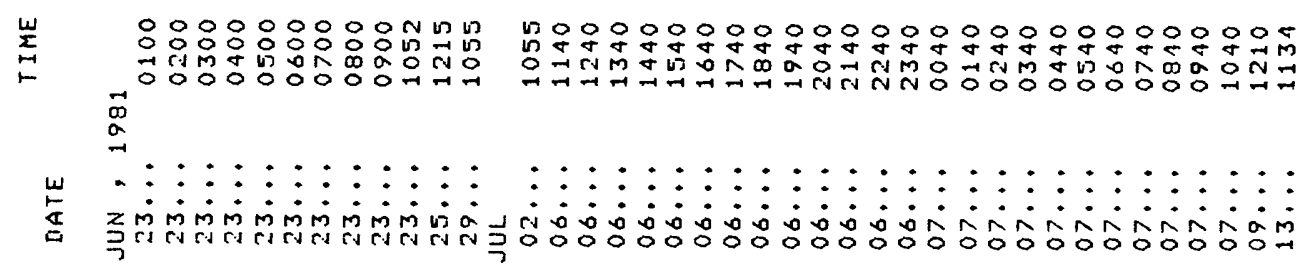




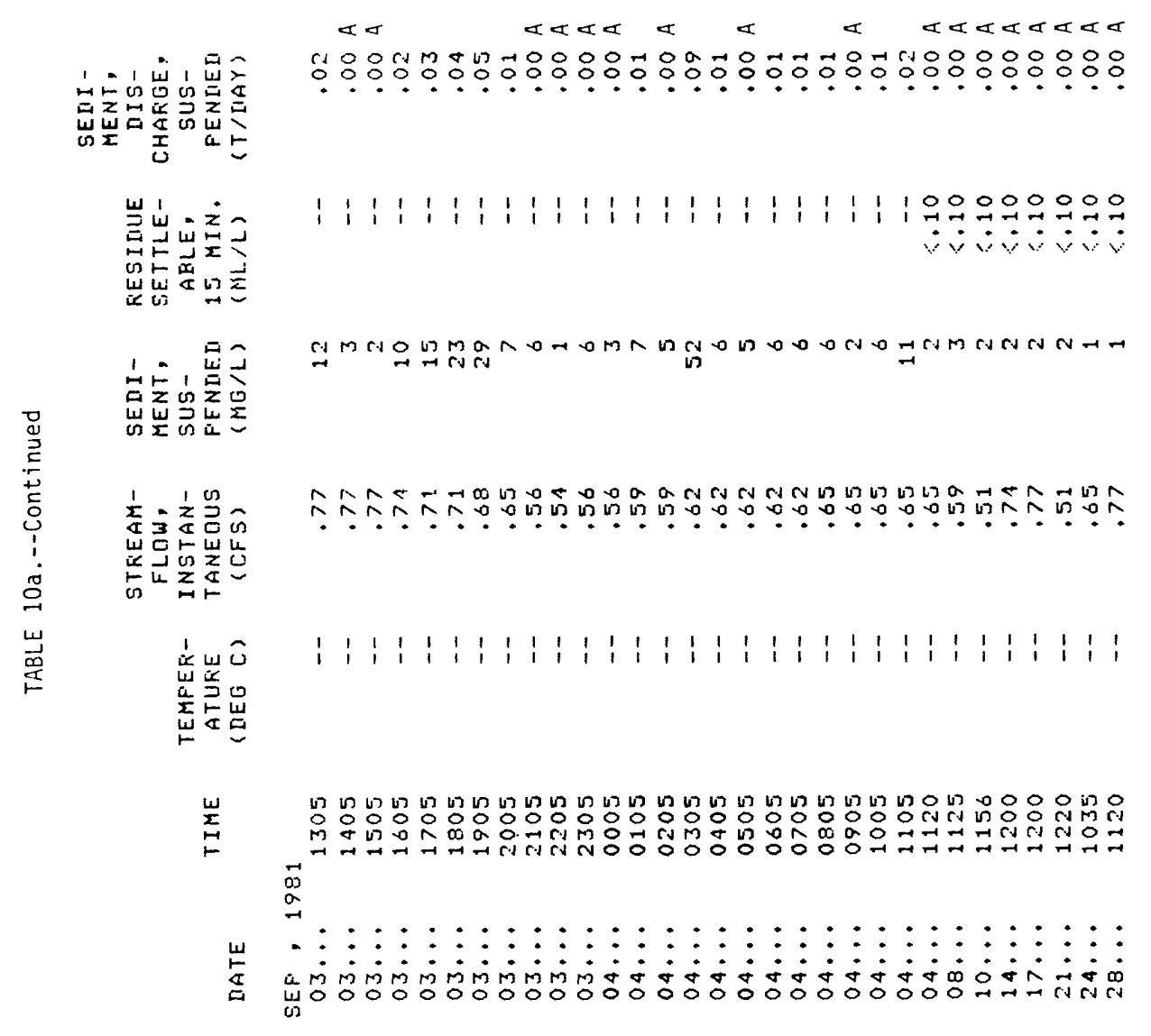




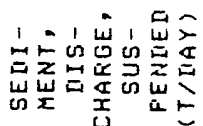

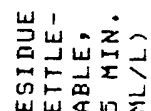

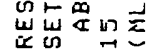

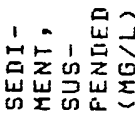

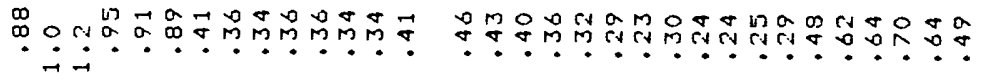

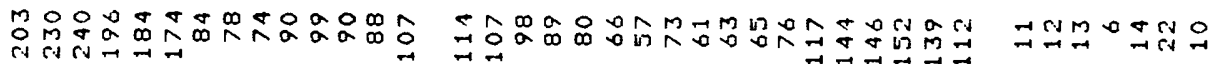

$\pm$

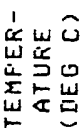
$\underset{⿱ ⺊}{a}$

\section{岂光。}

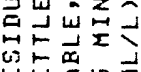

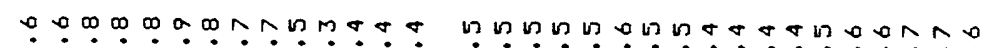

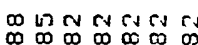

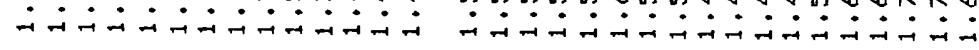

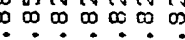
$\stackrel{a}{a}$

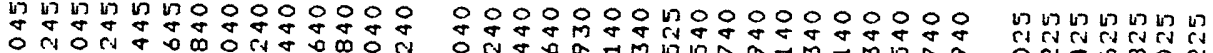
$a^{a+1}$

$\begin{array}{lllllllllllllllllllllllllllllllllllllll}1 & 1 & 1 & 1 & 1 & 1 & 1 & 1 & 1 & 1 & 1 & 1 & 1 & 1 & 1 & 1 & 1 & 1 & 1 & 1 & 1 & 1 & 1 & 1 & 1 & 1 & 1 & 1 & 1 & 1 & 1 & 1 & 1 & 1 & 1 & 1 & 1 & 1 & 1\end{array}$

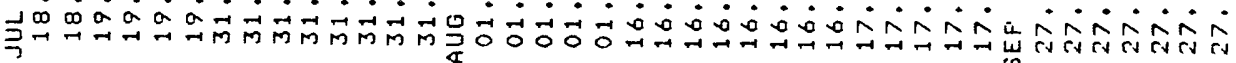

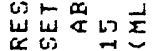

$\stackrel{m}{\sim} \underset{m}{m}$

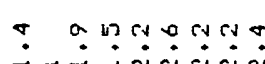

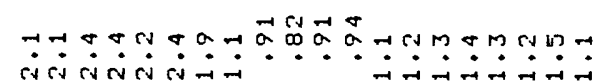

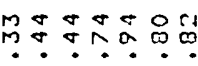

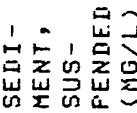

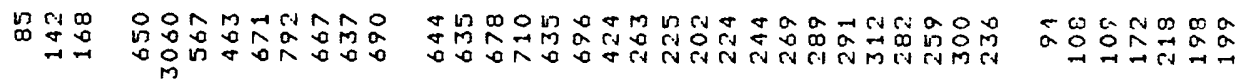

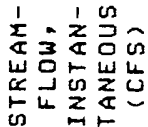

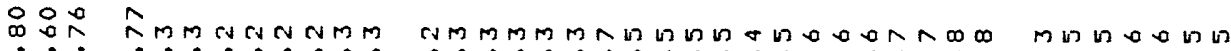
山ा

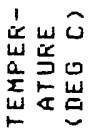

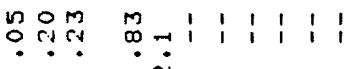

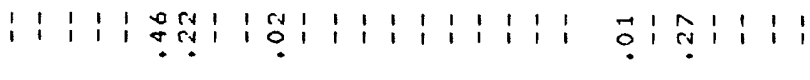

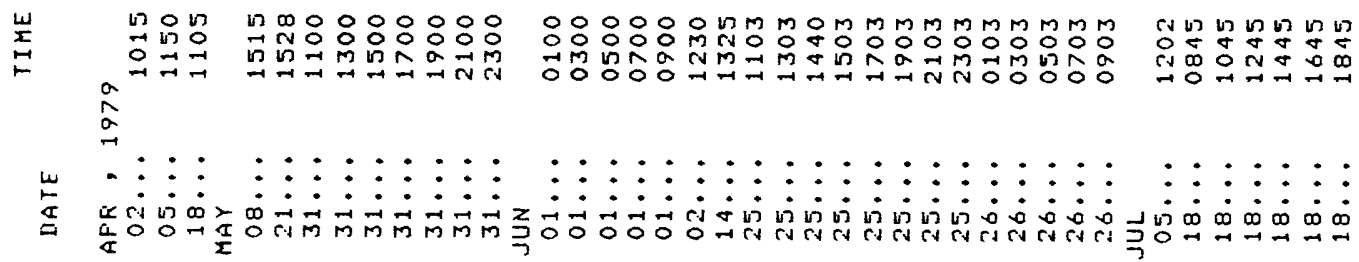




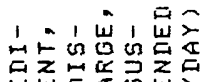

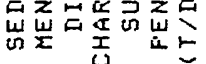

岂山豞今

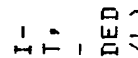
品壳记至气

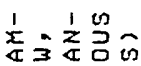

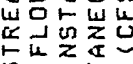

c口 40 a

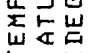
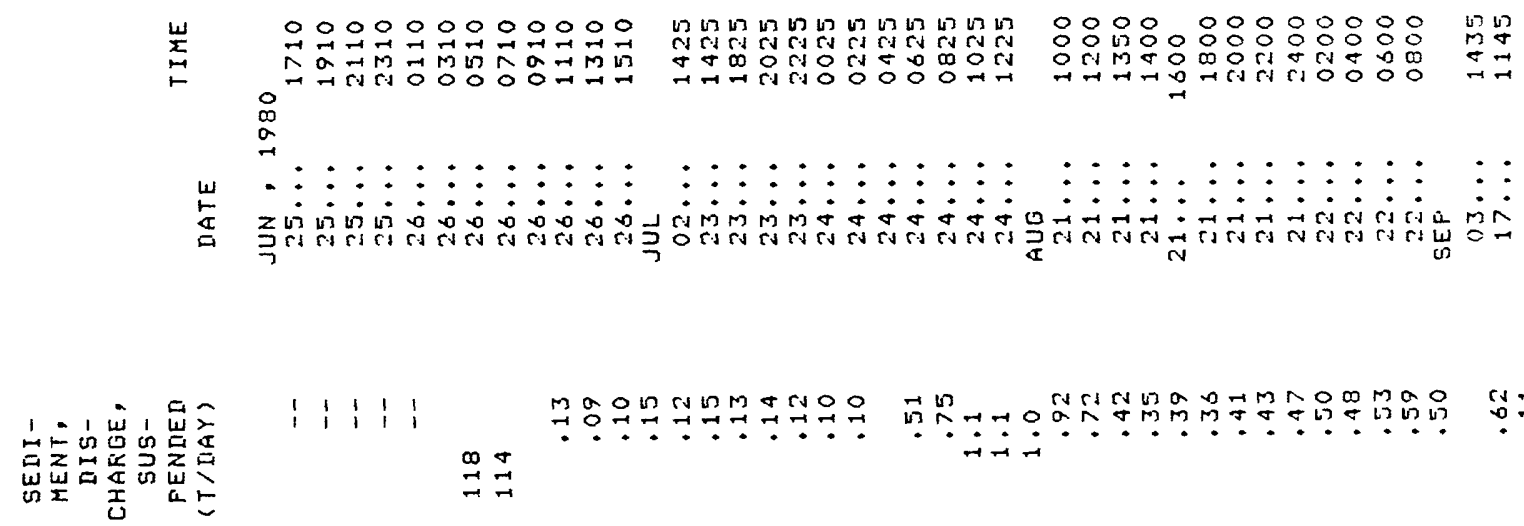

1 1111 $\stackrel{\infty}{\exists} \underset{二}{二}$

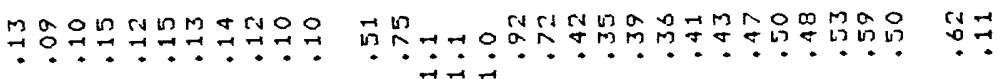

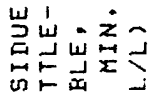

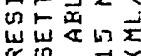

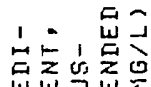

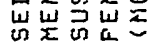

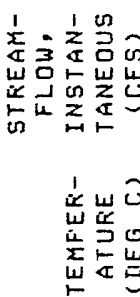

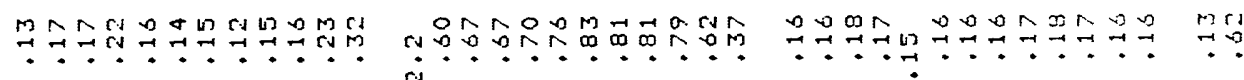

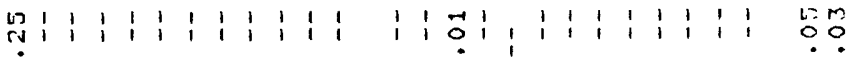

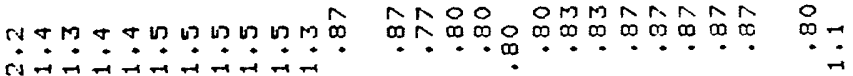

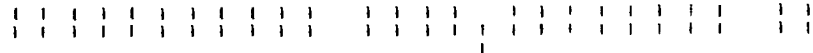

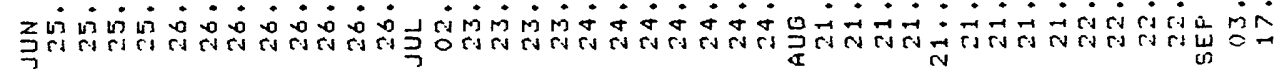

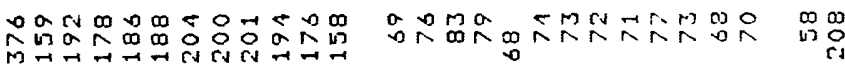

$1: 1$ $\underset{m}{\ddot{n}} \boldsymbol{0}$

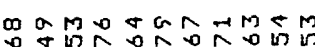

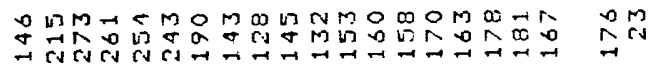

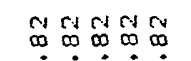
om

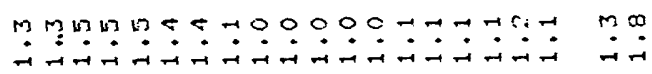

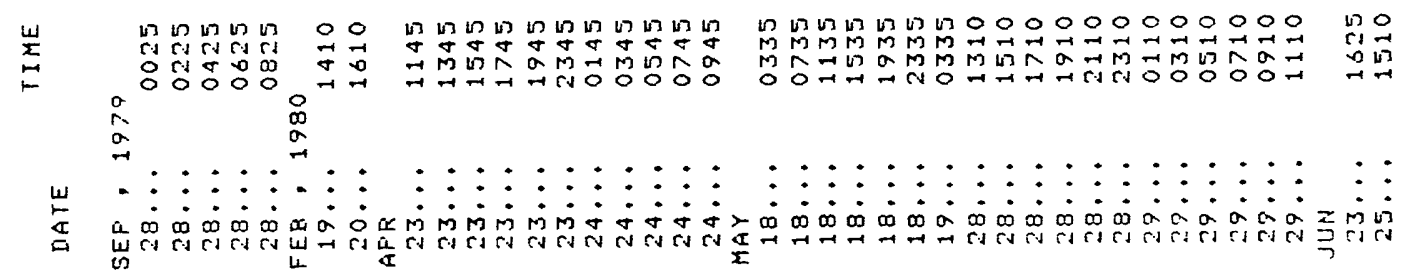




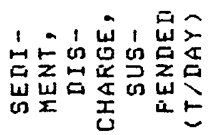

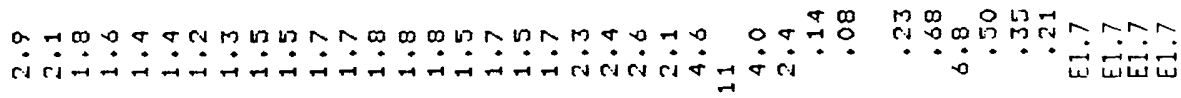

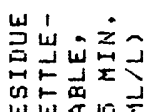

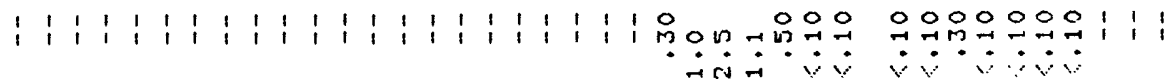

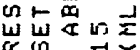

它它的范主

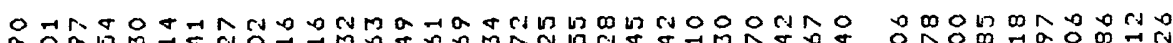

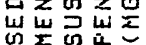

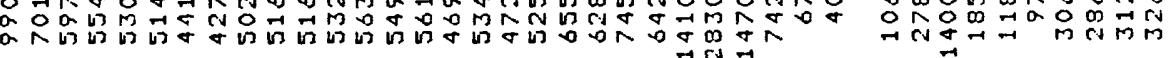

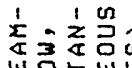

崖过占岩岂

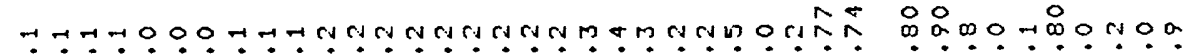

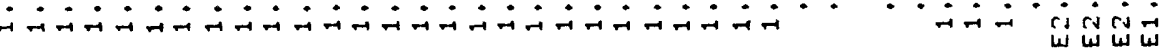

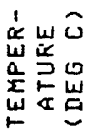
$\begin{array}{llllllllllllllllllllllllllllllllllllll}1 & 1 & 1 & 1 & 1 & 1 & 1 & 1 & 1 & 1 & 1 & 1 & 1 & 1 & 1 & 1 & 1 & 1 & 1 & 1 & 1 & 1 & 1 & 0 & 1 & 1 & 1 & 1 & 1 & 1 & 1 & 1 & 1 & 1 & 1 & 1 & 1\end{array}$

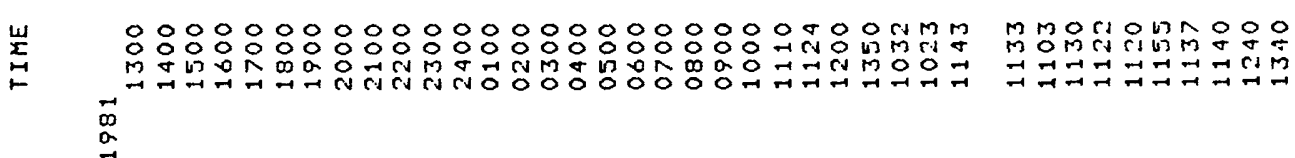

岂出出突

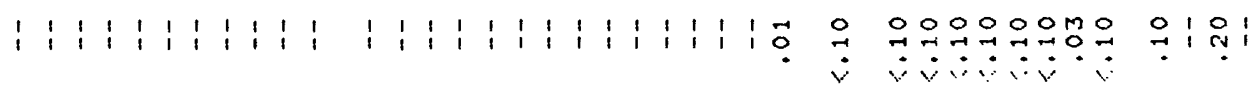

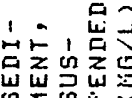

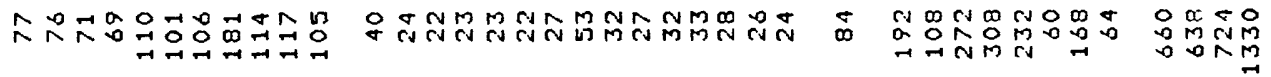

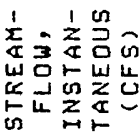
$\because 000$

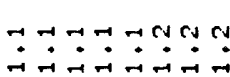

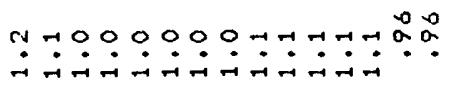<smiles>c1ccccc1</smiles>

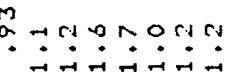
운?

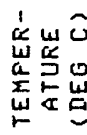

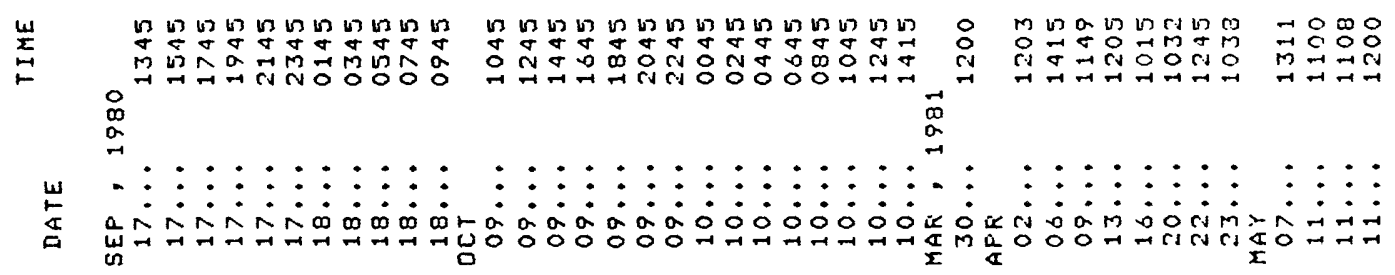




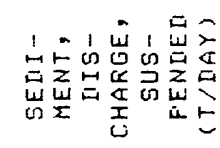

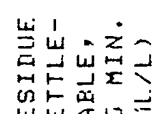

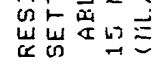

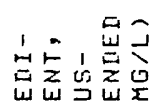

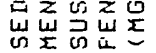

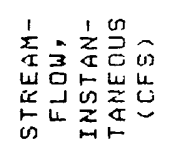

îे

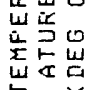

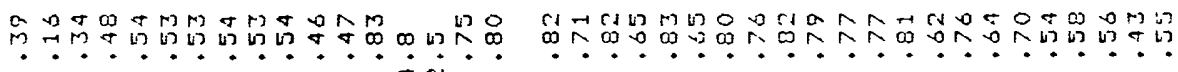
$\dot{\alpha}{ }^{\circ}$

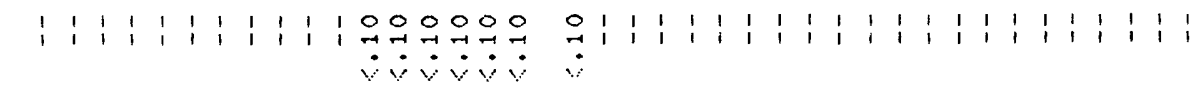

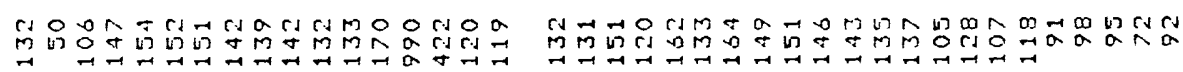

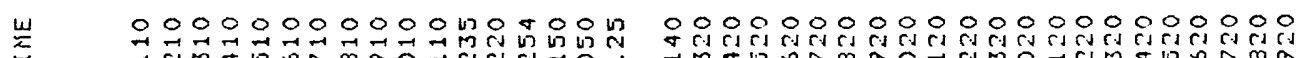

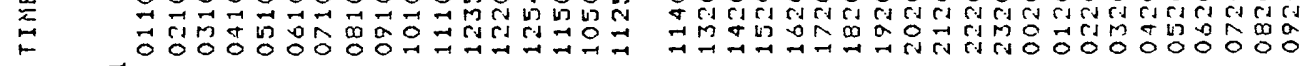

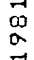

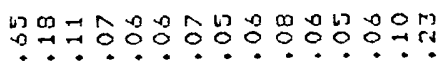

岂崖出的

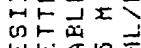

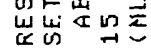

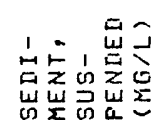
$\begin{array}{llllllllllllllllllllllllllllllllllllllll}1 & 1 & 1 & 1 & 1 & 1 & 1 & 1 & 1 & 1 & 1 & 1 & 1 & 1 & 1 & 1 & 1 & 1 & 1 & 1 & 1 & 1 & 0 & 0 & 0 & 0 & 1 & 1 & 1 & 1 & 1 & 1 & 1 & 1 & 1 & 1 & 1 & 1 & 1 & 1\end{array}$ $\sum_{x}^{1} \sum_{x}^{1} \frac{0}{\partial}$

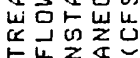

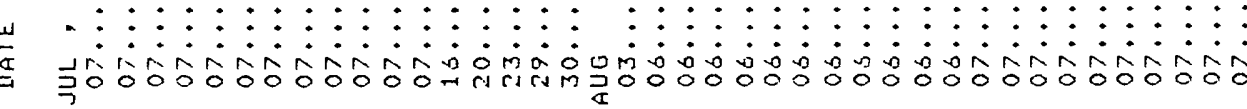
の 它岕它

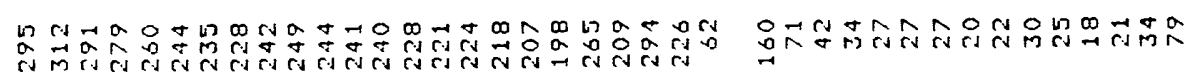

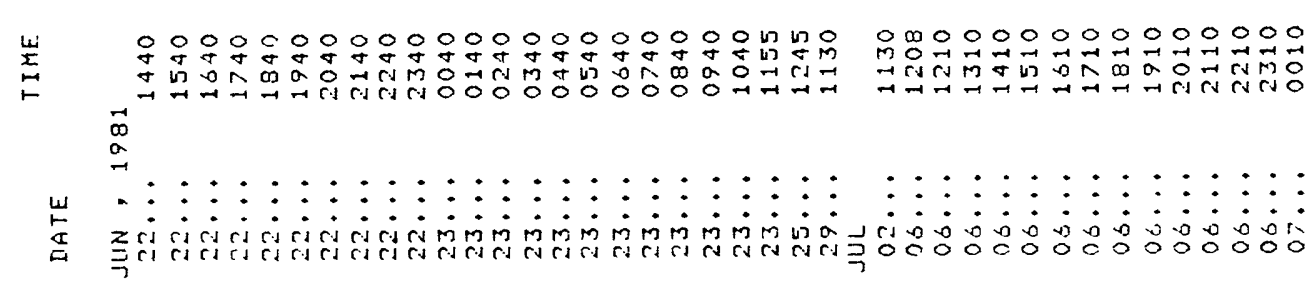

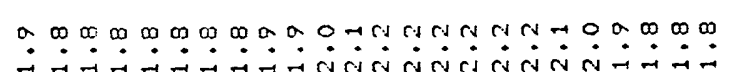

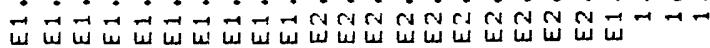

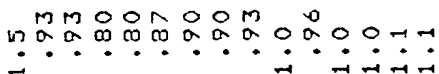
$\begin{array}{lllllllllllllllllllllllllllllllllllllll}1 & 1 & 1 & 1 & 1 & 1 & 1 & 1 & 1 & 1 & 1 & 1 & 1 & 1 & 1 & 1 & 1 & 1 & 1 & 1 & 0 & 1 & 1 & 1 & 1 & 1 & 1 & 1 & 1 & 1 & 1 & 1 & 1 & 1 & 1 & 1 & 1 & 1\end{array}$ 


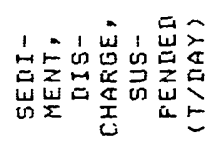

ging

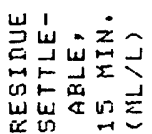

엉ㅇㅇㅇㅇ

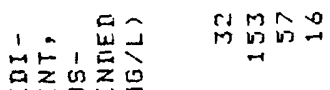

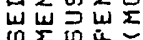

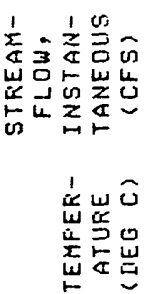

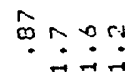

ar

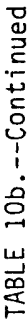

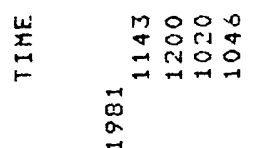

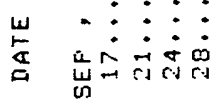

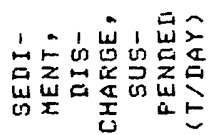

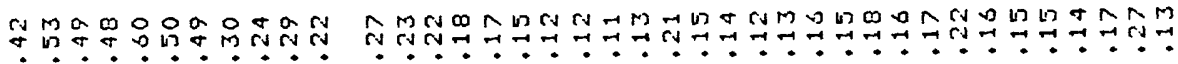

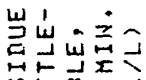

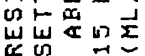

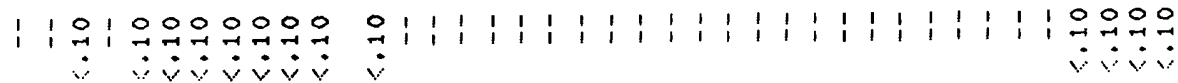

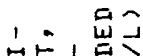

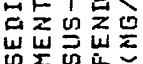

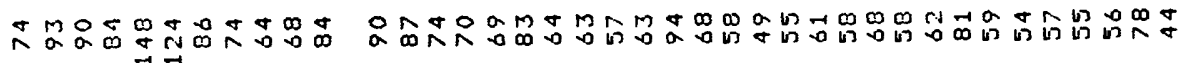

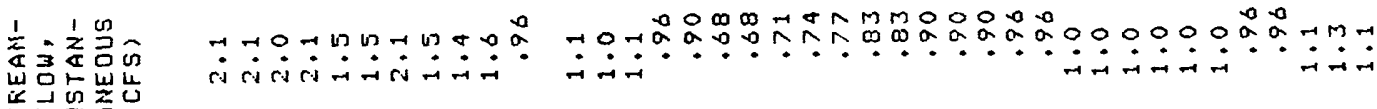

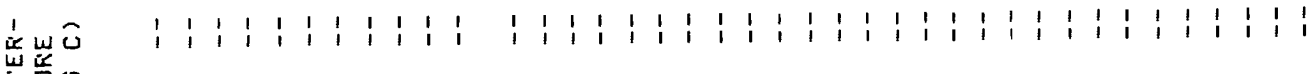

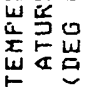

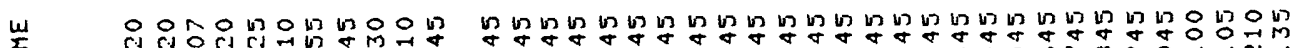

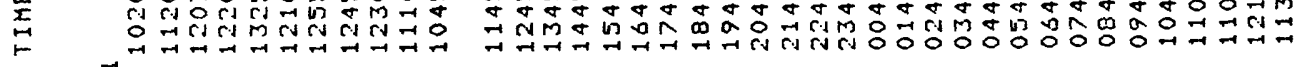
$\overrightarrow{0}$

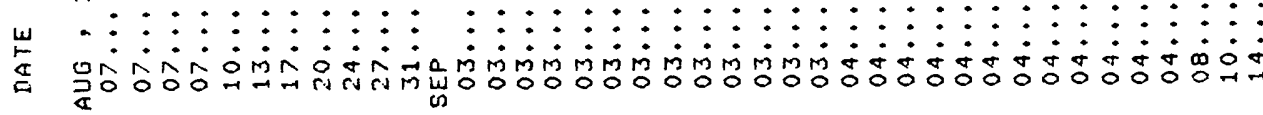




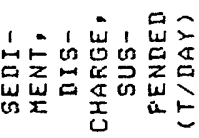

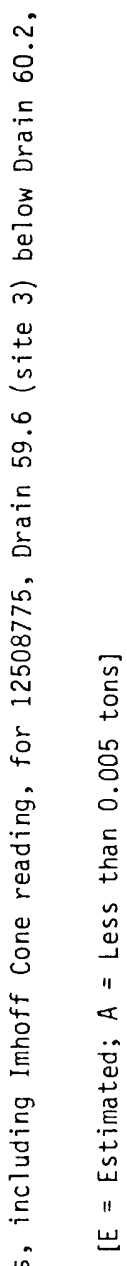

岂出安

台云的总导

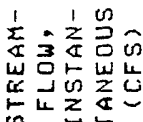

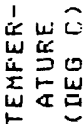

$\underset{⿱ 亠 䒑}{\stackrel{w}{a}}$

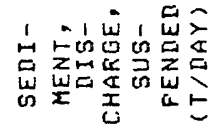

$\omega 1, \dot{m}$

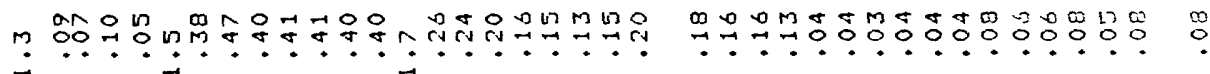

$\begin{array}{llllllllllllllllllllllllllllllllllllll}n & 0 & 1 & 0 & 1 & 1 & 1 & 1 & 1 & 1 & 1 & 1 & 1 & 1 & 1 & 1 & 1 & 1 & 1 & 1 & 1 & 1 & 1 & 1 & 1 & 1 & 1 & 1 & 1 & 1 & 1 & 1 & 1 & 1 & 1 & 1 & 1 & 1 \\ 0\end{array}$

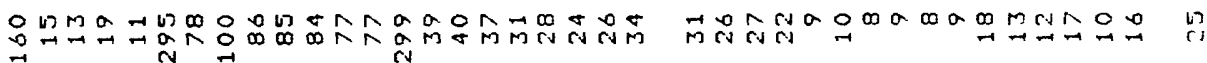

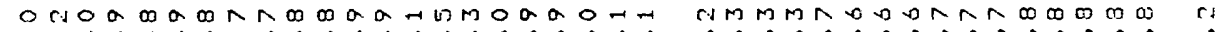

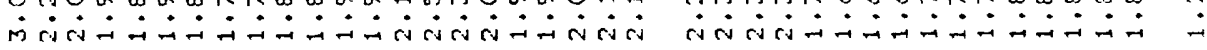

$\begin{array}{lllllllllllllllllllllllllllllllllllllll}1 & 1 & 1 & 1 & 1 & 1 & 1 & 1 & 1 & 1 & 1 & 1 & 1 & 1 & 1 & 1 & 1 & 1 & 1 & 1 & 1 & 1 & 1 & 1 & 1 & 1 & 1 & 1 & 1 & 1 & 1 & 1 & 1 & 1 & 1 & 1 & 1 & 1 & 1\end{array}$

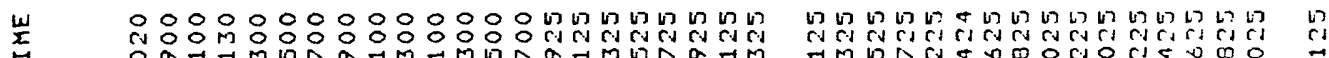
$a^{a}$

영 $\exists=\pi$ m

$:::::::::::::::::::::$

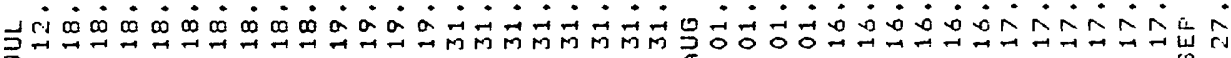

オ

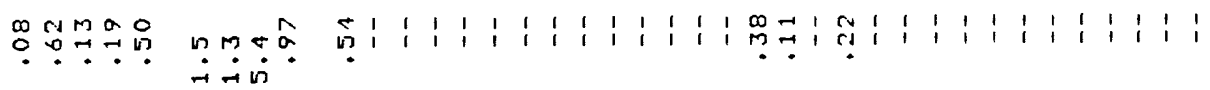

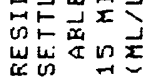

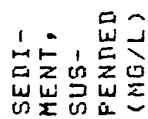

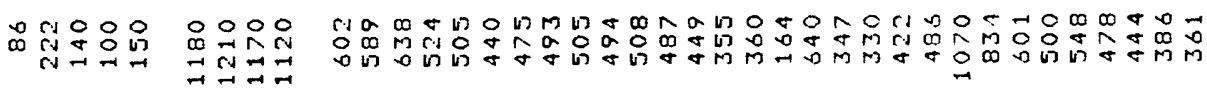

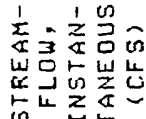

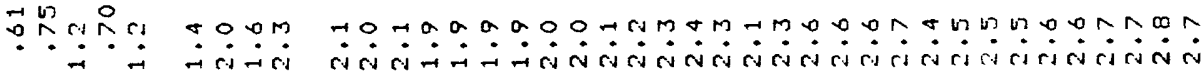

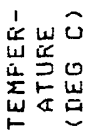

\begin{tabular}{lllllllllllllllllllllllllll}
1 & 1 & 1 & 1 & 1 & 1 & 1 & 1 & 1 & 1 & 1 & 0 & 1 & 1 & 1 & 1 & 1 & 1 & 1 & 1 & 1 & 1 & 1 & 1 & 1 & 1 & 1 \\
\hline
\end{tabular}

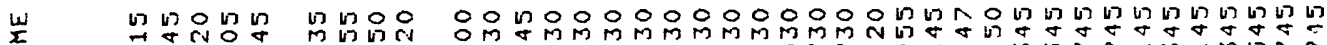

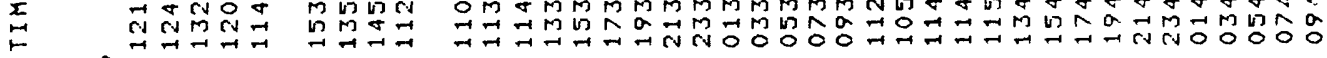
$a$

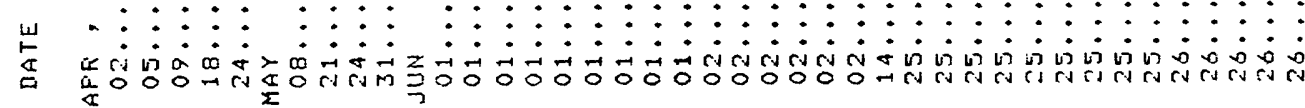




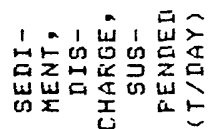

岂岕战忐引

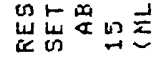

$1 . \quad 5$

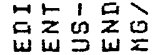

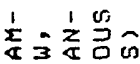

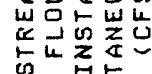

岳岂

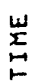

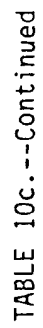

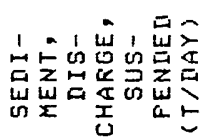

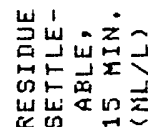

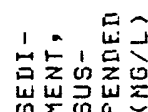

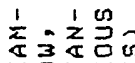

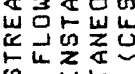

岳崖它

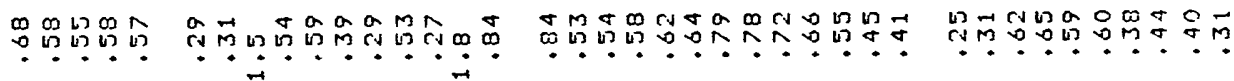

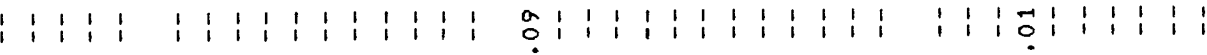

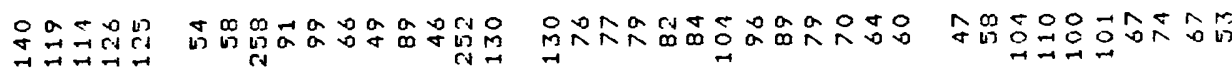

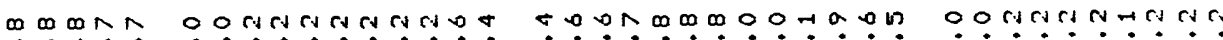

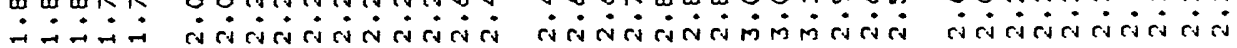
(2)

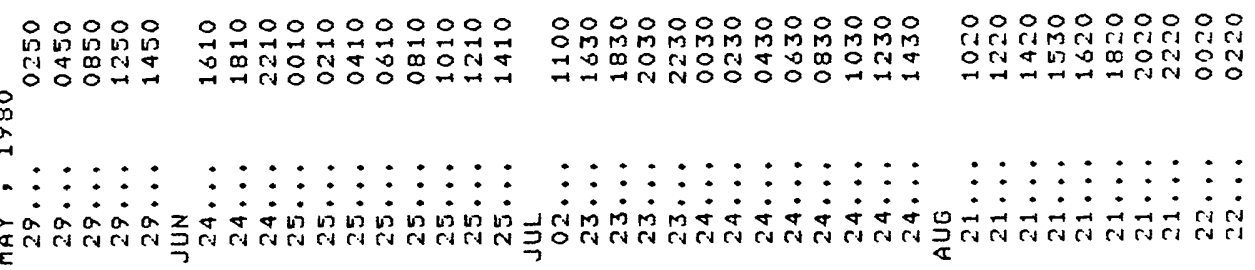

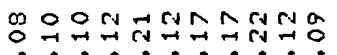

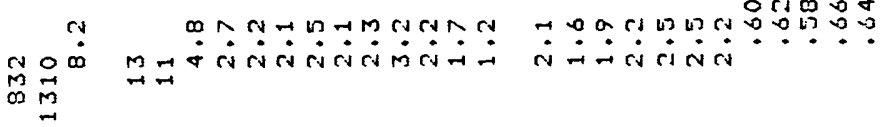

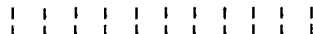

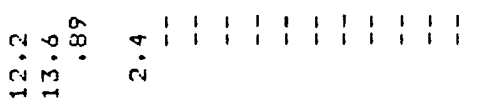

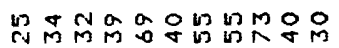

$\circ \therefore$
$\therefore$
0

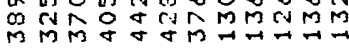

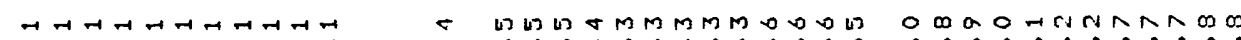

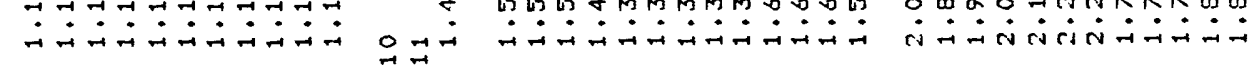

\section{$\stackrel{-1}{i}$}

$\begin{array}{lllllllllllll}1 & 1 & 1 & 1 & 1 & 1 & 1 & 1 & 1 & 1 & 1 & 1 \\ 0\end{array}$

$\begin{array}{lllllllllll}1 & 1 & 1 & 1 & 1 & 1 & 1 & 1 & 1 & 1 & 1 \\ 1 & & & & & & & \end{array}$

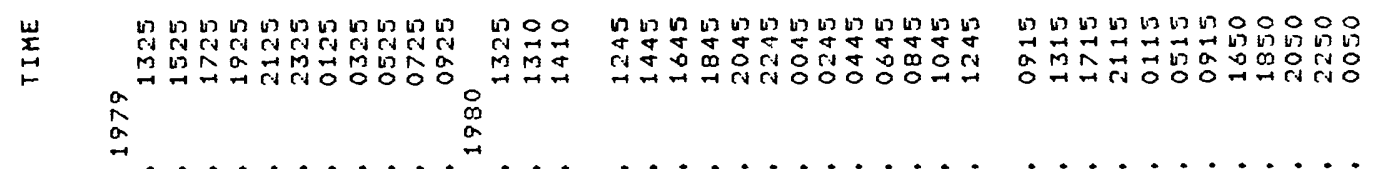

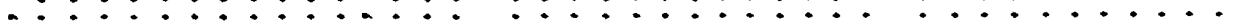
a

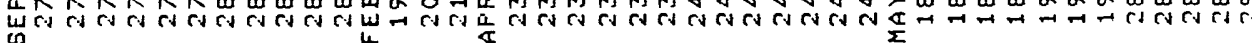




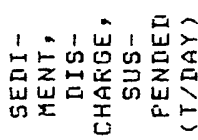

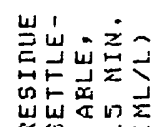

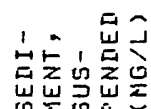

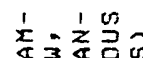

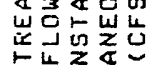

\&u

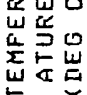

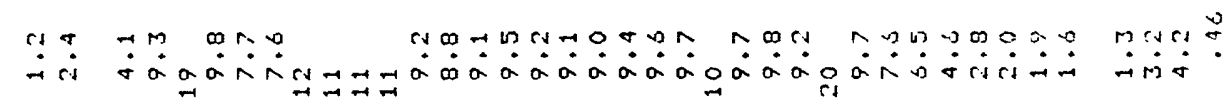

암

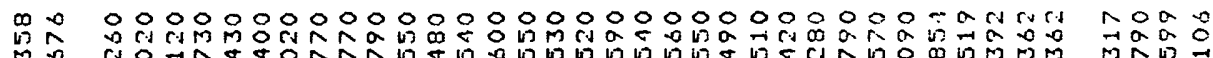

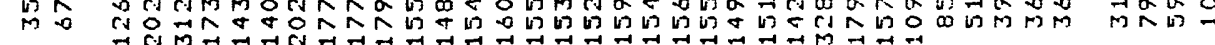

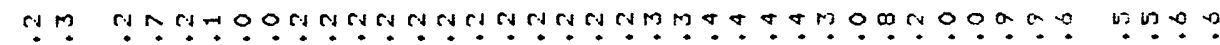

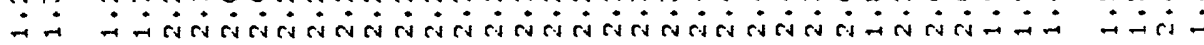

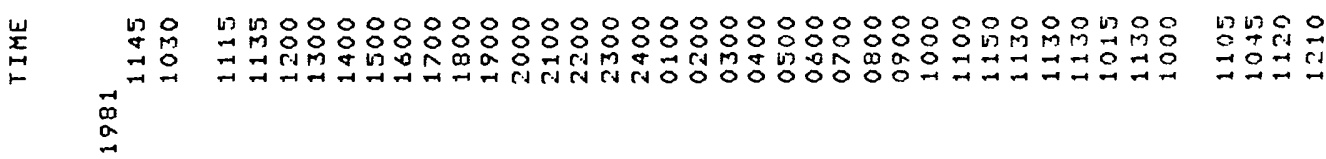

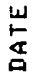

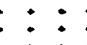

$\ddot{m} \dot{2} \dot{0} \dot{0}$

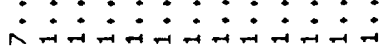

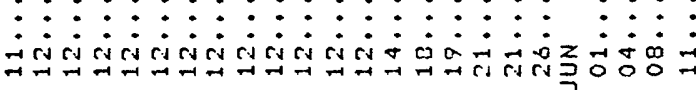

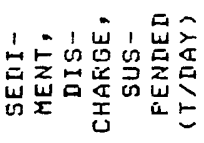

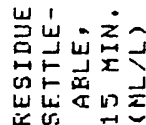

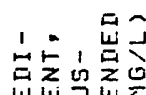

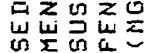

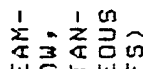

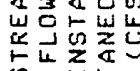

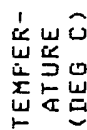

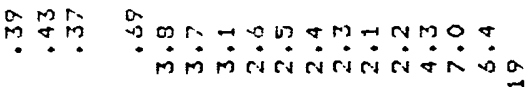

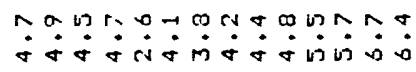

$i$
$i$ \begin{tabular}{c:c|c}
$m$ & -1 \\
0 & 1 & 0 \\
0
\end{tabular} no 0

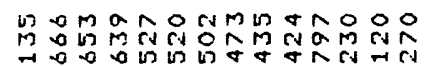
$\lim _{n \rightarrow \infty}$

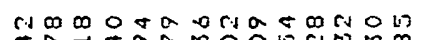

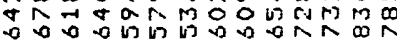

$\therefore \quad \alpha 0000 \cos$

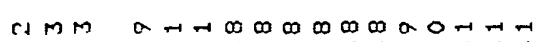

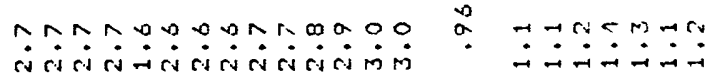
arci

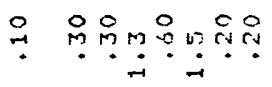

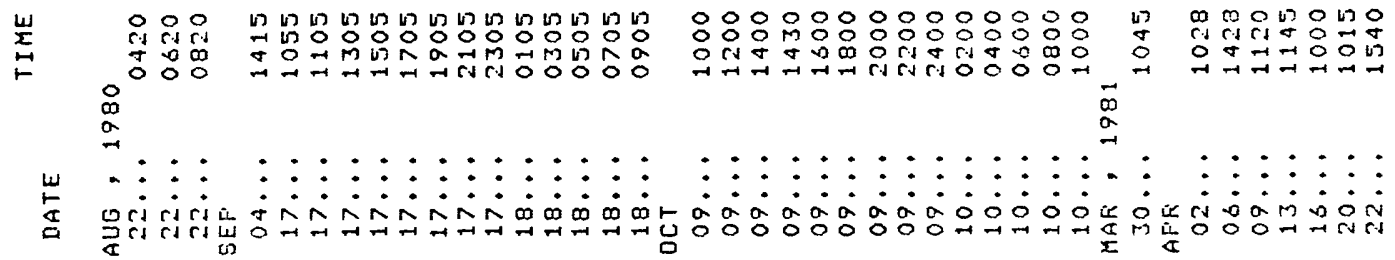




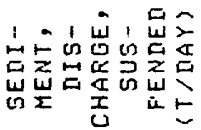

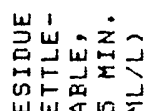

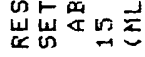

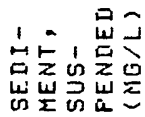

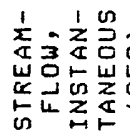

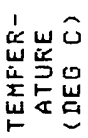

n-n n

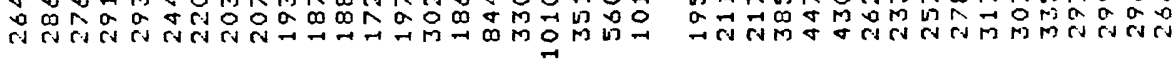

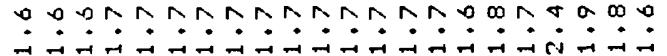

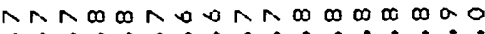

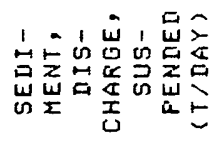

岂息的主

官占的崖号

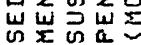

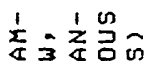

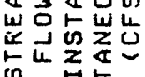

$\ll \tilde{\omega}$ 造崖

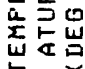

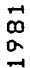

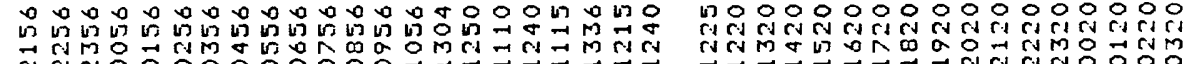

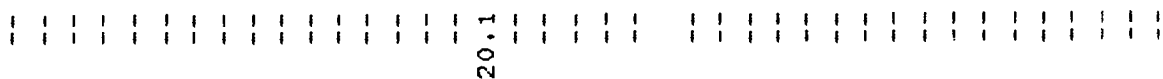

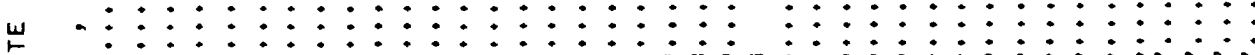

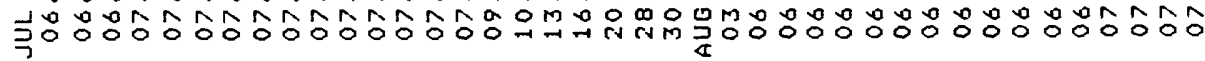

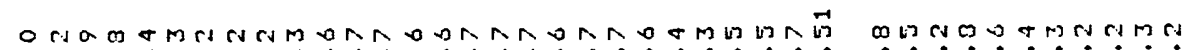

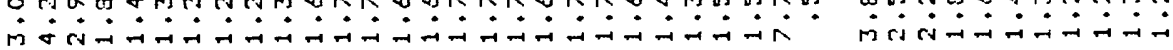

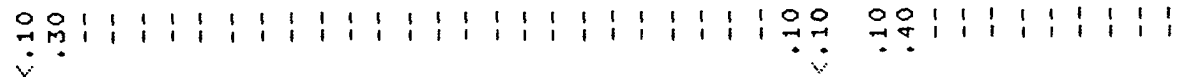

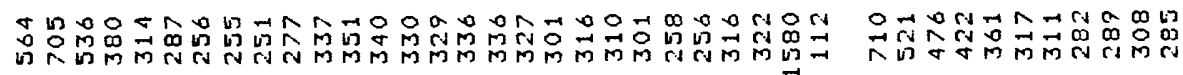

ONODNMNNRN

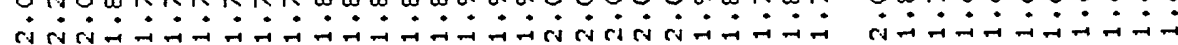

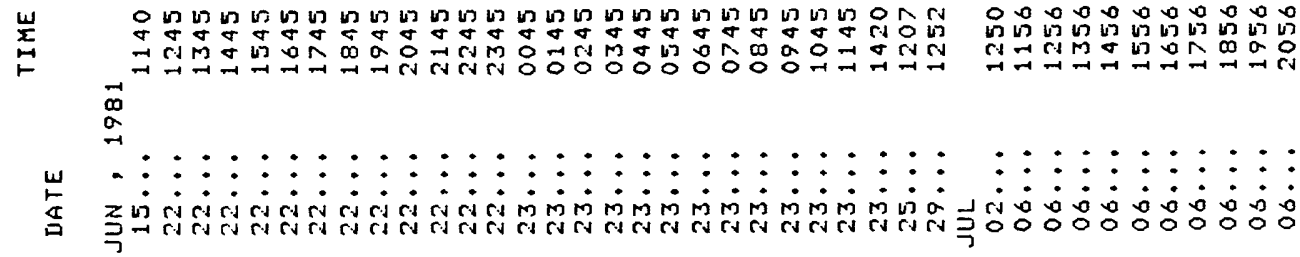




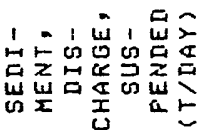

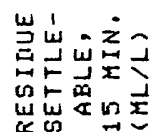

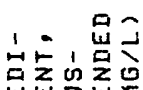

岁崖色岩要

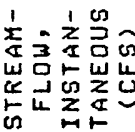

\& $\omega \hat{0}$

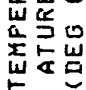

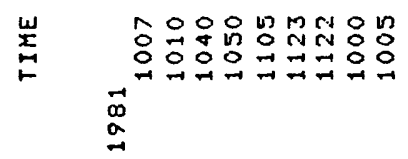

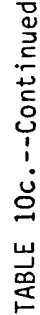

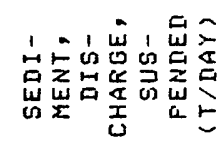

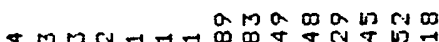

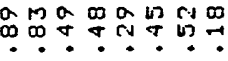

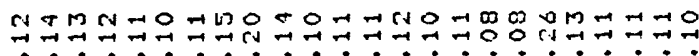

岂出的齐

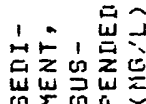

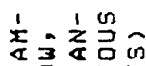

w西高皆

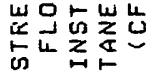

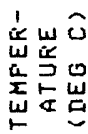

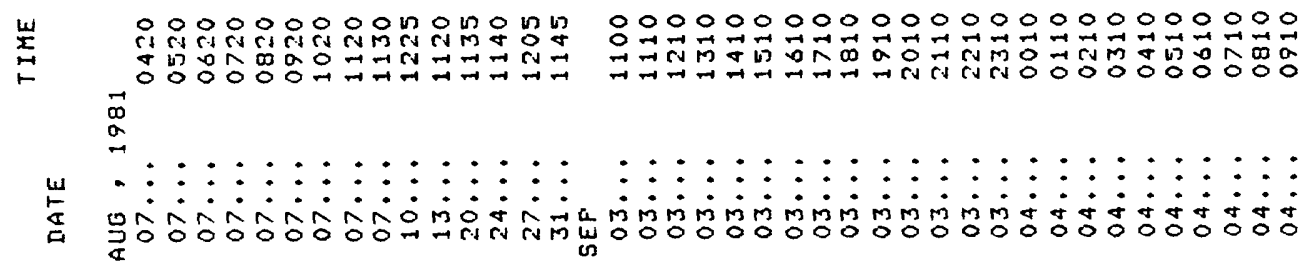




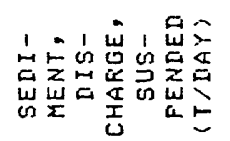

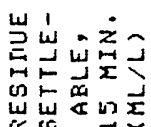

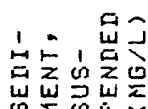

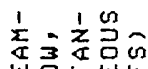

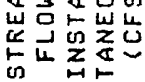

离岕

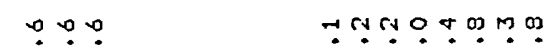

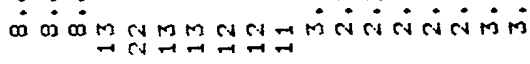

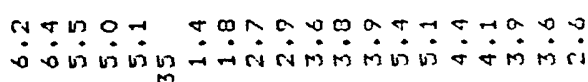

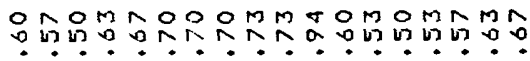

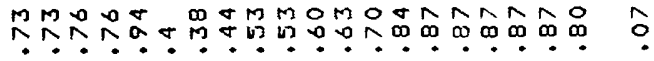

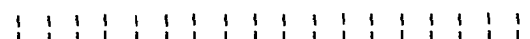

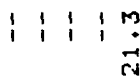

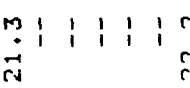

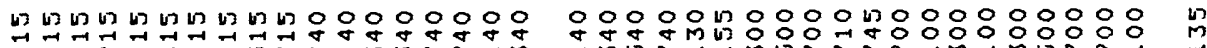
$\stackrel{a}{a}$

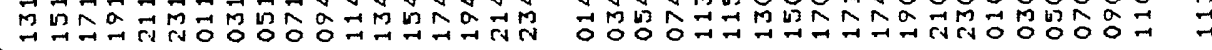

$:::::::::::::::::::$

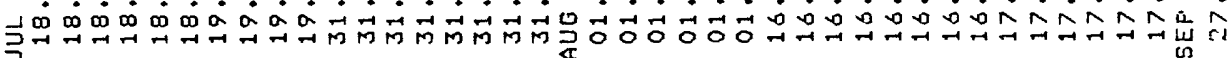

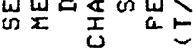

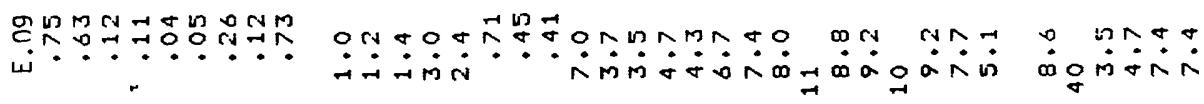

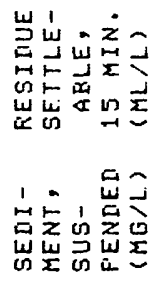

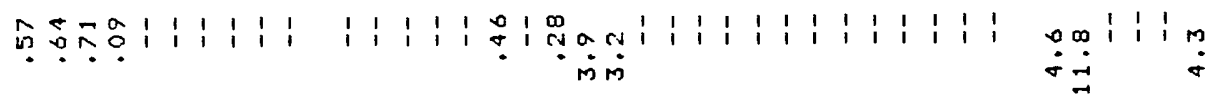

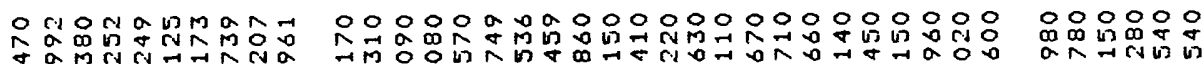

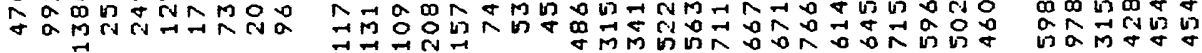

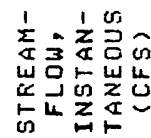

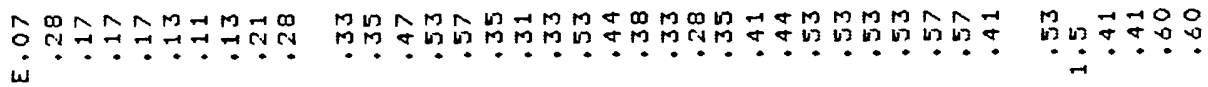

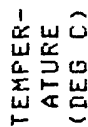

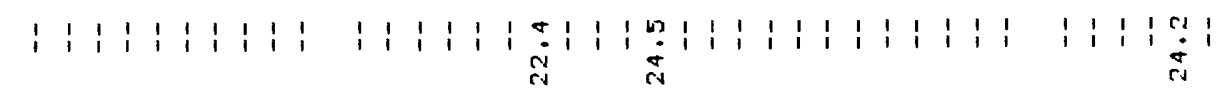

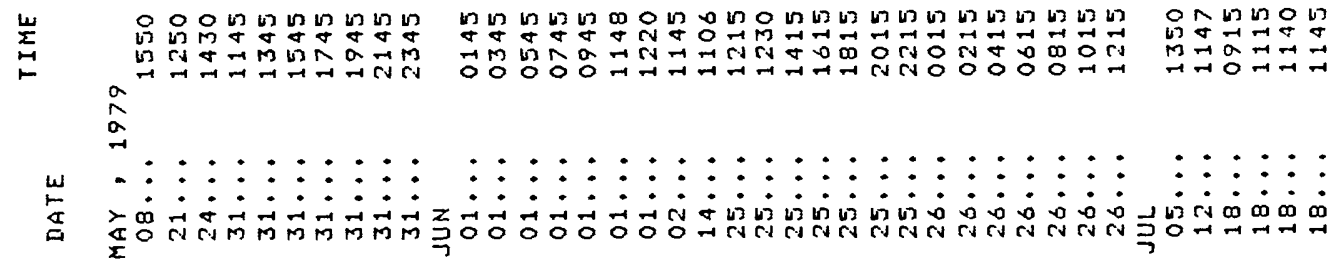




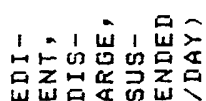

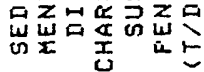

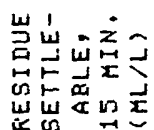

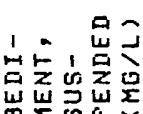

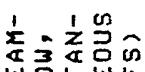

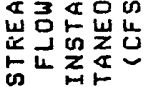

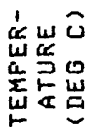

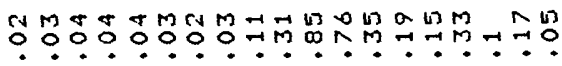

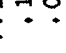

4m $\dot{8} \dot{8}$

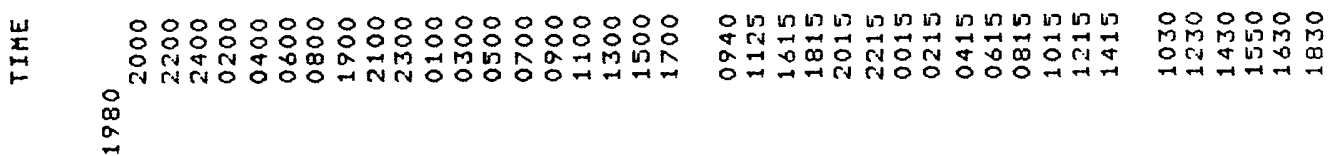

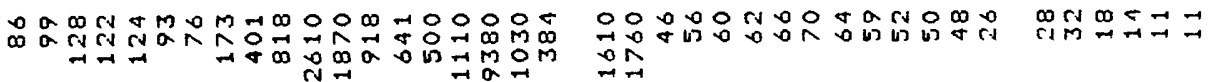

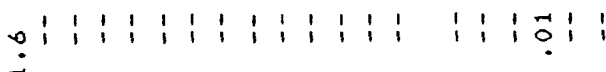

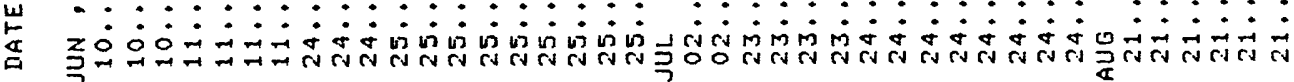

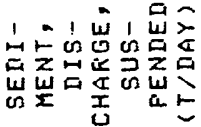

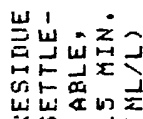
岳的崖导

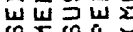

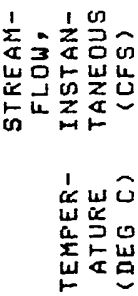

$<<<<<<<<<<<$

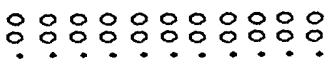

$\pi$
4
0

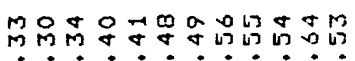

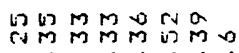

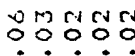

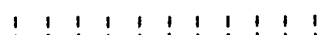

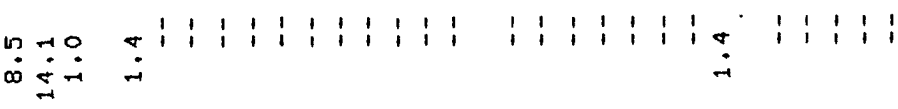
nan+0000m+o 응요

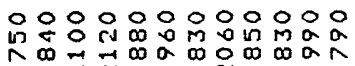

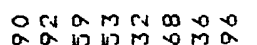

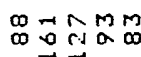

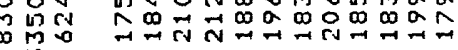

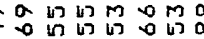

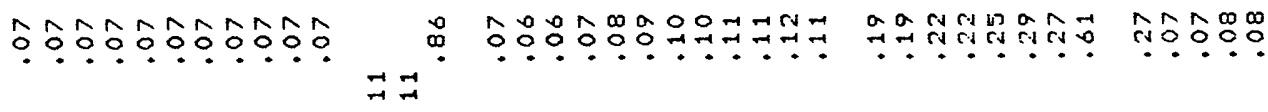

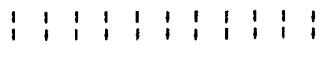

$$
\begin{aligned}
& \text { is } \\
& 11119191191 \\
& \begin{array}{lllllll}
1 & 1 & 1 & 1 & 1 & 1 & 0 \\
2 & 0
\end{array}
\end{aligned}
$$

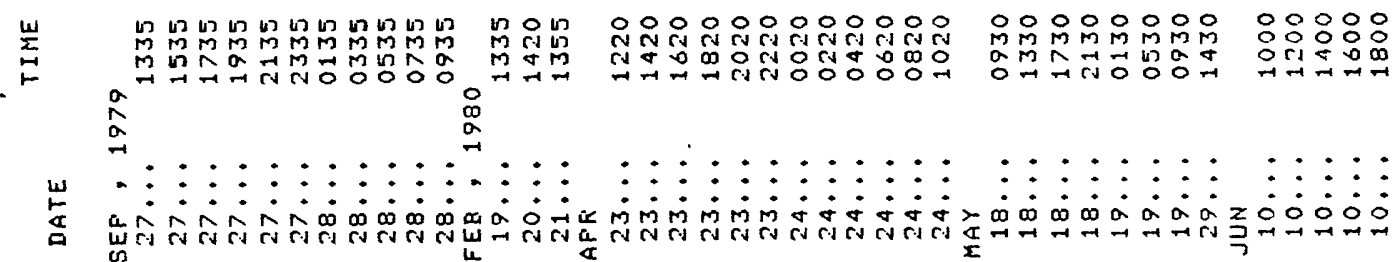




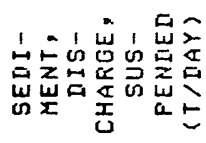

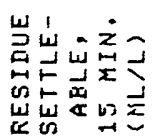

的它的究引

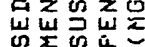

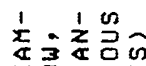

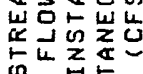
岳崖它

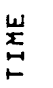

恖

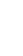

: : : : :

$11 \stackrel{0}{1} 111$

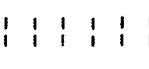

$\therefore \circ 0 \circ \div 0$

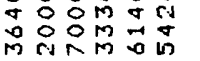

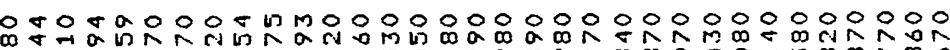

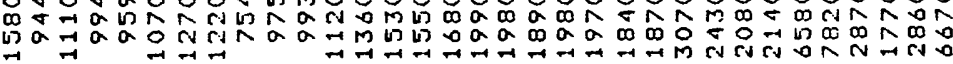

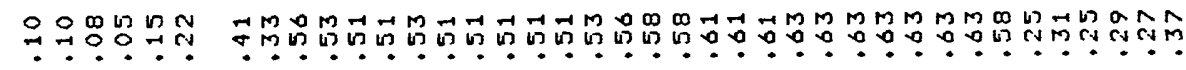

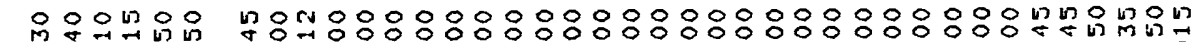

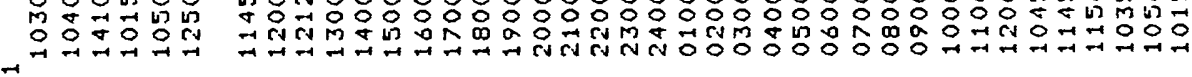
a

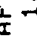

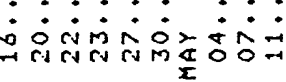
更

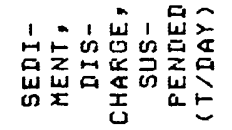

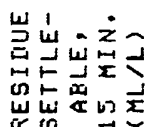

1을

店它

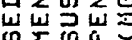

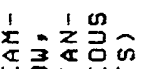

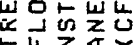

的始远

$\dot{1} \omega \hat{0}$

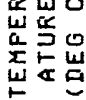

$14<<<\ll<4$
0000000

$:: 8:: 8:$ $\therefore$

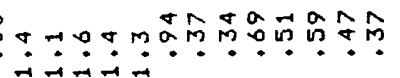

$<\ll<<<<<<<<<$

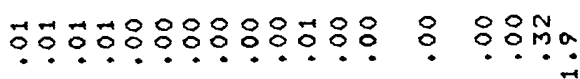

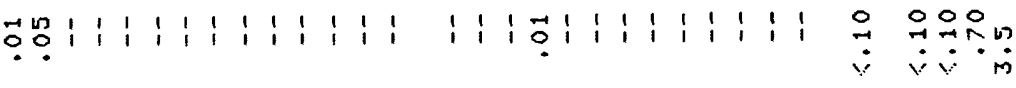

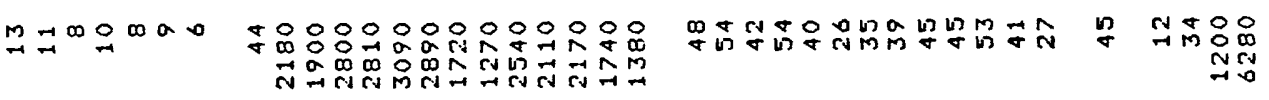

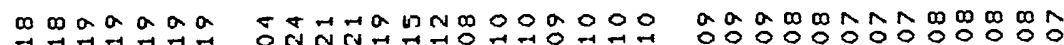
$\stackrel{+}{0}: \stackrel{0}{0} \div$

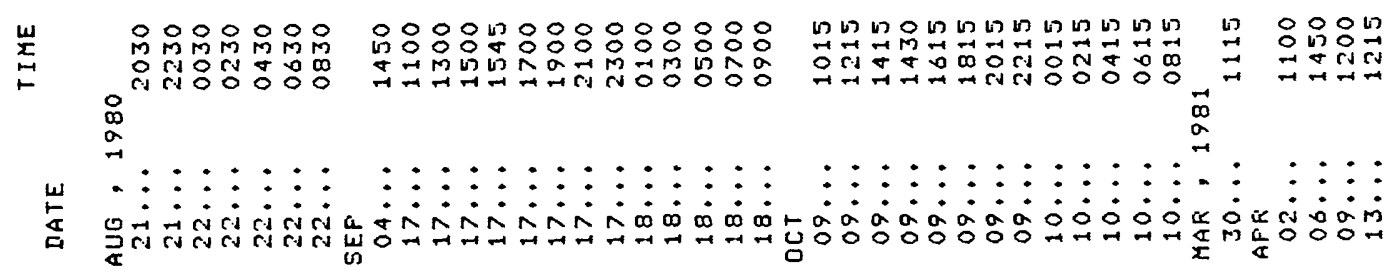




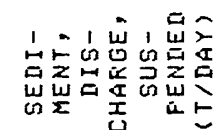

岂岕的竧

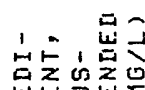

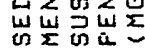

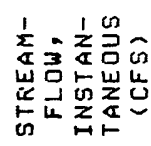

10

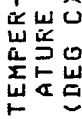

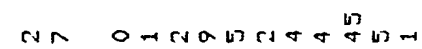

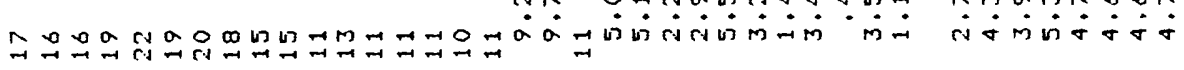

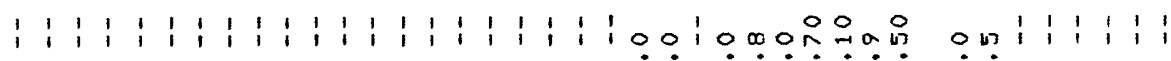

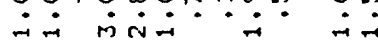

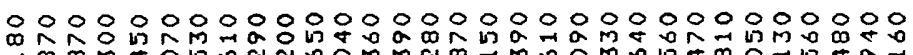

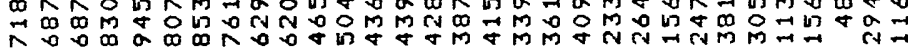

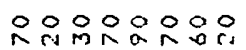

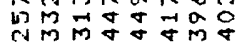

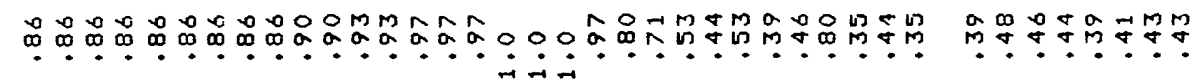

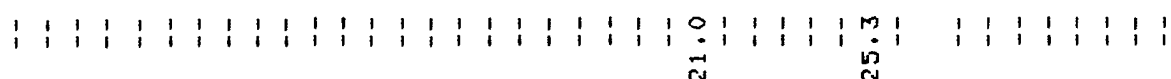

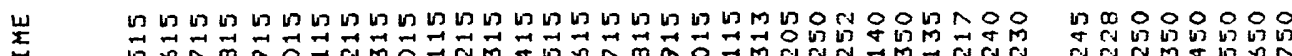

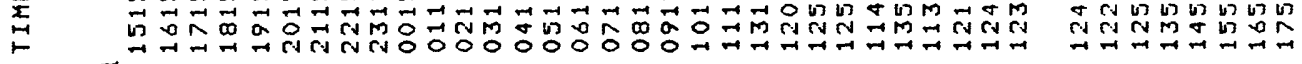
$\vec{\infty}$

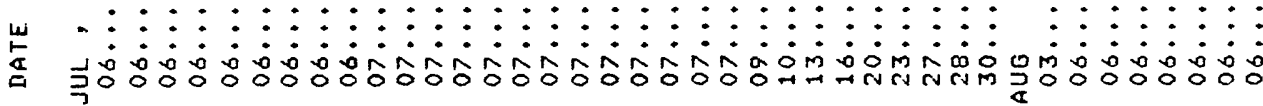

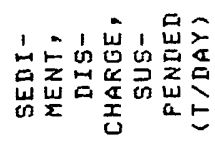

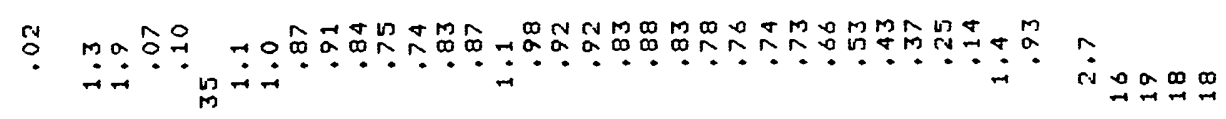

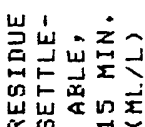

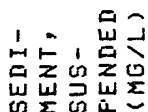

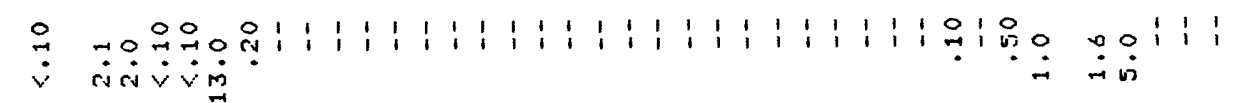

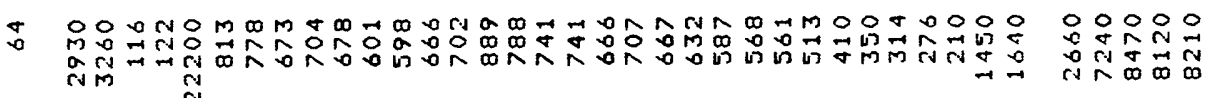
$\sum_{x}=\sum_{x}^{1} \frac{v_{i}}{a} \hat{a}$

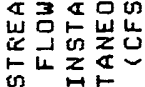

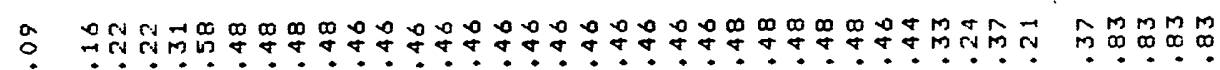

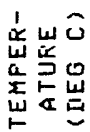
$\begin{array}{lllllllllllllllllllllllllllllllllllll}1 & 1 & 1 & 1 & 1 & 1 & 1 & 1 & 1 & 1 & 1 & 1 & 1 & 1 & 1 & 1 & 1 & 1 & 1 & 1 & 1 & 1 & 1 & 1 & 1 & 1 & 1 & 1 & 1 & 0 & 1 & 1 & 1 & 1 & 1 & 1 & 1\end{array}$

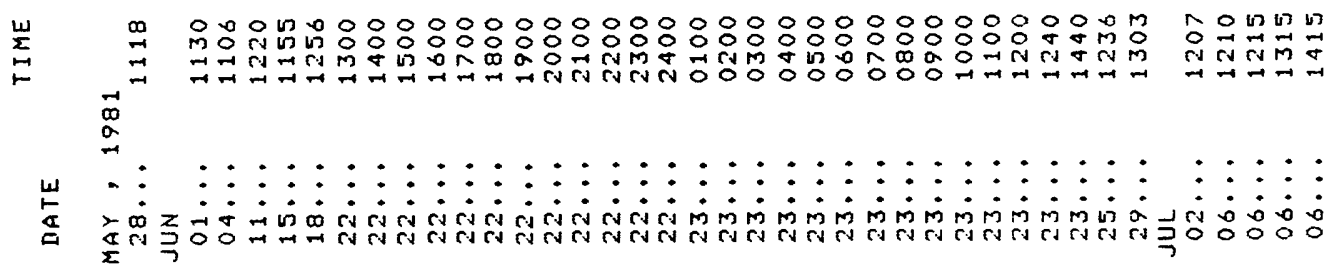




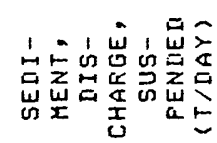

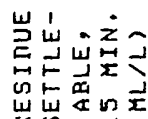

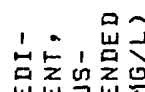

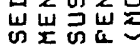

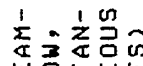
崖古出岩

的能远

岳岕岕

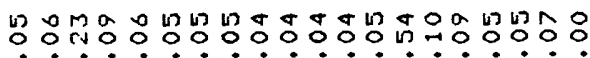

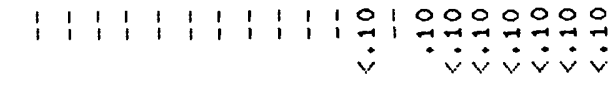

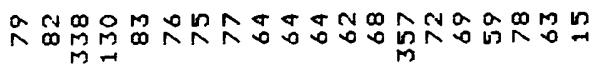

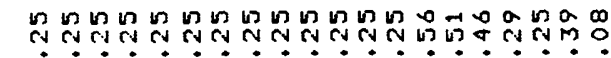

$\begin{array}{llllllllllllllllllll}1 & 1 & 1 & 1 & 1 & 1 & 1 & 1 & 1 & 1 & 1 & 1 & 1 & 1 & 1 & 1 & 1 & 1 & 1 & 1\end{array}$

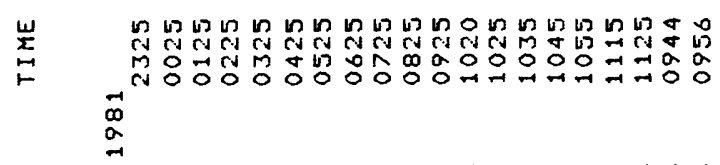

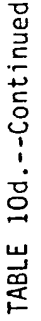

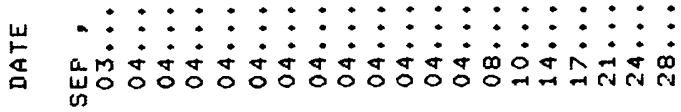

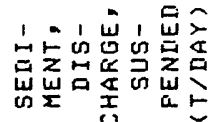

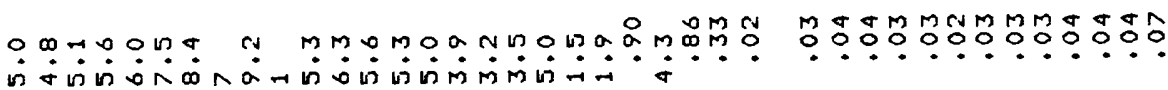

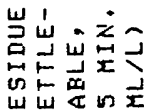

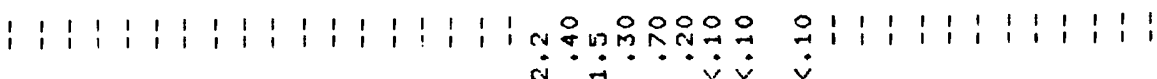

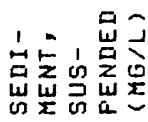

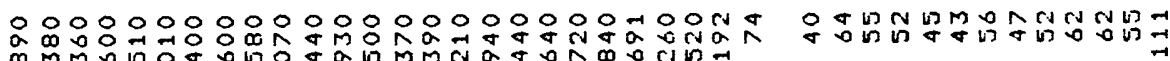

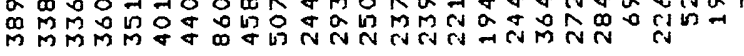

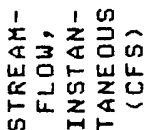

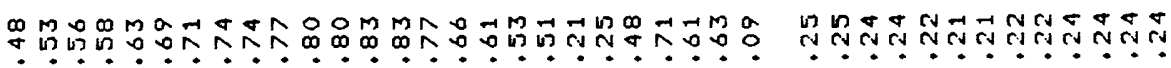

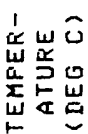

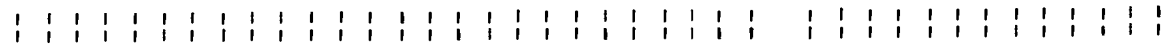

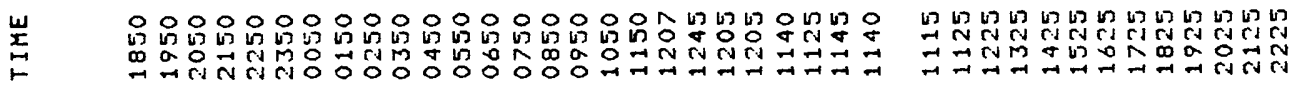
-1
0
$a$
-1

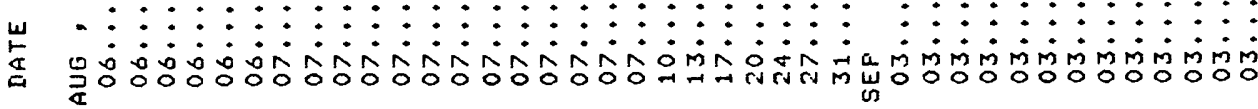


岁

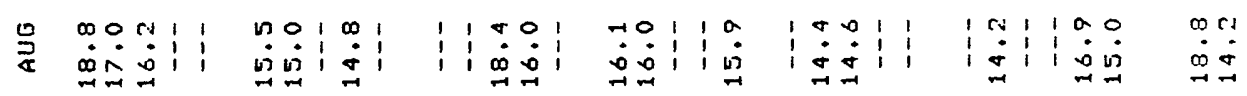

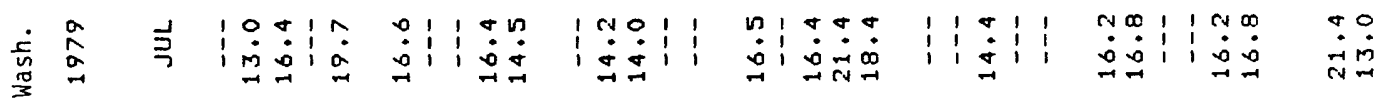
总兽

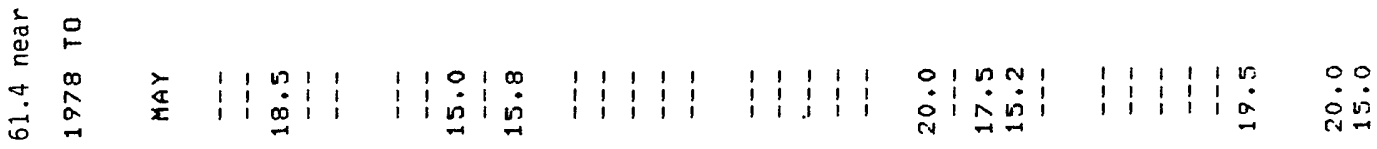

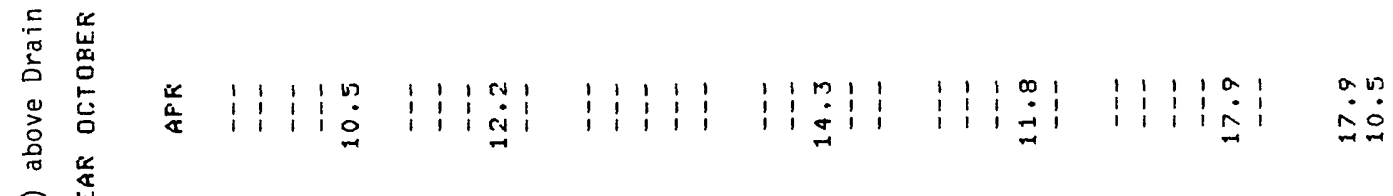

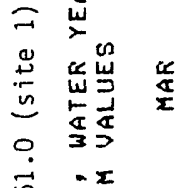

की $: \frac{x}{4}$

员

巽

兽

के

至

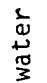

3

与

岕

을

$\stackrel{0}{=}$

崖

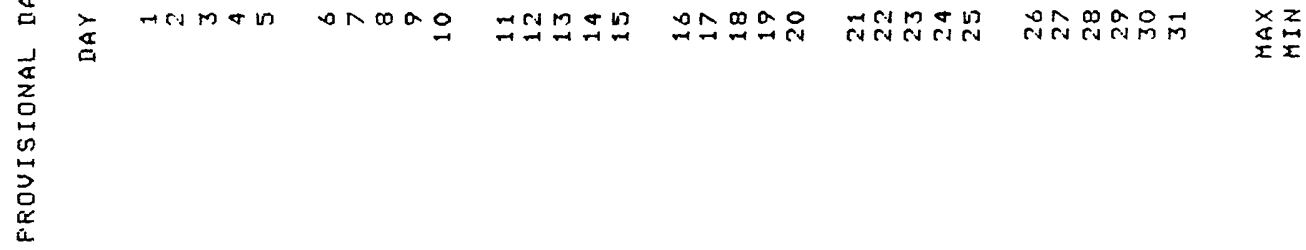




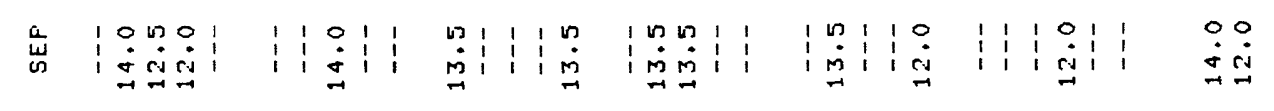

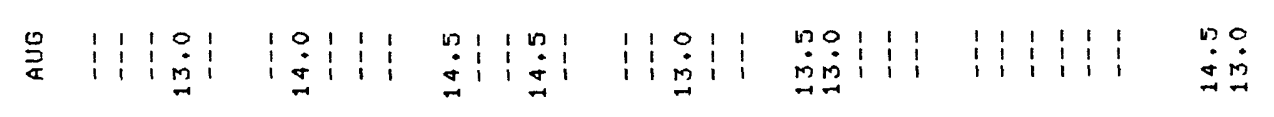

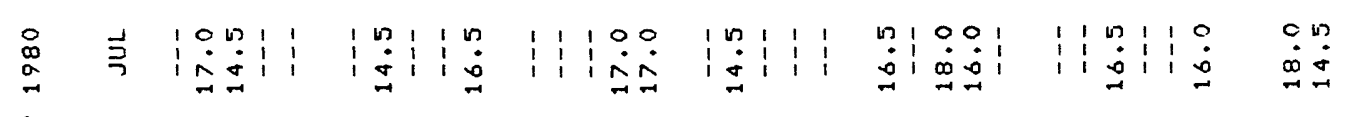

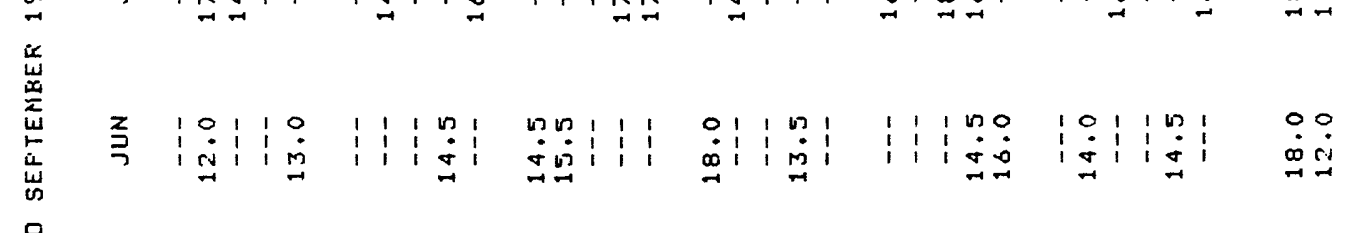
$\therefore$

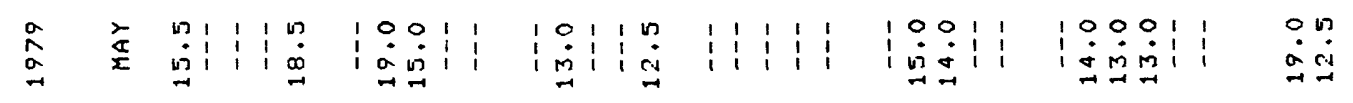

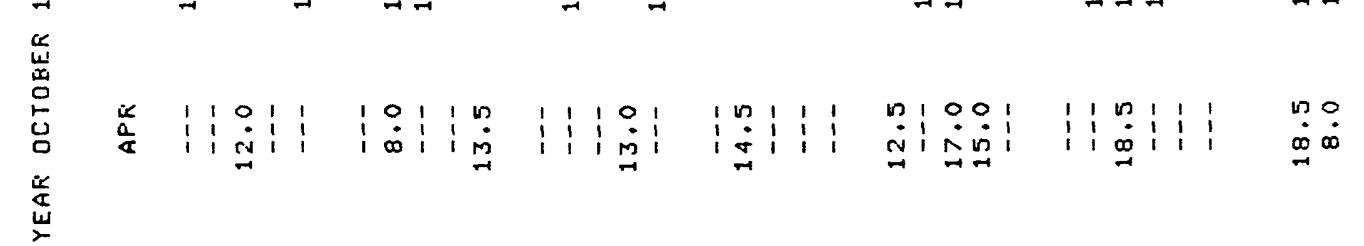

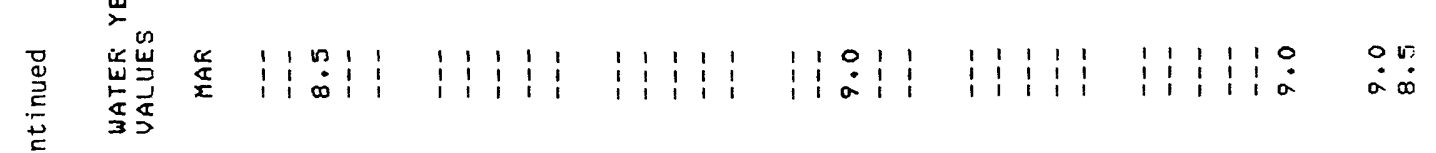

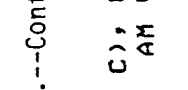

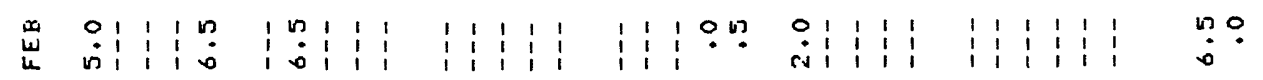

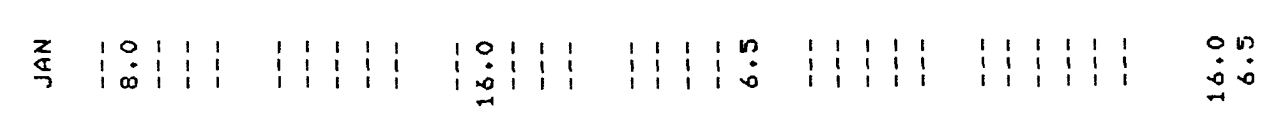
品 ż⿴囗十) 5

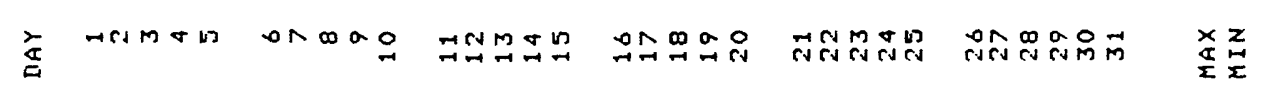


崫

苃

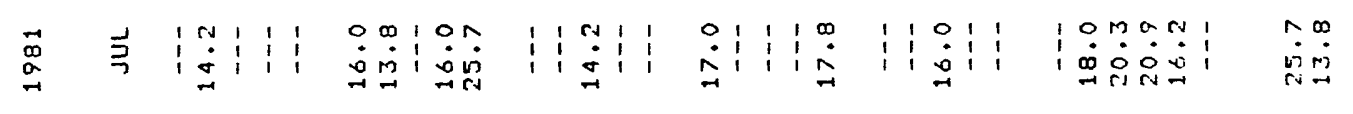

崖

웅

童童

迹

$\stackrel{\stackrel{c}{x}}{\check{w}}$

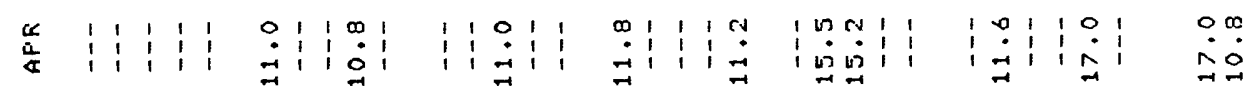

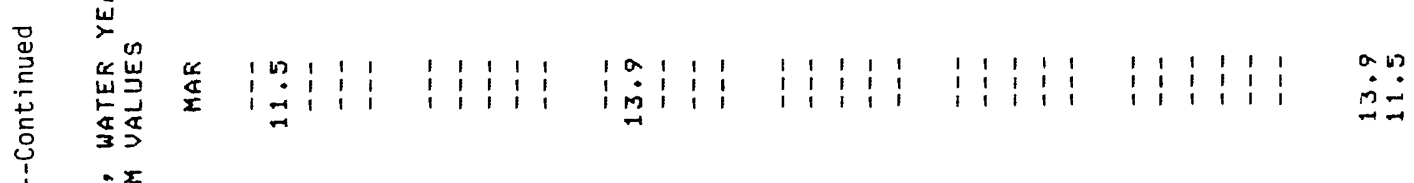

$\stackrel{0}{\Rightarrow} \quad \hat{0}^{\frac{\pi}{\sigma}}$

岕怠

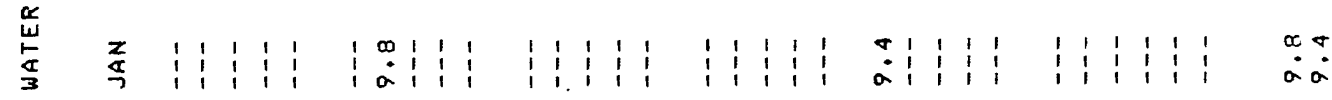

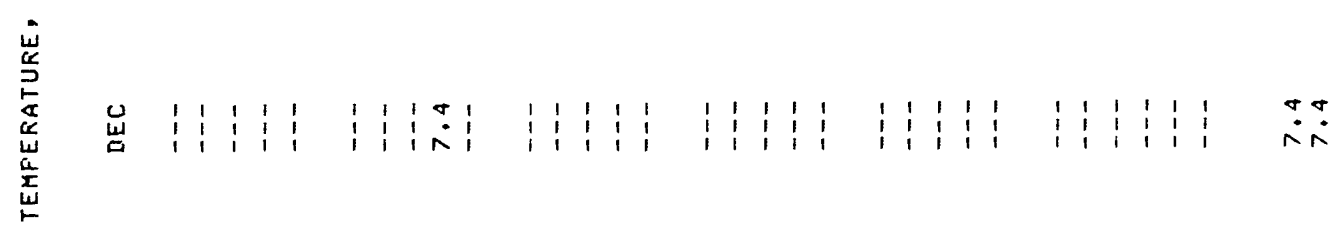

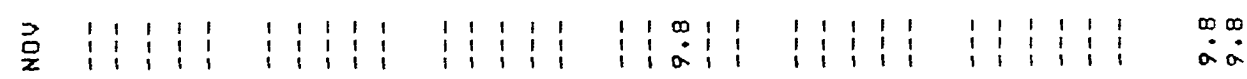

点

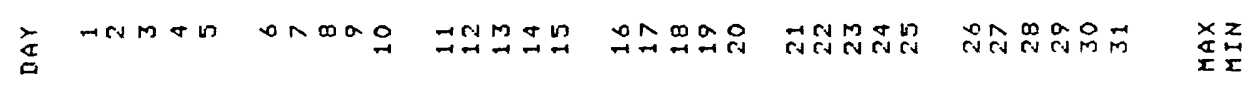




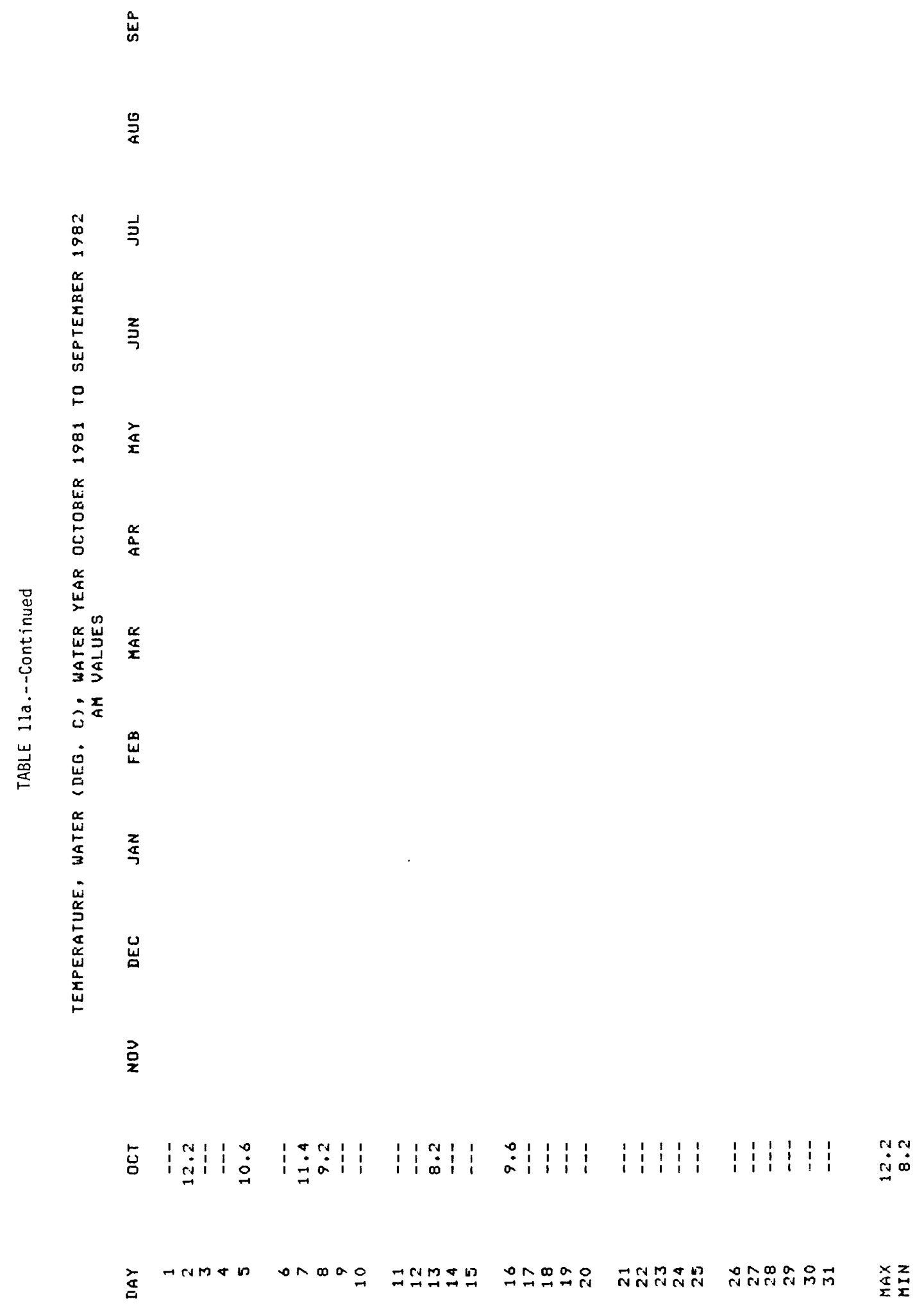




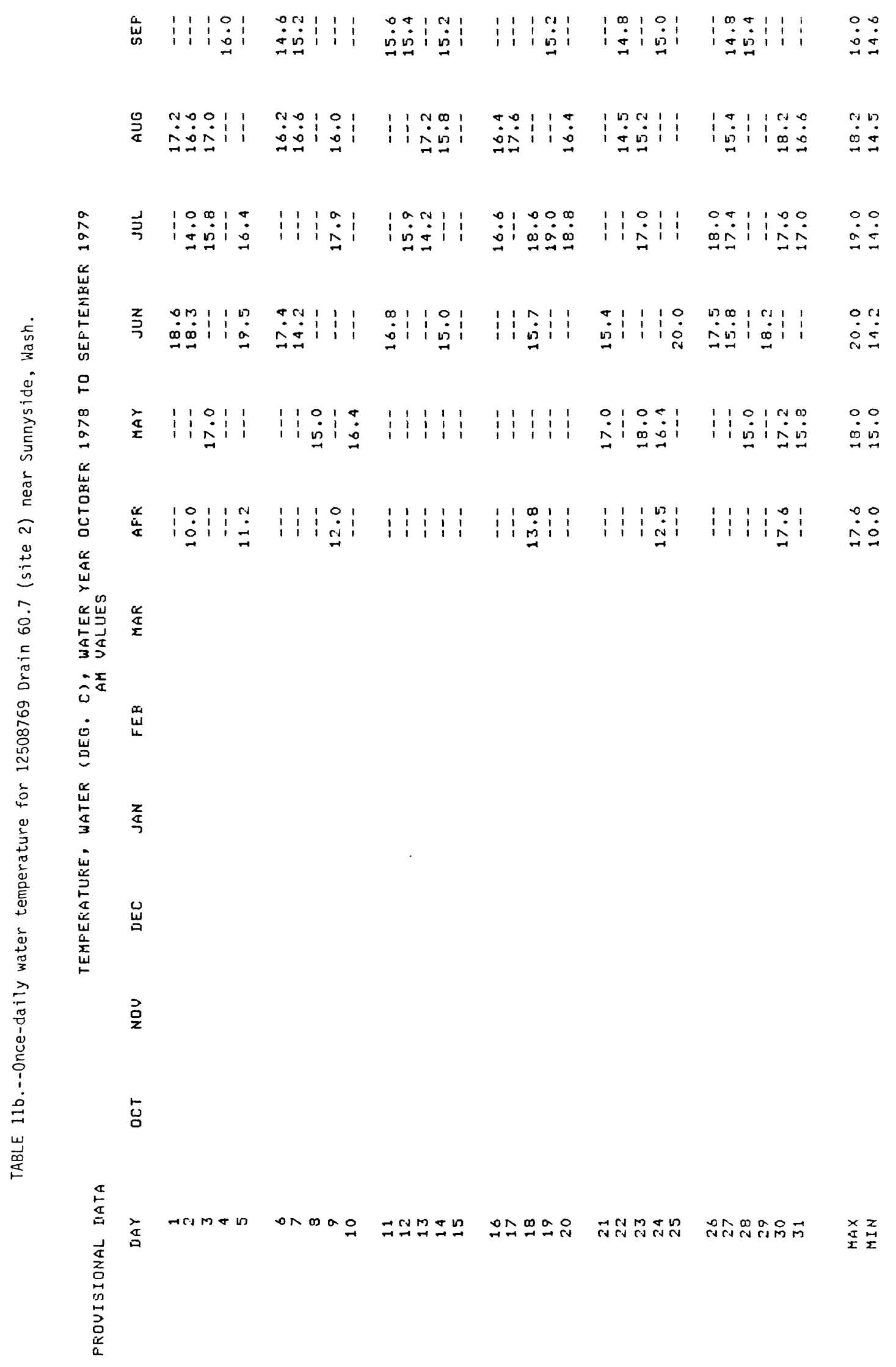




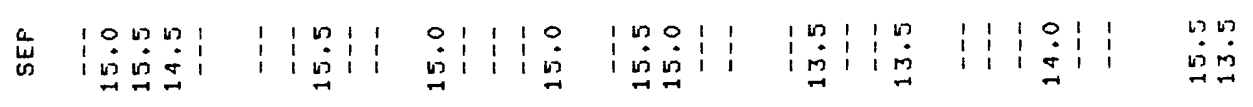
号

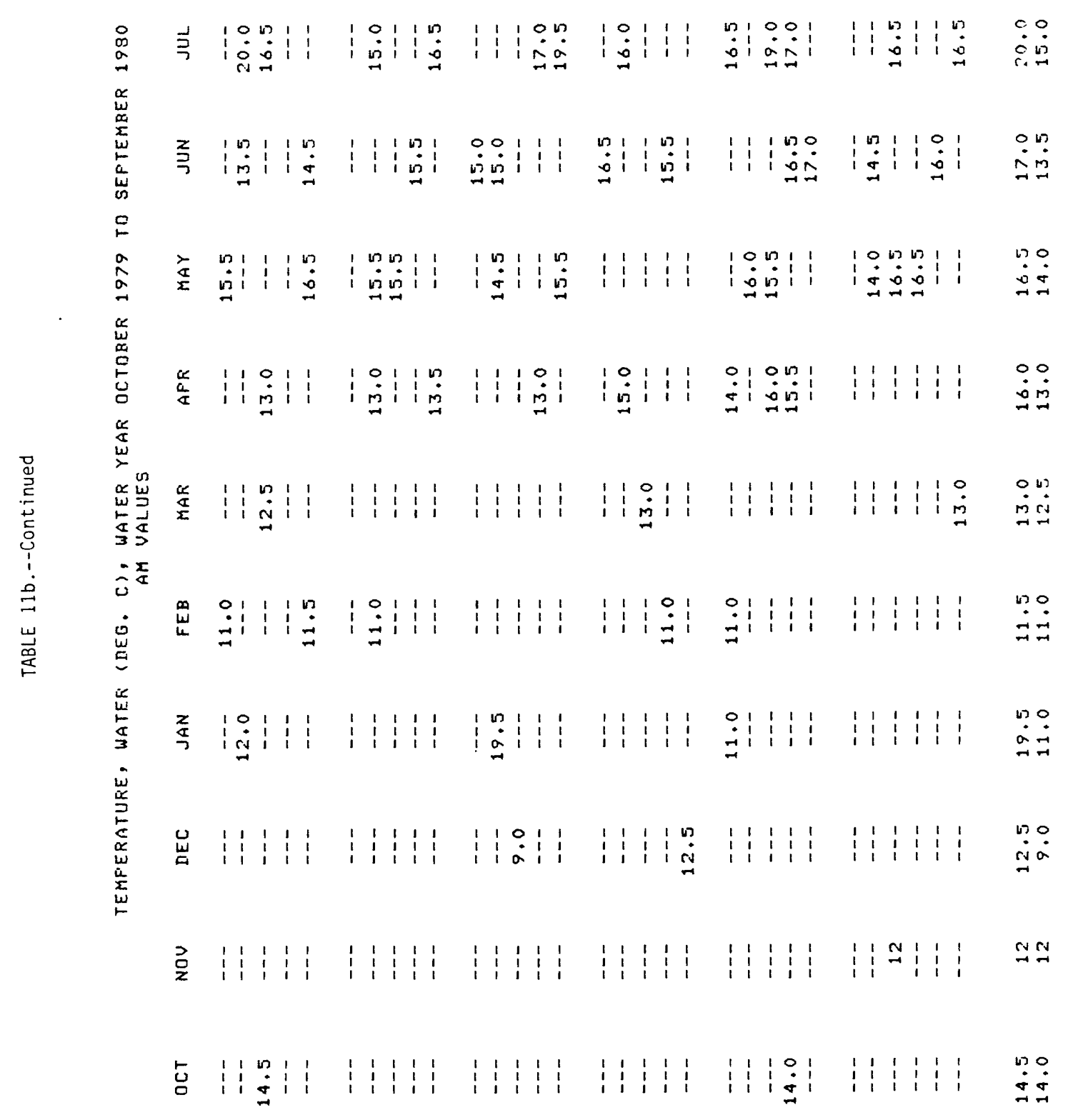

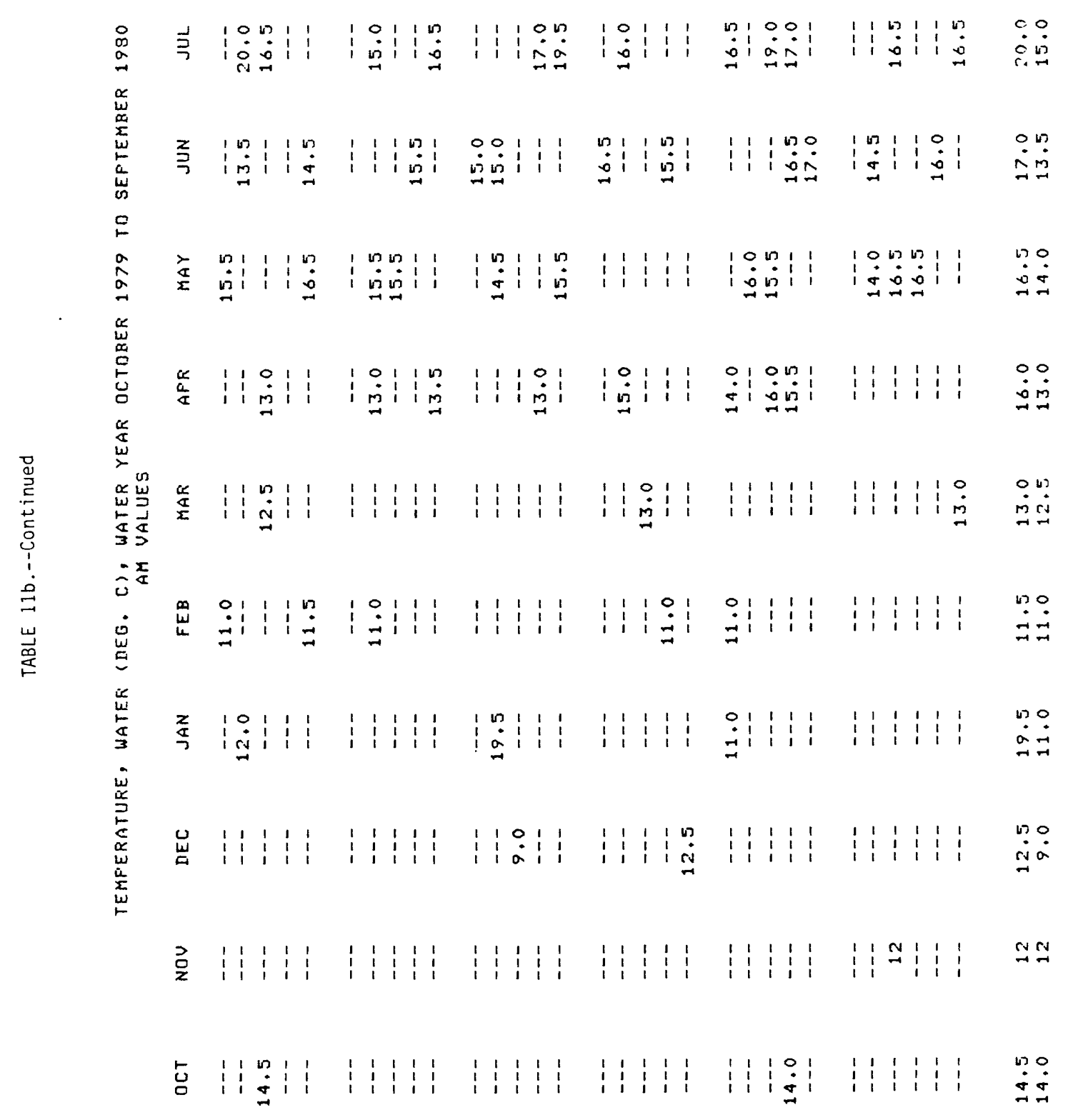

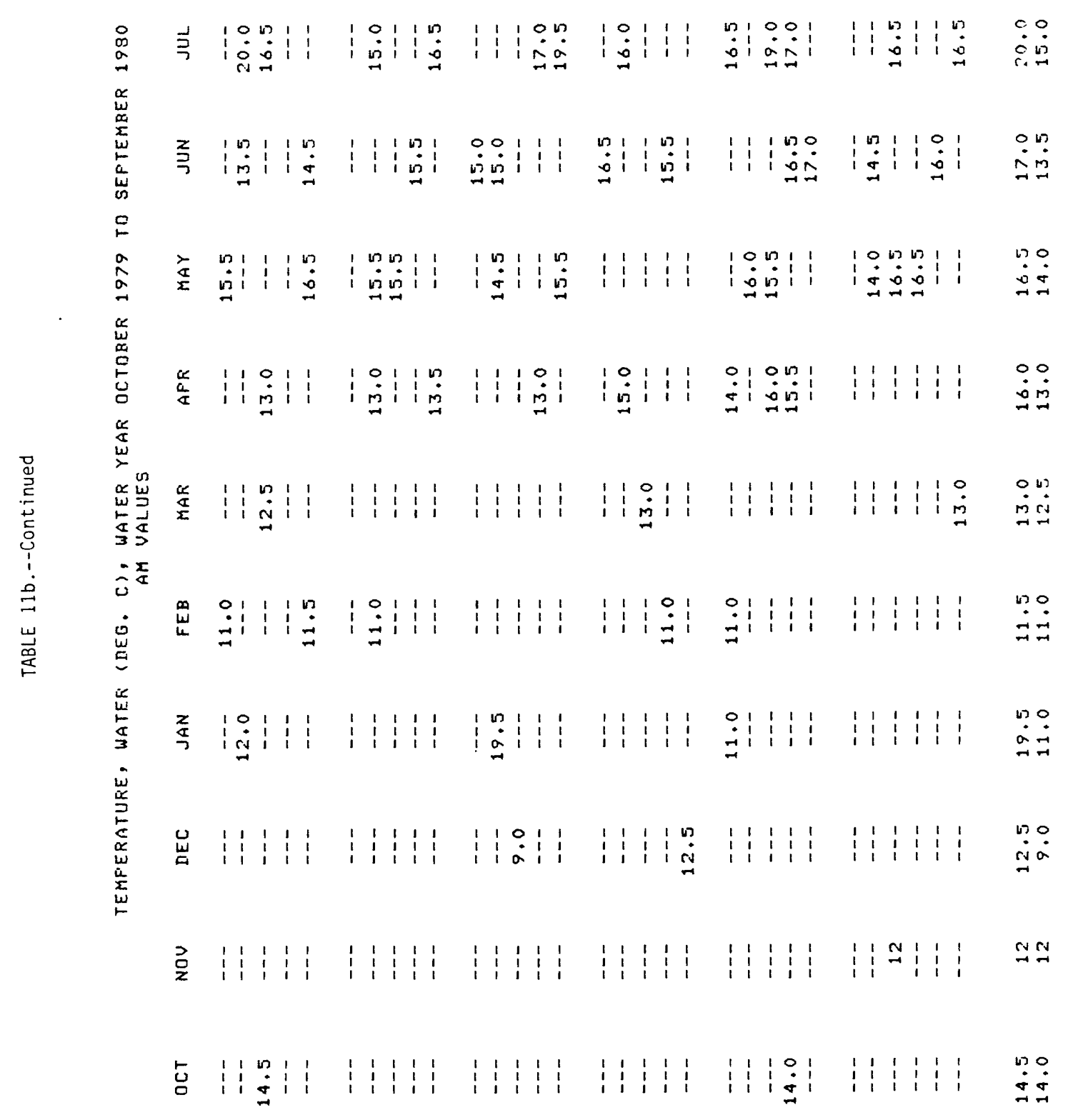

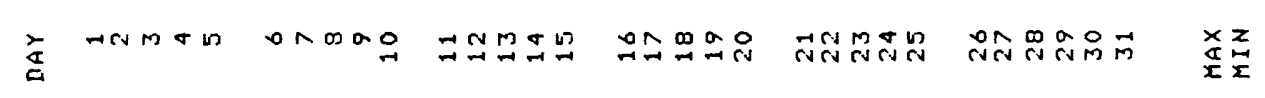




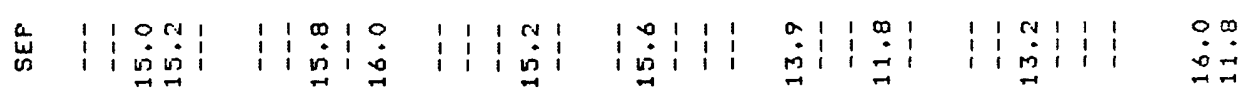
足

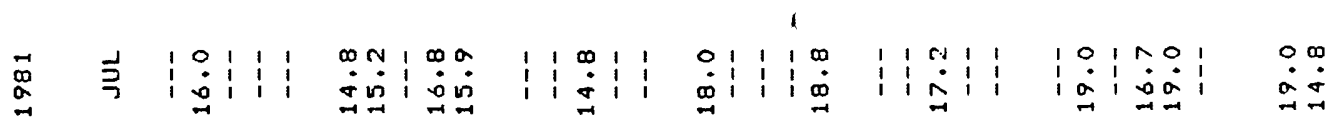

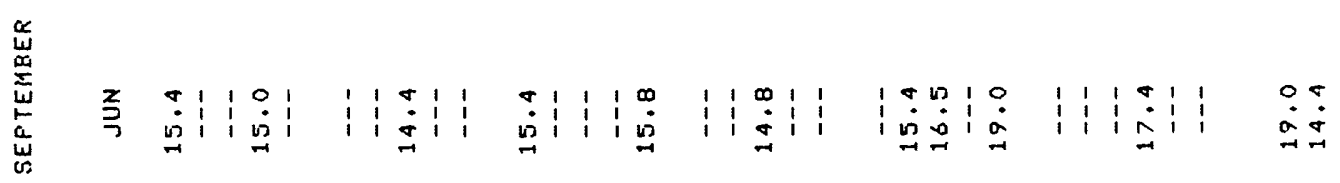
은

兽

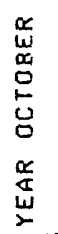

若

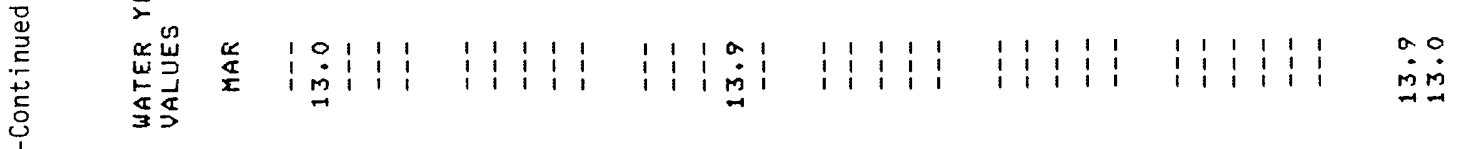
$i_{0}^{1} \quad 0^{\frac{\pi}{4}}$

岕

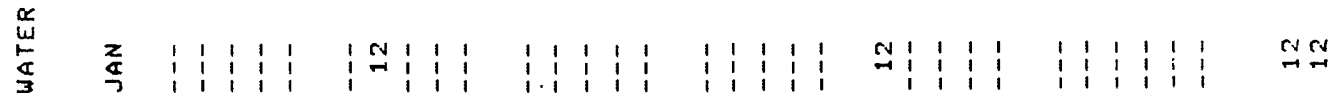

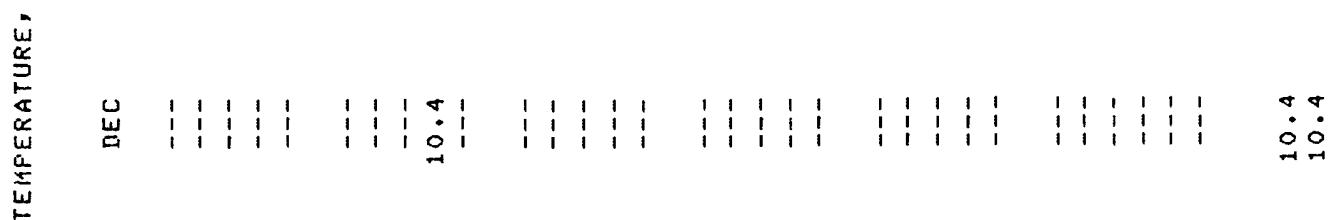
兴

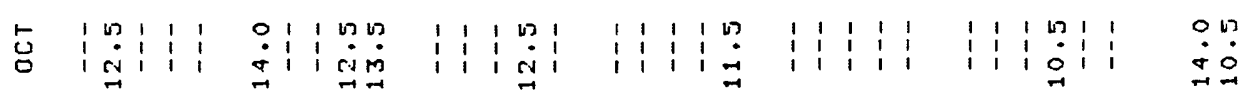

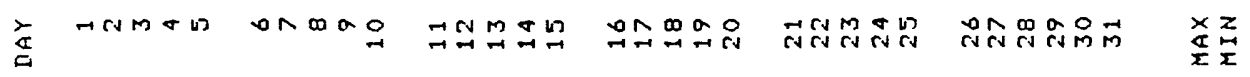




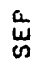

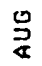

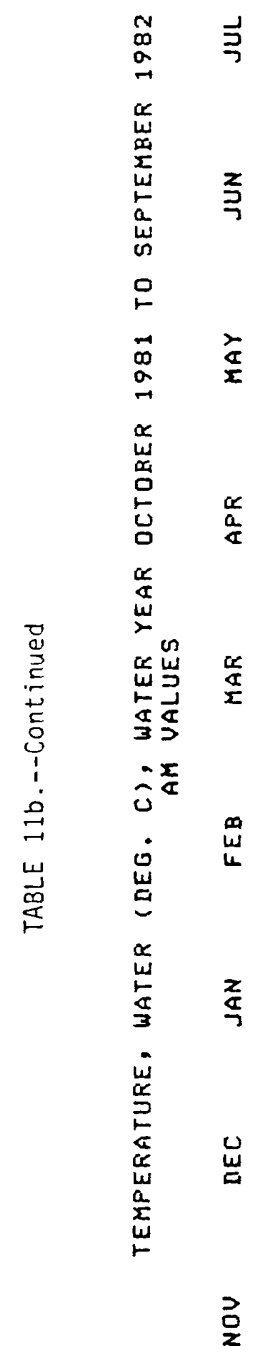

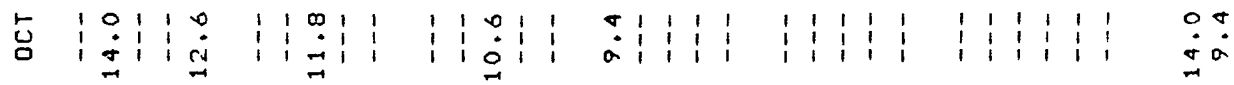

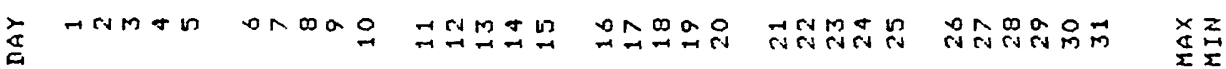




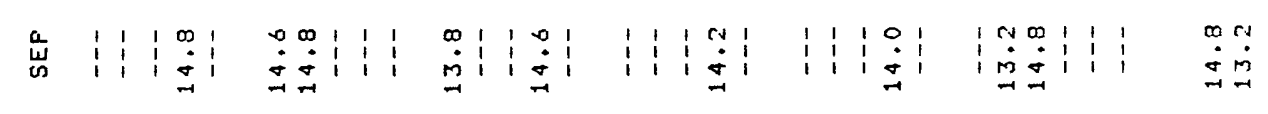

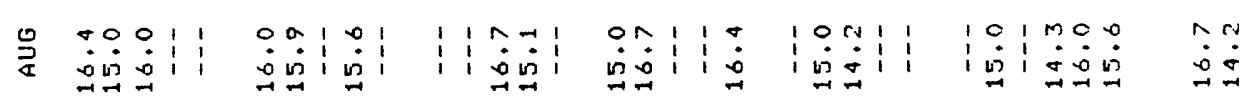

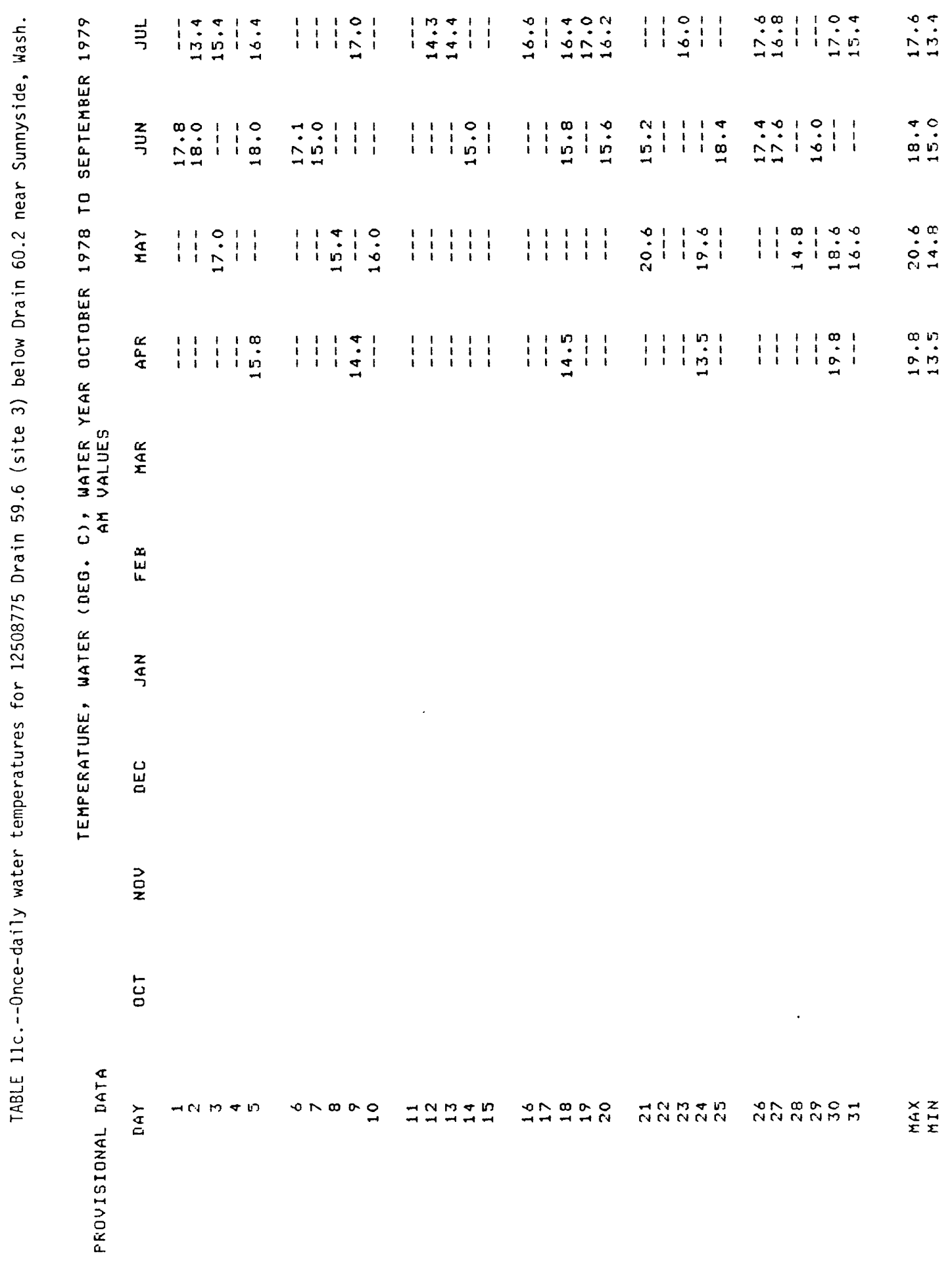


崫

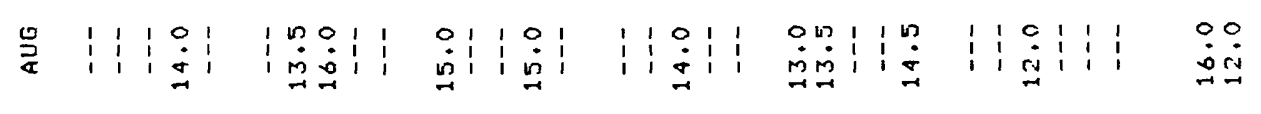

总

宏

$\stackrel{0}{1}$

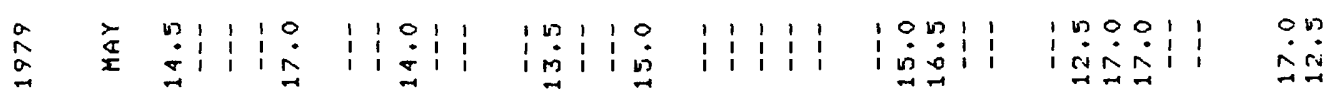

总

蒡

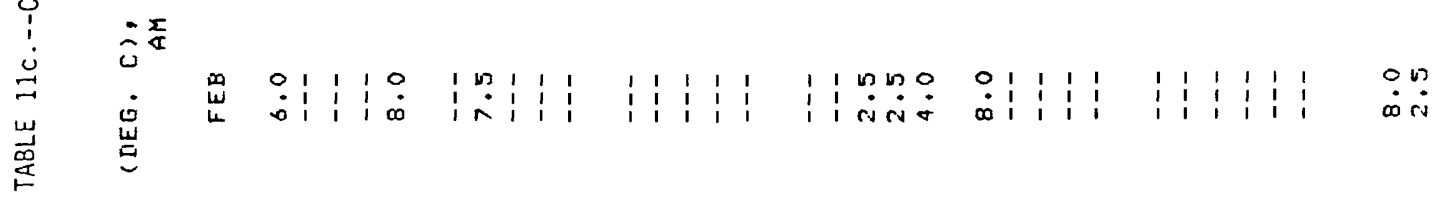

兽

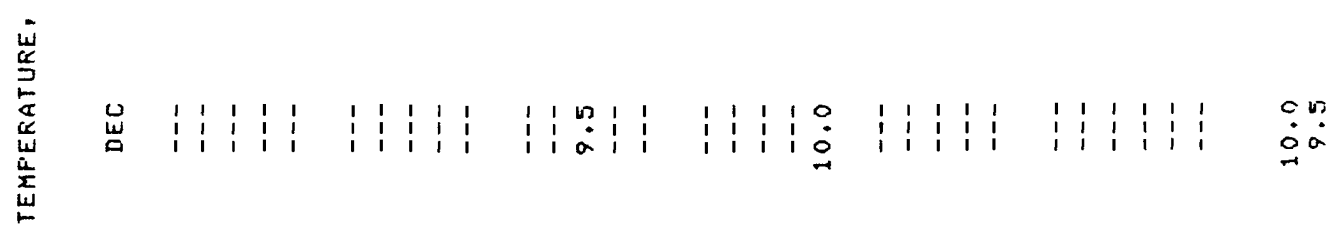

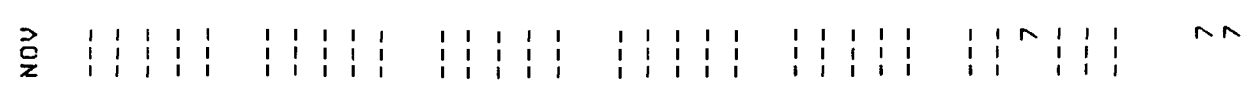

点

要 


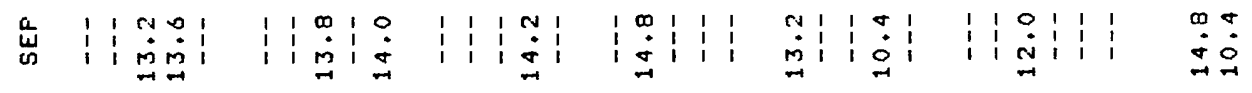

$$
\begin{aligned}
& \text { 号 } \\
& \text { 兽 }
\end{aligned}
$$

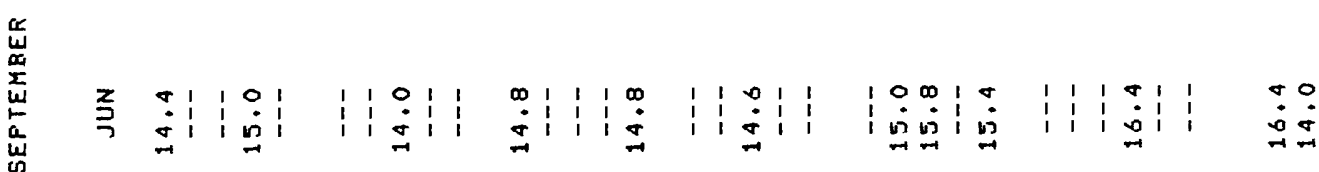

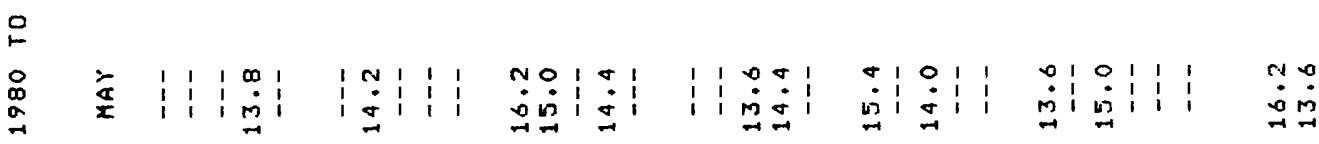

$$
\begin{aligned}
& \text { 崕 }
\end{aligned}
$$

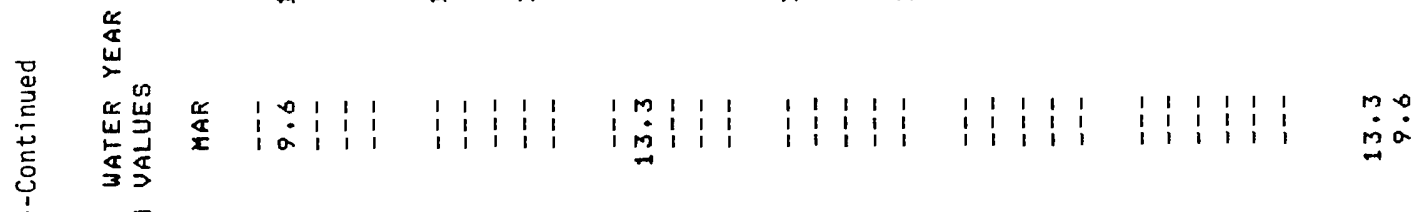

$$
\begin{aligned}
& \text { i. }
\end{aligned}
$$

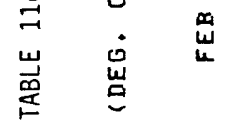

$$
\begin{aligned}
& \text { 营 }
\end{aligned}
$$

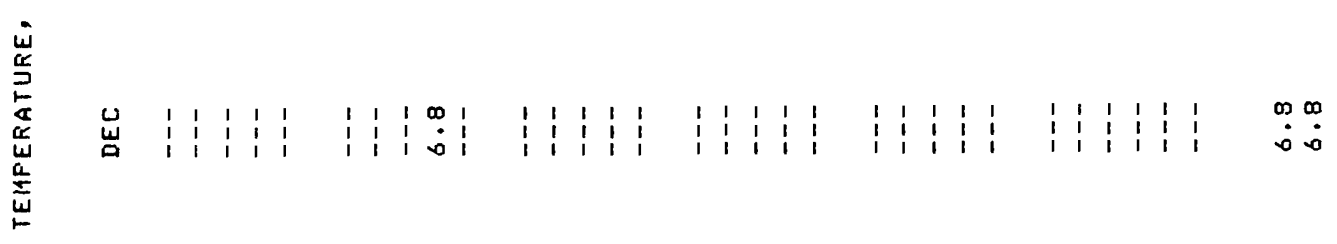

$$
\begin{aligned}
& \text { 룸 }
\end{aligned}
$$

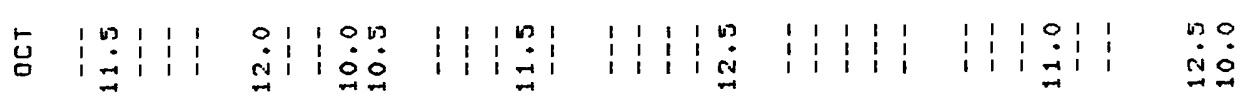

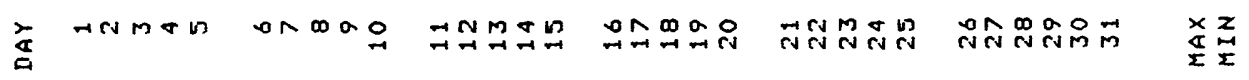


㟔

$\stackrel{9}{8}$

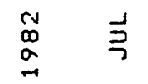

$$
\begin{aligned}
& \text { 䒴离 } \\
& \text { 웅 }
\end{aligned}
$$

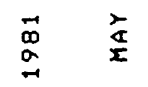

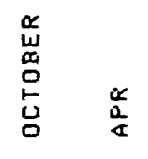

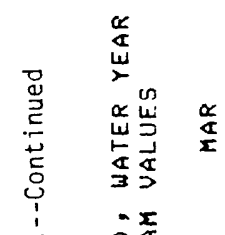

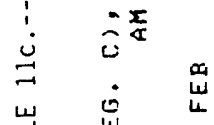

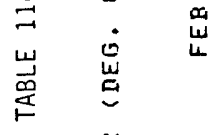

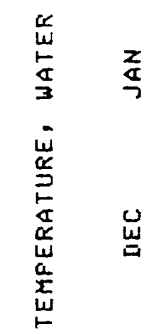

$$
\begin{aligned}
& \text { 훔 }
\end{aligned}
$$

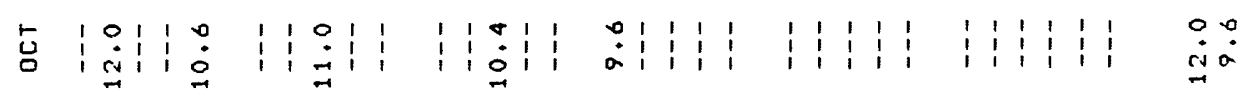

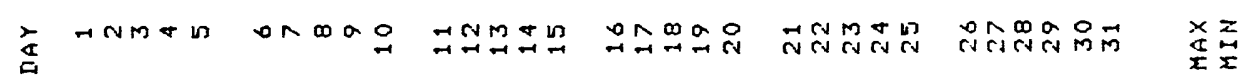


总

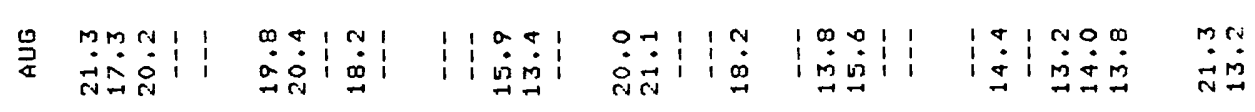

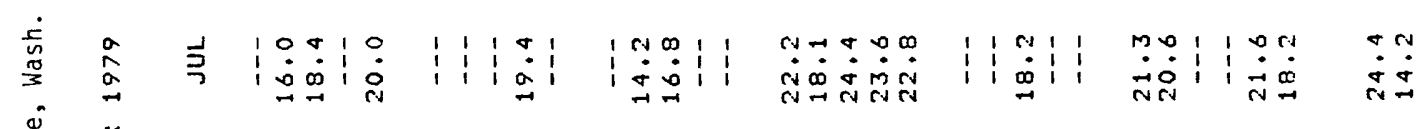

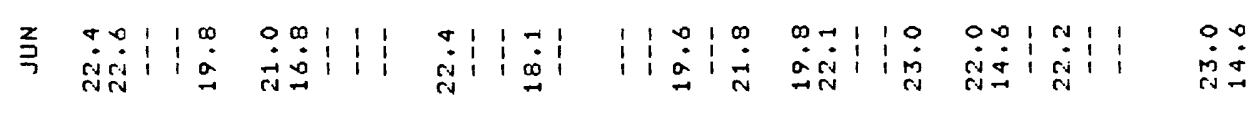

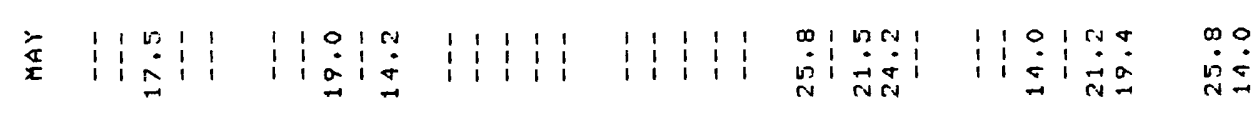

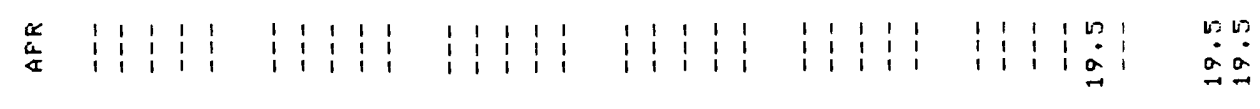

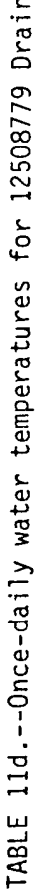

$\frac{0}{x}$

$\hat{i}=\frac{\pi}{4}$

i $\quad$ w

岂

$\underset{\substack{\frac{c}{w} \\ \frac{\pi}{3}}}{\stackrel{2}{4}}$

37

崖

㟔

吕

‘

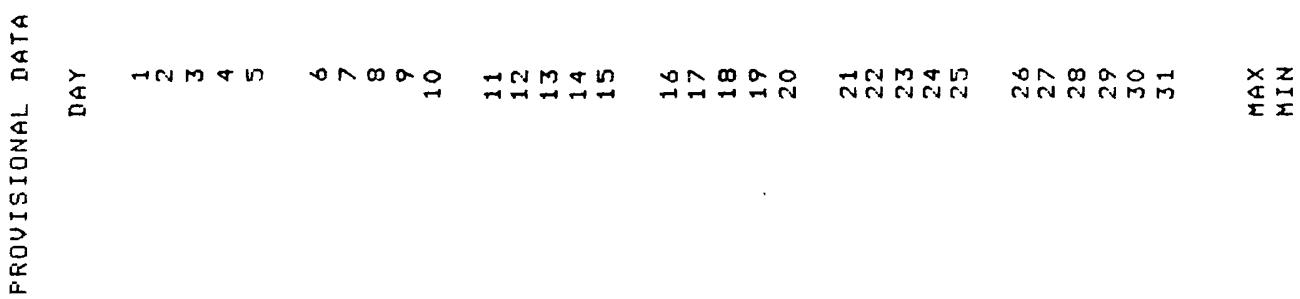




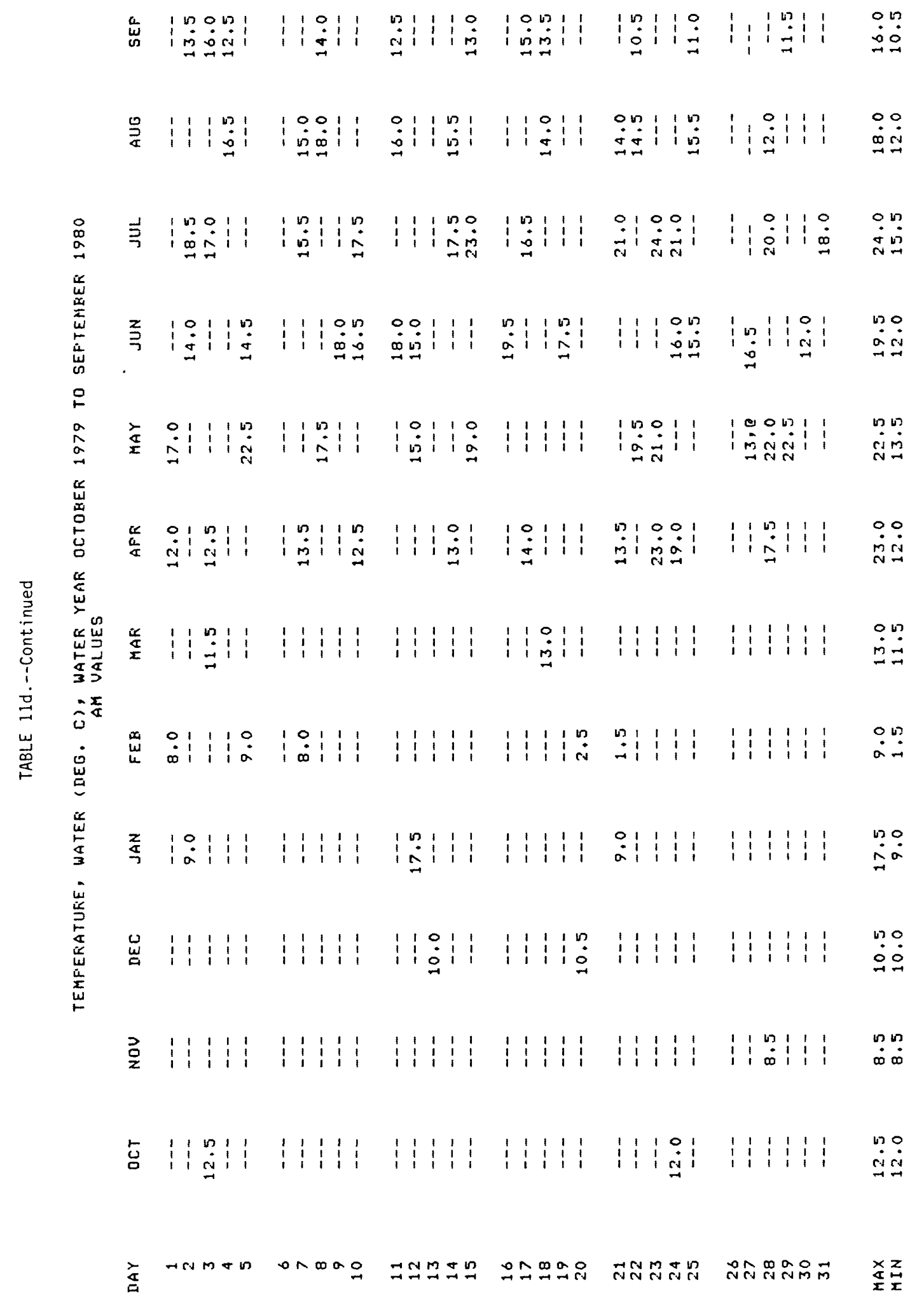




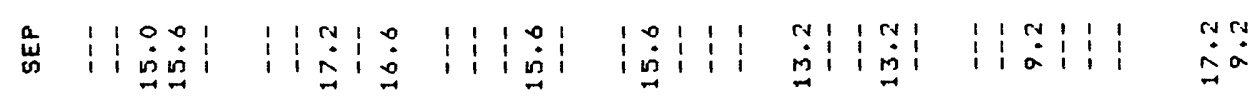

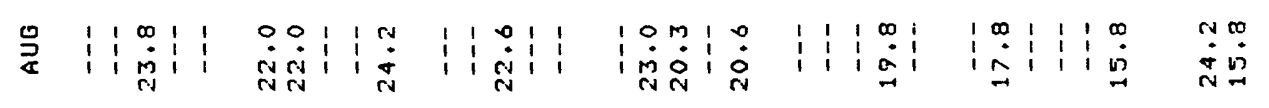

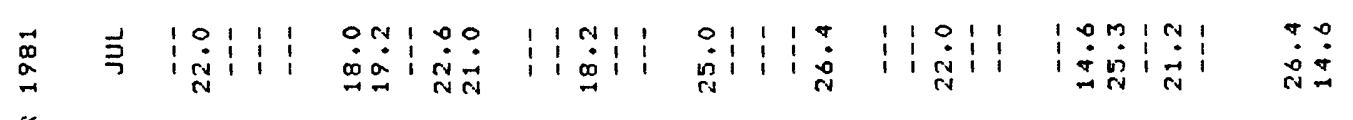

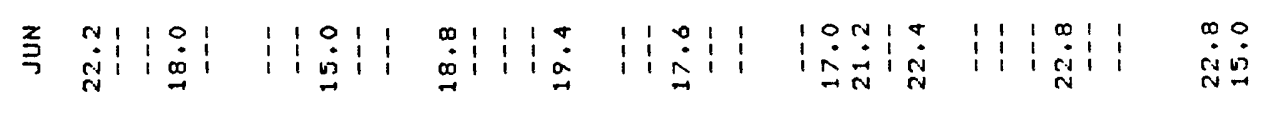

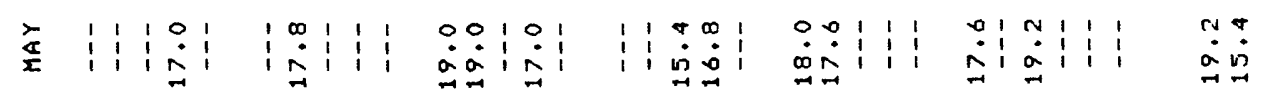
焉 彥 $\stackrel{m}{\stackrel{m}{u}}$

肴 㟒

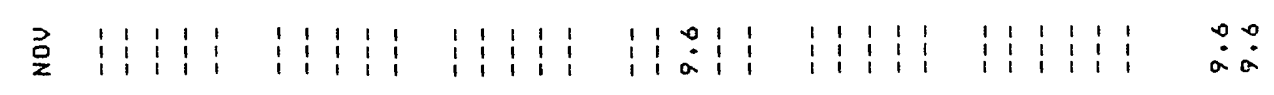

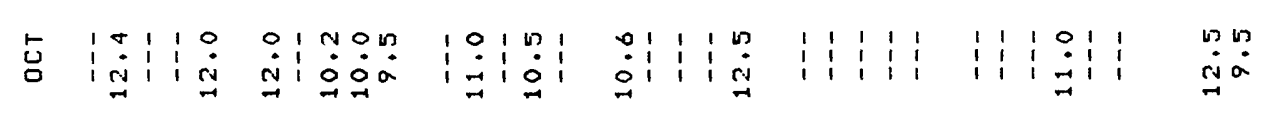

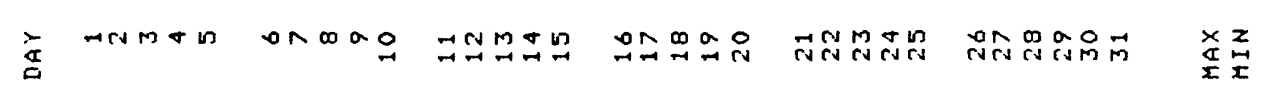


点

$\stackrel{9}{\triangleleft}$

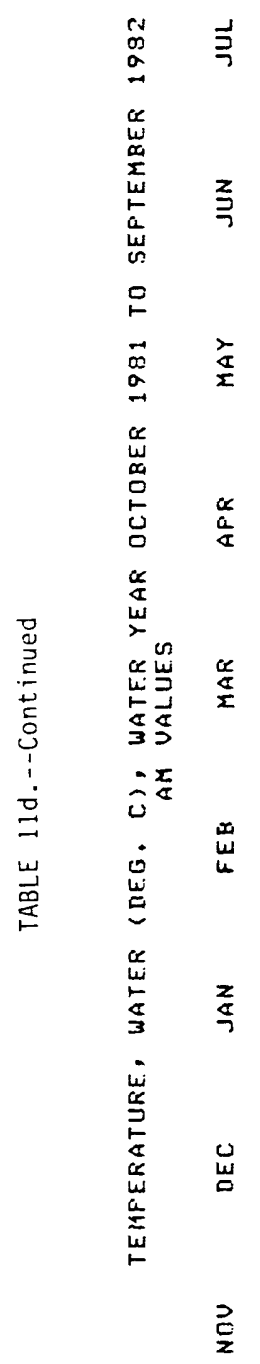

$\frac{3}{2}$

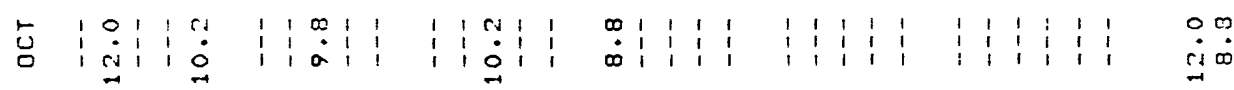

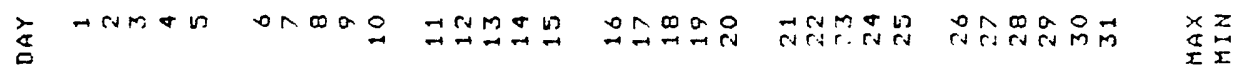


TABLE 12.--Analyses of miscellaneous suspended-sediment samples for sites in Drains 61.0, 50.7, 59.6, and 59.4 near Sunnyside, Wash.

$[E=$ estimated; $A=$ less than 0.005 tons $]$

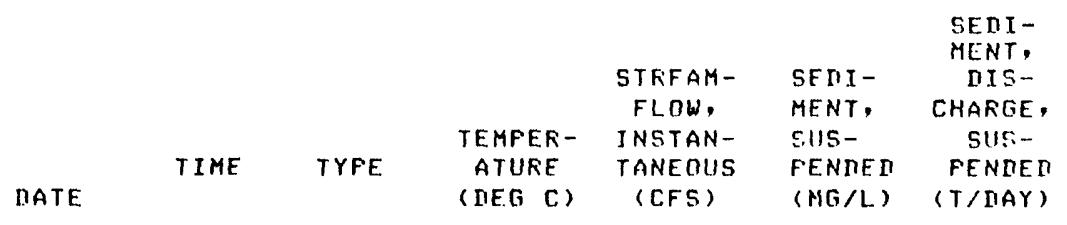

\section{SITE 11}

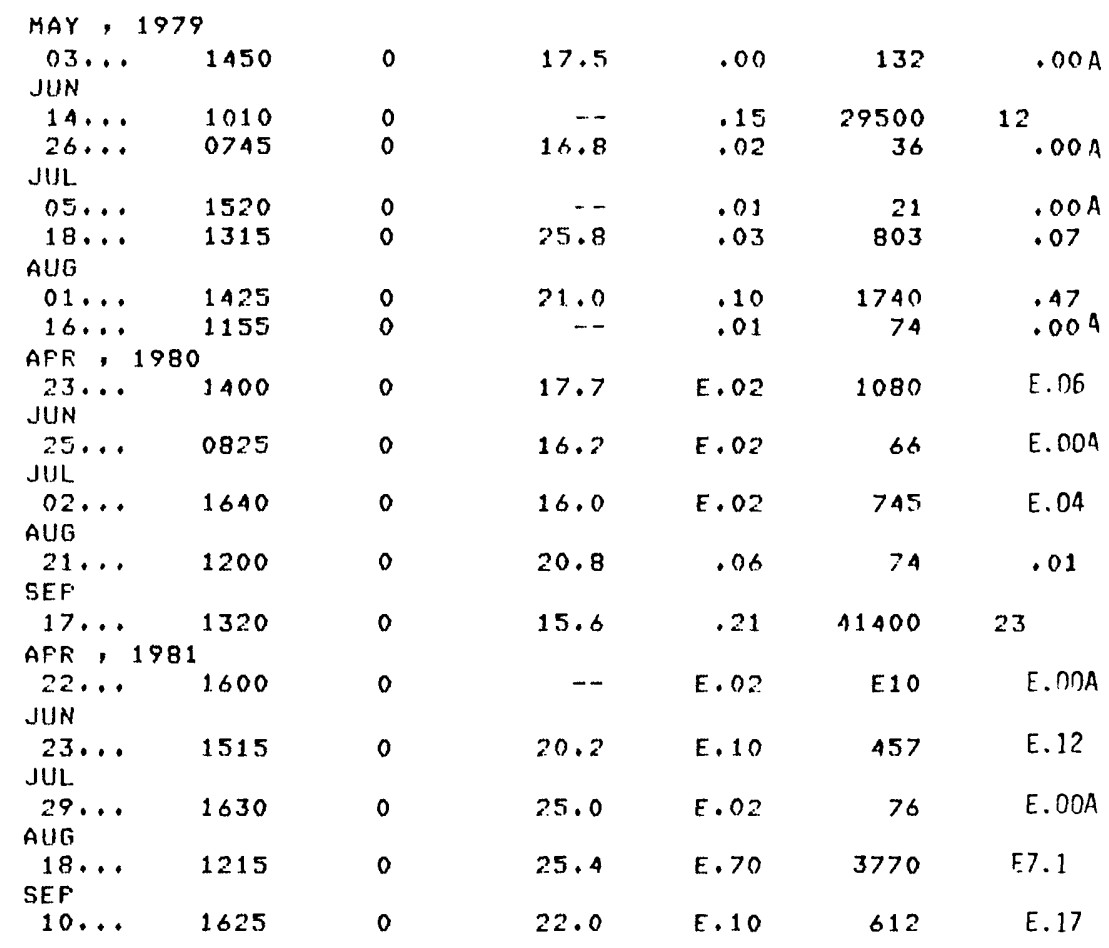

SITE 12

\begin{tabular}{|c|c|c|c|c|c|c|}
\hline \multicolumn{7}{|c|}{ JUN, 1979} \\
\hline $14 \ldots$ & 1450 & 0 & -- & .30 & 1340 & 1.1 \\
\hline $26 \ldots$ & 0745 & 0 & -- & .05 & 107 & .01 \\
\hline \multicolumn{7}{|l|}{ JUL } \\
\hline $05 \ldots$ & 1520 & 0 & -- & .03 & 21 & $.00 \mathrm{~A}$ \\
\hline $18 \ldots$ & 1315 & 0 & -- & .14 & 2670 & 1.0 \\
\hline \multicolumn{7}{|l|}{ AUG } \\
\hline $01 \ldots$ & 1425 & 0 & -- & .30 & 8140 & 6.6 \\
\hline $16 \ldots$ & 1155 & 0 & -- & .05 & 74 & $.00 \mathrm{~A}$ \\
\hline \multicolumn{7}{|c|}{ MAY, 1980} \\
\hline $07 \ldots$ & 1425 & 0 & 16.8 & $E .02$ & 212 & E. $\cap 1$ \\
\hline \multicolumn{7}{|l|}{ JUN } \\
\hline \multicolumn{7}{|l|}{ JIJL } \\
\hline $15 \ldots$ & 1050 & 0 & 18.0 & E. 26 & 499 & E. 35 \\
\hline \multicolumn{7}{|l|}{ AUG } \\
\hline $08 \ldots$ & 0952 & 0 & 17.8 & $E .02$ & 12 & E.OnA \\
\hline $21 \ldots$ & 1145 & 0 & 15.0 & E.02 & 394 & .02 \\
\hline \multicolumn{7}{|c|}{ JUN, 1981} \\
\hline $\begin{array}{l}23 \ldots \\
\text { JUL }\end{array}$ & 1517 & 0 & 21.8 & $E \cdot 15$ & 8860 & E3.6 \\
\hline $\begin{array}{l}29 \\
\text { AUG }\end{array}$ & 1625 & 0 & 20.1 & $E \cdot 10$ & 11 & E.OOA \\
\hline $\begin{array}{r}18 \ldots \\
\text { SEF }\end{array}$ & 1220 & 0 & 19.4 & E.01 & 2 & E.OกA \\
\hline $10 \ldots$ & 1630 & 0 & 20.6 & $E .03$ & 2 & E.004 \\
\hline
\end{tabular}


TABLE 12.--Continued

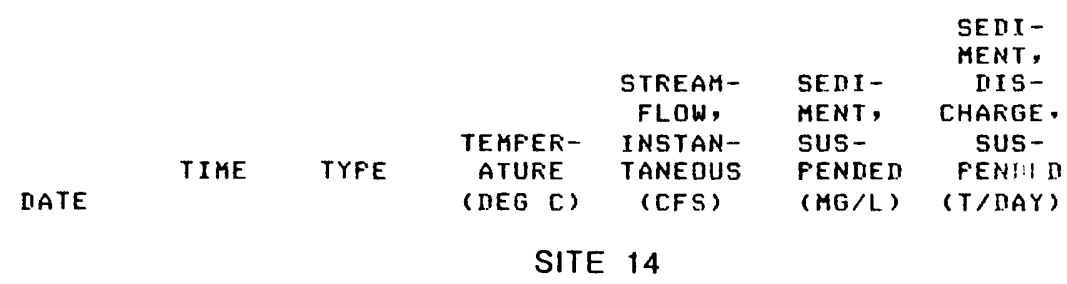

12508771

- DRAIN 60.0 AT SLI RII. NR SUNNYSIDE, WASH. (LAT 462140 LDNG 11956 47)

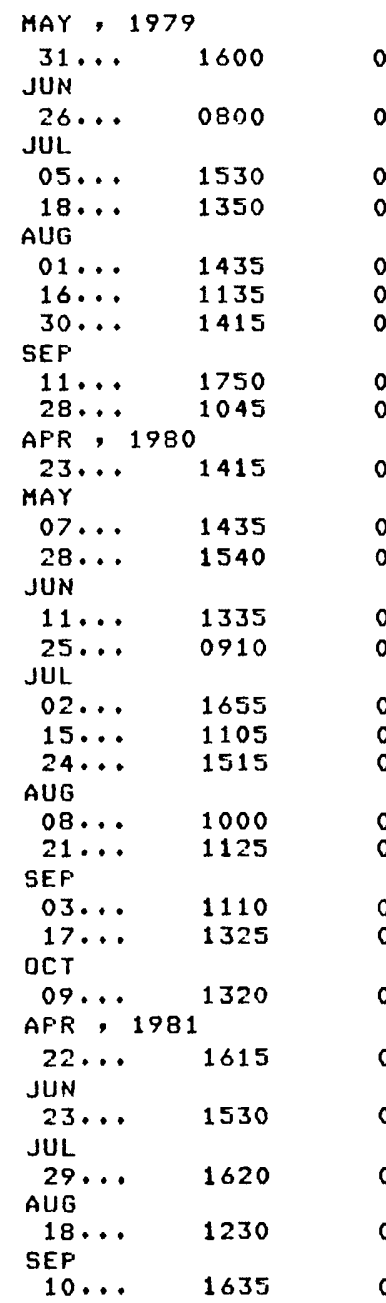

\begin{tabular}{|c|c|c|c|}
\hline-- & .50 & 1030 & 1.4 \\
\hline 17.4 & 1.4 & 472 & 1.8 \\
\hline 21.9 & 1.0 & 72 & .19 \\
\hline $22 \cdot 2$ & .68 & 13500 & 25 \\
\hline $\begin{array}{l}19.8 \\
15.2 \\
15.6\end{array}$ & $\begin{array}{r}.54 \\
.30 \\
.28\end{array}$ & $\begin{array}{r}44 \\
851 \\
13\end{array}$ & $\begin{array}{l}.06 \\
.69 \\
.00 \mathrm{~A}\end{array}$ \\
\hline $\begin{array}{r}14.0 \\
--\end{array}$ & $\begin{array}{l}.14 \\
.10\end{array}$ & $\begin{array}{l}2 \\
2\end{array}$ & $\begin{array}{l}.00 \mathrm{~A} \\
.00 \mathrm{~A}\end{array}$ \\
\hline 17.0 & $E .02$ & 38 & E.DOA \\
\hline 22.4 & .27 & 1560 & $1 \cdot 1$ \\
\hline 19.4 & .34 & 209 & .19 \\
\hline $\begin{array}{l}18.4 \\
14.6\end{array}$ & $\begin{array}{l}.10 \\
.59\end{array}$ & $\begin{array}{r}52 \\
E 2000\end{array}$ & $E 3.2$ \\
\hline $\begin{array}{l}21.0 \\
19.4 \\
23.4\end{array}$ & $\begin{array}{c}.41 \\
1.1 \\
.73\end{array}$ & $\begin{array}{l}222 \\
149 \\
144\end{array}$ & $\begin{array}{l}.25 \\
.44 \\
.28\end{array}$ \\
\hline $\begin{array}{l}15.9 \\
16.8\end{array}$ & $\begin{array}{l}.31 \\
.85\end{array}$ & $\begin{array}{r}53 \\
626\end{array}$ & $\begin{array}{l}.04 \\
1.4\end{array}$ \\
\hline $\begin{array}{l}14.4 \\
16.2\end{array}$ & $\begin{array}{l}.56 \\
.28\end{array}$ & $\begin{array}{r}2310 \\
125\end{array}$ & $\begin{array}{l}3.5 \\
.09\end{array}$ \\
\hline 15.5 & .96 & 1380 & 3.6 \\
\hline 14.2 & $E .07$ & 46 & E.n1 \\
\hline 17.1 & E. 30 & 4220 & E3. 4 \\
\hline 21.1 & $E \cdot 30$ & 944 & E.76 \\
\hline 22.6 & $E .07$ & 287 & E.05 \\
\hline $18 \cdot 2$ & $E .05$ & 19 & E.COA \\
\hline
\end{tabular}


TABLE 12.--Continued

\begin{tabular}{|c|c|c|c|c|c|c|}
\hline \multicolumn{7}{|r|}{ SEIII- } \\
\hline \multirow[b]{3}{*}{ IATE } & \multirow{3}{*}{ TIME } & \multirow{3}{*}{ TYFE } & \multirow{3}{*}{$\begin{array}{l}\text { TEMFEF - } \\
\text { ATUIFE } \\
\text { (IIEG C ) }\end{array}$} & $\begin{array}{l}\text { STFEAM- } \\
\text { FLOW, }\end{array}$ & $\begin{array}{l}\text { SF UI - } \\
\text { MENT, }\end{array}$ & $\begin{array}{l}\text { IIIS- } \\
\text { CHAEGE, }\end{array}$ \\
\hline & & & & $\begin{array}{l}\text { INST AHH- } \\
\text { THNE OUS }\end{array}$ & $\begin{array}{l}\text { SUS- } \\
\text { FENIIE II }\end{array}$ & $\begin{array}{l}\text { SUS - } \\
\text { FEU. II }\end{array}$ \\
\hline & & & & (CFS) & $(M G / L)$ & $(T /[A A Y)$ \\
\hline & & & SI & 15 & & \\
\hline
\end{tabular}

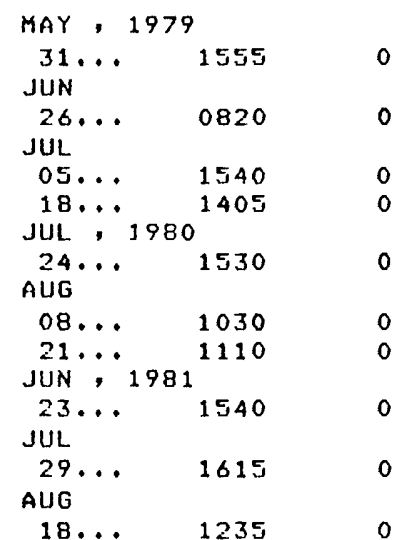

$\begin{array}{rrrl}16.5 & .10 & 48 & .01 \\ 19.4 & .06 & 10 & .00 A \\ 26.2 & .06 & 13 & .00 A \\ 29.0 & .26 & 339 & .24 \\ 26.6 & E .15 & 28 & E .01 \\ 18.4 & .14 & 155 & .06 \\ 18.2 & E .05 & 17 & E .00 A \\ 25.9 & E .04 & 48 & E .01 \\ 26.8 & E .05 & 27 & E .00 A \\ 25.7 & E .05 & 191 & E .03\end{array}$


TABLE 12.--Continued

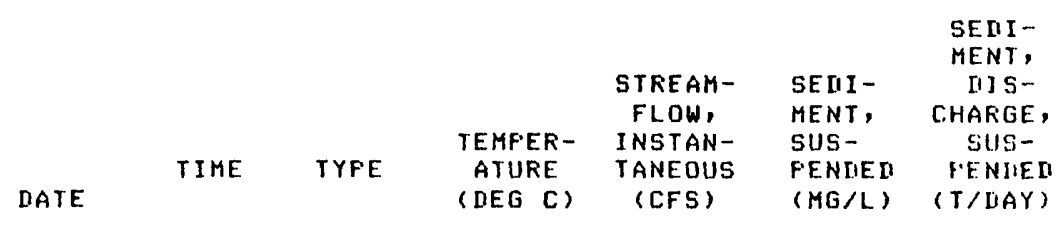

\section{SITE 16}

$14 \ldots \quad 0950$

$26 \ldots .0830$

$$
\text { JUL }
$$$$
05 . . .1550
$$$$
18 \ldots 1420
$$$$
\text { AUG }
$$

$01 \ldots \quad 1440$

$16 \ldots 1100$

$30 \ldots 1445$

MAY, 1980

$07 \ldots \quad 1455$

$28 \ldots \quad 1555$

JUN

$11 \ldots \quad 1345$

$25 . \ldots \quad 0935$

JUL

$02 \ldots \quad 1720$

$15 \ldots \quad 1150$

$24 \ldots \quad 1535$

AUG

08... 1040

SEF

$17 \ldots \quad 1335$

OCT

$09 \ldots \quad 1245$

APR , 1981

$22 \ldots \quad 1625$

JUN

$23 \ldots .1550$

JUL

29... 1605

AUG

$18 . . . \quad 1240$

SEF

$10 \ldots 1645$

$$
33 .
$$$$
19.0
$$$$
33.0
$$$$
22.8
$$$$
22.8
$$

28.2

23.2
24.0

19.0

15.8

26.0

19.8

28.0

18.2

21.0

13.6

--

18.5

25.3

26.4

22.2

SITE 13

12508770

- IRAIN 59.6 AT SLI RI. NR

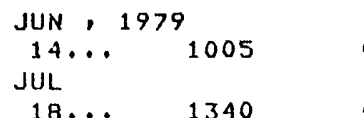

$18 \ldots \quad 1340$
0

$\begin{array}{rrrrr}0 & -- & .10 & 272 & .07 \\ 0 & 32.8 & .01 & 28100 & .77\end{array}$

32.8

$\begin{array}{rrrrr}0 & -1 & .10 & 272 & .07 \\ 0 & 32.8 & .01 & 28100 & .77\end{array}$

$\begin{array}{rl}7560 & 6.1 \\ 563 & .91 \\ 126 & .00 \mathrm{~A} \\ 451 & .23 \\ 50 & .00 \mathrm{~A} \\ 100 & .10 \\ 100 & .18\end{array}$

E. .05

.32
$E .40$

E. 10

E.60

E.05

E. 62

E.06

E. 02

.23

.44

E. 10

E. 08

E. 50

E.05

E. 07

55
195

181 E.05

31 E.05

277

E.04

26 E.04

160 E.03

$380 \quad E .02$

$768 \quad .48$

$69 \quad .08$

49 E.01

1980 E.4.3

$4420 \quad E 6.0$

$950 \quad E .13$

419 E.08

SITE 13

WASH. (LAT 462140 LONG 1195655 ) 
TABLE 12.--Continued

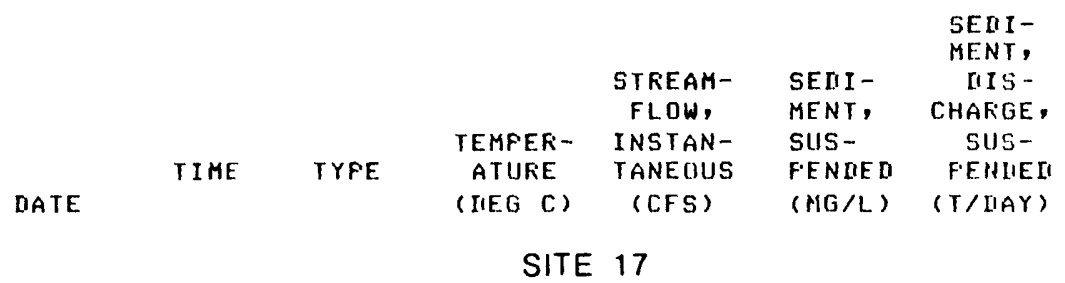

\begin{tabular}{|c|c|c|c|c|c|c|}
\hline $\begin{array}{l}\text { MAY } \\
31 \ldots \\
\text { JUN }\end{array}$ & 1545 & 0 & 18.5 & .80 & 2800 & 6.0 \\
\hline $14 \ldots$ & 0945 & 0 & -- & .50 & 2660 & 3.6 \\
\hline $26 \ldots$ & 0900 & 0 & 21.2 & .73 & 52900 & 104 \\
\hline \multicolumn{7}{|l|}{ JUL } \\
\hline $05 \ldots$ & 1600 & 0 & 28.4 & .37 & 9920 & 9.9 \\
\hline $18 \ldots$ & 1440 & 0 & 29.6 & .29 & 5950 & 4.7 \\
\hline \multicolumn{7}{|l|}{ AUG } \\
\hline $01 \ldots$ & 1450 & 0 & 25.2 & .88 & 974 & 2.3 \\
\hline $16 \ldots$ & 1030 & 0 & 20.6 & .45 & 955 & 1.2 \\
\hline $30 \ldots$ & 1450 & 0 & -- & .60 & 39 & .06 \\
\hline \multicolumn{7}{|c|}{ MAY, 1980} \\
\hline $07 \ldots$ & 1515 & 0 & 20.7 & E. 10 & 4770 & E1.3 \\
\hline \multicolumn{6}{|l|}{ JUN } & 3.8 \\
\hline $11 \ldots$ & 1355 & 0 & 22.0 & E. 10 & 815 & E.22 \\
\hline $25 \ldots$ & 0950 & 0 & 21.8 & .41 & 302 & .33 \\
\hline \multicolumn{7}{|l|}{ JUL } \\
\hline $02 \ldots$ & 1720 & 0 & 21.8 & E.50 & 305 & E.41 \\
\hline $15 \ldots$ & 1200 & 0 & 22.8 & .48 & 37 & .05 \\
\hline $\begin{array}{l}24 \ldots \\
\text { AUG }\end{array}$ & 1545 & & 26.0 & E.05 & 20 & $E . O N A$ \\
\hline $08 \ldots$ & 1050 & 0 & 18.6 & .05 & 4 & $.00 \mathrm{~A}$ \\
\hline$\underset{S E F}{21} \cdots$ & 1055 & 0 & 18.8 & .06 & 0 & $.00^{n}$ \\
\hline $03 \ldots$ & 1050 & 0 & 16.6 & .06 & 1 & $.00^{A}$ \\
\hline $\begin{array}{c}17 \ldots \\
\text { OC, }\end{array}$ & 1345 & 0 & 24.0 & .07 & 528 & .10 \\
\hline $\begin{array}{l}09 . \cdots \\
A F R,\end{array}$ & 1235 & 0 & 14.7 & $E .03$ & 15 & E. $\cap \cap A$ \\
\hline $22 \ldots$ & 1630 & 0 & 17.0 & E.05 & 1130 & E.15 \\
\hline $\begin{array}{l}\text { JUN } \\
23 \ldots\end{array}$ & & & & & & \\
\hline JUL & 1555 & 0 & 24.0 & $E .35$ & 66900 & E63 \\
\hline $29 \ldots$ & 1555 & 0 & 24.9 & $E .42$ & 2670 & E3.? \\
\hline $\begin{array}{l}\text { AUG } \\
18 \ldots\end{array}$ & 17 & 0 & & & & \\
\hline SEF & & & & & tor & {$[., 16$} \\
\hline $10 \ldots$ & 1650 & 0 & 25.5 & E.08 & 5660 & E1.2 \\
\hline
\end{tabular}


TABLE 12.--Cont inued

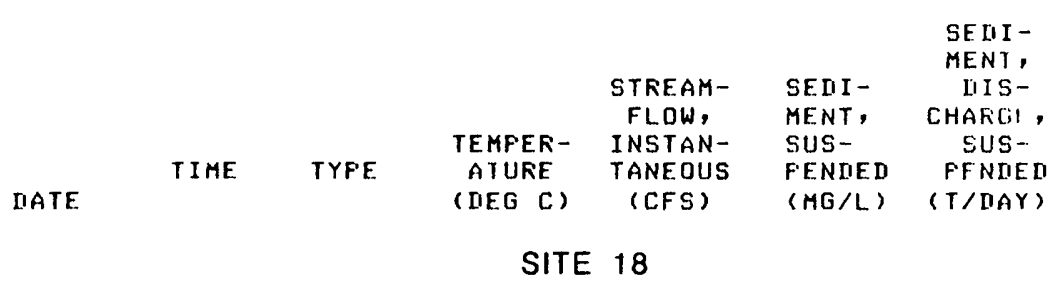

0
0
0
0
0
0
0
0
0
0
0
0
0
0
0
0
0
0
0
0
0
0
0
0
0
0
0
0

$$
17 .
$$

22.4 22.4

21.4

$-$

$-$

$-$

16.2

1.7 .2

18.8

E. 10

.34

152

.14

19.4

16.0

E. 10

1.31

1780

595

1.5

.30

.37

197

.01

2.2

21.0

18.8

19.6

.16

.28

15.0

.14

.16

15.0
14.0

.16
.02

12.6

.29

15.0

.08

11.0

.19

15.0

E. 30

18.4

E. 40

20.9

E. 24

$19 \cdot 3$

E. 80

17.9

E. 08
191

29

20

. 16

.19
.01

$.00 \mathrm{~A}$

$.00 \mathrm{~A}$

E. 02

E.01

E. 26

.01

$18 \quad .01$

1

$$
\begin{array}{ll}
11 & .00 \mathrm{~A} \\
12 & .00 \mathrm{~A}
\end{array}
$$$$
1.00 \mathrm{~A}
$$$$
1.00 \mathrm{~A}
$$

$2.00 A$

$\begin{array}{ll}6 & .00 \mathrm{~A} \\ 4 & .00 \mathrm{~A}\end{array}$

$5.00 \mathrm{~A}$

9 E.O1

51 E.ก6

628 E.41

17 E.ก4

4 E.01




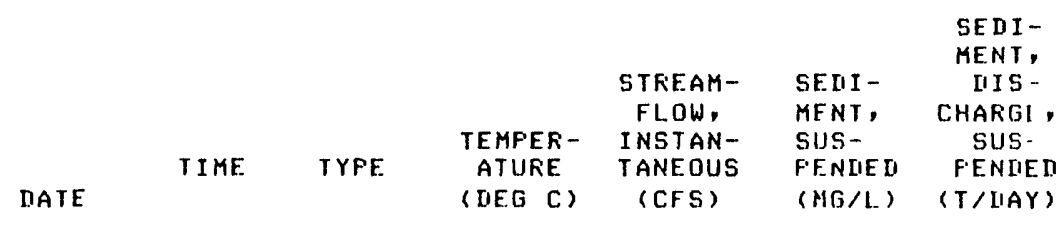

SITE 19

462115119563301 - LNAIN 60.7 AF SEII POND NF SUNNYSIIIE, WA (LAT 462115 LONG 1195633 )

\begin{tabular}{|c|c|c|c|c|c|c|}
\hline \multicolumn{7}{|c|}{$\begin{array}{l}\text { JUN, } \\
02 \ldots\end{array}$} \\
\hline $14 \ldots$ & 1410 & 0 & -- & 1.3 & 6220 & 22 \\
\hline \multicolumn{7}{|l|}{ JUL } \\
\hline $05 \ldots$ & 1045 & 0 & 20.6 & .56 & 2730 & 4.1 \\
\hline \multicolumn{7}{|l|}{ AUG } \\
\hline $01 \ldots$ & 0830 & 0 & 18.2 & 1.1 & 1640 & 4.9 \\
\hline $16 \ldots$ & 1435 & 0 & 24.6 & 1.0 & $9 a 8$ & 2.7 \\
\hline $30 \ldots$ & 1045 & 0 & $19 \cdot 4$ & 1.3 & 804 & 2.8 \\
\hline \multicolumn{7}{|l|}{ SEF } \\
\hline $\begin{array}{l}11 \ldots \\
28 \ldots\end{array}$ & $\begin{array}{l}1150 \\
1245\end{array}$ & $\begin{array}{l}0 \\
0\end{array}$ & $\begin{array}{l}13.7 \\
13.7\end{array}$ & .07 & $\begin{array}{r}19 \\
4\end{array}$ & $\begin{array}{l}.00 \mathrm{~A} \\
.00 \mathrm{~A}\end{array}$ \\
\hline \multicolumn{7}{|l|}{ OCT } \\
\hline $18 \ldots$ & 1300 & 0 & -- & .07 & 256 & .05 \\
\hline \multicolumn{7}{|l|}{ NOU } \\
\hline \multicolumn{5}{|l|}{ IIEC } & 51 & E.01 \\
\hline \multicolumn{6}{|c|}{ FEF , 1980} & E.0OA \\
\hline $20 \ldots$ & 1746 & 0 & $-\cdot \cdot$ & -- & 8060 & -- \\
\hline $21 \ldots$ & 1430 & 0 & - & .10 & 30 & $.00 \mathrm{~A}$ \\
\hline \multicolumn{7}{|l|}{ MAK } \\
\hline \multicolumn{7}{|l|}{ AFF } \\
\hline \multicolumn{6}{|l|}{ MAY } & E.0OA \\
\hline $07 \ldots$ & 1325 & 0 & 20.5 & .64 & 585 & 1.0 \\
\hline $28 \ldots$ & 1350 & 0 & 21.0 & .91 & 157 & .39 \\
\hline \multicolumn{7}{|l|}{ JUN } \\
\hline $11 \ldots$ & 1140 & 0 & 17.0 & E1.1 & 74 & E.22 \\
\hline $25 \ldots$ & 1440 & 0 & $19 \cdot 2$ & .71 & 364 & .70 \\
\hline \multicolumn{7}{|l|}{ JUL } \\
\hline $02 \ldots$ & 1230 & 0 & 22,2 & 1.1 & 2410 & 7.2 \\
\hline $15 \ldots$ & 1400 & 0 & 21.8 & E 1.2 & 1840 & E6.0 \\
\hline $23 \ldots$ & 1147 & 0 & 22.4 & .37 & 444 & .44 \\
\hline \multicolumn{7}{|l|}{ AUG } \\
\hline $08 \ldots$ & 1730 & 0 & 21.0 & .23 & 275 & .17 \\
\hline $21 \ldots$ & 1450 & 0 & 19.4 & .15 & 121 & .05 \\
\hline \multicolumn{7}{|l|}{ SEF } \\
\hline $03 \ldots$ & 1540 & 0 & 17.0 & .25 & 44 & .03 \\
\hline $17 \ldots$ & 1405 & 0 & $18 \cdot 4$ & .18 & 60 & .03 \\
\hline \multicolumn{7}{|l|}{$\mathrm{OCT}$} \\
\hline $09 \ldots$ & 1645 & 0 & 14.2 & .74 & 50 & .10 \\
\hline \multicolumn{7}{|c|}{$A F K, 1981$} \\
\hline \multicolumn{5}{|l|}{ MAY } & 80 & .06 \\
\hline $21 \ldots$ & 1440 & 0 & $22 \cdot 4$ & .72 & 297 & .58 \\
\hline \multicolumn{7}{|l|}{ JUL } \\
\hline $10 \ldots$ & 1440 & 0 & 22.0 & E. 50 & 4560 & E6.2 \\
\hline \multicolumn{5}{|l|}{ AUG } & 1880 & \\
\hline $18 \ldots$ & 0930 & 0 & 18.8 & E1.0 & 300 & E.81 \\
\hline \multicolumn{7}{|l|}{ SEP } \\
\hline $10 \ldots$ & 1600 & 0 & 21.8 & .92 & 283 & $.7(2)$ \\
\hline
\end{tabular}


TABLE 12.--Continued

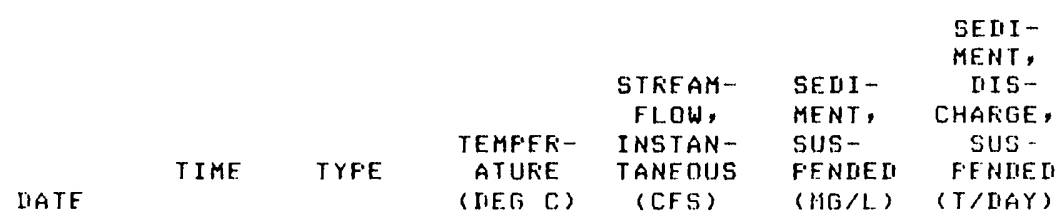

\section{SITE 20}

462115119563302 - IIFAIN 60.7 TRIB AB SEII FOND NR SUNNYSIIIE, WA (LAT $4621 \quad 15$ LONG 1195633 )

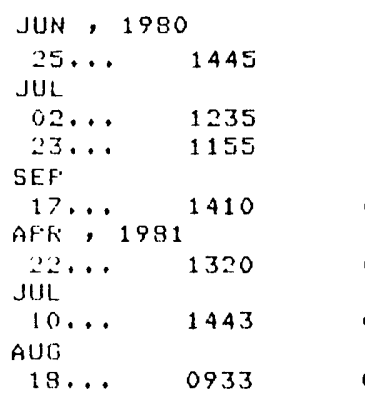

0
0
0
0
0
0
0

16.4

18.7

17.8

.31

.18

E. 04

18.4

E. 02

15.0

.50

26.0

E. 10

20.5

E. 50

SITE 24

462202119552401 - IIRAIN 61.0 ABDUE ROZA CANAL NF SUNNYSIIIE, WA

$$
\begin{array}{ll}
\text { FEB , } & 1980 \\
19 \ldots & 1710 \\
20 \ldots . & 0900 \\
20 \ldots . & 1805
\end{array}
$$

0
0
0

$$
\stackrel{.2}{--}
$$$$
3.8
$$$$
\text { E. } 50
$$$$
2330
$$$$
131
$$$$
\text { E6. } 0
$$

6060

\begin{tabular}{|c|c|c|c|c|c|c|}
\hline $19 \ldots$ & 1725 & 0 & 1 & .43 & 965 & 1.1 \\
\hline $20 \ldots$ & 1815 & 0 & $\cdots$ & E. 80 & 2540 & E5.5 \\
\hline
\end{tabular}

SITE 23

462205119554201 - LIRAIN 60.7 ABOUE ROZA CANAL NR SUNNYSIDE, WA (LAT $4622 \quad 05$ LONG 1195542 ) 
TABLE 12.--Continued

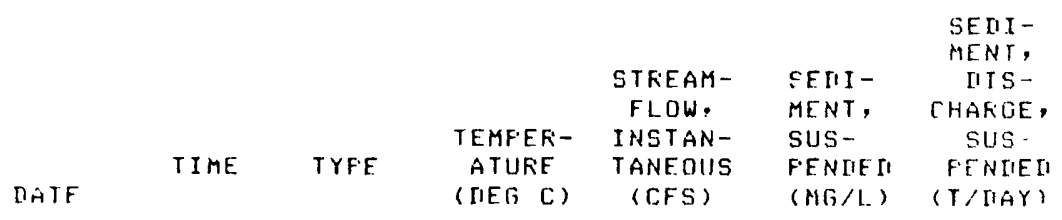

\section{SITE 21}

462114119563501 - IIRAIN 60.7 BL SED FOND NF SUNNYSIDE, WA (LAT 462114 LONG 1195635 )

\begin{tabular}{|c|c|c|c|c|c|c|}
\hline $02 \ldots$ & 1310 & 0 & 22.0 & 1.2 & 2350 & 7.6 \\
\hline $14 \ldots$ & 1415 & 0 & -- & .71 & 713 & 1.4 \\
\hline $\begin{array}{l}25 \ldots \\
\text { JUL. }\end{array}$ & 1510 & 0 & 17.0 & .44 & 249 & .30 \\
\hline $05 \ldots$ & 1140 & 0 & 19.0 & .37 & 324 & .32 \\
\hline $18 \ldots$ & 1020 & 0 & 21.2 & .80 & 371 & .80 \\
\hline \multicolumn{7}{|l|}{ AUG } \\
\hline $01 \ldots$ & 0840 & 0 & 18.4 & .81 & 168 & .37 \\
\hline $16 \ldots$ & 1515 & 0 & $24 \cdot 0$ & .60 & 158 & .26 \\
\hline $30 \ldots$ & 1105 & 0 & 19.6 & .69 & 129 & .24 \\
\hline \multicolumn{7}{|l|}{ SEF } \\
\hline $\begin{array}{l}11 \ldots \\
28 \ldots\end{array}$ & 1200 & 0 & 15.4 & .07 & 11 & $.00 A$ \\
\hline \multicolumn{7}{|l|}{ ocr } \\
\hline $18 \ldots$ & 1305 & 0 & -- & .07 & 100 & .02 \\
\hline $24 \ldots$ & 1345 & 0 & -- & $E+10$ & 10 & E.OOA \\
\hline \multicolumn{7}{|l|}{ NOY } \\
\hline \multicolumn{7}{|l|}{ DEC } \\
\hline $13 \ldots$ & 1300 & 0 & 8.4 & E. 10 & 14 & E.OOA \\
\hline \multicolumn{7}{|c|}{$\mathrm{FEB}, 1980$} \\
\hline $20 \ldots$ & 1745 & 0 & -- & -- & 6760 & -- \\
\hline $21 \ldots$ & 1435 & 0 & -- & E. 10 & 149 & E.04 \\
\hline \multicolumn{7}{|l|}{ MAR } \\
\hline $18 \ldots$ & 1535 & 0 & 9.4 & E. 02 & 33 & $.00 \mathrm{~A}$ \\
\hline \multicolumn{7}{|l|}{ AFK } \\
\hline MAY $23 \cdots$ & \multicolumn{2}{|c|}{ MAY } & 15.0 & .01 & 19 & $.00 \mathrm{~A}$ \\
\hline $07 \ldots$ & 1330 & 0 & 19.4 & .04 & 277 & .03 \\
\hline $28 \ldots$ & 1345 & 0 & 19.0 & .28 & 101 & .08 \\
\hline \multicolumn{7}{|l|}{ JUN } \\
\hline $\begin{array}{l}11 \ldots \\
25 \ldots\end{array}$ & $\begin{array}{l}1145 \\
1450\end{array}$ & $\begin{array}{l}0 \\
0\end{array}$ & $\begin{array}{l}16.6 \\
18.2\end{array}$ & $\begin{array}{l}E .90 \\
1.0\end{array}$ & $\begin{array}{l}61 \\
81\end{array}$ & $\begin{array}{l}.15 \\
.22\end{array}$ \\
\hline \multicolumn{7}{|l|}{ JUL } \\
\hline $02 \ldots$ & 1300 & 0 & 21.0 & E 1.1 & 291 & E.86 \\
\hline $\begin{array}{l}15 \ldots \\
23 \ldots\end{array}$ & $\begin{array}{l}1415 \\
1145\end{array}$ & $\begin{array}{l}0 \\
0\end{array}$ & $\begin{array}{l}22.2 \\
22.0\end{array}$ & $\begin{array}{r}.84 \\
E .41\end{array}$ & $\begin{array}{r}76 \\
126\end{array}$ & $\begin{array}{r}.17 \\
\text { E. } 14\end{array}$ \\
\hline \multicolumn{7}{|l|}{ AUS } \\
\hline $08 \ldots$ & 1755 & 0 & 23.0 & E. 20 & 44 & E.02 \\
\hline \multicolumn{7}{|l|}{ SEF } \\
\hline $\begin{array}{l}03 \ldots \\
17 \ldots\end{array}$ & 1525 & 0 & $17 \cdot 2$ & E.02 & 5 & E.OOA \\
\hline \multicolumn{7}{|l|}{$\mathrm{DCr}$} \\
\hline $09 \ldots$ & 1650 & 0 & 13.8 & E. 10 & 17 & E.OOA \\
\hline \multicolumn{7}{|c|}{ FFF, 1981} \\
\hline \multicolumn{7}{|l|}{ JUL } \\
\hline $10 \ldots$ & 1450 & 0 & 28.0 & E.03 & 360 & E.03 \\
\hline $29 \ldots$ & 1115 & 0 & 20.0 & $E .60$ & 297 & E. 49 \\
\hline \multicolumn{7}{|l|}{ AUO } \\
\hline $18 \ldots$ & 0940 & 0 & 19.6 & E 1.5 & 1010 & E4.1 \\
\hline $\begin{array}{l}\text { SEF } \\
10 \ldots\end{array}$ & 1615 & 0 & 21.0 & E. 10 & 136 & E.04 \\
\hline
\end{tabular}




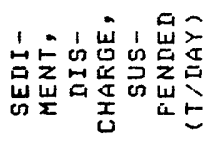

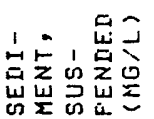

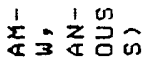

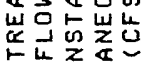

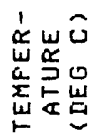

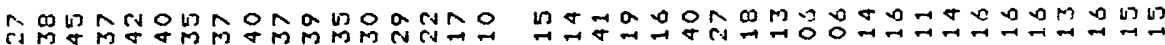

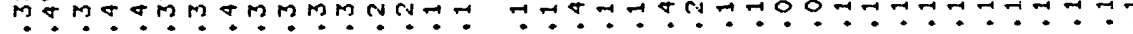

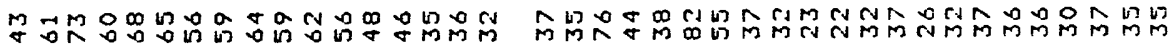

MMMMMMMMMMMMMMMN

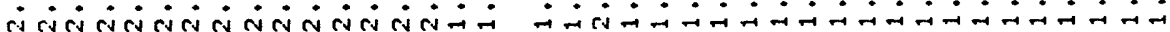

$\underset{2}{2}$

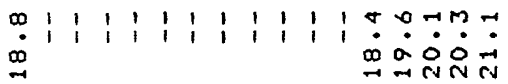

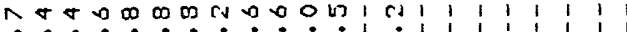

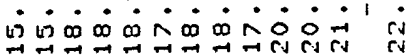

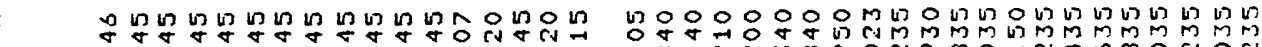

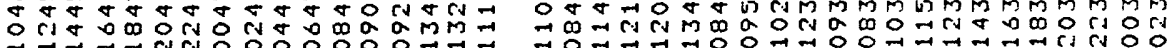

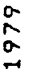

$\underset{\leftarrow}{\stackrel{\omega}{\sigma}}$

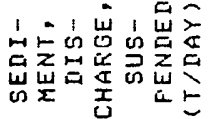

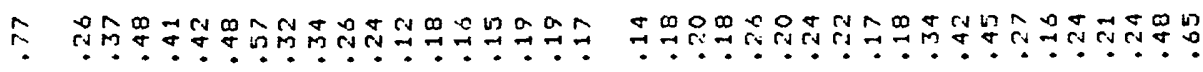

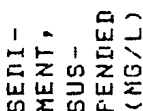

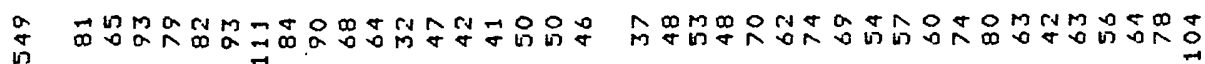

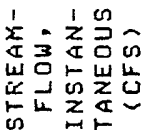

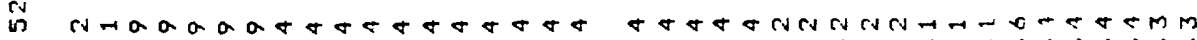

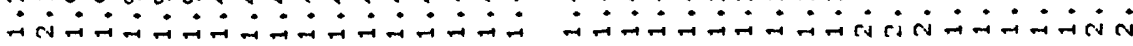

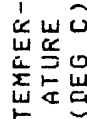

n 0 m

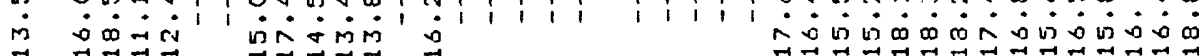

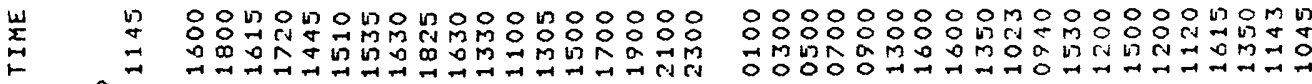
崖

a

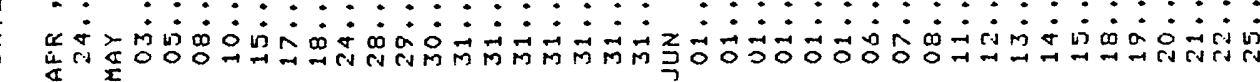


出心出出岕

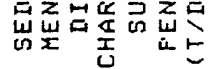

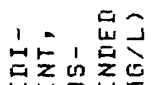

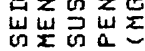

$\frac{1}{2} \approx \frac{1}{2} \stackrel{0}{0}$

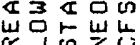

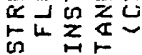

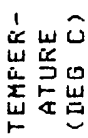

$\underset{⿱ ㇒}{m}$

n

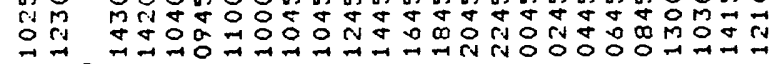

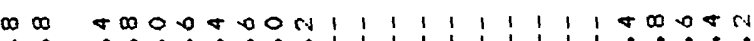

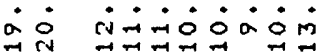

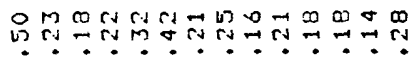

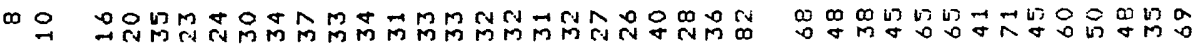

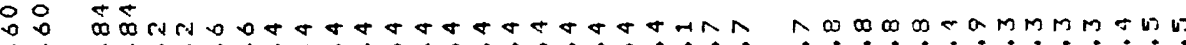

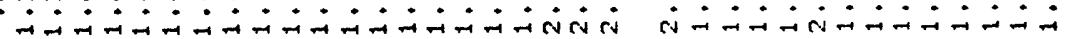

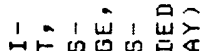

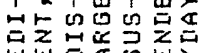

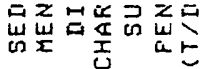

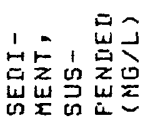

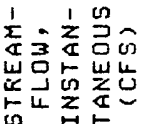

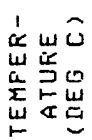

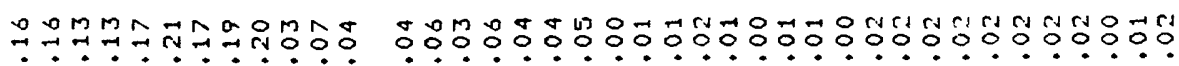

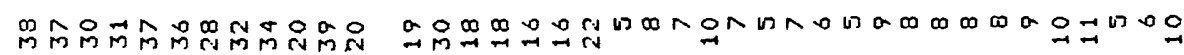

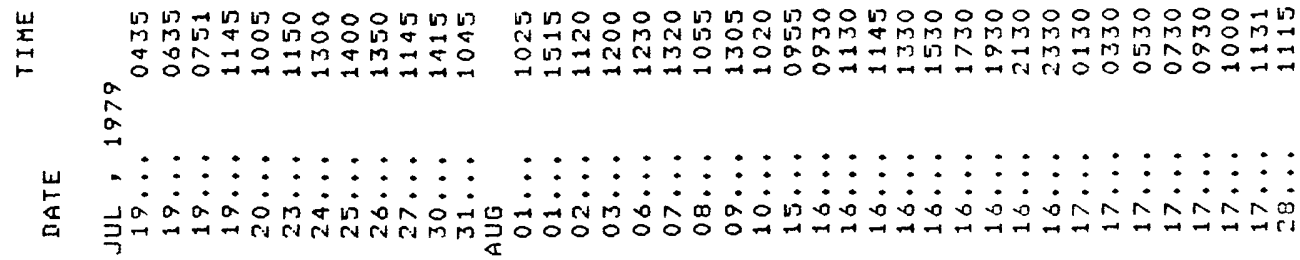




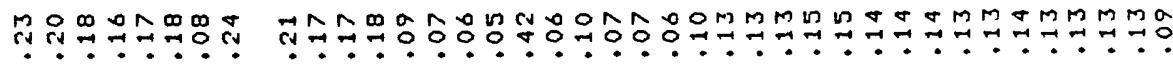

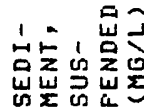

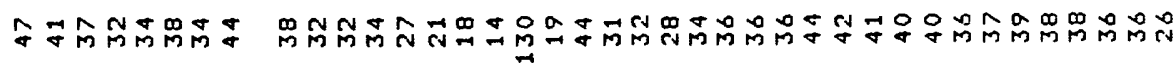

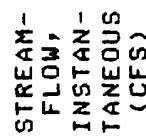
m:

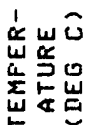

111100

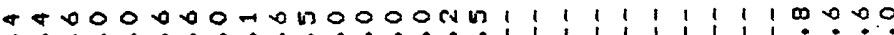

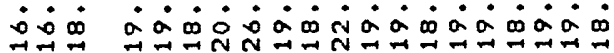

$\therefore \dot{0} \dot{0}$

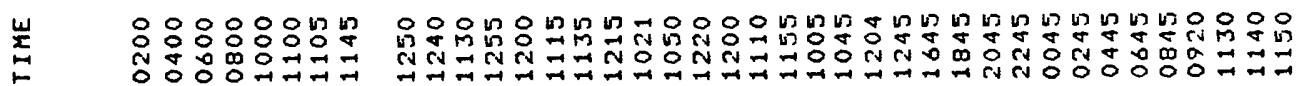
品

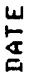

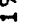

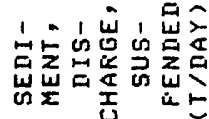

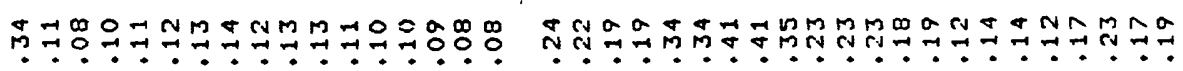

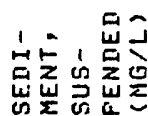

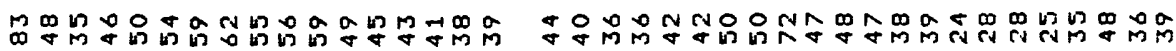

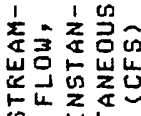

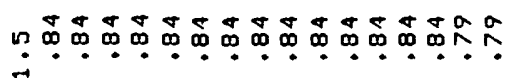

0000000000000000000000

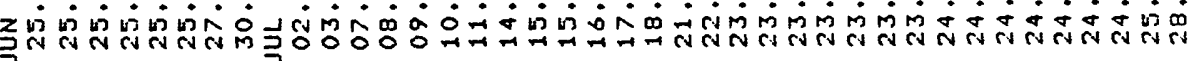

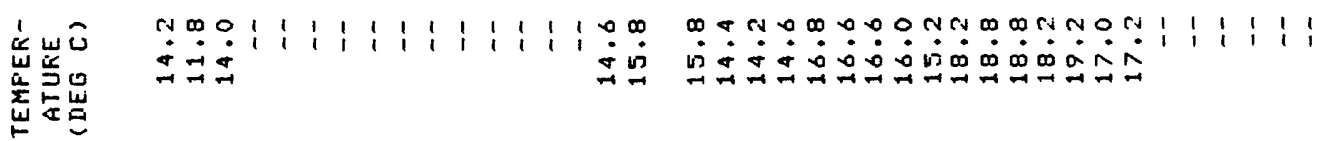

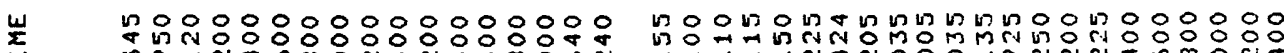

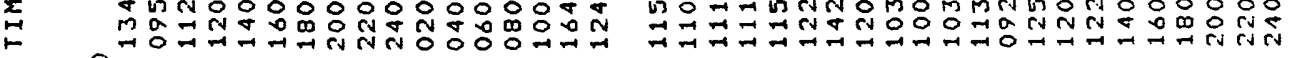
$\stackrel{\infty}{\infty}$

$\underset{\mathfrak{a}}{\mathbf{a}}$

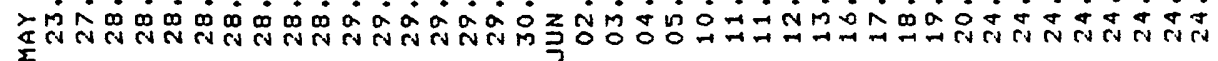




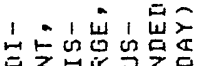

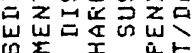

\begin{tabular}{|c|c|c|}
\hline 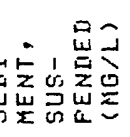 & 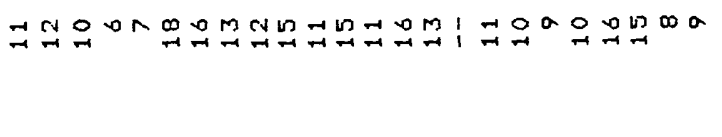 & 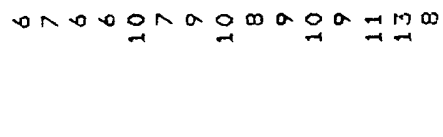 \\
\hline 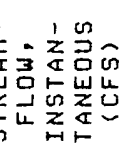 & 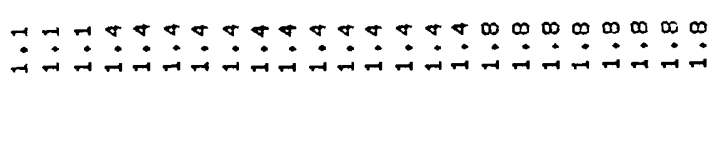 & 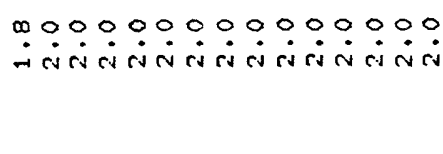 \\
\hline 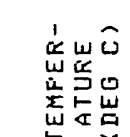 & 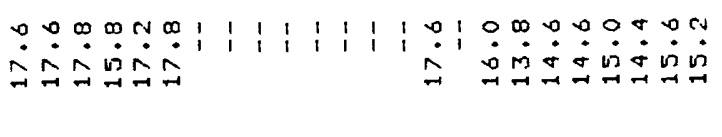 & 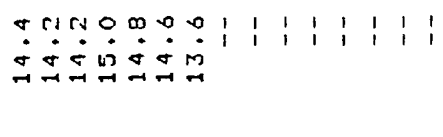 \\
\hline
\end{tabular}

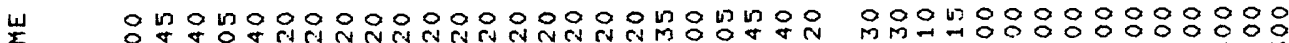
5
5 学

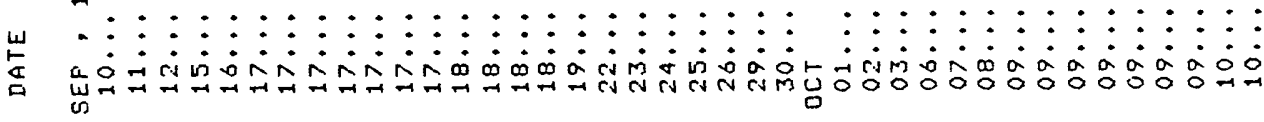

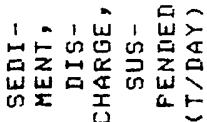

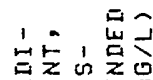

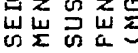

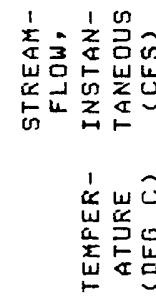

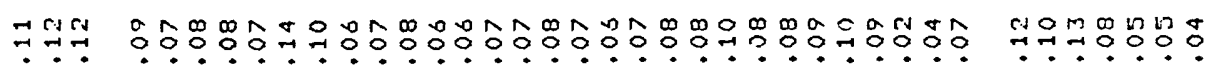

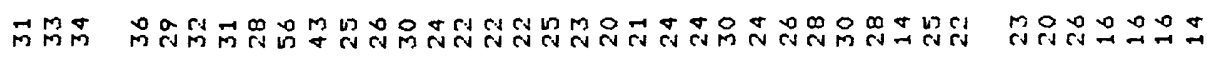

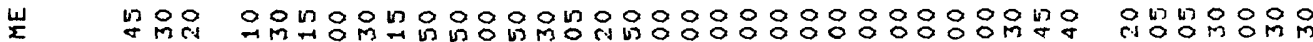

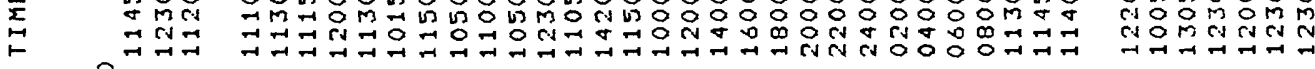
离

000 omatroonomonersoo कि

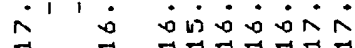

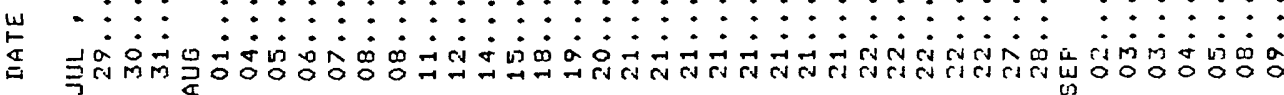




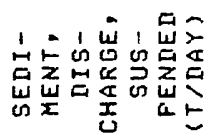

古占崖号

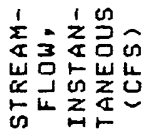

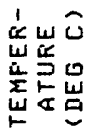

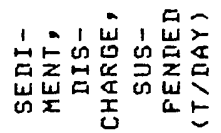

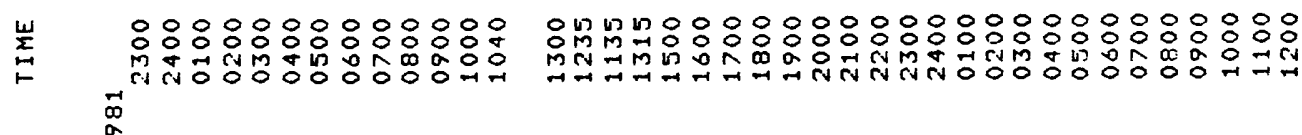
$\underset{a}{\infty}$

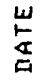
$\frac{1}{4}$ $\frac{1}{x}$

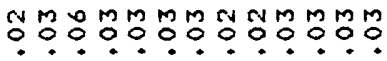

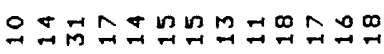

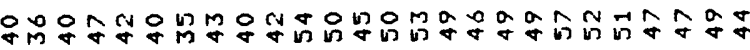

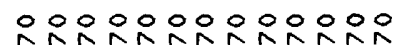

?.?.?.?.?.?.?

D苾

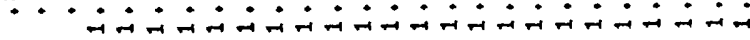

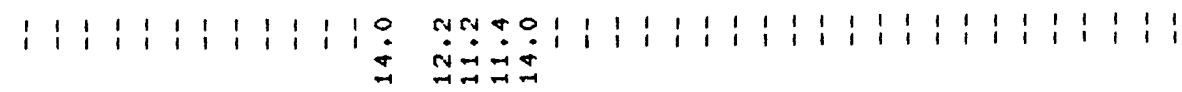

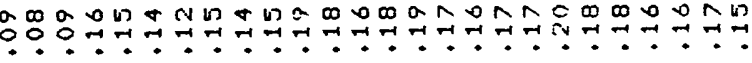

us $0 \dot{0} \dot{0} \dot{0} \dot{0} \dot{0} \dot{0}$

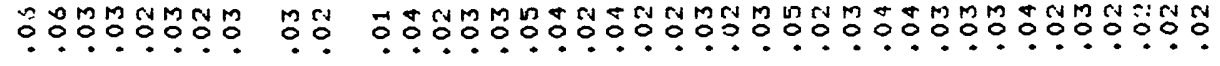

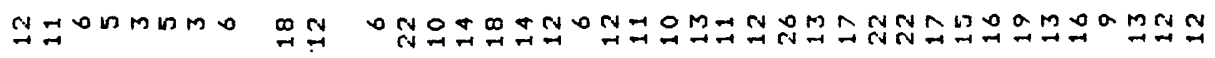

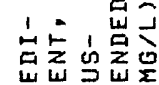
岁崖灵岩要

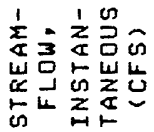

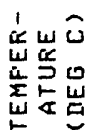

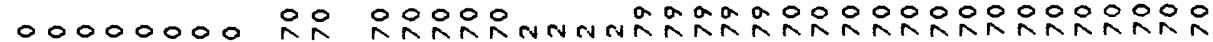

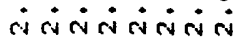
in i- in

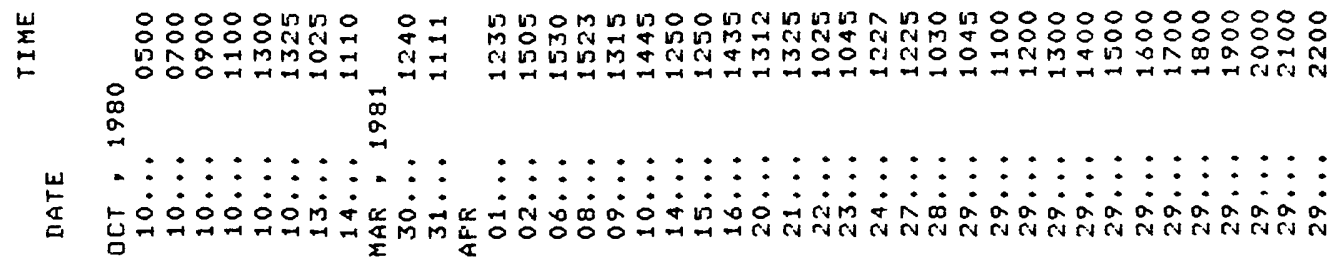




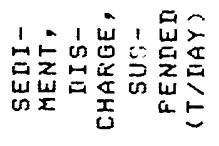

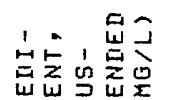

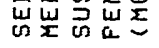

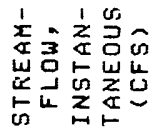

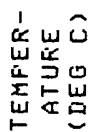

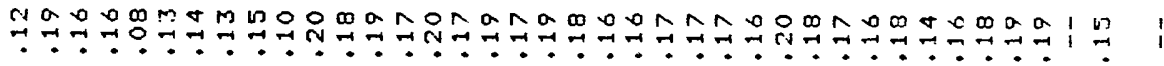

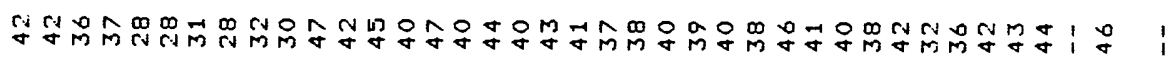

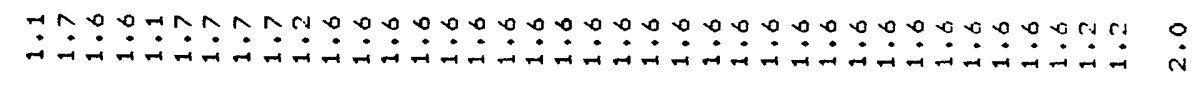

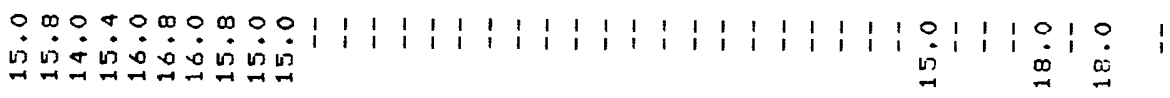

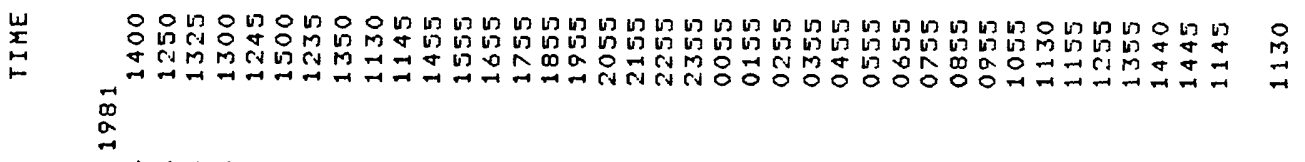

$\stackrel{w}{\leftarrow}$

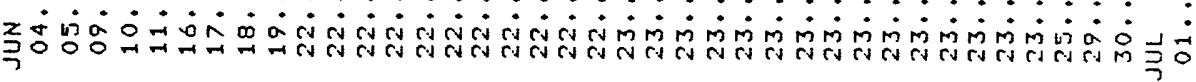

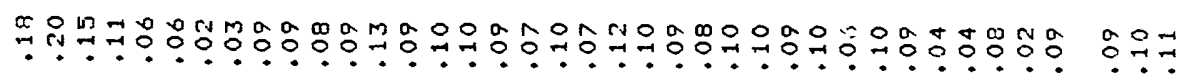

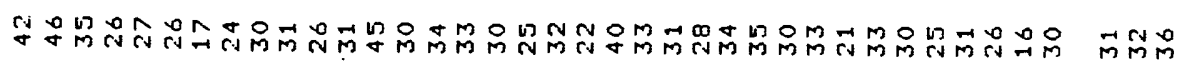

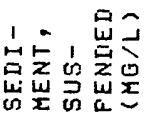

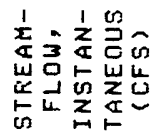

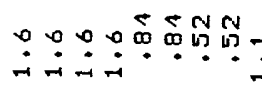

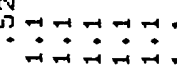

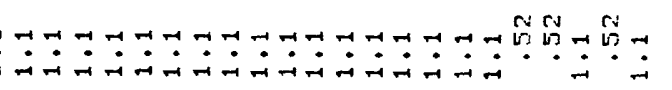

$\because \because \ddot{H}$

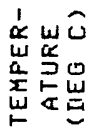

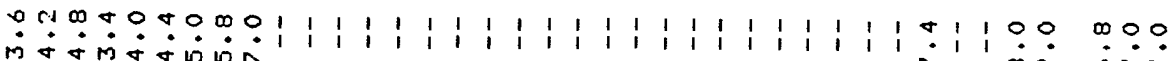

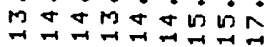

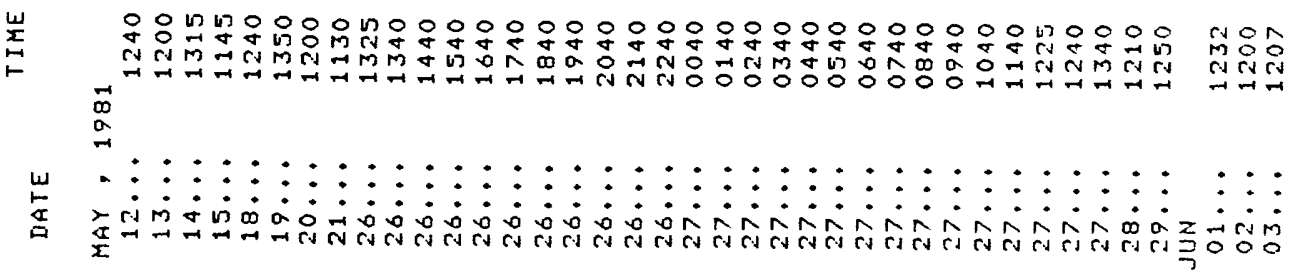




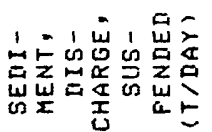

解的崖

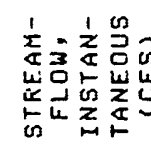

\&ै山

岕岕岕

崖

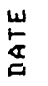

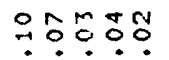

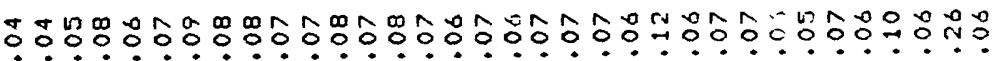

mand

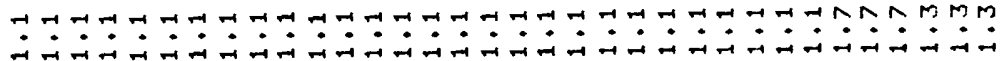

boa 00

$\dot{0}: \dot{0}: \dot{0}$

$\because r \infty !$

$\stackrel{\infty}{\infty} \underset{\sim}{\infty}$

0 nopomon

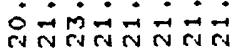

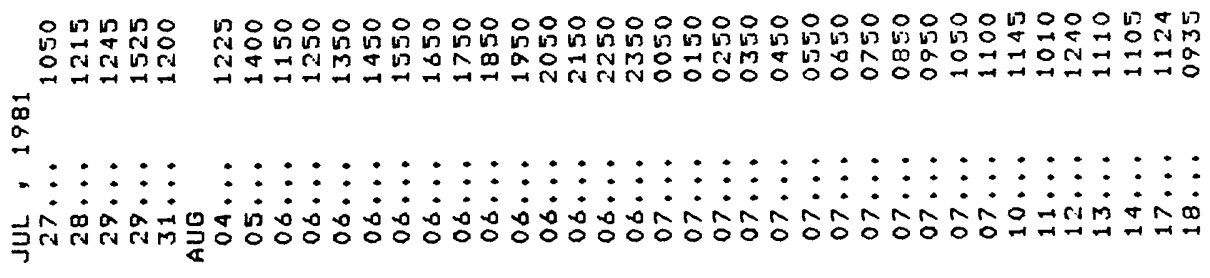

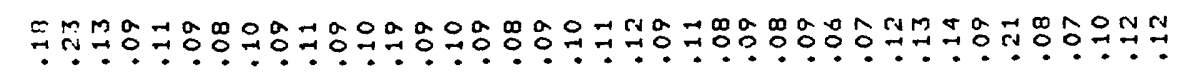

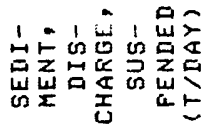

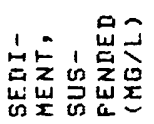

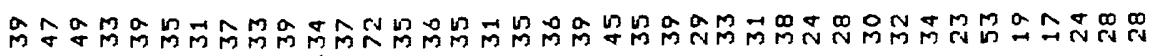

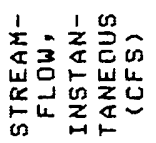

ๆ

¿́⿴囗十丁

采㟧

就崖

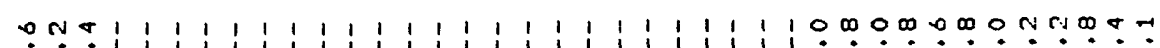
ind

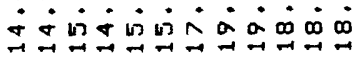

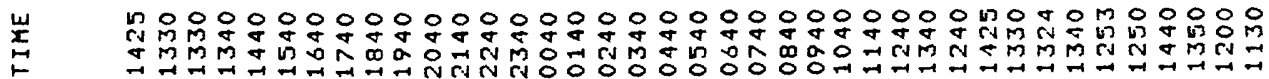
a

压

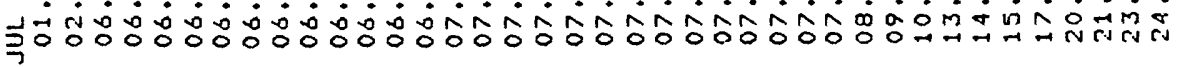




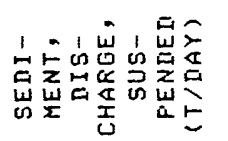

뭉유웅

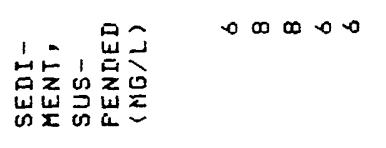

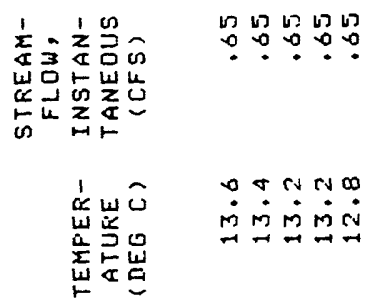

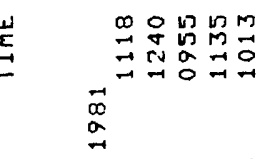

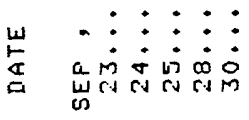

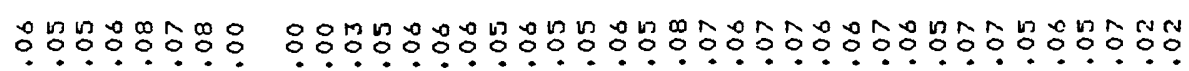

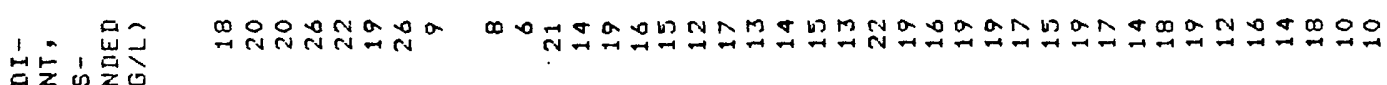

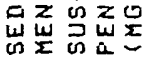

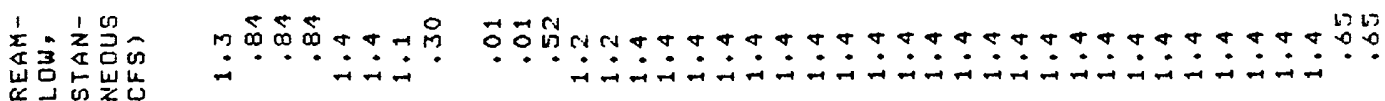

10

00000000

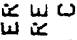

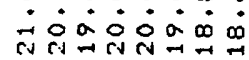

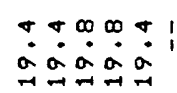

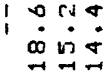

点点岩

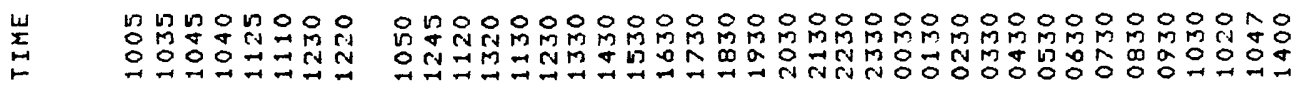
$\stackrel{\overrightarrow{0}}{a}$

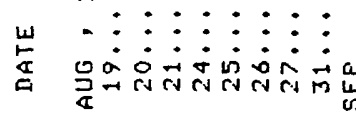

䍃安

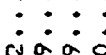

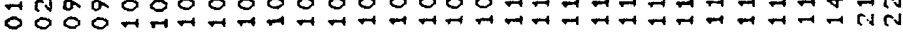




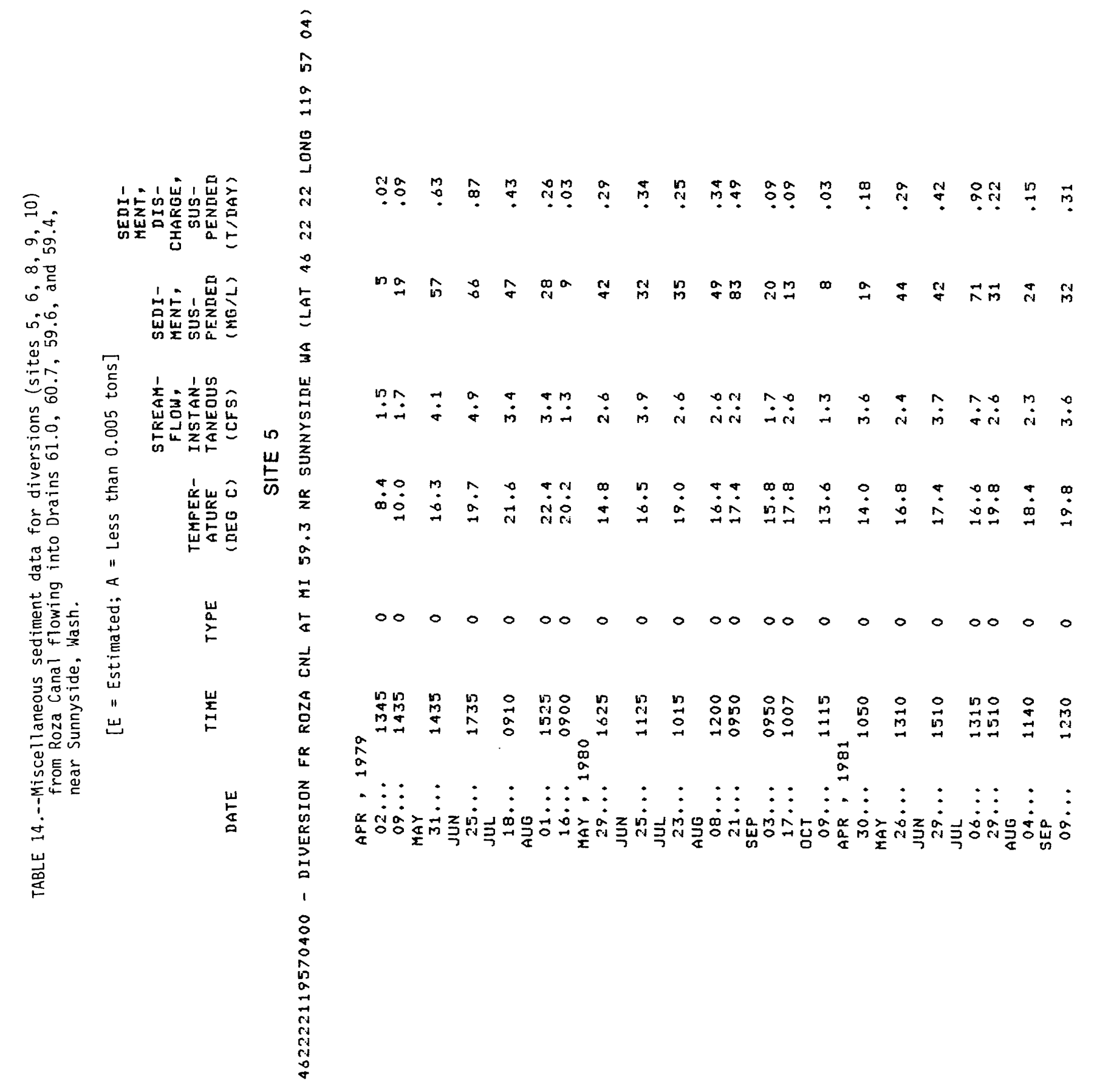




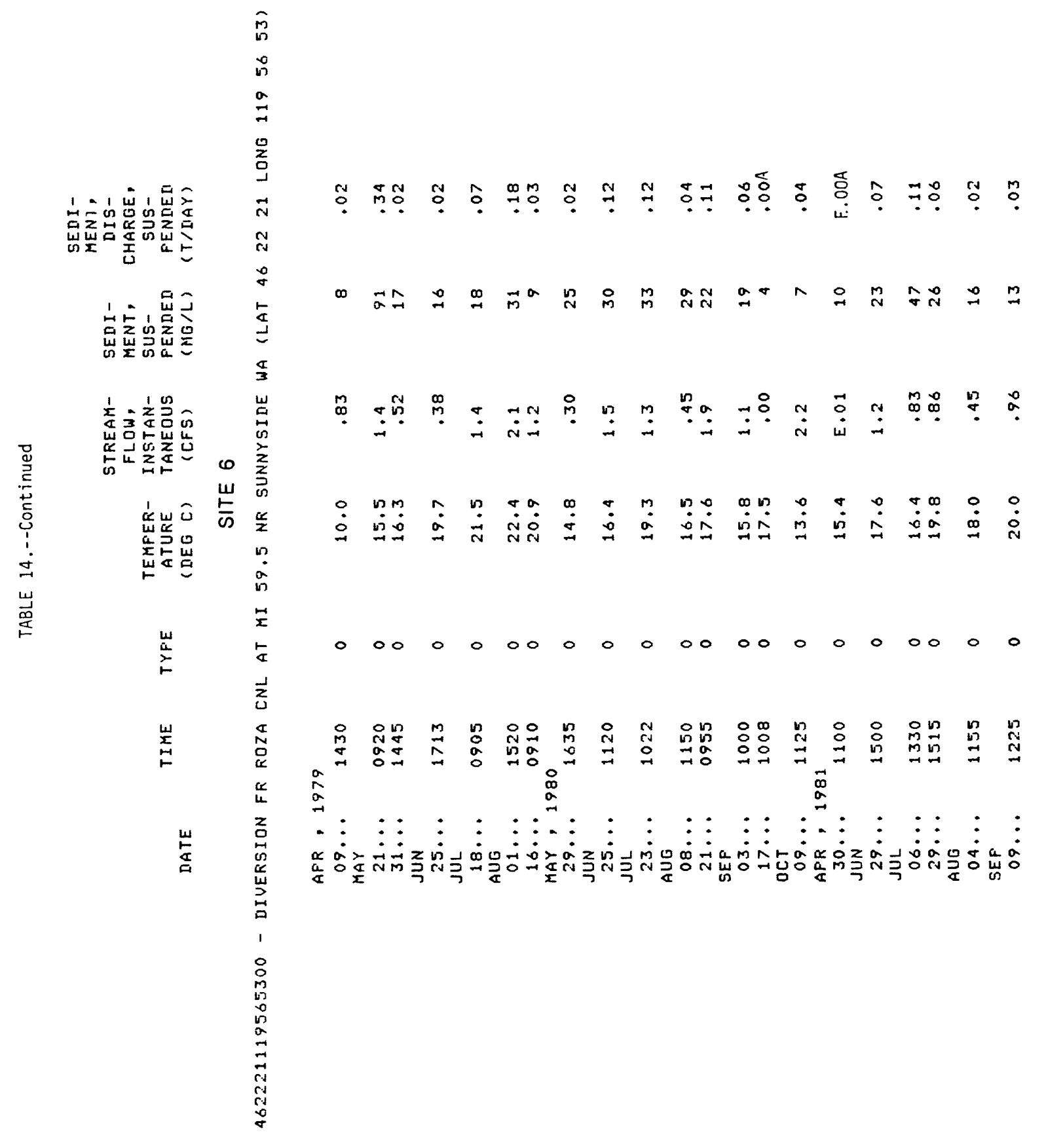




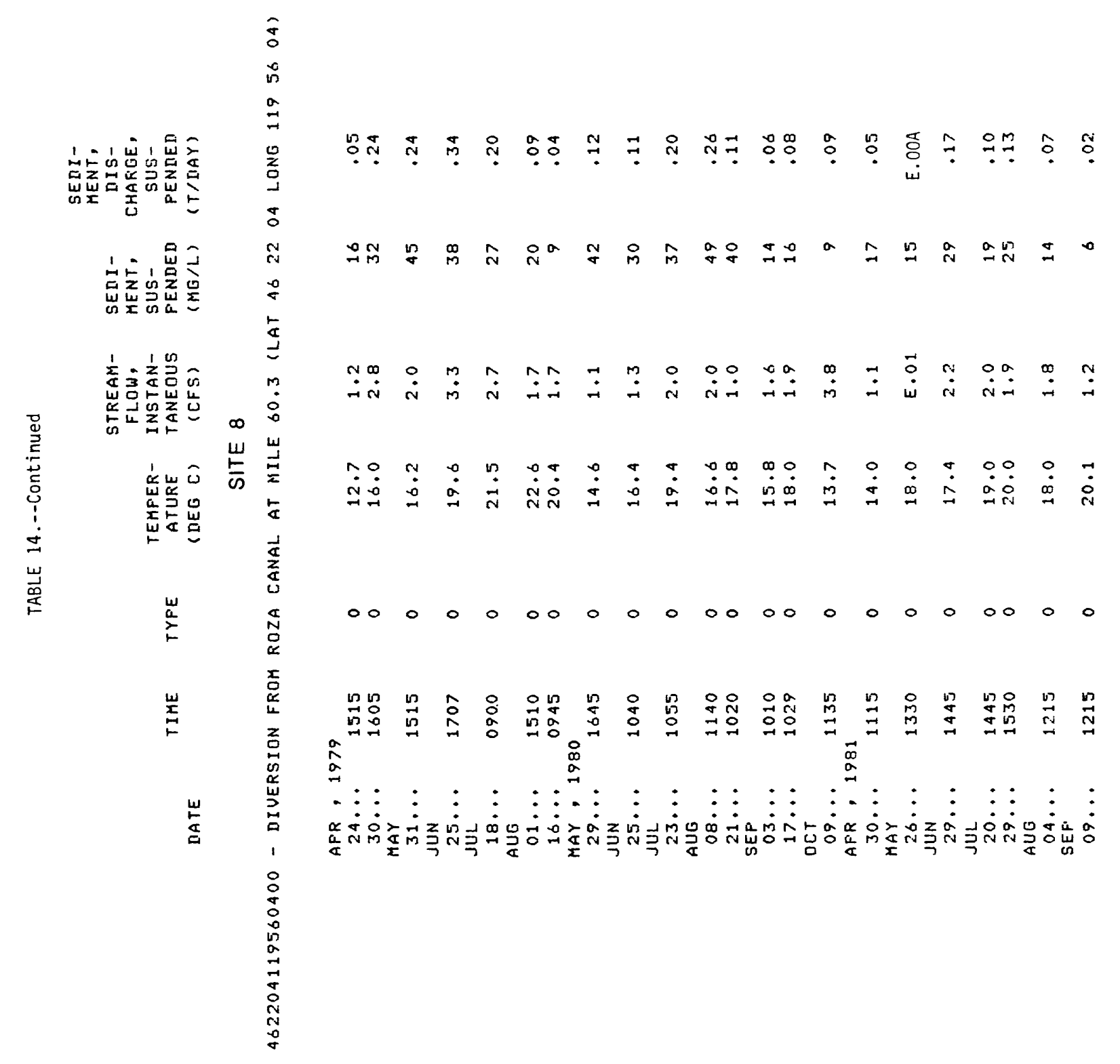




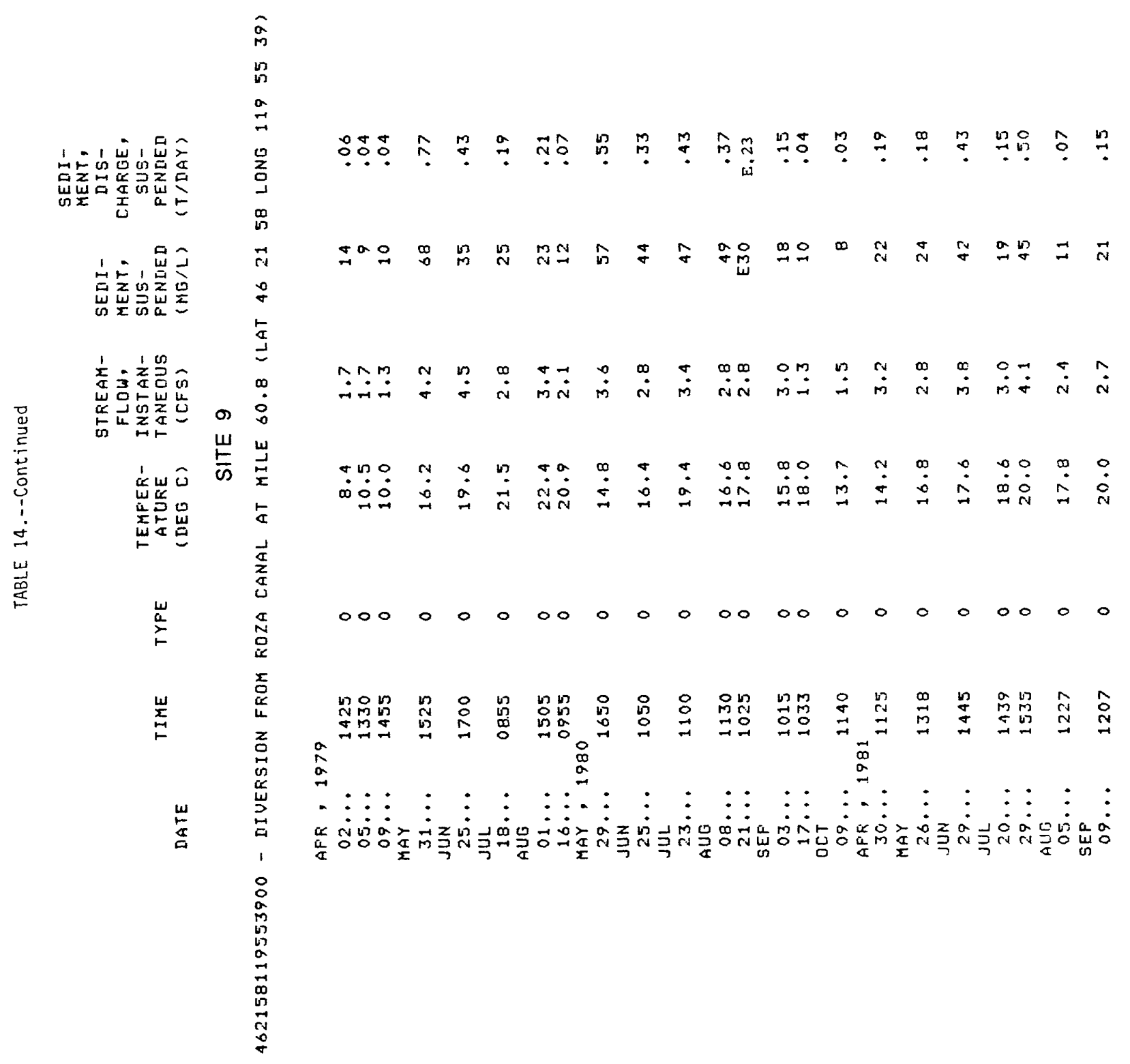




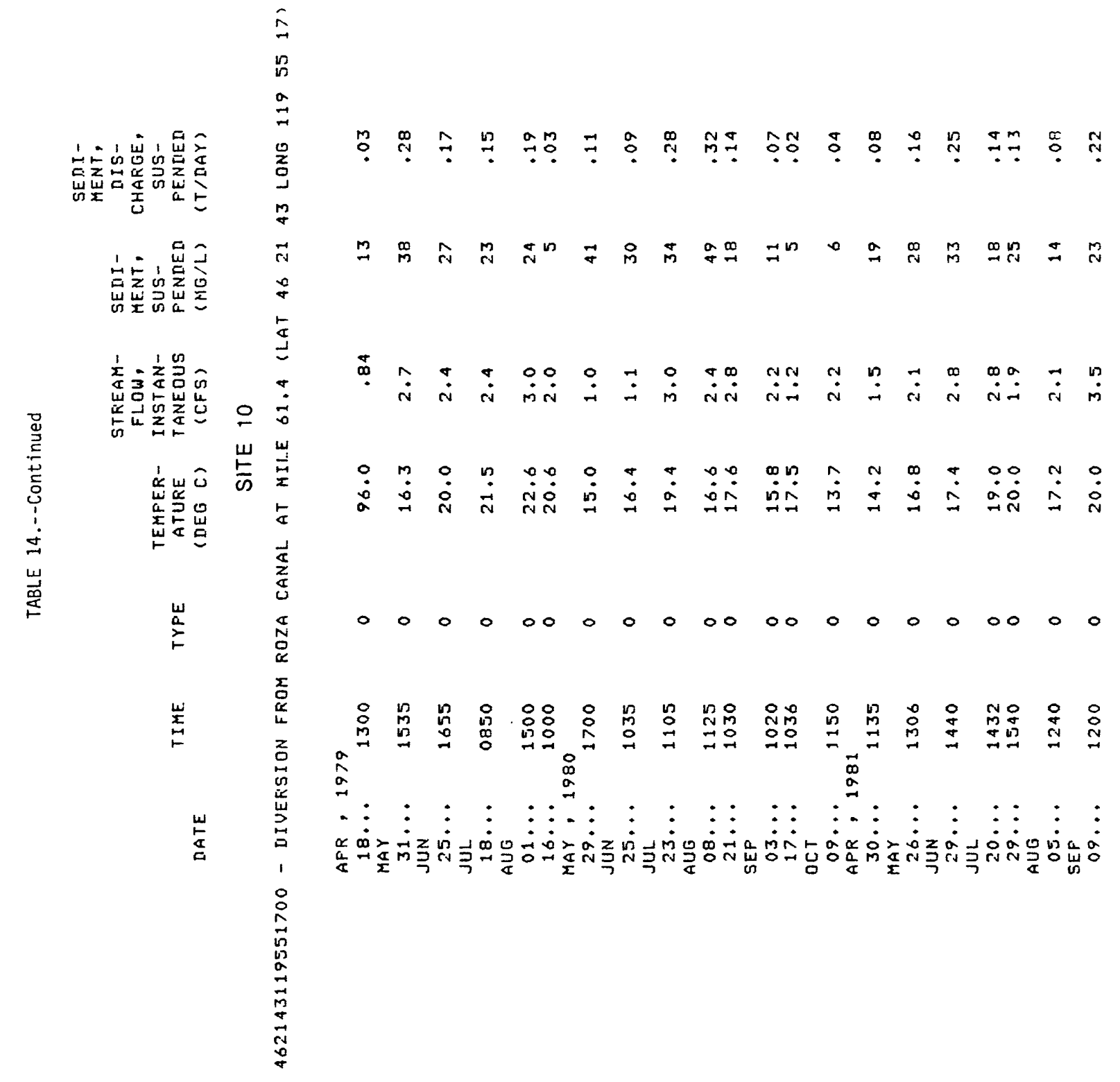

